\title{
Organocatalytic Enantioselective $\gamma$-Elimination: Applications in the Preparation of Chiral Peroxides and Epoxides
}

\author{
Fangli Hu, $\uparrow$ Zhili Chen,$\uparrow$ Yu Tan, $\uparrow$ Da Xu,$\uparrow$ Shengli Huang, $\uparrow$ Shiqi Jia, $\uparrow$ Xiangnan Gong,,+ Wenling Qin* ${ }^{\dagger}$ \\ and Hailong Yan*† \\ †Chongqing Key Laboratory of Natural Product Synthesis and Drug Research, School of Pharmaceutical Sciences, Chongqing \\ University, Chongqing 401331, P. R. China. \\ łAnalytical and Testing Center of Chongqing University, Chongqing University, Chongqing 401331, P. R. China. \\ Corresponding author e-mail: wenling.qin@cqu.edu.cn
}

yhl198151@cqu.edu.cn

\section{Supporting information}

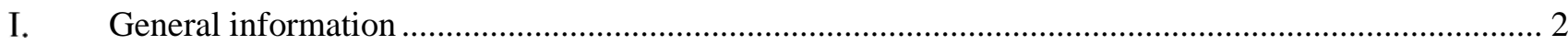

II. General procedure for the synthesis of the substrate …................................................................ 2

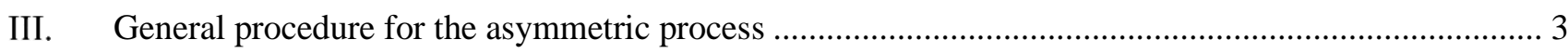

IV. Screening of the reaction conditions and substrate scope …......................................................... 3

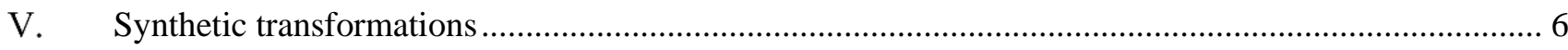

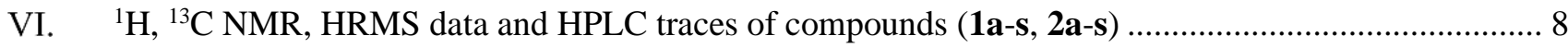

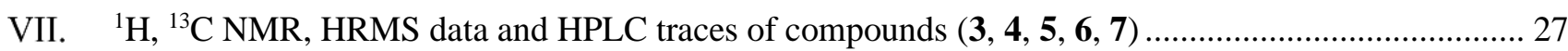

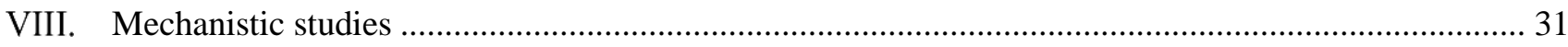

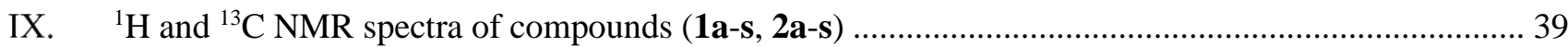

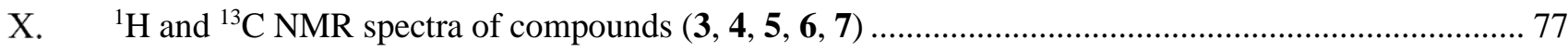

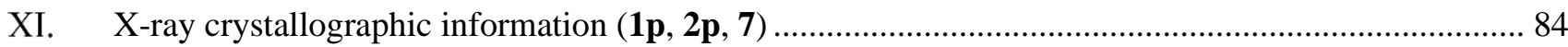

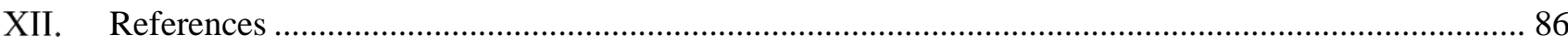




\section{General information}

${ }^{1} \mathrm{H}$ and ${ }^{13} \mathrm{C}$ NMR spectra were recorded on Agilent 400MR DD2 (400 MHz) spectrometer. Chemical shifts were reported in parts per million (ppm), and tetramethylsilane or the residual solvent peak was used as an internal reference: $\mathrm{CDCl}_{3}\left({ }^{1} \mathrm{H}\right.$ NMR tetramethylsilane $\delta 0.00,{ }^{13} \mathrm{C}$ NMR $\delta$ 77.00). Data are reported as follows: chemical shift, multiplicity ( $\mathrm{s}=$ singlet, $\mathrm{d}=$ doublet, $\mathrm{t}=$ triplet, $\mathrm{q}=$ quartet, $\mathrm{m}=$ multiplet, $\mathrm{br}=$ broad $)$, coupling constants $(\mathrm{Hz})$ and integration. Enantiomeric excesses (ee) were determined by HPLC analysis on Hitachi Chromaster using DAICEL CHIRALCEL AD-H, 4.6mm $\Phi \times 250 \mathrm{mmL}$, DAICEL CHIRALCEL OD-H, 4.6mm $\Phi \times 250 \mathrm{mmL}$ and DAICEL CHIRALCEL OJ-H, 4.6mm $\Phi \times 250 \mathrm{mmL}$, DAICEL CHIRALCEL IA-H, 4.6mm $\Phi \times 250 \mathrm{mmL}$. High resolution mass spectra (HRMS) were performed on Bruker Solarix 7.0 T. X-ray crystallography analysis of single crystal was performed on an Agilent SuperNova-CCD X-Ray diffractometer. Optical Rotation was measured on a Rudolph Autopol I polarimeter and are reported as follows: $[\alpha]_{\mathrm{D}}^{20}(c$ in $\mathrm{g}$ per $100 \mathrm{~mL}$ solvent). Melting points were measured using a SGWX-4A microscopy melting point meter and are uncorrected. For reactions that required heating, we used an oil bath to heat. Unless otherwise stated, all reagents were purchased from commercial suppliers (Adamas, J\&K, Sigma-Aldrich, TCI) and used without further purification.

\section{General procedure for the synthesis of the substrate}

Substrates $( \pm)-\mathbf{1}$ were prepared according to the known literature. ${ }^{1}$

Method A: ( \pm )-1a-r<smiles>O=Cc1c[R1]ccc1</smiles>

S1<smiles>[R]=CC=Cc1ccccc1</smiles>

S2

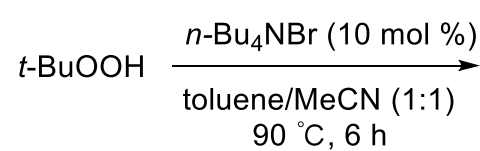

S3<smiles>[R]c1ccccc1C(CC(=O)c1c[R4]ccc1)OOC(C)(C)C</smiles>

$( \pm)-1 a-1 r$<smiles>[R]c1ccccc1C(CC(=O)c1cc[R1]cc1)OOC(C)(C)C</smiles>

Toluene (10 mL) was added to a mixture of $\mathbf{S 1}$ (15 mmol, 3 equiv), $\mathbf{S 2}(5 \mathrm{mmol}, 1$ equiv), and $n-\mathrm{Bu} 4 \mathrm{NBr}$ (161 $\mathrm{mg}, 0.5 \mathrm{mmol}, 0.1$ equiv) under air at room temperature, then $\mathbf{S 3}$ (TBHP, 70\% aq, $2.06 \mathrm{~mL}, 15 \mathrm{mmol}, 3$ equiv) and $\mathrm{MeCN}(10 \mathrm{~mL})$ were added. After the mixture was stirred at $90{ }^{\circ} \mathrm{C}$ for $6 \mathrm{~h}$, the residue was mixed with silica gel and concentrated. The resulting mixture was purified by silica gel column chromatography $\left(\mathrm{SiO}_{2}, \mathrm{PE} / \mathrm{CH}_{2} \mathrm{Cl}_{2}=8: 1\right)$ to give the desired products $( \pm)-\mathbf{1 a}-\mathbf{r}$.

Method B: ( \pm -1s

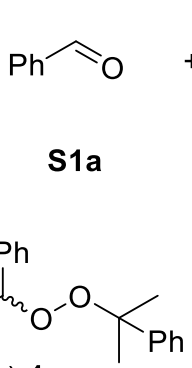

$( \pm)-1 s$<smiles>CC(C)(OOC(CC(=O)c1ccccc1)c1ccccc1)c1ccccc1</smiles>

Toluene $(10 \mathrm{~mL})$ was added to a mixture of benzaldehyde $(1.52 \mathrm{~mL}, 15 \mathrm{mmol}, 3$ equiv), styrene ( $0.57 \mathrm{~mL}, 5 \mathrm{mmol}, 1$ equiv), and $n$ - $\mathrm{Bu} 4 \mathrm{NBr}(161 \mathrm{mg}, 0.5 \mathrm{mmol}, 0.1$ equiv) under air at room temperature, then cumyl hydroperoxide $\mathbf{S 4}$ ( $80 \%$ aq, $2.72 \mathrm{~mL}, 15 \mathrm{mmol}, 3$ equiv) and $\mathrm{MeCN}(10 \mathrm{~mL})$ were added. After the mixture was stirred at $90{ }^{\circ} \mathrm{C}$ for $6 \mathrm{~h}$, the residue 
was mixed with silica gel and concentrated. The resulting mixture was purified by silica gel column chromatography $\left(\mathrm{SiO}_{2}, \mathrm{PE} / \mathrm{CH}_{2} \mathrm{Cl}_{2}=6: 1\right)$ to give the desired product $( \pm)-\mathbf{1 s}$.

\section{General procedure for the asymmetric process}<smiles>[R1]c1cccc(C(=O)C[C@H](OOC([R])(C)C)c2ccccc2[R])c1</smiles>

$( \pm)-1$

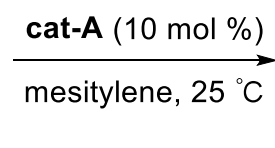

$\mathrm{R}^{1}$<smiles>c1ccccc1</smiles>

(S)-1<smiles>[R4]c1ccc([C@@H]2O[C@H]2C(=O)c2c[R1]ccc2)cc1</smiles>

$(2 R, 3 S)-2$

The substrate $( \pm)-\mathbf{1}(0.1 \mathrm{mmol})$, cat-A $(10 \mathrm{~mol} \%)$ were added to a $10 \mathrm{~mL}$ flame-dried Schlenk tube with a magnetic stirring bar. Mesitylene $(1.5 \mathrm{~mL})$ was injected into the tube. After stirring for specific time, the mixture was purified by flash column chromatography $\left(\mathrm{SiO}_{2}, \mathrm{PE} / \mathrm{CH}_{2} \mathrm{Cl}_{2}=2: 1\right)$ to afford the products and the substrates. Racemic samples were prepared by the same procedure with DBU as catalyst.

\section{Screening of the reaction conditions and substrate scope}<smiles>CC(C)(C)OO[C@H](CC(=O)c1ccccc1)c1ccccc1</smiles>

(土)-1a $\underset{\text { solvent }}{\stackrel{\text { catalyst }}{\longrightarrow}}$

10<smiles>CC(C)(C)OO[C@@H](CC(=O)c1ccccc1)c1ccccc1</smiles>

2a<smiles>C=CC1CN2CCC1C2[C@H](NC(=S)NC1CCCCC1)c1ccnc2ccc(OC)cc12</smiles>

A<smiles>C=CC1CN2CCC1C2[C@H](NC(=O)[C@@H](Nc1c(NC(=O)c2ccccc2)c(=O)c1=O)c1ccnc2ccc(OC)cc12)C(C)C</smiles><smiles>CN(C)[C@@H]1CCCC[C@H]1NC(=S)NC1CCCCC1</smiles>

B<smiles>C=CC1CN2CCC1C2[C@H](NC(=S)NC1CCCCC1)c1ccnc2ccccc12</smiles>

C<smiles>C=CC1CC2CCN1C([C@H](Nc1c(N[C]CCC)c(=O)c1=O)c1ccnc3ccc(OC)cc13)C2</smiles>

D 
Table 1: Catalyst screening ${ }^{a}$

\begin{tabular}{ccccccc}
\hline entry & cat. & $\begin{array}{c}\text { solvent } \\
(0.1 \mathrm{M})\end{array}$ & $\begin{array}{c}\text { ee } \\
\mathbf{1 a}(\%)^{b}\end{array}$ & $\begin{array}{c}\text { ee } \\
\mathbf{2 a}(\%)^{b}\end{array}$ & $\begin{array}{c}\text { conv. } \\
(\%)^{c}\end{array}$ & $s^{d}$ \\
\hline 1 & $\mathbf{A}$ & toluene & 90 & 82 & 52 & 31 \\
2 & $\mathbf{B}$ & toluene & 64 & 87 & 42 & 28 \\
3 & $\mathbf{C}$ & toluene & -32 & -83 & 28 & 15 \\
4 & $\mathbf{D}$ & toluene & 51 & 87 & 37 & 24 \\
5 & $\mathbf{E}$ & toluene & 9 & 39 & 19 & 2 \\
\hline
\end{tabular}

${ }^{a}$ Reaction conditions: $( \pm)-\mathbf{1 a}(0.1 \mathrm{mmol})$ and catalyst $(10 \mathrm{~mol} \%)$ in toluene $(1.0 \mathrm{~mL})$ at $25{ }^{\circ} \mathrm{C}$ for $47 \mathrm{~h} .{ }^{b}$ The ee value was determined by HPLC analysis on a chiral stationary phase. ${ }^{c}$ Conversion $=\left(\mathrm{ee}^{1 \mathrm{la}}\right) /\left(\mathrm{ee}^{\mathrm{la}}+\mathrm{ee}^{2 \mathrm{a}}\right) .{ }^{d}$ The selectivity factors were calculated by the methods of Fiaud: $s=\ln \left[(1-\right.$ conv. $\left.)\left(1-\mathrm{e}^{\mathrm{la}}\right)\right] / \ln \left[(1-\mathrm{conv}).\left(1+\mathrm{ee}^{\mathrm{la}}\right)\right]$.

Table 2: Solvent screening ${ }^{a}$

\begin{tabular}{ccccccc}
\hline entry & cat. & $\begin{array}{c}\text { solvent } \\
(0.1 \mathrm{M})\end{array}$ & $\begin{array}{c}\text { ee } \\
\mathbf{1 a}(\%)^{b}\end{array}$ & $\begin{array}{c}\text { ee } \\
\mathbf{2 a}(\%)^{b}\end{array}$ & $\begin{array}{c}\text { conv. } \\
(\%)^{c}\end{array}$ & $s^{d}$ \\
\hline 1 & $\mathbf{A}$ & toluene & 90 & 82 & 52 & 31 \\
2 & $\mathbf{A}$ & $m$-xylene & 75 & 86 & 47 & 30 \\
3 & $\mathbf{A}$ & $p$-xylene & 55 & 90 & 38 & 33 \\
4 & $\mathbf{A}$ & mesitylene & 83 & 86 & 49 & 34 \\
5 & $\mathbf{A}$ & $\mathrm{CH}_{2} \mathrm{Cl}_{2}$ & 50 & 89 & 36 & 28 \\
6 & $\mathbf{A}$ & $\mathrm{CHCl}_{3}$ & 30 & 91 & 25 & 28 \\
7 & $\mathbf{A}$ & ethyl acetate & 32 & 84 & 28 & 16 \\
8 & $\mathbf{A}$ & $\mathrm{THF}$ & 6 & 78 & 7 & 9 \\
9 & $\mathbf{A}$ & acetone & 27 & 80 & 25 & 12 \\
\hline
\end{tabular}

${ }^{a}$ Reaction conditions: $( \pm)-\mathbf{1 a}(0.1 \mathrm{mmol})$ and cat-A $(10 \mathrm{~mol} \%)$ in solvent $(1.0 \mathrm{~mL})$ at $25^{\circ} \mathrm{C}$ for $47 \mathrm{~h} .{ }^{b}$ The ee value was determined by HPLC analysis on a chiral stationary phase. ${ }^{c}$ Conversion $=\left(\mathrm{ee}^{1 \mathrm{a}}\right) /\left(\mathrm{ee}^{\mathrm{ia}}+\mathrm{ee}^{2 \mathrm{a}}\right) .{ }^{d}$ The selectivity factors were calculated by the methods of Fiaud: $s=\ln \left[(1-\right.$ conv. $\left.)\left(1-e^{1 a}\right)\right] / \ln \left[(1-\right.$ conv. $\left.)\left(1+e^{1 a}\right)\right]$.

Table 3: Concentration screening ${ }^{[a]}$

\begin{tabular}{ccccccc}
\hline entry & solvent & concentration & $\begin{array}{c}\text { ee } \\
\mathbf{1 a}(\%)^{b}\end{array}$ & $\begin{array}{c}\text { ee } \\
\mathbf{2 a}(\%)^{b}\end{array}$ & $\begin{array}{c}\text { conv. } \\
(\%)^{c}\end{array}$ & $s^{d}$ \\
\hline 1 & mesitylene & $0.2 \mathrm{M}$ & 96 & 78 & 55 & 31 \\
2 & mesitylene & $0.13 \mathrm{M}$ & 92 & 81 & 53 & 31 \\
3 & mesitylene & $0.1 \mathrm{M}$ & 83 & 86 & 49 & 34 \\
4 & mesitylene & $0.07 \mathrm{M}$ & 94 & 84 & 53 & 40 \\
5 & mesitylene & $0.05 \mathrm{M}$ & 72 & 88 & 45 & 34 \\
\hline
\end{tabular}

${ }^{a}$ Reaction conditions: $( \pm)-\mathbf{1 a}(0.1 \mathrm{mmol})$ and cat-A $(10 \mathrm{~mol} \%)$ in mesitylene at $25{ }^{\circ} \mathrm{C}$ for $47 \mathrm{~h} .{ }^{b}$ The ee value was determined by HPLC analysis on a chiral stationary phase. ${ }^{c}$ Conversion $=\left(e^{1 \mathrm{a}}\right) /\left(\mathrm{ee}^{\mathrm{la}}+\mathrm{ee}^{2 \mathrm{a}}\right) .{ }^{d}$ The selectivity factors were calculated by the methods of Fiaud: $s=\ln \left[(1-\right.$ conv. $\left.)\left(1-\mathrm{ee}^{1 \mathrm{a} a}\right)\right] / \ln \left[(1-\right.$ conv. $\left.)\left(1+\mathrm{ee}^{\mathrm{la}}\right)\right]$.

Table 4: Temperature screening ${ }^{a}$

\begin{tabular}{ccccccc}
\hline entry & solvent & $\begin{array}{c}T \\
\left({ }^{\circ} \mathrm{C}\right)\end{array}$ & $\begin{array}{c}\text { ee } \\
\mathbf{1 a}(\%)^{b}\end{array}$ & $\begin{array}{c}\text { ee } \\
\mathbf{2 a}(\%)^{b}\end{array}$ & $\begin{array}{c}\text { conv. } \\
(\%)^{c}\end{array}$ & $s^{d}$ \\
\hline 1 & mesitylene & 0 & 13 & 85 & 13 & 14 \\
2 & mesitylene & 15 & 63 & 87 & 42 & 27
\end{tabular}




\begin{tabular}{lllllll}
3 & mesitylene & 25 & 94 & 84 & 53 & 40 \\
4 & mesitylene & 35 & 77 & 71 & 52 & 13 \\
\hline
\end{tabular}

${ }^{a}$ Reaction conditions: $( \pm)-\mathbf{1 a}(0.1 \mathrm{mmol})$ and cat-A $(10 \mathrm{~mol} \%)$ in mesitylene $(1.5 \mathrm{~mL})$ at corresponding temperature for $47 \mathrm{~h} .{ }^{b} \mathrm{The}$ ee value was determined by HPLC analysis on a chiral stationary phase ${ }^{c}$ Conversion $=\left(\mathrm{ee}^{1 \mathrm{a}}\right) /\left(\mathrm{ee}^{1 \mathrm{a}}+\mathrm{ee}^{2 \mathrm{a}}\right) .{ }^{d}$ The selectivity factors were calculated by the methods of Fiaud: $s=\ln \left[(1-\right.$ conv. $\left.)\left(1-\mathrm{ee}^{\mathrm{la}}\right)\right] / \ln \left[(1-\mathrm{conv}).\left(1+\mathrm{ee}^{\mathrm{la}}\right)\right]$.

Table 5: Substrate scope ${ }^{a}$<smiles>[R]C(=O)CC([R])OOC([R])(C)C</smiles>

$( \pm)-1$<smiles>[R]C(=O)CC([R])OOC([R])(C)C</smiles>

$(S)-1$<smiles>[R]C(=O)[C@@H]1O[C@@H]1[R]</smiles>

$(2 R, 3 S)-2$

\begin{tabular}{|c|c|c|c|c|c|c|c|c|c|c|c|}
\hline \multirow[b]{2}{*}{ entry } & \multirow[b]{2}{*}{$\mathrm{R}$} & \multirow[b]{2}{*}{$\mathrm{R}^{\prime}$} & \multirow[b]{2}{*}{$\mathrm{R}^{3}$} & \multirow[b]{2}{*}{$\begin{array}{r}\text { time } \\
(\mathrm{h})\end{array}$} & \multirow[b]{2}{*}{$\begin{array}{c}\text { conv. } \\
(\%)\end{array}$} & \multicolumn{2}{|c|}{1} & \multirow[b]{2}{*}{$\begin{array}{c}\text { yield } \\
(\%)\end{array}$} & \multirow{2}{*}{$\begin{array}{c}2 \\
\text { ee } \\
(\%)\end{array}$} & \multirow[b]{2}{*}{$\mathrm{dr}$} & \multirow[b]{2}{*}{$s$} \\
\hline & & & & & & $\begin{array}{r}\text { yield } \\
(\%)\end{array}$ & $\begin{array}{c}\text { ee } \\
(\%)\end{array}$ & & & & \\
\hline 1 & $\mathrm{Ph}$ & $\mathrm{Ph}$ & $\mathrm{Me}$ & 47.0 & 53 & 46 & 94 & 49 & 84 & $>20: 1$ & 40 \\
\hline 2 & $4-\mathrm{MeC}_{6} \mathrm{H}_{4}$ & $\mathrm{Ph}$ & $\mathrm{Me}$ & 114.0 & 53 & 45 & 93 & 50 & 83 & $>20: 1$ & 36 \\
\hline 3 & $4-t-\mathrm{BuC}_{6} \mathrm{H}_{4}$ & $\mathrm{Ph}$ & $\mathrm{Me}$ & 92.0 & 56 & 43 & 94 & 52 & 74 & $>20: 1$ & 23 \\
\hline 4 & $3,4-(\mathrm{Me})_{2} \mathrm{C}_{6} \mathrm{H}_{3}$ & $\mathrm{Ph}$ & $\mathrm{Me}$ & 88.0 & 50 & 48 & 87 & 48 & 86 & $>20: 1$ & 38 \\
\hline 5 & $3-\mathrm{OMeC}_{6} \mathrm{H}_{4}$ & $\mathrm{Ph}$ & $\mathrm{Me}$ & 48.0 & 51 & 46 & 86 & 44 & 83 & $>20: 1$ & 30 \\
\hline \multirow{2}{*}{6} & $3,4,5-(\mathrm{MeO})_{3}$ & & & & & & & & & & \\
\hline & $\mathrm{C}_{6} \mathrm{H}_{2}$ & $\mathrm{Ph}$ & $\mathrm{Me}$ & 65.0 & 49 & 47 & 89 & 45 & 91 & $>20: 1$ & 63 \\
\hline 7 & $4-\mathrm{ClC}_{6} \mathrm{H}_{4}$ & $\mathrm{Ph}$ & $\mathrm{Me}$ & 64.0 & 54 & 44 & 90 & 50 & 76 & $>20: 1$ & 22 \\
\hline 8 & $4-\mathrm{BrC}_{6} \mathrm{H}_{4}$ & $\mathrm{Ph}$ & $\mathrm{Me}$ & 65.5 & 55 & 43 & 95 & 52 & 79 & $>20: 1$ & 31 \\
\hline 9 & 2-naphthyl & $\mathrm{Ph}$ & $\mathrm{Me}$ & 74.7 & 51 & 44 & 87 & 48 & 83 & $>20: 1$ & 30 \\
\hline 10 & 2-furanyl & $\mathrm{Ph}$ & $\mathrm{Me}$ & 35.5 & 53 & 45 & 93 & 50 & 83 & $>20: 1$ & 36 \\
\hline 11 & 2-thienyl & $\mathrm{Ph}$ & $\mathrm{Me}$ & 47.0 & 52 & 46 & 93 & 51 & 85 & $>20: 1$ & 42 \\
\hline 12 & $\mathrm{Ph}$ & $4-\mathrm{MeC}_{6} \mathrm{H}_{4}$ & $\mathrm{Me}$ & 112.5 & 55 & 43 & 98 & 51 & 80 & $>20: 1$ & 40 \\
\hline 13 & $\mathrm{Ph}$ & $4-t-\mathrm{BuC}_{6} \mathrm{H}_{4}$ & $\mathrm{Me}$ & 137.0 & 53 & 44 & 93 & 49 & 82 & $>20: 1$ & 34 \\
\hline 14 & $\mathrm{Ph}$ & $4-\mathrm{FC}_{6} \mathrm{H}_{4}$ & $\mathrm{Me}$ & 108.5 & 55 & 43 & 97 & 51 & 80 & $>20: 1$ & 37 \\
\hline 15 & $\mathrm{Ph}$ & $4-\mathrm{ClC}_{6} \mathrm{H}_{4}$ & $\mathrm{Me}$ & 87.5 & 53 & 45 & 91 & 50 & 80 & $>20: 1$ & 28 \\
\hline 16 & $\mathrm{Ph}$ & $4-\mathrm{BrC}_{6} \mathrm{H}_{4}$ & $\mathrm{Me}$ & 73.5 & 52 & 42 & 91 & 51 & 84 & $>20: 1$ & 36 \\
\hline 17 & $\mathrm{Ph}$ & $2-\mathrm{ClC}_{6} \mathrm{H}_{4}$ & $\mathrm{Me}$ & 48.0 & 59 & 40 & 96 & 49 & 67 & $>20: 1$ & 19 \\
\hline 18 & $\mathrm{Ph}$ & $3-\mathrm{BrC}_{6} \mathrm{H}_{4}$ & $\mathrm{Me}$ & 68.5 & 54 & 42 & 96 & 48 & 81 & $>20: 1$ & 37 \\
\hline 19 & $\mathrm{Ph}$ & $\mathrm{Ph}$ & $\mathrm{Ph}$ & 15.0 & 56 & 41 & 87 & 46 & 69 & $>20: 1$ & 15 \\
\hline
\end{tabular}

${ }^{a}$ Reaction conditions: $( \pm)-\mathbf{1}(0.1 \mathrm{mmol})$, cat-A $(10 \mathrm{~mol} \%)$ in mesitylene $(1.5 \mathrm{~mL})$ at $25{ }^{\circ} \mathrm{C}$, stirring for specific time. The ee value was determined by HPLC analysis on a chiral stationary phase. Isolated yield. Conversion $=\left(\mathrm{ee}^{1}\right) /\left(\mathrm{ee}^{1}+\mathrm{ee}^{2}\right)$. Diastereomeric ratio $(\mathrm{dr})$ was determined by ${ }^{1} \mathrm{H}$ NMR analysis of the crude reaction mixture. The selectivity factors were calculated by the methods of Fiaud: $s=\ln [(1$ - conv. $\left.)\left(1-e^{1}\right)\right] / \ln \left[(1-\right.$ conv. $\left.)\left(1+e^{1}\right)\right]$. 


\section{Synthetic transformations}

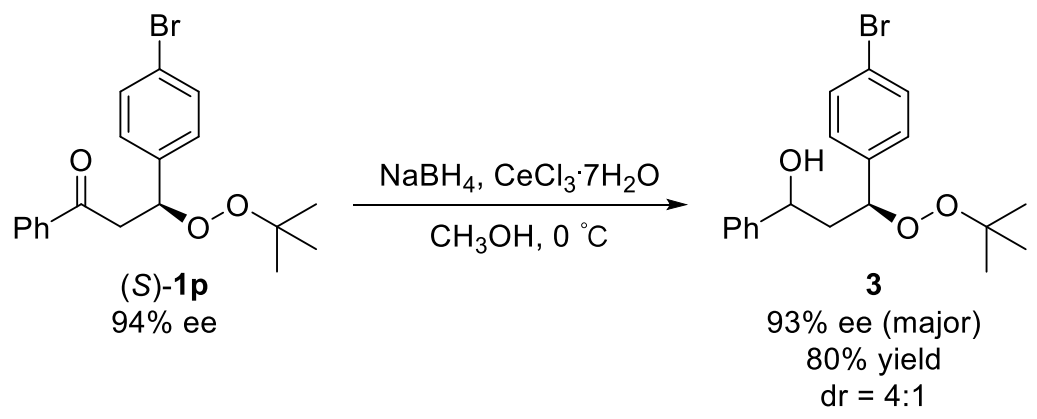

This reaction was carried out according to a literature method. ${ }^{2}(S)-\mathbf{1 p}\left(37.7 \mathrm{mg}, 0.1 \mathrm{mmol}, 1\right.$ equiv) and $\mathrm{CeCl}_{3} 7 \mathrm{H}_{2} \mathrm{O}$ (74.5 mg, $0.2 \mathrm{mmol}, 2$ equiv) were weighed and added into an oven dried flask, evacuated and backfilled with nitrogen (3 times). Freshly distilled $\mathrm{CH}_{3} \mathrm{OH}(1 \mathrm{~mL})$ was introduced, and the resulting mixture was cooled down to $0{ }^{\circ} \mathrm{C}$. $\mathrm{NaBH}_{4}\left(7.6 \mathrm{mg}, 0.2 \mathrm{mmol}, 2\right.$ equiv) was added slowly. The mixture was kept stirring for 5 minutes at $0{ }^{\circ} \mathrm{C}$, quenched with $\mathrm{H}_{2} \mathrm{O}$, extracted with EA, concentrated under reduced pressure and purified by flash column chromatography $\left(\mathrm{SiO}_{2}, \mathrm{PE} / \mathrm{EA}=6: 1\right)$ to afford the product 3 as a colorless oil $(30.3 \mathrm{mg}, 0.08 \mathrm{mmol}, 80 \%)(\mathrm{dr}=4: 1)$.<smiles>CC(C)(C)OOC(CC(=O)c1ccccc1)c1ccc(Br)cc1</smiles>

(S)-1p

$94 \%$ ee<smiles>NNc1ccc([N+](=O)[O-])cc1[N+](=O)[O-]</smiles>
$\underset{\mathrm{CH}_{3} \mathrm{OH}, 40{ }^{\circ} \mathrm{C}}{\stackrel{\mathrm{TsOH}}{\mathrm{MgSO}_{4}}}$

$\mathrm{Ph}$

$E / Z=5: 1$

4a-(E): $93 \%$ ee, $73 \%$ yield

4b-(Z): $88 \%$ ee, $14 \%$ yield

The reaction was carried out according to a literature method. ${ }^{3}$ To a mixture of $(S)-\mathbf{1 p}(37.7 \mathrm{mg}, 0.1 \mathrm{mmol}, 1 \mathrm{equiv})$, 2,4-dinitrophenyl hydrazine ( $19.8 \mathrm{mg}, 0.1 \mathrm{mmol}, 1$ equiv), TsOH ( $3.5 \mathrm{mg}, 0.02 \mathrm{mmol}, 0.2$ equiv), and $\mathrm{MgSO}_{4}(50$ $\mathrm{mg}), \mathrm{CH}_{3} \mathrm{OH}(0.5 \mathrm{~mL})$ was dropped into the mixture under nitrogen at room temperature. The resulting mixture was stirred at $40{ }^{\circ} \mathrm{C}$ for $6 \mathrm{~h}$. Solvent was evaporated in vacuum and the residue was purified by flash column chromatography $\left(\mathrm{SiO}_{2}, \mathrm{PE} / \mathrm{CH}_{2} \mathrm{Cl}_{2}=4: 1\right)$ to afford $48.4 \mathrm{mg}$ of $(E / Z)-4$ in a ratio of 5:1.

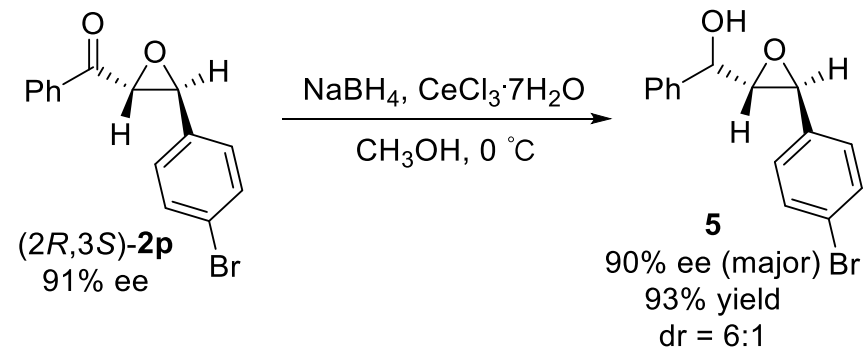

This reaction was carried out according to a literature method. ${ }^{2}(2 R, 3 S)-2 \mathbf{p}(30.3 \mathrm{mg}, 0.1 \mathrm{mmol}, 1$ equiv) and $\mathrm{CeCl}_{3} \cdot 7 \mathrm{H}_{2} \mathrm{O}$ (74.5 mg, $0.2 \mathrm{mmol}, 2$ equiv) were weighed and added into an oven dried flask, evacuated and backfilled with nitrogen (3 times). Freshly distilled $\mathrm{CH}_{3} \mathrm{OH}(1 \mathrm{~mL})$ was introduced, and the resulting mixture was cooled down to $0{ }^{\circ} \mathrm{C}$. $\mathrm{NaBH}_{4}\left(7.6 \mathrm{mg}, 0.2 \mathrm{mmol}, 2\right.$ equiv) was added slowly. The mixture was kept stirring for 5 minutes at $0{ }^{\circ} \mathrm{C}$, quenched with $\mathrm{H}_{2} \mathrm{O}$, extracted with EA, concentrated under reduced pressure and purified by flash column 
chromatography $\left(\mathrm{SiO}_{2}, \mathrm{PE} / \mathrm{EA}=6: 1\right)$ to afford the product 5 as a white solid $(27.5 \mathrm{mg}, 0.09 \mathrm{mmol}, 90 \%)(\mathrm{dr}=6: 1)$.

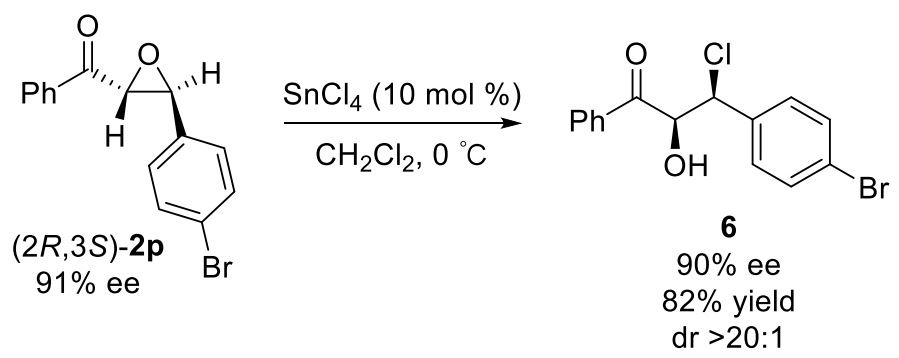

This reaction was carried out according to a literature method. ${ }^{4,5} \mathrm{SnCl}_{4}(10 \mathrm{~mol} \%)$ was added to a stirred solution of (2R,3S)-2p (30.3 mg, $0.1 \mathrm{mmol}, 1$ equiv) in $\mathrm{CH}_{2} \mathrm{Cl}_{2}(0.5 \mathrm{~mL})$ under nitrogen at $0{ }^{\circ} \mathrm{C}$. After TLC monitoring, the reaction mixture was poured in a $10 \%$ aqueous $\mathrm{Na}_{2} \mathrm{CO}_{3}$ solution and was extracted with $\mathrm{CH}_{2} \mathrm{Cl}_{2}$. The combined organic layer was washed with brine solution, dried over $\mathrm{Na}_{2} \mathrm{SO}_{4}$ and concentrated in vacuo to give the corresponding alcohol, which was purifed by silica gel column chromatography $\left(\mathrm{SiO}_{2}, \mathrm{PE} / \mathrm{EA}=6: 1\right)$ to obtain the product 6 as a white solid (27.8 mg, $0.08 \mathrm{mmol}, 82 \%)$.
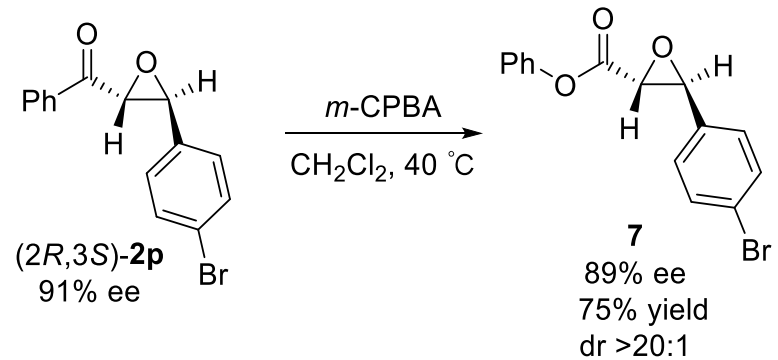

This reaction was carried out according to a literature method. ${ }^{6}$ A solution of $(2 R, 3 S)-2 \mathbf{p}(30.3 \mathrm{mg}, 0.1 \mathrm{mmol}, 1$ equiv) and $m$-CPBA (51.7 mg, $0.3 \mathrm{mmol}, 3$ equiv) in $\mathrm{CH}_{2} \mathrm{Cl}_{2}(0.5 \mathrm{~mL})$ under nitrogen was heated at reflux for $16 \mathrm{~h}$. The mixture was stirred with saturated aqueous $\mathrm{NaHSO}_{3}$ at $23{ }^{\circ} \mathrm{C}$ for $2 \mathrm{~h}$ and then washed with a saturated $\mathrm{NaHCO}_{3}$ solution and brine. After evaporating, the residue was purified by flash chromatography $\left(\mathrm{SiO}_{2}, \mathrm{PE} / \mathrm{EA}=10: 1\right)$ afford the product 7 as a white solid $(23.9 \mathrm{mg}, 75 \%)$. 


\section{VI. ${ }^{1} \mathrm{H},{ }^{13} \mathrm{C}$ NMR, HRMS data and HPLC traces of compounds (1a-s, 2a-s)}

(S)-3-(tert-butylperoxy)-1,3-diphenylropan-1-one (1a)<smiles>CC(C)(C)OOC(CC(=O)c1ccccc1)c1ccccc1</smiles>

(S)-1a

$82.1,80.5,43.8,26.2$.

${ }^{1} \mathbf{H}$ NMR $\left(400 \mathrm{MHz}, \mathrm{CDCl}_{3}\right): \delta 7.95(\mathrm{~d}, J=7.3 \mathrm{~Hz}, 2 \mathrm{H}), 7.55(\mathrm{t}, J=7.3 \mathrm{~Hz}, 1 \mathrm{H}), 7.49-7.38$ $(\mathrm{m}, 4 \mathrm{H}), 7.35$ (t, $J=7.3 \mathrm{~Hz}, 2 \mathrm{H}), 7.30(\mathrm{~d}, J=7.1 \mathrm{~Hz}, 1 \mathrm{H}), 5.60(\mathrm{t}, J=6.5 \mathrm{~Hz}, 1 \mathrm{H}), 3.78(\mathrm{dd}$, $J=16.3,7.1 \mathrm{~Hz}, 1 \mathrm{H}), 3.21(\mathrm{dd}, J=16.3,5.9 \mathrm{~Hz}, 1 \mathrm{H}), 1.16(\mathrm{~s}, 9 \mathrm{H})$.

${ }^{13} \mathrm{C}$ NMR $\left(100 \mathrm{MHz}, \mathrm{CDCl}_{3}\right): \delta 197.1,139.9,136.9,132.9,128.3,128.2,128.0,127.9,126.9$,

HRMS (ESI) m/z Calcd for $\left[\mathrm{C}_{19} \mathrm{H}_{22} \mathrm{NaO}_{3}, \mathrm{M}+\mathrm{Na}\right]^{+}: 321.1461$, Found: 321.1466

HPLC analysis: Chiralcel OJ-H $(\mathrm{Hexane} / i-\mathrm{PrOH}=99: 1$, flow rate $=1.0 \mathrm{~mL} / \mathrm{min}$, wave length $=254 \mathrm{~nm}), t_{\mathrm{R}}=12.360 \mathrm{~min}$ (minor), $t_{\mathrm{R}}=14.735 \mathrm{~min}$ (major).

Optical Rotation: $[\alpha]_{\mathrm{D}}^{20}=-46.7^{\circ}\left(c=1.0, \mathrm{CHCl}_{3}\right)$.

Physical properties: colorless oil; Yield: $46 \%, 13.7 \mathrm{mg}$; Reaction time: $47.0 \mathrm{~h}$.

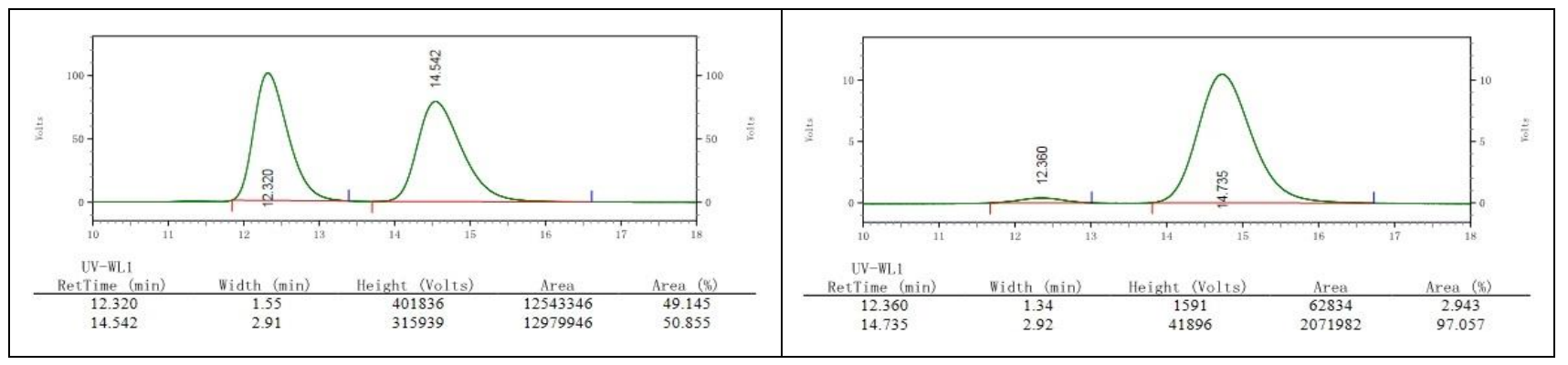

phenyl((2R,3S)-3-phenyloxiran-2-yl)methanone (2a)<smiles>O=C(c1ccccc1)[C@H]1O[C@H]1c1ccccc1</smiles>

$(2 R, 3 S)-2 a$ ${ }^{1} \mathbf{H}$ NMR (400 MHz, $\left.\mathrm{CDCl}_{3}\right): \delta 8.01(\mathrm{~d}, J=7.6 \mathrm{~Hz}, 2 \mathrm{H}), 7.62(\mathrm{t}, J=7.4 \mathrm{~Hz}, 1 \mathrm{H}), 7.48(\mathrm{t}, J=7.7$ $\mathrm{Hz}, 2 \mathrm{H}), 7.45-7.30(\mathrm{~m}, 5 \mathrm{H}), 4.30(\mathrm{~d}, J=1.9 \mathrm{~Hz}, 1 \mathrm{H}), 4.07(\mathrm{~d}, J=1.9 \mathrm{~Hz}, 1 \mathrm{H})$.

${ }^{13}$ C NMR (100 MHz, $\left.\mathrm{CDCl}_{3}\right): \delta 193.0,135.4,134.0,129.0,128.8,128.7,128.3,125.7,61.0,59.3$

HRMS (ESI) m/z Calcd for $\left[\mathrm{C}_{15} \mathrm{H}_{12} \mathrm{NaO}_{2}, \mathrm{M}+\mathrm{Na}\right]^{+}:$247.0730, Found: 247.0734.

HPLC analysis: Chiralcel AD-H $($ Hexane $/ i-\mathrm{PrOH}=85: 15$, flow rate $=1.0 \mathrm{~mL} / \mathrm{min}$, wave length $=254 \mathrm{~nm}), t_{\mathrm{R}}=8.994$ $\min$ (minor), $t_{\mathrm{R}}=9.486 \mathrm{~min}$ (major).

Optical Rotation: $[\alpha]_{\mathrm{D}}^{20}=-107.2^{\circ}\left(c=1.0, \mathrm{CHCl}_{3}\right)$.

Physical properties: white solid; Melting Point: $89.6-90.2{ }^{\circ} \mathrm{C}$; Yield: $49 \%, 11.0 \mathrm{mg}$; Reaction time: $47.0 \mathrm{~h}$.

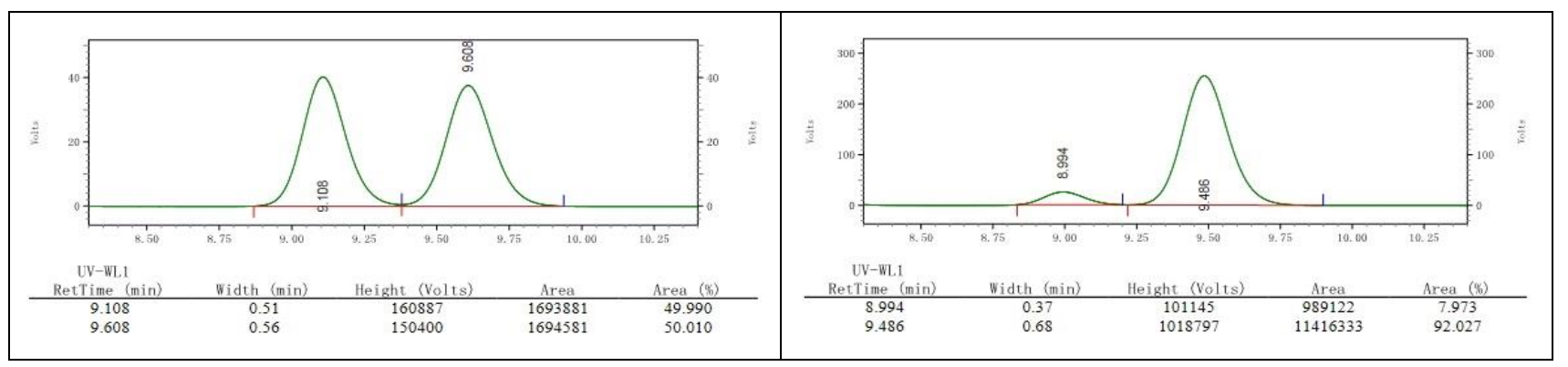


<smiles>Cc1ccc(C(=O)CC(OOC(C)(C)C)c2ccccc2)cc1</smiles>

(S)-1b

${ }^{1} \mathbf{H}$ NMR (400 MHz, $\left.\mathrm{CDCl}_{3}\right): \delta 7.85(\mathrm{~d}, J=8.1 \mathrm{~Hz}, 2 \mathrm{H}), 7.43(\mathrm{~d}, J=7.1 \mathrm{~Hz}, 2 \mathrm{H}), 7.33$ (t, $J=7.3 \mathrm{~Hz}, 2 \mathrm{H}), 7.30-7.25(\mathrm{~m}, 1 \mathrm{H}), 7.22(\mathrm{~d}, J=7.9 \mathrm{~Hz}, 2 \mathrm{H}), 5.60(\mathrm{t}, J=6.5 \mathrm{~Hz}, 1 \mathrm{H}), 3.73$ $(\mathrm{dd}, J=16.2,7.1 \mathrm{~Hz}, 1 \mathrm{H}), 3.17$ (dd, $J=16.2,5.9 \mathrm{~Hz}, 1 \mathrm{H}), 2.37$ (s, 3H), $1.16(\mathrm{~s}, 9 \mathrm{H})$.

${ }^{13}$ C NMR (100 MHz, $\left.\mathrm{CDCl}_{3}\right): \delta 197.0,143.9,140.1,134.6,129.2,128.3,128.0,127.1,82.3$, $80.7,43.8,26.3,21.6$.

HRMS (ESI) m/z Calcd for $\left[\mathrm{C}_{20} \mathrm{H}_{24} \mathrm{NaO}_{3}, \mathrm{M}+\mathrm{Na}\right]^{+}: 335.1618$, Found: 335.1617.

HPLC analysis: Chiralcel OJ-H $($ Hexane $/ i-\mathrm{PrOH}=99: 1$, flow rate $=1.0 \mathrm{~mL} / \mathrm{min}$, wave length $=254 \mathrm{~nm}), t_{\mathrm{R}}=11.521 \mathrm{~min}$ (minor), $t_{\mathrm{R}}=14.938 \mathrm{~min}$ (major).

Optical Rotation: $[\alpha]_{\mathrm{D}}^{20}=-37.9^{\circ}\left(c=1.0, \mathrm{CHCl}_{3}\right)$.

Physical properties: colorless oil; Yield: 45\%, $14.1 \mathrm{mg}$; Reaction time: $114.0 \mathrm{~h}$.

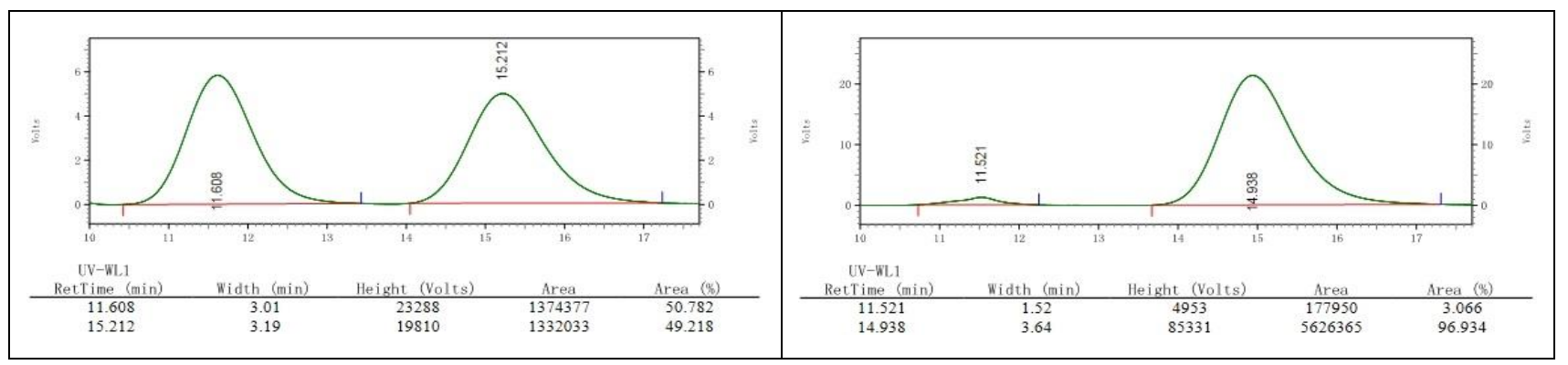

$((2 R, 3 S)-3-p h e n y l o x i r a n-2-y l)(p$-tolyl)methanone (2b)<smiles>Cc1ccc(C(=O)[C@@H]2O[C@H]2c2ccccc2)cc1</smiles>

$(2 R, 3 S)-\mathbf{2 b}$ ${ }^{1} \mathbf{H}$ NMR $\left(400 \mathrm{MHz}, \mathrm{CDCl}_{3}\right): \delta 7.89(\mathrm{~d}, J=8.1 \mathrm{~Hz}, 2 \mathrm{H}), 7.40-7.33(\mathrm{~m}, 5 \mathrm{H}), 7.25(\mathrm{~d}, J=8.0 \mathrm{~Hz}$, $2 \mathrm{H}), 4.27(\mathrm{~d}, J=1.5 \mathrm{~Hz}, 1 \mathrm{H}), 4.04(\mathrm{~d}, J=1.2 \mathrm{~Hz}, 1 \mathrm{H}), 2.39$ (s, 3H).

${ }^{13}$ C NMR $\left(100 \mathrm{MHz}, \mathrm{CDCl}_{3}\right): \delta 192.3,144.9,135.4,132.8,129.4,128.8,128.5,128.3,125.6$, 60.7, 59.1, 21.6.

HRMS (ESI) m/z Calcd for $\left[\mathrm{C}_{16} \mathrm{H}_{14} \mathrm{NaO}_{2}, \mathrm{M}+\mathrm{Na}\right]^{+}:$261.0886, Found: 261.0884.

HPLC analysis: Chiralcel AD-H $($ Hexane $/ i-\mathrm{PrOH}=95: 5$, flow rate $=1.0 \mathrm{~mL} / \mathrm{min}$, wave length $=254 \mathrm{~nm}), t_{\mathrm{R}}=18.771$ $\min \left(\right.$ minor), $t_{\mathrm{R}}=20.293 \min$ (major).

Optical Rotation: $[\alpha]_{\mathrm{D}}^{20}=-37.9^{\circ}\left(c=1.0, \mathrm{CHCl}_{3}\right)$.

Physical properties: white solid; Melting Point: $84.6-85.5^{\circ} \mathrm{C}$; Yield: 50\%, $11.9 \mathrm{mg}$; Reaction time: $114.0 \mathrm{~h}$.

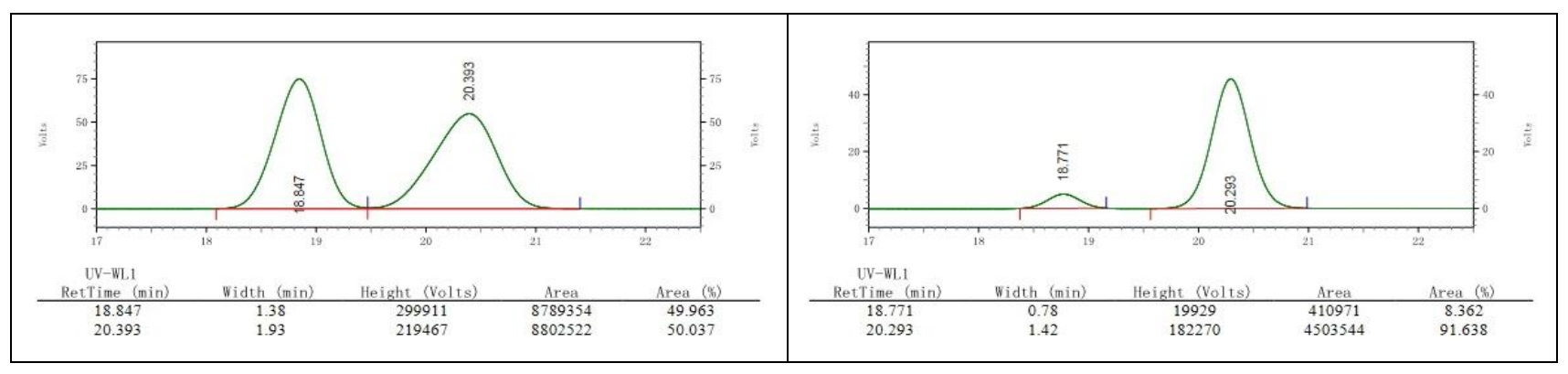


<smiles>CC(C)(C)OOC(CC(=O)c1ccc(C(C)(C)C)cc1)c1ccccc1</smiles>

${ }^{1}$ H NMR (400 MHz, $\left.\mathrm{CDCl}_{3}\right): \delta 7.89(\mathrm{~d}, J=8.4 \mathrm{~Hz}, 2 \mathrm{H}), 7.49-7.40(\mathrm{~m}, 4 \mathrm{H}), 7.33(\mathrm{t}, J=$ $7.4 \mathrm{~Hz}, 2 \mathrm{H}), 7.27(\mathrm{~d}, J=7.1 \mathrm{~Hz}, 1 \mathrm{H}), 5.61(\mathrm{t}, J=6.5 \mathrm{~Hz}, 1 \mathrm{H}), 3.74(\mathrm{dd}, J=16.3,7.0 \mathrm{~Hz}$, $1 \mathrm{H}), 3.21(\mathrm{dd}, J=16.3,6.1 \mathrm{~Hz}, 1 \mathrm{H}), 1.32(\mathrm{~s}, 9 \mathrm{H}), 1.17$ (s, 9H).

${ }^{13}$ C NMR (100 MHz, $\left.\mathrm{CDCl}_{3}\right): \delta 196.8,156.7,140.1,134.5,128.3,128.1,127.9,127.0$, $125.4,82.2,80.6,43.8,35.0,31.0,26.3$.

HRMS (ESI) m/z Calcd for $\left[\mathrm{C}_{23} \mathrm{H}_{30} \mathrm{NaO}_{3}, \mathrm{M}+\mathrm{Na}\right]^{+}:$377.2087, Found: 377.2086.

HPLC analysis: Chiralcel IA-H (Hexane $/ i-\mathrm{PrOH}=90: 10$, flow rate $=1.0 \mathrm{~mL} / \mathrm{min}$, wave length $=210 \mathrm{~nm}), t_{\mathrm{R}}=4.627 \mathrm{~min}$ (major), $t_{\mathrm{R}}=5.094 \mathrm{~min}$ (minor).

Optical Rotation: $[\alpha]_{\mathrm{D}}^{20}=-23.5^{\circ}\left(c=1.0, \mathrm{CHCl}_{3}\right)$.

Physical properties: pale yellow solid; Melting Point: $59.5-61.4^{\circ} \mathrm{C}$; Yield: $43 \%, 15.2 \mathrm{mg}$; Reaction time: $92.0 \mathrm{~h}$.

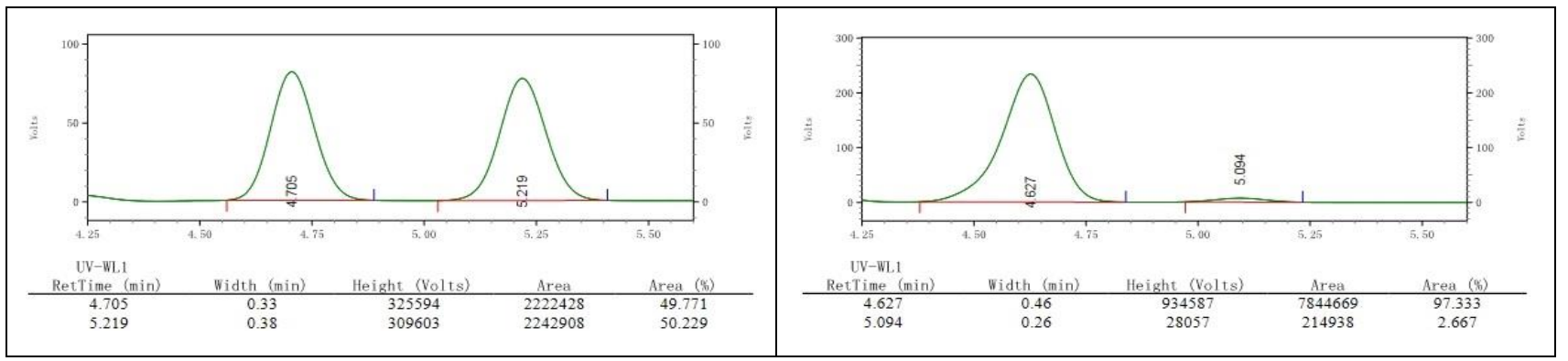

(4-(tert-butyl)phenyl)((2R,3S)-3-phenyloxiran-2-yl)methanone (2c)

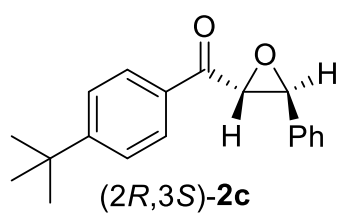
$(\mathrm{m}, 5 \mathrm{H}), 4.29(\mathrm{~d}, J=1.6 \mathrm{~Hz}, 1 \mathrm{H}), 4.06(\mathrm{~d}, J=1.9 \mathrm{~Hz}, 1 \mathrm{H}), 1.33(\mathrm{~s}, 9 \mathrm{H})$.

${ }^{13}$ C NMR (100 MHz, $\left.\mathrm{CDCl}_{3}\right): \delta 192.5,157.8,135.5,132.8,128.9,128.7,128.3,125.7,125.7$, 60.9, 59.2, 35.1, 30.9.

HRMS (ESI) m/z Calcd for $\left[\mathrm{C}_{19} \mathrm{H}_{20} \mathrm{NaO}_{2}, \mathrm{M}+\mathrm{Na}\right]^{+}: 303.1356$, Found: 303.1362.

HPLC analysis: Chiralcel IA-H (Hexane $/ i-\mathrm{PrOH}=90: 10$, flow rate $=1.0 \mathrm{~mL} / \mathrm{min}$, wave length $=210 \mathrm{~nm}), t_{\mathrm{R}}=7.700 \mathrm{~min}$ (minor), $t_{\mathrm{R}}=10.805 \mathrm{~min}$ (major).

Optical Rotation: $[\alpha]_{\mathrm{D}}^{20}=-82.7^{\circ}\left(c=1.0, \mathrm{CHCl}_{3}\right)$.

Physical properties: pale yellow oil; Yield: $52 \%, 14.6 \mathrm{mg}$; Reaction time: $92.0 \mathrm{~h}$.

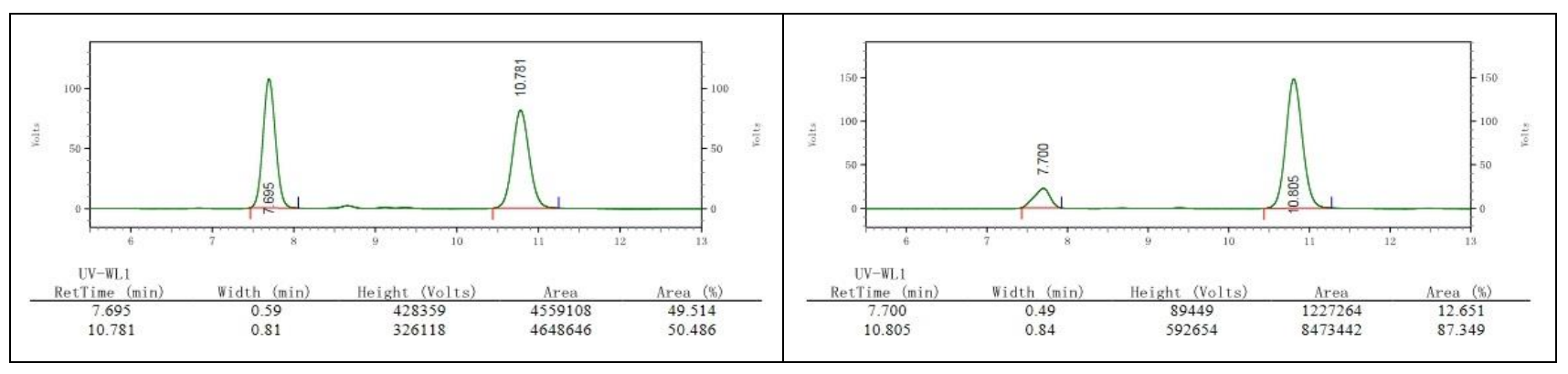


(S)-3-(tert-butylperoxy)-1-(3,4-dimethylphenyl)-3-phenylpropan-1-one (1d)<smiles>Cc1ccc(C(=O)CC(OOC(C)(C)C)c2ccccc2)cc1C</smiles>

${ }^{1} \mathbf{H}$ NMR $\left(400 \mathrm{MHz}, \mathrm{CDCl}_{3}\right): \delta 7.75-7.66(\mathrm{~m}, 2 \mathrm{H}), 7.43(\mathrm{~d}, J=7.2 \mathrm{~Hz}, 2 \mathrm{H}), 7.33(\mathrm{t}, J=7.3$ $\mathrm{Hz}, 2 \mathrm{H}), 7.30-7.25(\mathrm{~m}, 1 \mathrm{H}), 7.18(\mathrm{~d}, J=7.8 \mathrm{~Hz}, 1 \mathrm{H}), 5.60(\mathrm{t}, J=6.5 \mathrm{~Hz}, 1 \mathrm{H}), 3.73(\mathrm{dd}, J$ $=16.3,7.0 \mathrm{~Hz}, 1 \mathrm{H}), 3.18(\mathrm{dd}, J=16.3,6.0 \mathrm{~Hz}, 1 \mathrm{H}), 2.29(\mathrm{~s}, 6 \mathrm{H}), 1.18(\mathrm{~s}, 9 \mathrm{H})$.

${ }^{13}$ C NMR (100 MHz, $\left.\mathrm{CDCl}_{3}\right): \delta 197.2,142.6,140.2,136.8,135.1,129.7,129.4,128.3$, 128.0, 127.1, 126.0, 82.3, 80.7, 43.9, 26.4, 20.0, 19.7 .

HRMS (ESI) m/z Calcd for $\left[\mathrm{C}_{21} \mathrm{H}_{26} \mathrm{NaO}_{3}, \mathrm{M}+\mathrm{Na}\right]^{+}: 349.1774$, Found: 349.1780 .

HPLC analysis: Chiralcel IA-H $($ Hexane $/ i-\mathrm{PrOH}=95: 5$, flow rate $=1.0 \mathrm{~mL} / \mathrm{min}$, wave length $=254 \mathrm{~nm}), t_{\mathrm{R}}=4.942 \mathrm{~min}$ (major), $t_{\mathrm{R}}=5.278 \mathrm{~min}$ (minor).

Optical Rotation: $[\alpha]_{\mathrm{D}}^{20}=-32.7^{\circ}\left(c=1.0, \mathrm{CHCl}_{3}\right)$.

Physical properties: pale yellow solid; Melting Point: $61.5-62.8^{\circ} \mathrm{C}$; Yield: $48 \%, 15.7 \mathrm{mg}$; Reaction time: $88.0 \mathrm{~h}$.

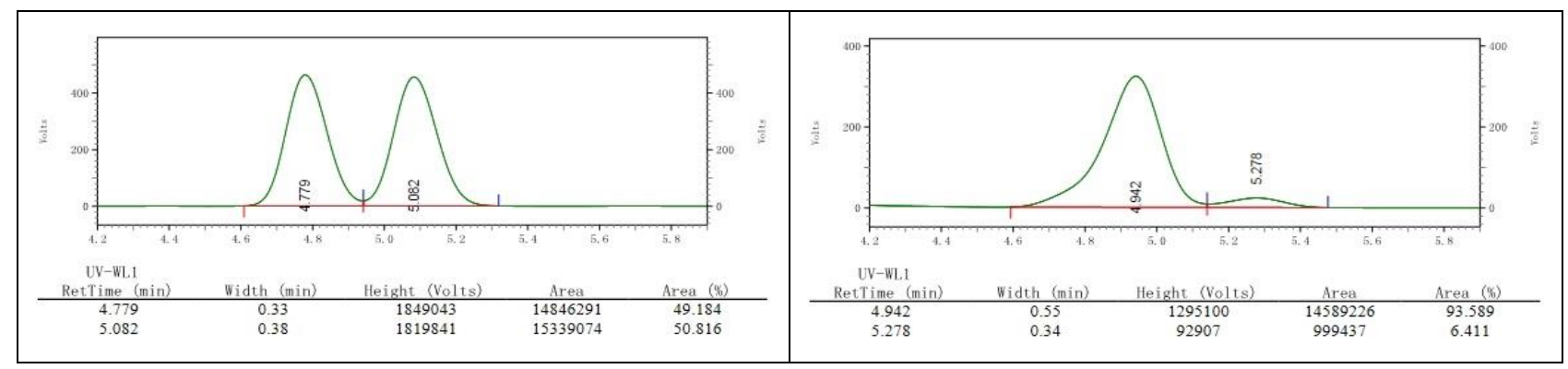

(3,4-dimethylphenyl)((2R,3S)-3-phenyloxiran-2-yl)methanone (2d)

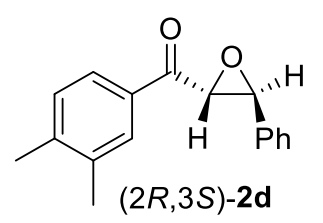

${ }^{1} \mathbf{H}$ NMR $\left(400 \mathrm{MHz}, \mathrm{CDCl}_{3}\right): \delta 7.78(\mathrm{~s}, 1 \mathrm{H}), 7.74(\mathrm{~d}, J=7.8 \mathrm{~Hz}, 1 \mathrm{H}), 7.42-7.34(\mathrm{~m}, 5 \mathrm{H}), 7.22$ $(\mathrm{d}, J=7.8 \mathrm{~Hz}, 1 \mathrm{H}), 4.28(\mathrm{~d}, J=1.8 \mathrm{~Hz}, 1 \mathrm{H}), 4.06(\mathrm{~d}, J=1.6 \mathrm{~Hz}, 1 \mathrm{H}), 2.31(\mathrm{~d}, J=6.4 \mathrm{~Hz}, 6 \mathrm{H})$. ${ }^{13}$ C NMR (100 MHz, $\left.\mathrm{CDCl}_{3}\right): \delta 192.7,143.8,137.3,135.6,133.4,130.0,129.3,128.9,128.7$, 126.0, 125.7, 60.7, 59.3, 20.1, 19.7 .

HRMS (ESI) m/z Calcd for $\left[\mathrm{C}_{17} \mathrm{H}_{16} \mathrm{NaO}_{2}, \mathrm{M}+\mathrm{Na}\right]^{+}: 275.1043$, Found: 275.1047.

HPLC analysis: Chiralcel IA-H $($ Hexane $/ i-\mathrm{PrOH}=95: 5$, flow rate $=1.0 \mathrm{~mL} / \mathrm{min}$, wave length $=254 \mathrm{~nm}), t_{\mathrm{R}}=12.329 \mathrm{~min}$ (minor), $t_{\mathrm{R}}=14.165 \mathrm{~min}$ (major).

Optical Rotation: $[\alpha]_{\mathrm{D}}^{20}=-109.8^{\circ}\left(c=1.0, \mathrm{CHCl}_{3}\right)$.

Physical properties: white solid; Melting Point: $88.9-90.1^{\circ} \mathrm{C}$; Yield: $48 \%, 12.1 \mathrm{mg}$; Reaction time: $88.0 \mathrm{~h}$.

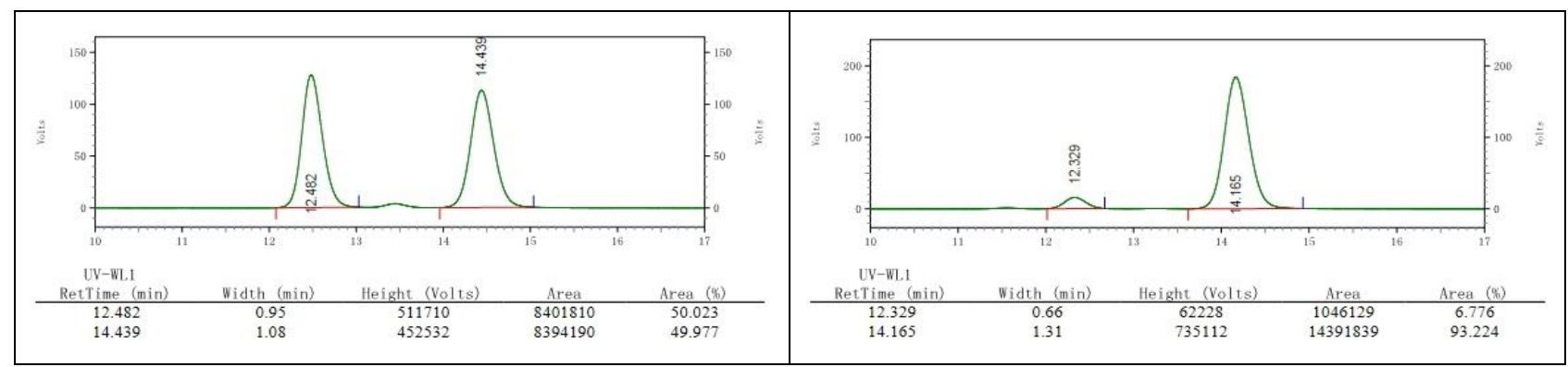


<smiles>COc1cccc(C(=O)CC(OOC(C)(C)C)c2ccccc2)c1</smiles>

${ }^{1} \mathbf{H}$ NMR $\left(400 \mathrm{MHz}, \mathrm{CDCl}_{3}\right): \delta 7.54(\mathrm{~d}, J=7.6 \mathrm{~Hz}, 1 \mathrm{H}), 7.48-7.40(\mathrm{~m}, 3 \mathrm{H}), 7.39-7.32(\mathrm{~m}$, $3 \mathrm{H}), 7.30(\mathrm{~d}, J=7.2 \mathrm{~Hz}, 1 \mathrm{H}), 7.10(\mathrm{dd}, J=8.2,2.5 \mathrm{~Hz}, 1 \mathrm{H}), 5.59(\mathrm{t}, J=6.5 \mathrm{~Hz}, 1 \mathrm{H}), 3.83(\mathrm{~s}$, $3 \mathrm{H}), 3.76(\mathrm{dd}, J=16.3,7.0 \mathrm{~Hz}, 1 \mathrm{H}), 3.22(\mathrm{dd}, J=16.3,6.0 \mathrm{~Hz}, 1 \mathrm{H}), 1.17$ (s, 9H).

${ }^{13} \mathrm{C}$ NMR $\left(100 \mathrm{MHz}, \mathrm{CDCl}_{3}\right): \delta 196.7,159.5,139.8,138.2,129.2,128.1,127.8,126.8,120.6$, $119.3,112.1,82.0,80.3,54.9,43.8,26.1$.

HRMS (ESI) m/z Calcd for $\left[\mathrm{C}_{20} \mathrm{H}_{24} \mathrm{NaO}_{4}, \mathrm{M}+\mathrm{Na}\right]^{+}: 351.1567$, Found: 351.1575.

HPLC analysis: Chiralcel AD-H $($ Hexane $/ i-\mathrm{PrOH}=97: 3$, flow rate $=1.0 \mathrm{~mL} / \mathrm{min}$, wave length $=254 \mathrm{~nm}), t_{\mathrm{R}}=7.280 \mathrm{~min}$ (major), $t_{\mathrm{R}}=7.996 \mathrm{~min}$ (minor).

Optical Rotation: $[\alpha]_{\mathrm{D}}^{20}=-29.6^{\circ}\left(c=1.0, \mathrm{CHCl}_{3}\right)$.

Physical properties: colorless oil; Yield: 46\%, $15.1 \mathrm{mg}$; Reaction time: $48.0 \mathrm{~h}$.

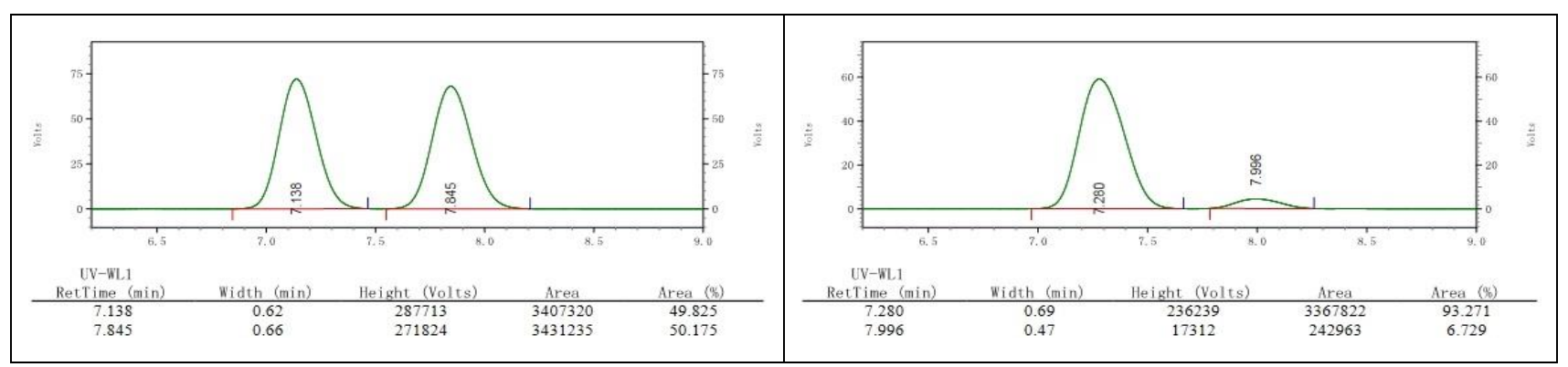

(3-methoxyphenyl)((2R,3S)-3-phenyloxiran-2-yl)methanone (2e)<smiles>COc1cccc(C(=O)[C@@H]2O[C@H]2c2ccccc2)c1</smiles>

$(2 R, 3 S)-2 \mathbf{e}$
${ }^{1} \mathbf{H}$ NMR (400 MHz, $\left.\mathrm{CDCl}_{3}\right): \delta 7.57(\mathrm{~d}, J=7.6 \mathrm{~Hz}, 1 \mathrm{H}), 7.53(\mathrm{t}, J=2.0 \mathrm{~Hz}, 1 \mathrm{H}), 7.43-7.34(\mathrm{~m}$, $6 \mathrm{H}), 7.16(\mathrm{dd}, J=8.2,2.3 \mathrm{~Hz}, 1 \mathrm{H}), 4.28(\mathrm{~d}, J=1.6 \mathrm{~Hz}, 1 \mathrm{H}), 4.07(\mathrm{~d}, J=1.9 \mathrm{~Hz}, 1 \mathrm{H}), 3.84(\mathrm{~s}, 3 \mathrm{H})$.

${ }^{13}$ C NMR (100 MHz, $\left.\mathrm{CDCl}_{3}\right): \delta 192.7,159.8,136.5,135.3,129.7,128.9,128.6,125.6,120.8$, $120.4,112.2,60.8,59.3,55.3$.

HRMS (ESI) m/z Calcd for $\left[\mathrm{C}_{16} \mathrm{H}_{14} \mathrm{NaO}_{3}, \mathrm{M}+\mathrm{Na}\right]^{+}:$277.0835, Found: 277.0840 .

HPLC analysis: Chiralcel IA-H (Hexane $/ i-\mathrm{PrOH}=95: 5$, flow rate $=1.0 \mathrm{~mL} / \mathrm{min}$, wave length $=254 \mathrm{~nm}), t_{\mathrm{R}}=14.592 \mathrm{~min}$ (minor), $t_{\mathrm{R}}=15.744$ min (major).

Optical Rotation: $[\alpha]_{\mathrm{D}}^{20}=-83.1^{\circ}\left(c=1.0, \mathrm{CHCl}_{3}\right)$.

Physical properties: colorless oil; Yield: $44 \%, 11.2 \mathrm{mg}$; Reaction time: $48.0 \mathrm{~h}$.

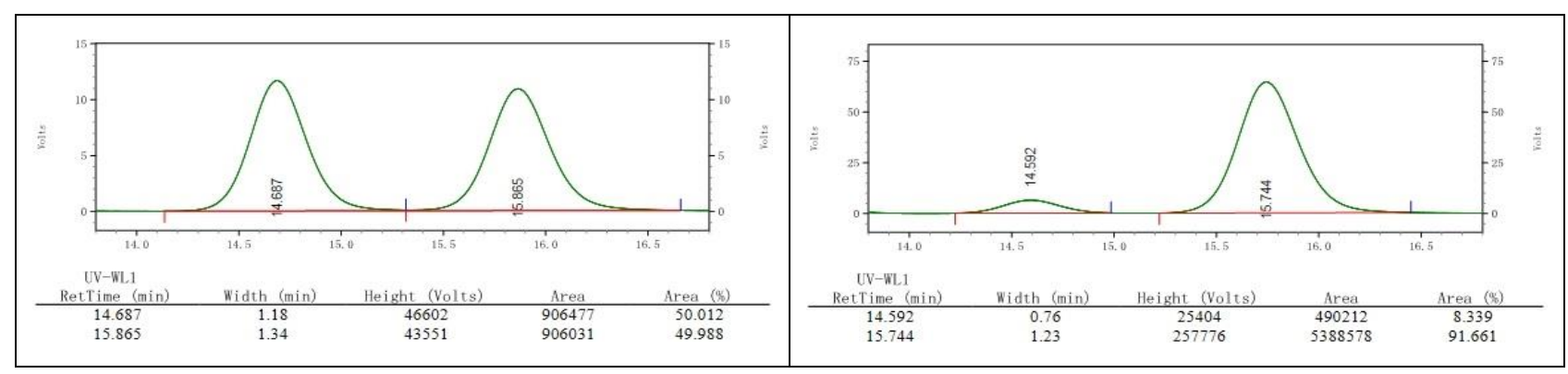


<smiles>COc1cc(C(=O)CC(OOC(C)(C)C)c2ccccc2)cc(OC)c1OC</smiles>

${ }^{1} \mathbf{H}$ NMR $\left(400 \mathrm{MHz}, \mathrm{CDCl}_{3}\right): \delta 7.43(\mathrm{~d}, J=7.2 \mathrm{~Hz}, 2 \mathrm{H}), 7.35(\mathrm{t}, J=7.3 \mathrm{~Hz}, 2 \mathrm{H}), 7.32-$ $7.28(\mathrm{~m}, 1 \mathrm{H}), 7.20(\mathrm{~s}, 2 \mathrm{H}), 5.59(\mathrm{t}, J=6.5 \mathrm{~Hz}, 1 \mathrm{H}), 3.91(\mathrm{~s}, 3 \mathrm{H}), 3.89(\mathrm{~s}, 6 \mathrm{H}), 3.74(\mathrm{dd}$, $J=16.2,6.6 \mathrm{~Hz}, 1 \mathrm{H}), 3.24(\mathrm{dd}, J=16.2,6.4 \mathrm{~Hz}, 1 \mathrm{H}), 1.20(\mathrm{~s}, 9 \mathrm{H})$.

${ }^{13}$ C NMR (100 MHz, $\left.\mathrm{CDCl}_{3}\right): \delta 196.1,152.9,142.5,139.8,132.3,128.4,128.1,127.0$, 105.6, 82.4, 80.8, 60.9, 56.2, 43.7, 26.3.

HRMS (ESI) m/z Calcd for $\left[\mathrm{C}_{22} \mathrm{H}_{28} \mathrm{NaO}_{6}, \mathrm{M}+\mathrm{Na}\right]^{+}: 411.1778$, Found: 411.1786.

HPLC analysis: Chiralcel AD-H $($ Hexane $/ i$-PrOH $=97: 3$, flow rate $=1.0 \mathrm{~mL} / \mathrm{min}$, wave length $=254 \mathrm{~nm}), t_{\mathrm{R}}=14.713$ $\min$ (major), $t_{\mathrm{R}}=15.721 \mathrm{~min}$ (minor).

Optical Rotation: $[\alpha]_{\mathrm{D}}^{20}=-22.5^{\circ}\left(c=1.0, \mathrm{CHCl}_{3}\right)$.

Physical properties: white solid; Melting Point: $93.1-94.8^{\circ} \mathrm{C}$; Yield: $47 \%, 18.3 \mathrm{mg}$; Reaction time: $65.0 \mathrm{~h}$.

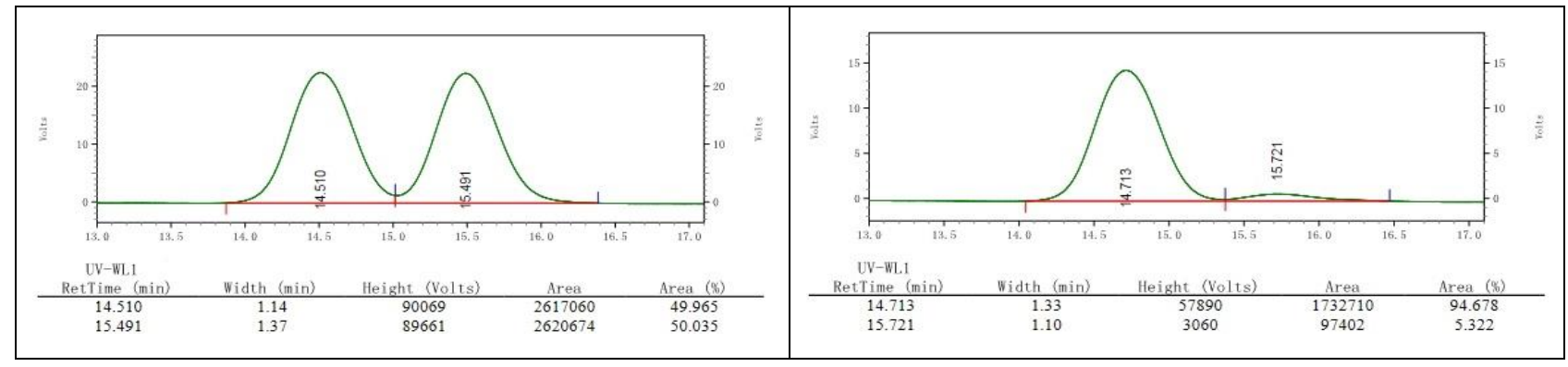

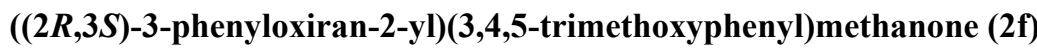<smiles>CC#[R6]OCC</smiles>

${ }^{1} \mathbf{H}$ NMR (400 MHz, $\left.\mathrm{CDCl}_{3}\right): \delta 7.43-7.36(\mathrm{~m}, 5 \mathrm{H}), 7.30(\mathrm{~s}, 2 \mathrm{H}), 4.22(\mathrm{~d}, J=1.8 \mathrm{~Hz}, 1 \mathrm{H})$, $4.10(\mathrm{~d}, J=1.7 \mathrm{~Hz}, 1 \mathrm{H}), 3.93(\mathrm{~s}, 3 \mathrm{H}), 3.89(\mathrm{~s}, 6 \mathrm{H})$.

${ }^{13}$ C NMR (100 MHz, $\left.\mathrm{CDCl}_{3}\right): \delta 191.6,153.0,143.3,135.3,130.3,128.8,128.6,125.5,105.8$, $60.9,60.8,59.0,56.1$.

HRMS (ESI) m/z Calcd for $\left[\mathrm{C}_{18} \mathrm{H}_{18} \mathrm{NaO}_{5}, \mathrm{M}+\mathrm{Na}\right]^{+}:$377.1046, Found: 377.1051.

HPLC analysis: Chiralcel AD-H $($ Hexane $/ i-\mathrm{PrOH}=80: 20$, flow rate $=1.0 \mathrm{~mL} / \mathrm{min}$, wave length $=254 \mathrm{~nm}), t_{\mathrm{R}}=11.473$ $\min \left(\right.$ minor), $t_{\mathrm{R}}=12.511 \min$ (major).

Optical Rotation: $[\alpha]_{\mathrm{D}}^{20}=-94.5^{\circ}\left(c=1.0, \mathrm{CHCl}_{3}\right)$.

Physical properties: white solid; Melting Point: $122.1-122.8^{\circ} \mathrm{C}$; Yield: $45 \%, 14.1 \mathrm{mg}$; Reaction time: $65.0 \mathrm{~h}$.

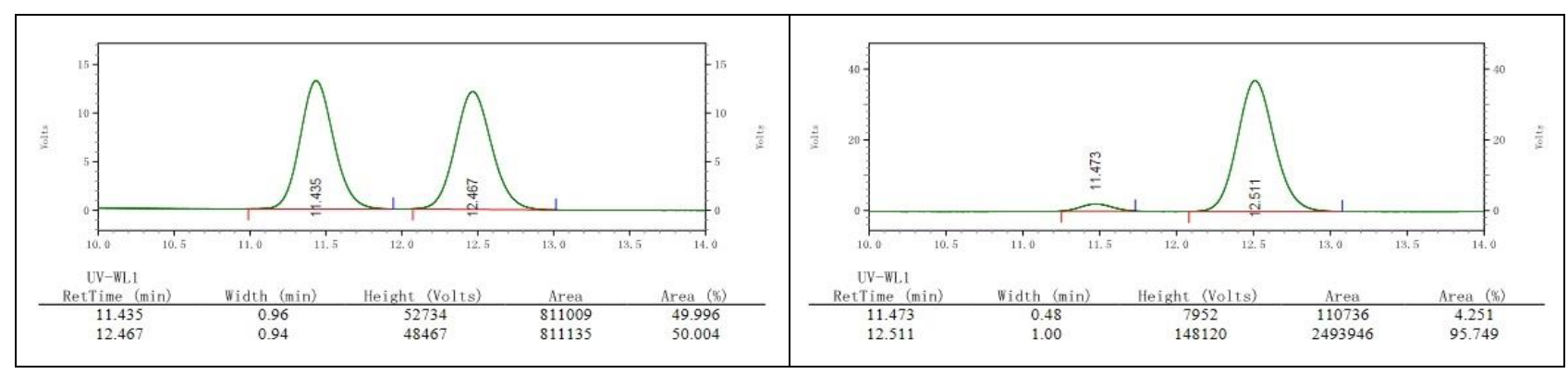


<smiles>CC(C)(C)OOC(CC(=O)c1ccc(Cl)cc1)c1ccccc1</smiles>

${ }^{1} \mathbf{H}$ NMR $\left(400 \mathrm{MHz}, \mathrm{CDCl}_{3}\right): \delta 7.86(\mathrm{~d}, J=8.5 \mathrm{~Hz}, 2 \mathrm{H}), 7.42(\mathrm{~d}, J=7.3 \mathrm{~Hz}, 2 \mathrm{H}), 7.37$ $(\mathrm{d}, J=8.5 \mathrm{~Hz}, 2 \mathrm{H}), 7.32(\mathrm{t}, J=7.3 \mathrm{~Hz}, 2 \mathrm{H}), 7.28-7.22(\mathrm{~m}, 1 \mathrm{H}), 5.59(\mathrm{t}, J=6.4 \mathrm{~Hz}, 1 \mathrm{H})$, $3.72(\mathrm{dd}, J=16.1,7.3 \mathrm{~Hz}, 1 \mathrm{H}), 3.13(\mathrm{dd}, J=16.1,5.6 \mathrm{~Hz}, 1 \mathrm{H}), 1.15(\mathrm{~s}, 9 \mathrm{H})$.

${ }^{13}$ C NMR (100 MHz, $\left.\mathrm{CDCl}_{3}\right): \delta 196.0,139.6,139.3,135.3,129.5,128.6,128.3,128.0$,

$126.9,82.1,80.6,43.8,26.2$.

HRMS (ESI) $\mathrm{m} / \mathrm{z}$ Calcd for $\left[\mathrm{C}_{19} \mathrm{H}_{21} \mathrm{ClNaO}_{3}, \mathrm{M}+\mathrm{Na}^{+}\right.$: 355.1071, Found: 355.1070.

HPLC analysis: Chiralcel OJ-H (Hexane $/ i-\mathrm{PrOH}=99: 1$, flow rate $=1.0 \mathrm{~mL} / \mathrm{min}$, wave length $=254 \mathrm{~nm}), t_{\mathrm{R}}=14.207 \mathrm{~min}$ (minor), $t_{\mathrm{R}}=19.057 \mathrm{~min}$ (major).

Optical Rotation: $[\alpha]_{\mathrm{D}}^{20}=-19.8^{\circ}\left(c=1.0, \mathrm{CHCl}_{3}\right)$.

Physical properties: white solid; Melting Point: $57.3-58.9^{\circ} \mathrm{C}$; Yield: $44 \%, 14.6 \mathrm{mg}$; Reaction time: $64.0 \mathrm{~h}$.

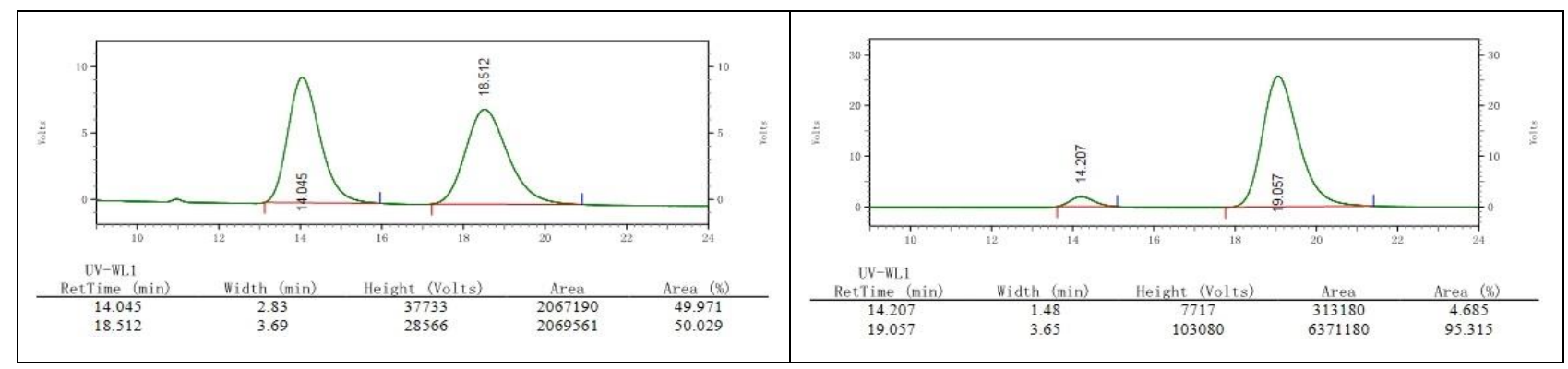

(4-chlorophenyl)((2R,3S)-3-phenyloxiran-2-yl)methanone (2g)<smiles>O=C(c1ccc(Cl)cc1)C1O[C@H]1c1ccccc1</smiles>

$(2 R, 3 S)-2 \mathrm{~g}$

${ }^{1} \mathbf{H}$ NMR $\left(400 \mathrm{MHz}, \mathrm{CDCl}_{3}\right): \delta 7.95(\mathrm{~d}, J=8.4 \mathrm{~Hz}, 2 \mathrm{H}), 7.44(\mathrm{~d}, J=8.3 \mathrm{~Hz}, 2 \mathrm{H}), 7.40-7.33$ $(\mathrm{m}, 5 \mathrm{H}), 4.24(\mathrm{~d}, J=1.7 \mathrm{~Hz}, 1 \mathrm{H}), 4.06(\mathrm{~d}, J=1.8 \mathrm{~Hz}, 1 \mathrm{H})$.

${ }^{13}$ C NMR (100 MHz, $\left.\mathrm{CDCl}_{3}\right): \delta 191.9,140.4,135.1,133.5,129.7,129.1,129.0,128.7,125.7$, 60.9, 59.3.

HRMS (ESI) $\mathrm{m} / \mathrm{z}$ Calcd for $\left[\mathrm{C}_{15} \mathrm{H}_{11} \mathrm{ClNaO}_{2}, \mathrm{M}+\mathrm{Na}^{+}:\right.$281.0340, Found: 281.0347.

HPLC analysis: Chiralcel AD-H (Hexane $/ i-\mathrm{PrOH}=80: 20$, flow rate $=1.0 \mathrm{~mL} / \mathrm{min}$, wave length $=254 \mathrm{~nm}), t_{\mathrm{R}}=9.652$ $\min$ (major), $t_{\mathrm{R}}=11.183 \min$ (minor).

Optical Rotation: $[\alpha]_{\mathrm{D}}^{20}=-61.7^{\circ}\left(c=1.0, \mathrm{CHCl}_{3}\right)$.

Physical properties: white solid; Melting Point: $125.5-126.1^{\circ} \mathrm{C}$; Yield: $50 \%, 12.9 \mathrm{mg}$; Reaction time: $64.0 \mathrm{~h}$.

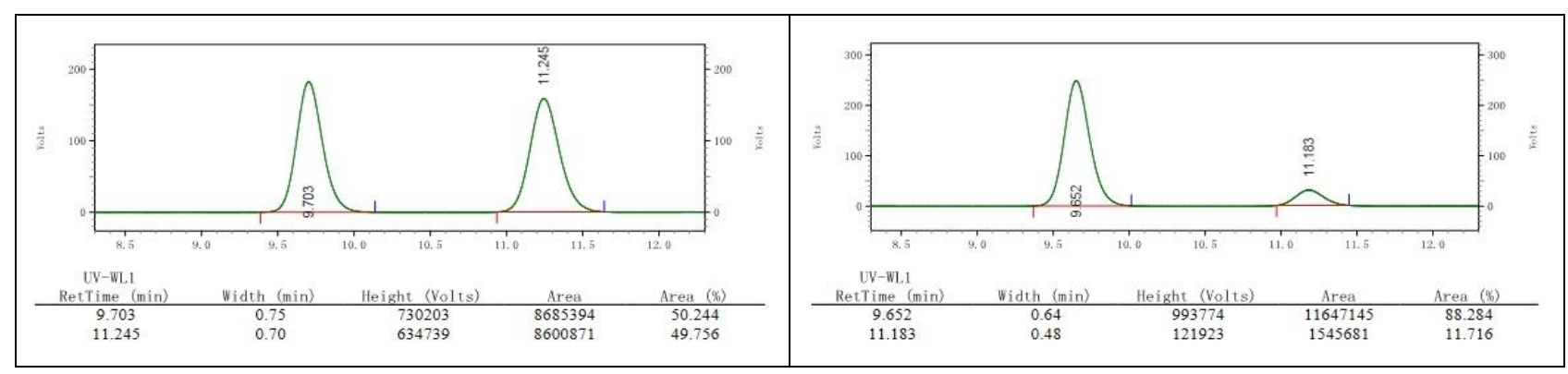


<smiles>CC(C)(C)OOC(CC(=O)c1ccc(Br)cc1)c1ccccc1</smiles>

${ }^{1} \mathbf{H}$ NMR $\left(400 \mathrm{MHz}, \mathrm{CDCl}_{3}\right): \delta 7.81(\mathrm{~d}, J=8.5 \mathrm{~Hz}, 2 \mathrm{H}), 7.58(\mathrm{~d}, J=8.5 \mathrm{~Hz}, 2 \mathrm{H}), 7.42$ $(\mathrm{d}, J=6.5 \mathrm{~Hz}, 2 \mathrm{H}), 7.37-7.32(\mathrm{~m}, 2 \mathrm{H}), 7.31-7.25(\mathrm{~m}, 1 \mathrm{H}), 5.57(\mathrm{t}, J=6.4 \mathrm{~Hz}, 1 \mathrm{H}), 3.73$ (dd, $J=16.1,7.2 \mathrm{~Hz}, 1 \mathrm{H}), 3.15(\mathrm{dd}, J=16.1,5.7 \mathrm{~Hz}, 1 \mathrm{H}), 1.15(\mathrm{~s}, 9 \mathrm{H})$.

${ }^{13} \mathbf{C}$ NMR (100 MHz, $\left.\mathrm{CDCl}_{3}\right): \delta 196.5,139.6,135.8,131.8,129.7,128.4,128.2,128.2$,

$127.0,82.3,80.8,43.9,26.3$.

HRMS (ESI) $\mathrm{m} / \mathrm{z}$ Calcd for $\left[\mathrm{C}_{19} \mathrm{H}_{21} \mathrm{BrNaO}_{3}, \mathrm{M}+\mathrm{Na}\right]^{+}:$399.0566, Found: 399.0567.

HPLC analysis: Chiralcel OJ-H (Hexane $/ i-\mathrm{PrOH}=99: 1$, flow rate $=1.0 \mathrm{~mL} / \mathrm{min}$, wave length $=254 \mathrm{~nm}), t_{\mathrm{R}}=18.524 \mathrm{~min}$ (minor), $t_{\mathrm{R}}=22.997 \min$ (major).

Optical Rotation: $[\alpha]_{\mathrm{D}}^{20}=-34.2^{\circ}\left(c=1.0, \mathrm{CHCl}_{3}\right)$.

Physical properties: white solid; Melting Point: $71.2-72.1^{\circ} \mathrm{C}$; Yield: $43 \%, 16.2 \mathrm{mg}$; Reaction time: $65.5 \mathrm{~h}$.

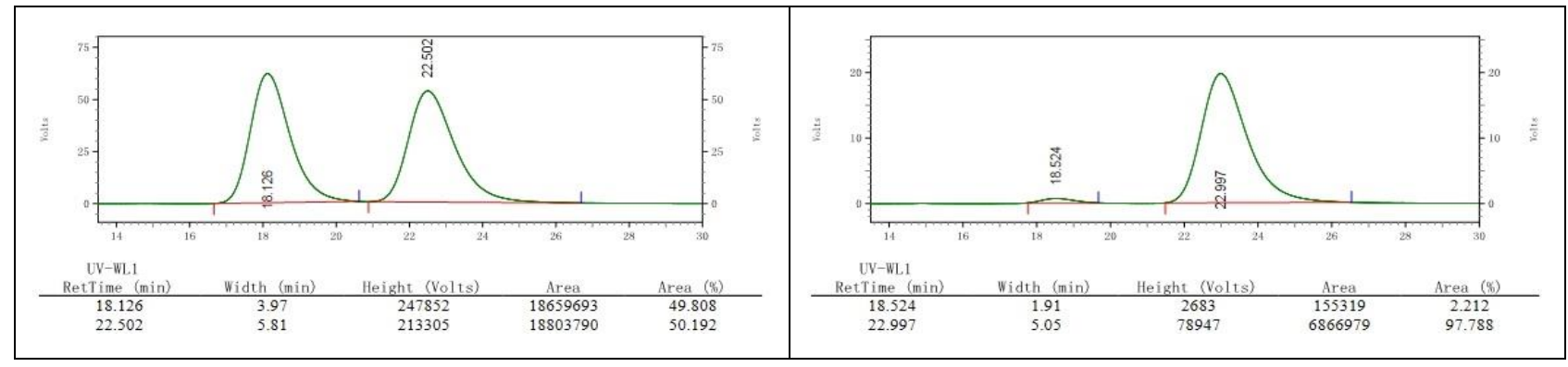

(4-bromophenyl)((2R,3S)-3-phenyloxiran-2-yl)methanone (2h)<smiles>O=C(c1ccc(Br)cc1)[C@H]1O[C@H]1c1ccccc1</smiles>

$(2 R, 3 S)-2 \mathbf{h}$

${ }^{1} \mathbf{H}$ NMR $\left(400 \mathrm{MHz}, \mathrm{CDCl}_{3}\right): \delta 7.87(\mathrm{~d}, J=8.0 \mathrm{~Hz}, 2 \mathrm{H}), 7.62(\mathrm{~d}, J=8.0 \mathrm{~Hz}, 2 \mathrm{H}), 7.42-7.34$ (m, 5H), $4.23(\mathrm{~s}, 1 \mathrm{H}), 4.06(\mathrm{~s}, 1 \mathrm{H})$.

${ }^{13} \mathbf{C}$ NMR $\left(100 \mathrm{MHz}, \mathrm{CDCl}_{3}\right): \delta 192.2,135.1,134.0,132.1,129.8,129.3,129.1,128.7,125.7$, 60.9, 59.3 .

HRMS (ESI) $\mathrm{m} / \mathrm{z}$ Calcd for $\left[\mathrm{C}_{15} \mathrm{H}_{11} \mathrm{BrNaO}_{2}, \mathrm{M}+\mathrm{Na}\right]^{+}: 324.9835$, Found: 324.9841.

HPLC analysis: Chiralcel AD-H (Hexane $/ i-\mathrm{PrOH}=85: 15$, flow rate $=1.0 \mathrm{~mL} / \mathrm{min}$, wave length $=254 \mathrm{~nm}), t_{\mathrm{R}}=10.795$ $\min$ (major), $t_{\mathrm{R}}=12.388 \min$ (minor).

Optical Rotation: $[\alpha]_{\mathrm{D}}^{20}=-96.1^{\circ}\left(c=1.0, \mathrm{CHCl}_{3}\right)$.

Physical properties: white solid; Melting Point: $124.5-125.1^{\circ} \mathrm{C}$; Yield: $52 \%, 15.8 \mathrm{mg}$; Reaction time: $65.5 \mathrm{~h}$.

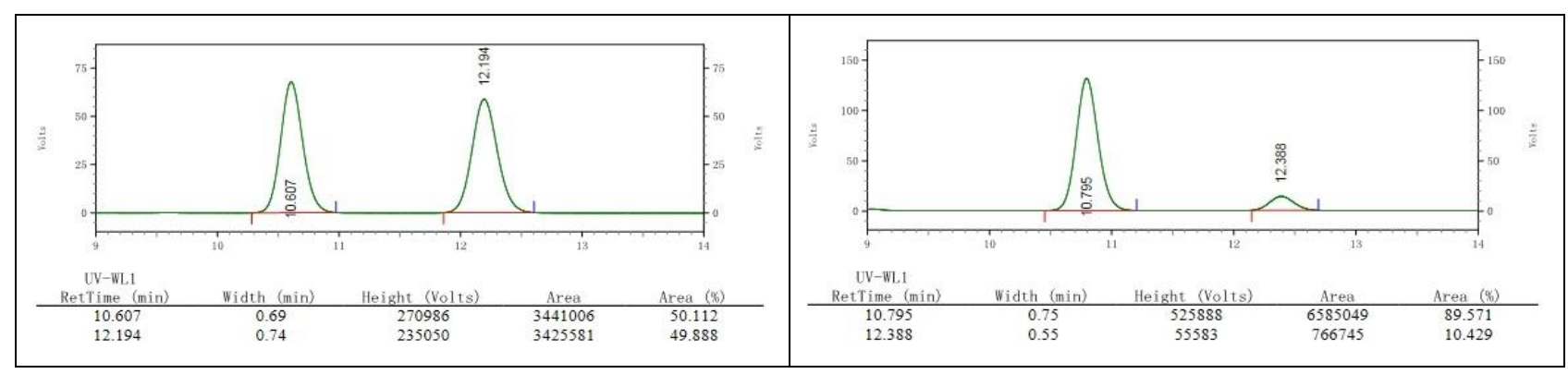


<smiles>CC(C)(C)OOC(CC(=O)c1ccc2ccccc2c1)c1ccccc1</smiles>
(S)-1i

${ }^{1} \mathbf{H}$ NMR $\left(400 \mathrm{MHz}, \mathrm{CDCl}_{3}\right): \delta 8.46(\mathrm{~s}, 1 \mathrm{H}), 8.01(\mathrm{~d}, J=8.6 \mathrm{~Hz}, 1 \mathrm{H}), 7.92(\mathrm{~d}, J=7.9$ $\mathrm{Hz}, 1 \mathrm{H}), 7.84(\mathrm{t}, J=8.0 \mathrm{~Hz}, 2 \mathrm{H}), 7.58-7.50(\mathrm{~m}, 2 \mathrm{H}), 7.47$ (d, $J=7.4 \mathrm{~Hz}, 2 \mathrm{H}), 7.35$ (t, $J=7.3 \mathrm{~Hz}, 2 \mathrm{H}), 7.31-7.26(\mathrm{~m}, 1 \mathrm{H}), 5.67(\mathrm{t}, J=6.4 \mathrm{~Hz}, 1 \mathrm{H}), 3.91(\mathrm{dd}, J=16.1,7.1 \mathrm{~Hz}$, $1 \mathrm{H}), 3.32(\mathrm{dd}, J=16.1,5.8 \mathrm{~Hz}, 1 \mathrm{H}), 1.17$ (s, 9H).

${ }^{13} \mathrm{C} \mathrm{NMR}\left(100 \mathrm{MHz}, \mathrm{CDCl}_{3}\right): \delta 197.0,139.9,135.3,134.2,132.2,129.8,129.4,128.3,128.2,128.2,127.9,127.5,127.0$, $126.5,123.7,82.2,80.6,43.9,26.2$.

HRMS (ESI) m/z Calcd for $\left[\mathrm{C}_{23} \mathrm{H}_{24} \mathrm{NaO}_{3}, \mathrm{M}+\mathrm{Na}\right]^{+}: 371.1618$, Found: 371.1623.

HPLC analysis: Chiralcel IA-H $($ Hexane $/ i-\mathrm{PrOH}=90: 10$, flow rate $=1.0 \mathrm{~mL} / \mathrm{min}$, wave length $=210 \mathrm{~nm}), t_{\mathrm{R}}=5.084 \mathrm{~min}$ (major), $t_{\mathrm{R}}=5.646 \mathrm{~min}$ (minor).

Optical Rotation: $[\alpha]_{\mathrm{D}}^{20}=-42.6^{\circ}\left(c=1.0, \mathrm{CHCl}_{3}\right)$.

Physical properties: pale yellow oil; Yield: $44 \%, 15.3 \mathrm{mg}$; Reaction time: $74.7 \mathrm{~h}$.

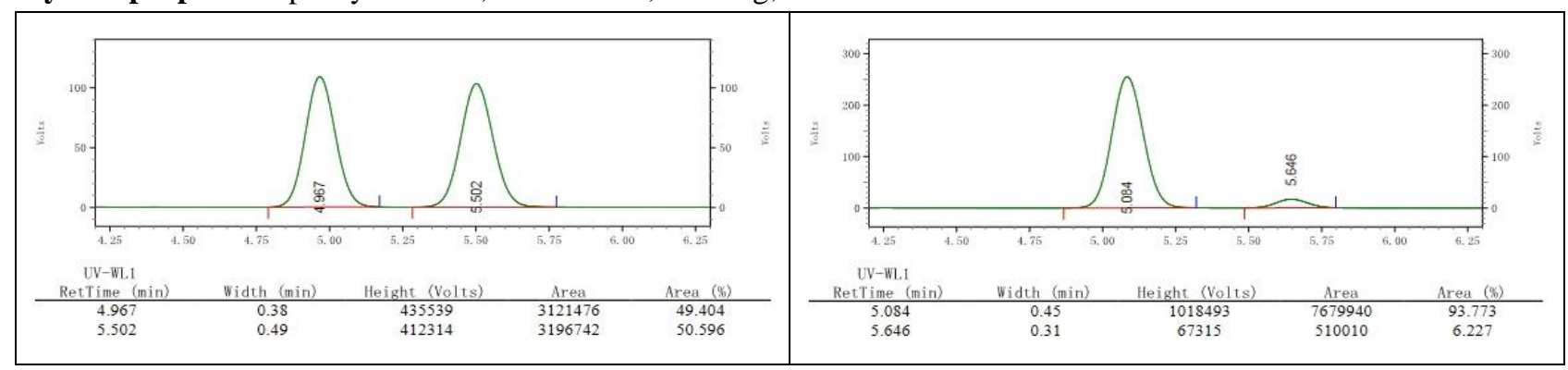

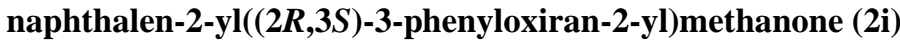

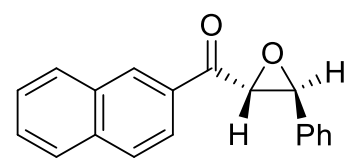

$(2 R, 3 S)-2 \mathbf{i}$

${ }^{\mathbf{1}} \mathbf{H}$ NMR $\left(400 \mathrm{MHz}, \mathrm{CDCl}_{3}\right): \delta 8.55(\mathrm{~s}, 1 \mathrm{H}), 8.04(\mathrm{~d}, J=8.5 \mathrm{~Hz}, 1 \mathrm{H}), 7.95-7.85(\mathrm{~m}, 3 \mathrm{H})$, $7.62(\mathrm{t}, J=7.4 \mathrm{~Hz}, 1 \mathrm{H}), 7.55(\mathrm{t}, J=7.4 \mathrm{~Hz}, 1 \mathrm{H}), 7.46-7.35(\mathrm{~m}, 5 \mathrm{H}), 4.44(\mathrm{~d}, J=1.9 \mathrm{~Hz}$ $1 \mathrm{H}), 4.15(\mathrm{~d}, J=1.9 \mathrm{~Hz}, 1 \mathrm{H})$.

${ }^{13}$ C NMR (100 MHz, $\left.\mathrm{CDCl}_{3}\right): \delta 192.9,135.8,135.5,132.8,132.3,130.4,129.6,129.0,129.0$, $128.8,128.7,127.8,127.0,125.8,123.6,61.0,59.5$.

HRMS (ESI) m/z Calcd for $\left[\mathrm{C}_{19} \mathrm{H}_{14} \mathrm{NaO}_{2}, \mathrm{M}+\mathrm{Na}\right]^{+}:$297.0886, Found: 297.0891.

HPLC analysis: Chiralcel AD-H $($ Hexane $/ i-\mathrm{PrOH}=90: 10$, flow rate $=1.0 \mathrm{~mL} / \mathrm{min}$, wave length $=210 \mathrm{~nm}), t_{\mathrm{R}}=14.453$ $\min$ (minor), $t_{\mathrm{R}}=16.087$ min (major).

Optical Rotation: $[\alpha]_{\mathrm{D}}^{20}=-62.0^{\circ}\left(c=1.0, \mathrm{CHCl}_{3}\right)$.

Physical properties: white solid; Melting Point: $120.5-121.6^{\circ} \mathrm{C}$; Yield: $48 \%, 13.2 \mathrm{mg}$; Reaction time: $74.7 \mathrm{~h}$.

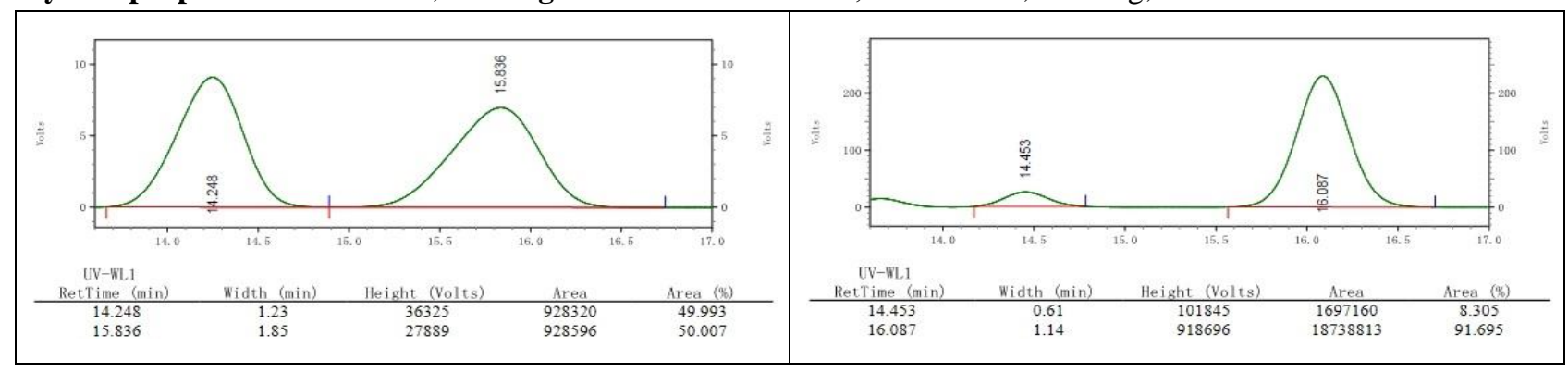


<smiles>CC(C)(C)OOC(CC(=O)c1ccco1)c1ccccc1</smiles>

$(S)-\mathbf{1 j}$

${ }^{1} \mathbf{H}$ NMR $\left(400 \mathrm{MHz}, \mathrm{CDCl}_{3}\right): \delta 7.58(\mathrm{~d}, J=1.8 \mathrm{~Hz}, 1 \mathrm{H}), 7.42(\mathrm{~d}, J=7.1 \mathrm{~Hz}, 2 \mathrm{H}), 7.35(\mathrm{t}, J=$ $7.2 \mathrm{~Hz}, 2 \mathrm{H}), 7.30(\mathrm{~d}, J=7.0 \mathrm{~Hz}, 1 \mathrm{H}), 7.20(\mathrm{~d}, J=3.6 \mathrm{~Hz}, 1 \mathrm{H}), 6.52(\mathrm{dd}, J=3.6,1.8 \mathrm{~Hz}, 1 \mathrm{H})$, $5.62-5.50(\mathrm{~m}, 1 \mathrm{H}), 3.60(\mathrm{dd}, J=15.5,7.9 \mathrm{~Hz}, 1 \mathrm{H}), 3.04(\mathrm{dd}, J=15.6,5.3 \mathrm{~Hz}, 1 \mathrm{H}), 1.16(\mathrm{~s}$, $9 \mathrm{H})$.

${ }^{13}$ C NMR $\left(100 \mathrm{MHz}, \mathrm{CDCl}_{3}\right): \delta 186.1,152.7,146.4,139.7,128.3,128.1,126.9,117.4,112.2,82.0,80.7,44.1,26.2$.

HRMS (ESI) $\mathrm{m} / \mathrm{z}$ Calcd for $\left[\mathrm{C}_{17} \mathrm{H}_{20} \mathrm{NaO}_{4}, \mathrm{M}+\mathrm{Na}\right]^{+}: 311.1254$, Found: 311.1257.

HPLC analysis: Chiralcel IA-H (Hexane $/ i-\mathrm{PrOH}=95: 5$, flow rate $=1.0 \mathrm{~mL} / \mathrm{min}$, wave length $=254 \mathrm{~nm}), t_{\mathrm{R}}=5.448 \mathrm{~min}$ (major), $t_{\mathrm{R}}=5.813 \mathrm{~min}$ (minor).

Optical Rotation: $[\alpha]_{\mathrm{D}}^{20}=-42 \cdot 6^{\circ}\left(c=1.0, \mathrm{CHCl}_{3}\right)$.

Physical properties: white solid; Melting Point: $59.1-60.3^{\circ} \mathrm{C}$; Yield: $45 \%, 13.0 \mathrm{mg}$; Reaction time: $35.5 \mathrm{~h}$.

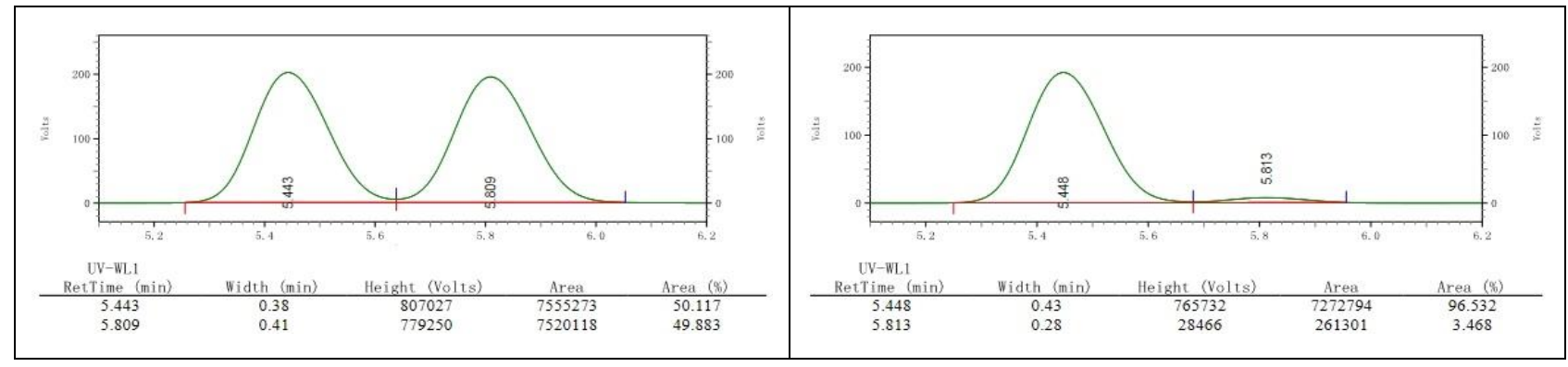

furan-2-yl((2R,3S)-3-phenyloxiran-2-yl)methanone (2j)

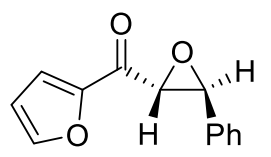

$(2 R, 3 S)-2 \mathrm{j}$

${ }^{1} \mathrm{H}$ NMR $\left(400 \mathrm{MHz}, \mathrm{CDCl}_{3}\right): \delta 7.67(\mathrm{~d}, J=1.7 \mathrm{~Hz}, 1 \mathrm{H}), 7.45(\mathrm{~d}, J=3.5 \mathrm{~Hz}, 1 \mathrm{H}), 7.39-7.32(\mathrm{~m}, 5 \mathrm{H})$, $6.59(\mathrm{dd}, J=3.5,1.6 \mathrm{~Hz}, 1 \mathrm{H}), 4.14(\mathrm{~s}, 2 \mathrm{H})$.

${ }^{13}$ C NMR $\left(100 \mathrm{MHz}, \mathrm{CDCl}_{3}\right): \delta 181.9,151.0,147.6,135.2,128.9,128.6,125.7,119.5,112.6,60.5$, 59.5 .

HRMS (ESI) $\mathrm{m} / \mathrm{z}$ Calcd for $\left[\mathrm{C}_{13} \mathrm{H}_{10} \mathrm{NaO}_{3}, \mathrm{M}+\mathrm{Na}\right]^{+}: 237.0522$, Found: 237.0518.

HPLC analysis: Chiralcel IA-H (Hexane $/ i-\mathrm{PrOH}=90: 10$, flow rate $=1.0 \mathrm{~mL} / \mathrm{min}$, wave length $=254 \mathrm{~nm}), t_{\mathrm{R}}=11.192$ $\min$ (minor), $t_{\mathrm{R}}=11.798 \mathrm{~min}$ (major).

Optical Rotation: $[\alpha]_{\mathrm{D}}^{20}=-97.8^{\circ}\left(c=1.0, \mathrm{CHCl}_{3}\right)$.

Physical properties: pale yellow solid; Melting Point: $61.6-62.1^{\circ} \mathrm{C}$; Yield: 50\%, $10.7 \mathrm{mg}$; Reaction time: $35.5 \mathrm{~h}$.

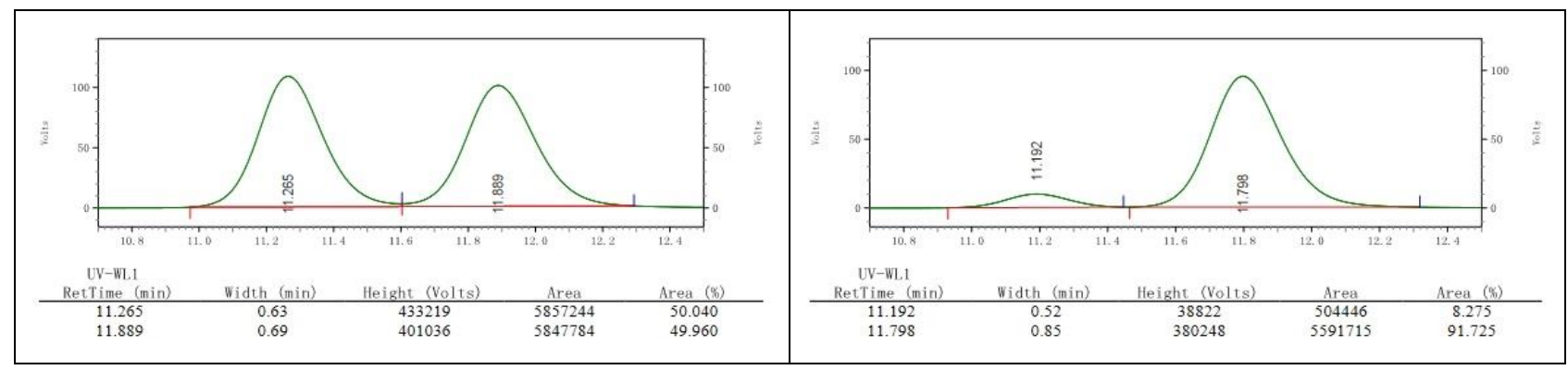


<smiles>CC(C)(C)OOC(CC(=O)c1cccs1)c1ccccc1</smiles>

$(S)-1 \mathbf{k}$

${ }^{1} \mathrm{H}$ NMR $\left(400 \mathrm{MHz}, \mathrm{CDCl}_{3}\right): \delta 7.72(\mathrm{~d}, J=3.7 \mathrm{~Hz}, 1 \mathrm{H}), 7.61(\mathrm{~d}, J=4.9 \mathrm{~Hz}, 1 \mathrm{H}), 7.42(\mathrm{~d}, J=$ $7.2 \mathrm{~Hz}, 2 \mathrm{H}), 7.34(\mathrm{t}, J=7.3 \mathrm{~Hz}, 2 \mathrm{H}), 7.31-7.26(\mathrm{~m}, 1 \mathrm{H}), 7.10(\mathrm{dd}, J=4.9,3.8 \mathrm{~Hz}, 1 \mathrm{H}), 5.57$ $(\mathrm{dd}, J=7.5,5.6 \mathrm{~Hz}, 1 \mathrm{H}), 3.66(\mathrm{dd}, J=15.6,7.5 \mathrm{~Hz}, 1 \mathrm{H}), 3.13(\mathrm{dd}, J=15.6,5.6 \mathrm{~Hz}, 1 \mathrm{H}), 1.16$ (s, 9H).

${ }^{13}$ C NMR $\left(100 \mathrm{MHz}, \mathrm{CDCl}_{3}\right): \delta$ 190.0, 144.5, 139.7, 133.8, 132.2, 128.4, 128.1, 128.0, 126.9, 82.2, 80.8, 44.9, 26.3.

HRMS (ESI) $\mathrm{m} / \mathrm{z}$ Calcd for $\left[\mathrm{C}_{17} \mathrm{H}_{20} \mathrm{NaO}_{3} \mathrm{~S}, \mathrm{M}+\mathrm{Na}\right]^{+}:$327.1025, Found: 327.1024.

HPLC analysis: Chiralcel IA-H (Hexane $/ i-\mathrm{PrOH}=97: 3$, flow rate $=1.0 \mathrm{~mL} / \mathrm{min}$, wave length $=254 \mathrm{~nm}), t_{\mathrm{R}}=6.188 \mathrm{~min}$ (major), $t_{\mathrm{R}}=6.630 \mathrm{~min}$ (minor).

Optical Rotation: $[\alpha]_{\mathrm{D}}^{20}=-34.9^{\circ}\left(c=1.0, \mathrm{CHCl}_{3}\right)$.

Physical properties: white solid; Melting Point: $56.8-57.8^{\circ} \mathrm{C}$; Yield: $46 \%, 14.0 \mathrm{mg}$; Reaction time: $47.0 \mathrm{~h}$.

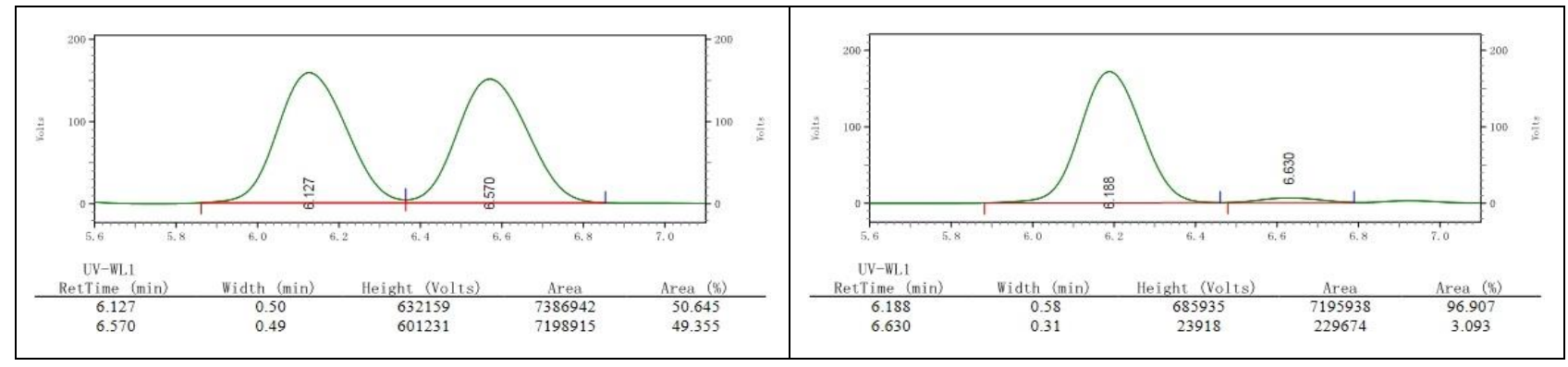

$((2 R, 3 S)$-3-phenyloxiran-2-yl)(thiophen-2-yl)methanone (2k)<smiles>O=C(COc1ccccc1)c1cccs1</smiles>

$(2 R, 3 S)-\mathbf{2 k}$

${ }^{1} \mathbf{H}$ NMR $\left(400 \mathrm{MHz}, \mathrm{CDCl}_{3}\right): \delta 7.99(\mathrm{~d}, J=3.7 \mathrm{~Hz}, 1 \mathrm{H}), 7.74(\mathrm{~d}, J=4.9 \mathrm{~Hz}, 1 \mathrm{H}), 7.40-7.32(\mathrm{~m}, 5 \mathrm{H})$, $7.17(\mathrm{t}, J=4.4 \mathrm{~Hz}, 1 \mathrm{H}), 4.16(\mathrm{~d}, J=1.4 \mathrm{~Hz}, 1 \mathrm{H}), 4.08(\mathrm{~d}, J=1.7 \mathrm{~Hz}, 1 \mathrm{H})$.

${ }^{13} \mathbf{C}$ NMR (100 MHz, $\left.\mathrm{CDCl}_{3}\right): \delta 186.4,140.9,135.2,133.6,129.0,128.7,128.4,125.7,61.9,59.4$.

HRMS (ESI) $\mathrm{m} / \mathrm{z}$ Calcd for $\left[\mathrm{C}_{13} \mathrm{H}_{10} \mathrm{NaO}_{2} \mathrm{~S}, \mathrm{M}+\mathrm{Na}\right]^{+}:$253.0294, Found: 253.0290 .

HPLC analysis: Chiralcel IA-H (Hexane $/ i-\mathrm{PrOH}=85: 15$, flow rate $=1.0 \mathrm{~mL} / \mathrm{min}$, wave length $=254 \mathrm{~nm}), t_{\mathrm{R}}=8.963 \mathrm{~min}$ (major), $t_{\mathrm{R}}=9.589 \mathrm{~min}$ (minor).

Optical Rotation: $[\alpha]_{\mathrm{D}}^{20}=-122 \cdot 6^{\circ}\left(c=1.0, \mathrm{CHCl}_{3}\right)$.

Physical properties: pale yellow solid; Melting Point: $58.4-59.3{ }^{\circ} \mathrm{C}$; Yield: $51 \%, 11.7 \mathrm{mg}$; Reaction time: $47.0 \mathrm{~h}$.

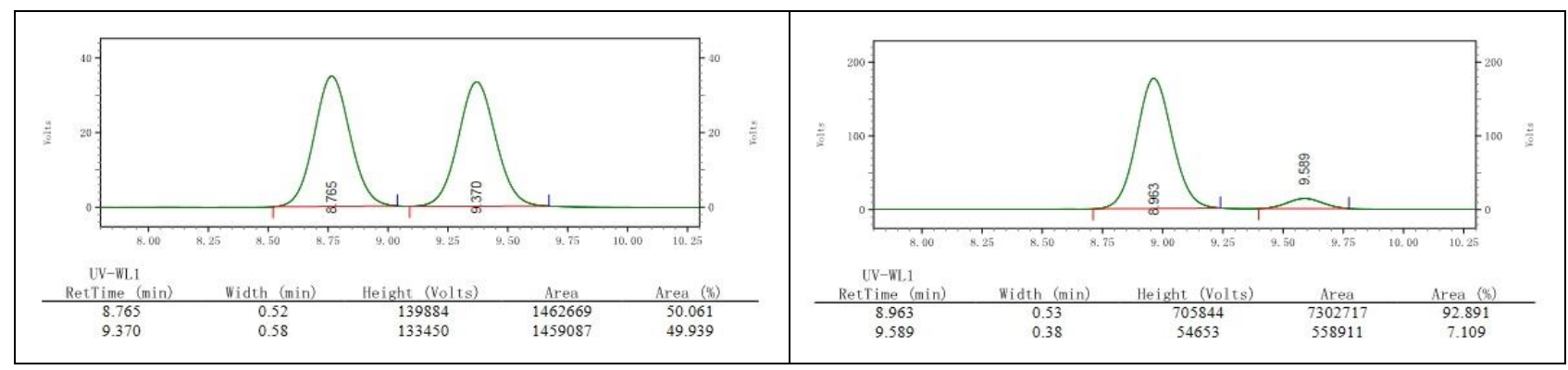


<smiles>Cc1ccc(C(CC(=O)c2ccccc2)OOC(C)(C)C)cc1</smiles>

(S)-11

${ }^{1} \mathbf{H}$ NMR (400 MHz, $\left.\mathrm{CDCl}_{3}\right): \delta 7.94(\mathrm{~d}, J=7.3 \mathrm{~Hz}, 2 \mathrm{H}), 7.51(\mathrm{t}, J=7.4 \mathrm{~Hz}, 1 \mathrm{H}), 7.42(\mathrm{t}, J=$ $7.6 \mathrm{~Hz}, 2 \mathrm{H}), 7.32(\mathrm{~d}, J=7.9 \mathrm{~Hz}, 2 \mathrm{H}), 7.14(\mathrm{~d}, J=7.8 \mathrm{~Hz}, 2 \mathrm{H}), 5.57$ (t, $J=6.5 \mathrm{~Hz}, 1 \mathrm{H}), 3.78$ $(\mathrm{dd}, J=16.2,7.0 \mathrm{~Hz}, 1 \mathrm{H}), 3.20(\mathrm{dd}, J=16.2,6.0 \mathrm{~Hz}, 1 \mathrm{H}), 2.31(\mathrm{~s}, 3 \mathrm{H}), 1.16(\mathrm{~s}, 9 \mathrm{H})$.

${ }^{13} \mathrm{C}$ NMR $\left(100 \mathrm{MHz}, \mathrm{CDCl}_{3}\right): \delta 197.4,137.7,137.1,136.8,132.9,129.0,128.4,128.1,127.0$, $82.2,80.6,43.8,26.3,21.1$.

HRMS (ESI) m/z Calcd for $\left[\mathrm{C}_{20} \mathrm{H}_{24} \mathrm{NaO}_{3}, \mathrm{M}+\mathrm{Na}\right]^{+}: 335.1618$, Found: 335.1624

HPLC analysis: Chiralcel IA-H $($ Hexane $/ i-\mathrm{PrOH}=90: 10$, flow rate $=1.0 \mathrm{~mL} / \mathrm{min}$, wave length $=210 \mathrm{~nm}), t_{\mathrm{R}}=4.368 \mathrm{~min}$ (major), $t_{\mathrm{R}}=4.592 \mathrm{~min}$ (minor).

Optical Rotation: $[\alpha]_{\mathrm{D}}^{20}=-40.5^{\circ}\left(c=1.0, \mathrm{CHCl}_{3}\right)$.

Physical properties: pale yellow solid; Melting Point: $57.1-58.9^{\circ} \mathrm{C}$; Yield: $43 \%, 13.4 \mathrm{mg}$; Reaction time: 112.5 h.

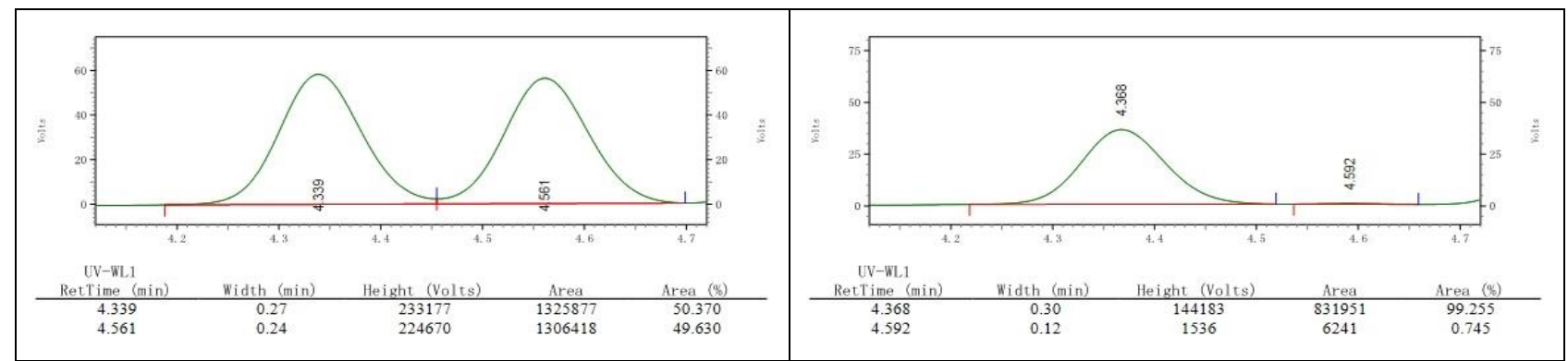

phenyl((2R,3S)-3-(p-tolyl)oxiran-2-yl)methanone (2l)<smiles></smiles>

${ }^{1} \mathbf{H}$ NMR $\left(400 \mathrm{MHz}, \mathrm{CDCl}_{3}\right): \delta 7.99(\mathrm{~d}, J=7.3 \mathrm{~Hz}, 2 \mathrm{H}), 7.59(\mathrm{t}, J=7.4 \mathrm{~Hz}, 1 \mathrm{H}), 7.46(\mathrm{t}, J=7.7 \mathrm{~Hz}$, $2 \mathrm{H}), 7.25(\mathrm{~d}, J=8.0 \mathrm{~Hz}, 2 \mathrm{H}), 7.20(\mathrm{~d}, J=8.0 \mathrm{~Hz}, 2 \mathrm{H}), 4.29(\mathrm{~d}, J=1.7 \mathrm{~Hz}, 1 \mathrm{H}), 4.02(\mathrm{~d}, J=1.9 \mathrm{~Hz}$, $1 \mathrm{H}), 2.36(\mathrm{~s}, 3 \mathrm{H})$.

${ }^{13}$ C NMR (100 MHz, $\left.\mathrm{CDCl}_{3}\right): \delta 193.0,138.9,135.3,133.8,132.3,129.3,128.7,128.1,125.6,60.9$, $59.3,21.1$.

HRMS (ESI) m/z Calcd for $\left[\mathrm{C}_{16} \mathrm{H}_{14} \mathrm{NaO}_{2}, \mathrm{M}+\mathrm{Na}\right]^{+}: 261.0886$, Found: 261.0883

HPLC analysis: Chiralcel IA-H $(\mathrm{Hexane} / i-\mathrm{PrOH}=95: 5$, flow rate $=1.0 \mathrm{~mL} / \mathrm{min}$, wave length $=254 \mathrm{~nm}), t_{\mathrm{R}}=11.658 \mathrm{~min}$ (minor), $t_{\mathrm{R}}=12.727 \mathrm{~min}$ (major).

Optical Rotation: $[\alpha]_{\mathrm{D}}^{20}=-77.5^{\circ}\left(c=1.0, \mathrm{CHCl}_{3}\right)$.

Physical properties: pale yellow solid; Melting Point: $74.3-75.3{ }^{\circ} \mathrm{C}$; Yield: $51 \%, 12.2 \mathrm{mg}$; Reaction time: $112.5 \mathrm{~h}$.

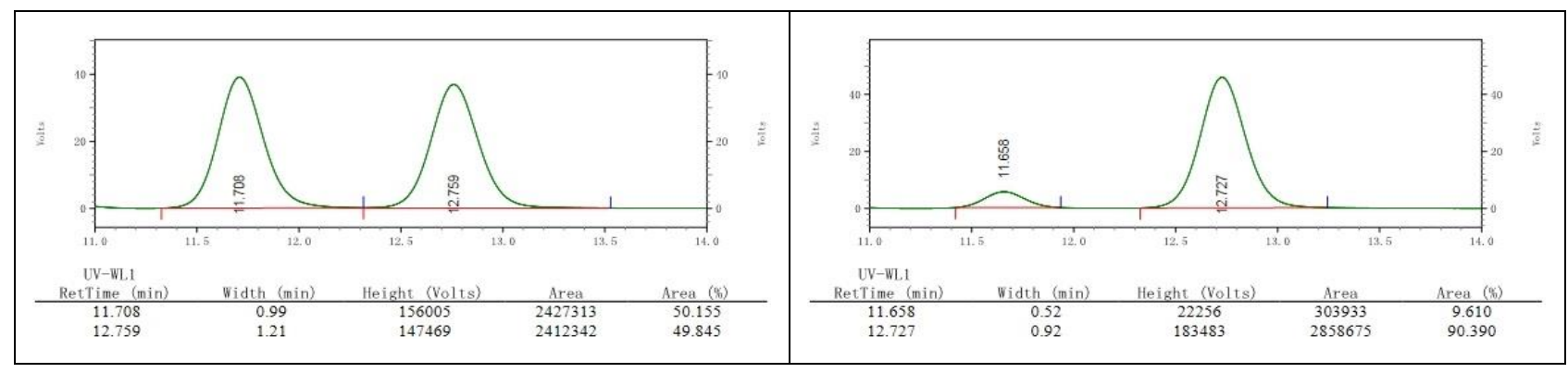


<smiles>CC(C)(C)OOC(CC(=O)c1ccccc1)c1ccc(C(C)(C)C)cc1</smiles>

(S)-1m

${ }^{1} \mathbf{H}$ NMR (400 MHz, $\left.\mathrm{CDCl}_{3}\right): \delta 7.93(\mathrm{~d}, J=7.5 \mathrm{~Hz}, 2 \mathrm{H}), 7.48(\mathrm{t}, J=7.4 \mathrm{~Hz}, 1 \mathrm{H}), 7.39(\mathrm{~d}, J=$ $7.8 \mathrm{~Hz}, 2 \mathrm{H}), 7.37-7.31(\mathrm{~m}, 4 \mathrm{H}), 5.61(\mathrm{t}, J=6.4 \mathrm{~Hz}, 1 \mathrm{H}), 3.78(\mathrm{dd}, J=16.2,7.0 \mathrm{~Hz}, 1 \mathrm{H}), 3.19$ (dd, $J=16.2,5.8 \mathrm{~Hz}, 1 \mathrm{H}), 1.29(\mathrm{~s}, 9 \mathrm{H}), 1.16(\mathrm{~s}, 9 \mathrm{H})$.

${ }^{13} \mathrm{C}$ NMR $\left(100 \mathrm{MHz}, \mathrm{CDCl}_{3}\right): \delta 197.3,150.7,137.0,136.7,132.8,128.3,128.1,126.7,125.1$, $82.0,80.5,43.9,34.3,31.2,26.2$.

HRMS (ESI) m/z Calcd for $\left[\mathrm{C}_{23} \mathrm{H}_{30} \mathrm{NaO}_{3}, \mathrm{M}+\mathrm{Na}\right]^{+}:$377.2087, Found: 377.2081.

HPLC analysis: Chiralcel IA-H $($ Hexane $/ i-\mathrm{PrOH}=98: 2$, flow rate $=1.0 \mathrm{~mL} / \mathrm{min}$, wave length $=254 \mathrm{~nm}), t_{\mathrm{R}}=4.744 \mathrm{~min}$ (major), $t_{\mathrm{R}}=5.376 \mathrm{~min}$ (minor).

Optical Rotation: $[\alpha]_{\mathrm{D}}^{20}=-40.1^{\circ}\left(c=1.0, \mathrm{CHCl}_{3}\right)$.

Physical properties: pale yellow oil; Yield: 44\%, $15.6 \mathrm{mg}$; Reaction time: $137.0 \mathrm{~h}$.

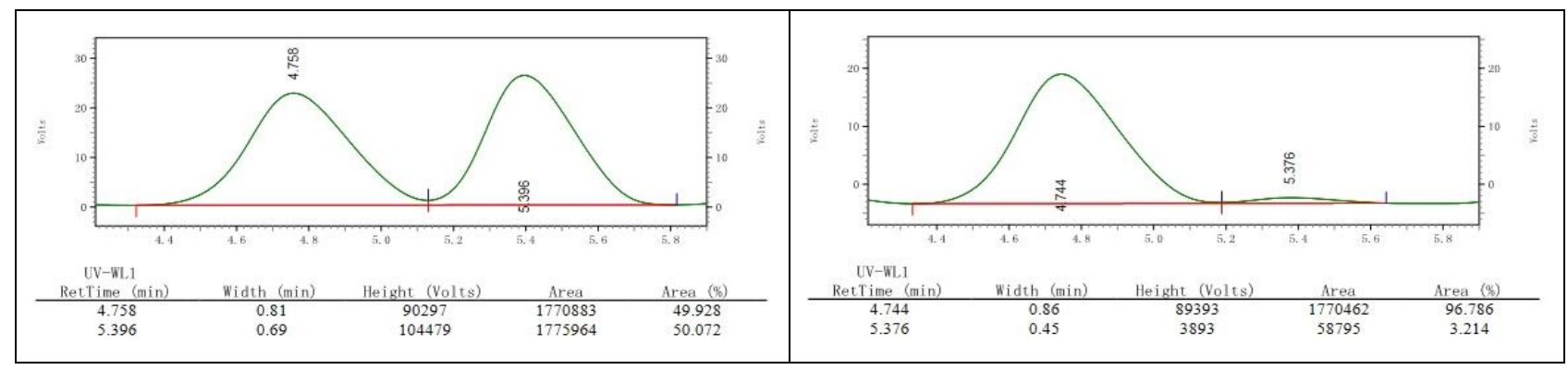

((2R,3S)-3-(4-(tert-butyl)phenyl)oxiran-2-yl)(phenyl)methanone (2m)

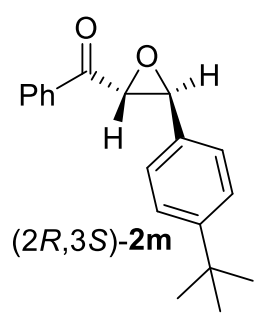

${ }^{1} \mathbf{H}$ NMR $\left(400 \mathrm{MHz}, \mathrm{CDCl}_{3}\right): \delta 7.99(\mathrm{~d}, J=7.7 \mathrm{~Hz}, 2 \mathrm{H}), 7.58(\mathrm{t}, J=7.3 \mathrm{~Hz}, 1 \mathrm{H}), 7.47-7.40(\mathrm{~m}, 4 \mathrm{H})$, $7.30(\mathrm{~d}, J=8.1 \mathrm{~Hz}, 2 \mathrm{H}), 4.31(\mathrm{~d}, J=1.9 \mathrm{~Hz}, 1 \mathrm{H}), 4.04(\mathrm{~d}, J=2.1 \mathrm{~Hz}, 1 \mathrm{H}), 1.32(\mathrm{~s}, 9 \mathrm{H})$.

${ }^{13}$ C NMR (100 MHz, $\left.\mathrm{CDCl}_{3}\right): \delta 193.0,152.1,135.3,133.8,132.3,128.7,128.2,125.5,125.5,60.8$, $59.2,34.5,31.1$.

HRMS (ESI) m/z Calcd for $\left[\mathrm{C}_{19} \mathrm{H}_{20} \mathrm{NaO}_{2}, \mathrm{M}+\mathrm{Na}\right]^{+}:$303.1356, Found: 303.1361.

HPLC analysis: Chiralcel OJ-H $(\mathrm{Hexane} / i-\mathrm{PrOH}=80: 20$, flow rate $=1.0 \mathrm{~mL} / \mathrm{min}$, wave length $=254$ $\mathrm{nm}), t_{\mathrm{R}}=6.868 \min ($ minor $), t_{\mathrm{R}}=9.262 \min$ (major).

Optical Rotation: $[\alpha]_{\mathrm{D}}^{20}=-115.9^{\circ}\left(c=1.0, \mathrm{CHCl}_{3}\right)$.

Physical properties: white solid; Melting Point: $79.1-80.4{ }^{\circ} \mathrm{C}$; Yield: 49\%, $13.7 \mathrm{mg}$; Reaction time: $137.0 \mathrm{~h}$.

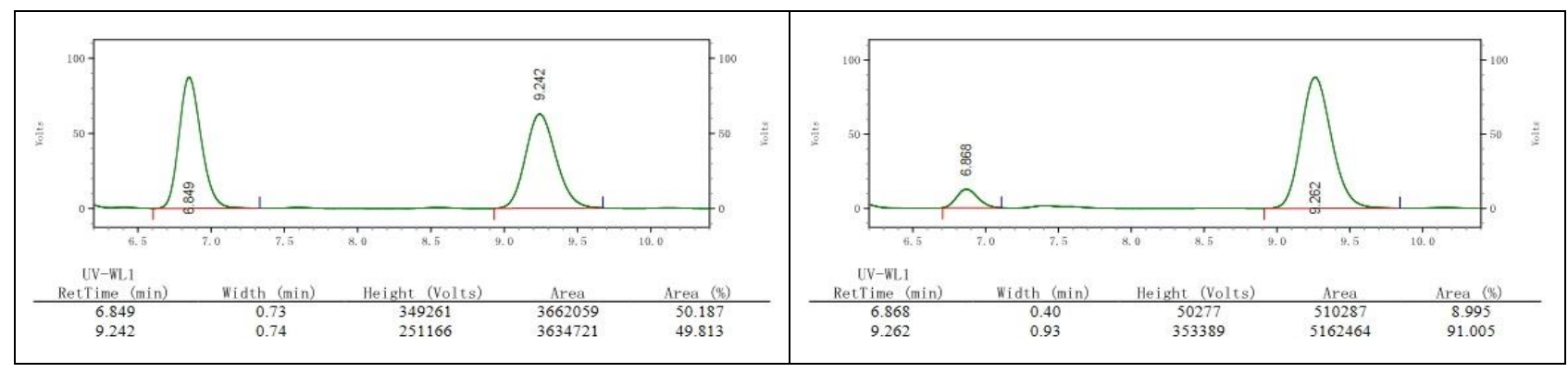


<smiles>CC(C)(C)OOC(CC(=O)c1ccccc1)c1ccc(F)cc1</smiles>

$(S)-1 n$

${ }^{1} \mathbf{H}$ NMR $\left(400 \mathrm{MHz}, \mathrm{CDCl}_{3}\right): \delta 7.94(\mathrm{~d}, J=7.5 \mathrm{~Hz}, 2 \mathrm{H}), 7.52(\mathrm{t}, J=7.3 \mathrm{~Hz}, 1 \mathrm{H}), 7.47-7.34(\mathrm{~m}$, $4 \mathrm{H}), 7.01(\mathrm{t}, J=8.6 \mathrm{~Hz}, 2 \mathrm{H}), 5.59(\mathrm{t}, J=6.5 \mathrm{~Hz}, 1 \mathrm{H}), 3.75(\mathrm{dd}, J=16.4,6.9 \mathrm{~Hz}, 1 \mathrm{H}), 3.19(\mathrm{dd}, J$ $=16.4,6.2 \mathrm{~Hz}, 1 \mathrm{H}), 1.16(\mathrm{~s}, 9 \mathrm{H})$.

${ }^{13} \mathbf{C}$ NMR $\left(100 \mathrm{MHz}, \mathrm{CDCl}_{3}\right): \delta 196.9,162.3(\mathrm{~d}, J=244.8 \mathrm{~Hz}), 136.9,135.8(\mathrm{~d}, J=2.9 \mathrm{~Hz}), 133.0$, $128.7(\mathrm{~d}, J=8.1 \mathrm{~Hz}), 128.4,128.0,115.1(\mathrm{~d}, J=21.3 \mathrm{~Hz}), 81.4,80.6,43.7,26.2$.

HRMS (ESI) m/z Calcd for $\left[\mathrm{C}_{19} \mathrm{H}_{21} \mathrm{FNaO}_{3}, \mathrm{M}+\mathrm{Na}\right]^{+}: 339.1367$, Found: 339.1375.

HPLC analysis: Chiralcel IA-H (Hexane $/ i-\mathrm{PrOH}=95: 5$, flow rate $=1.0 \mathrm{~mL} / \mathrm{min}$, wave length $=254 \mathrm{~nm}), t_{\mathrm{R}}=5.341 \mathrm{~min}$ (major), $t_{\mathrm{R}}=5.733 \mathrm{~min}$ (minor).

Optical Rotation: $[\alpha]_{\mathrm{D}}^{20}=-53.7^{\circ}\left(c=1.0, \mathrm{CHCl}_{3}\right)$.

Physical properties: pale yellow solid; Melting Point: $52.1-53.4^{\circ} \mathrm{C}$; Yield: $43 \%, 13.6 \mathrm{mg}$; Reaction time: $108.5 \mathrm{~h}$.

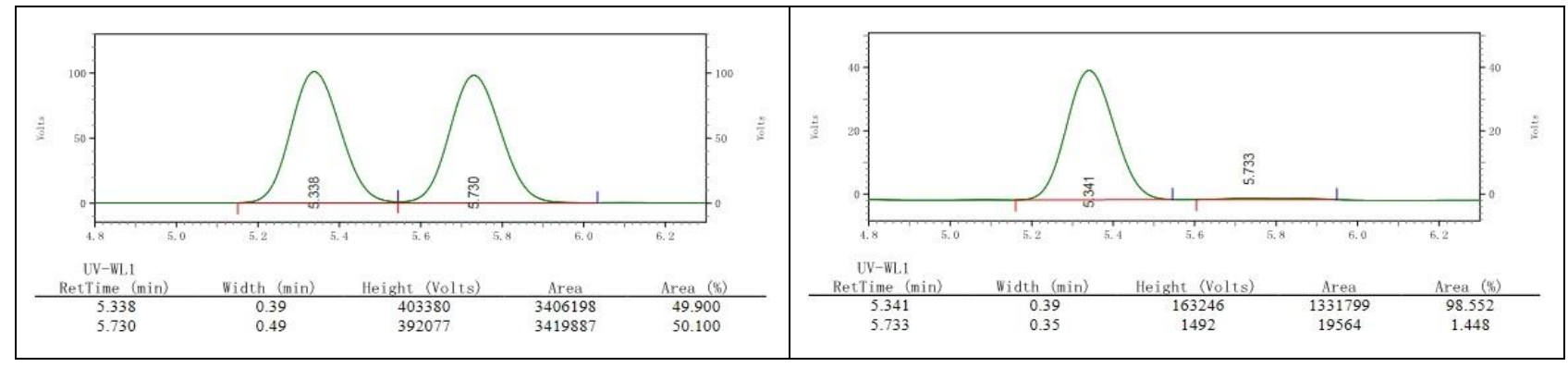

((2R,3S)-3-(4-fluorophenyl)oxiran-2-yl)(phenyl)methanone (2n)

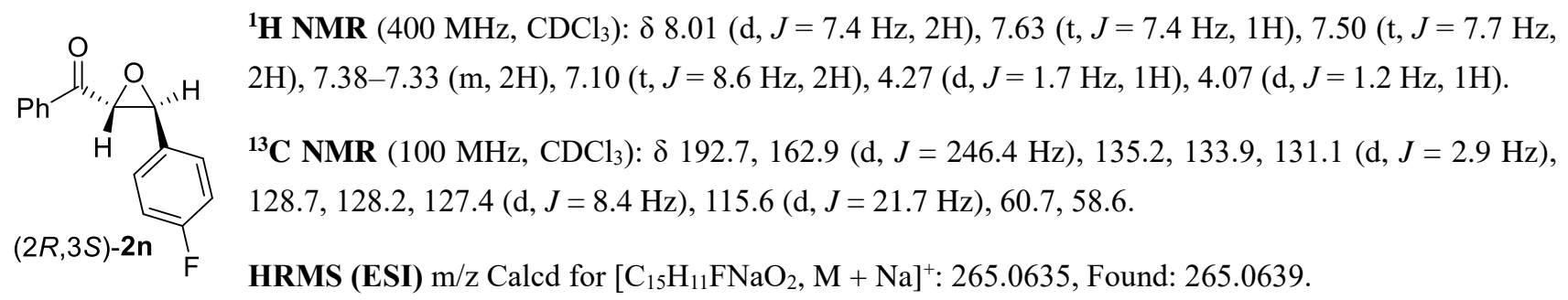

HPLC analysis: Chiralcel IA-H (Hexane $/ i-\mathrm{PrOH}=95: 5$, flow rate $=1.0 \mathrm{~mL} / \mathrm{min}$, wave length $=254 \mathrm{~nm}), t_{\mathrm{R}}=13.488 \mathrm{~min}$ (minor), $t_{\mathrm{R}}=14.208 \mathrm{~min}$ (major).

Optical Rotation: $[\alpha]_{\mathrm{D}}^{20}=-114.7^{\circ}\left(c=1.0, \mathrm{CHCl}_{3}\right)$.

Physical properties: white solid; Melting Point: $89.5-90.0^{\circ} \mathrm{C}$; Yield: $51 \%, 12.4 \mathrm{mg}$; Reaction time: $108.5 \mathrm{~h}$.

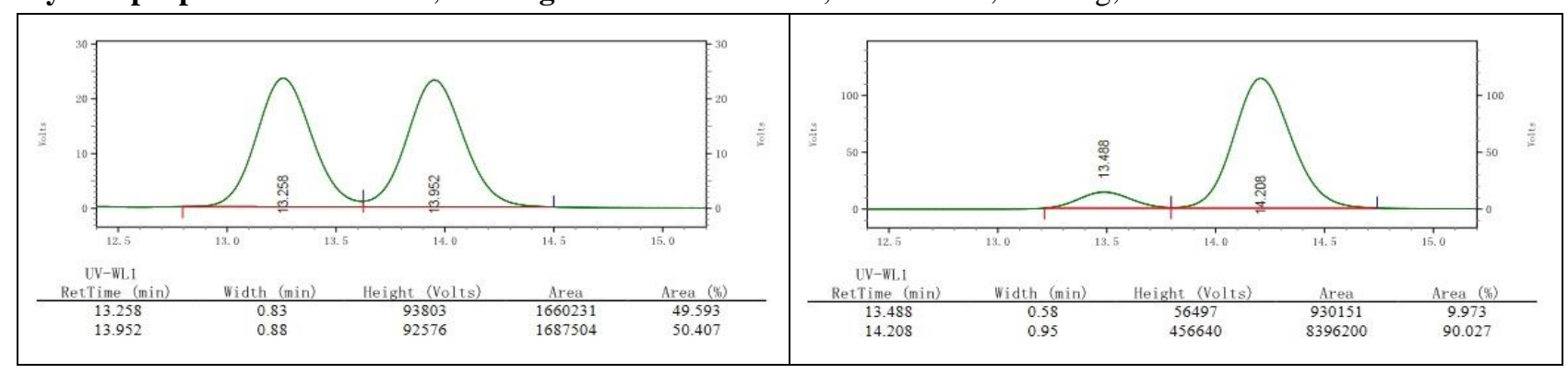


(S)-3-(tert-butylperoxy)-3-(4-chlorophenyl)-1-phenylpropan-1-one (10)<smiles>CC(C)(C)OO[C@H](CC(=O)c1ccccc1)c1ccc(Cl)cc1</smiles>

$(S)-10$

${ }^{1} \mathbf{H}$ NMR (400 MHz, $\left.\mathrm{CDCl}_{3}\right): \delta 7.94(\mathrm{~d}, J=7.3 \mathrm{~Hz}, 2 \mathrm{H}), 7.56(\mathrm{t}, J=7.4 \mathrm{~Hz}, 1 \mathrm{H}), 7.45(\mathrm{t}, J=7.6$ $\mathrm{Hz}, 2 \mathrm{H}), 7.37$ (d, $J=8.4 \mathrm{~Hz}, 2 \mathrm{H}), 7.32$ (d, $J=8.5 \mathrm{~Hz}, 2 \mathrm{H}), 5.56(\mathrm{t}, J=6.5 \mathrm{~Hz}, 1 \mathrm{H}), 3.73$ (dd, $J=$ $16.4,6.9 \mathrm{~Hz}, 1 \mathrm{H}), 3.18$ (dd, $J=16.4,6.2 \mathrm{~Hz}, 1 \mathrm{H}), 1.16$ (s, 9H).

${ }^{13} \mathbf{C}$ NMR $\left(100 \mathrm{MHz}, \mathrm{CDCl}_{3}\right): \delta 197.0,138.7,136.9,133.8,133.2,128.6,128.5,128.2,81.5,80.9$, 43.7, 26.3.

HRMS (ESI) m/z Calcd for $\left[\mathrm{C}_{19} \mathrm{H}_{21} \mathrm{ClNaO}_{3}, \mathrm{M}+\mathrm{Na}\right]^{+}:$355.1071, Found: 355.1077.

HPLC analysis: Chiralcel IA-H $($ Hexane $/ i-\mathrm{PrOH}=97: 3$, flow rate $=1.0 \mathrm{~mL} / \mathrm{min}$, wave length $=254 \mathrm{~nm}), t_{\mathrm{R}}=5.973 \mathrm{~min}$ (major), $t_{\mathrm{R}}=6.598 \mathrm{~min}$ (minor).

Optical Rotation: $[\alpha]_{\mathrm{D}}^{20}=-32.7^{\circ}\left(c=1.0, \mathrm{CHCl}_{3}\right)$.

Physical properties: white solid; Melting Point: $64.3-65.4{ }^{\circ} \mathrm{C}$; Yield: $45 \%, 15.0 \mathrm{mg}$; Reaction time: $87.5 \mathrm{~h}$.

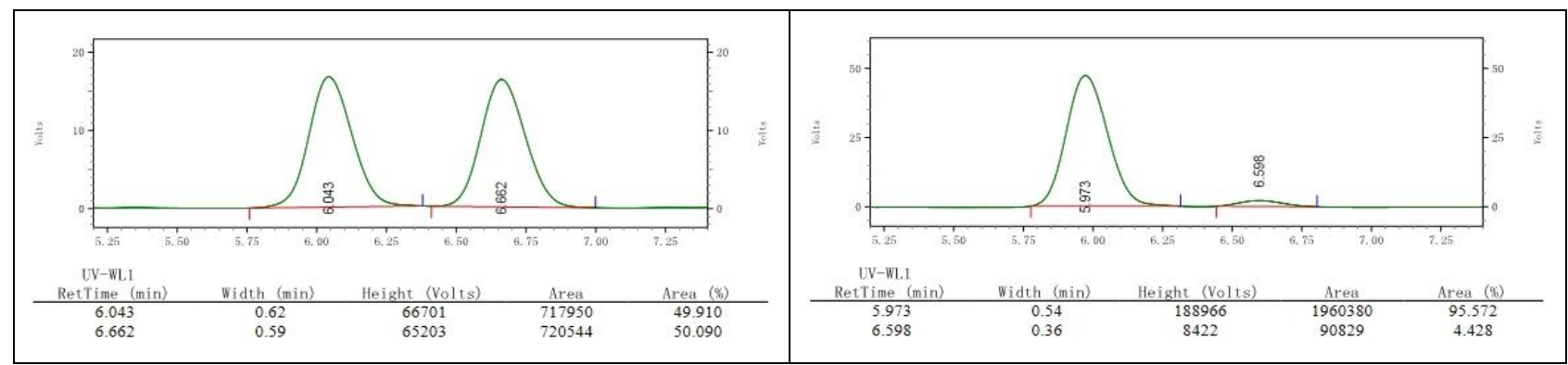

((2R,3S)-3-(4-chlorophenyl)oxiran-2-yl)(phenyl)methanone (2o)<smiles>[R16][R6]c1ccc([C@@H]2O[C@H]2C(=O)c2ccccc2)cc1</smiles>

${ }^{1} \mathbf{H}$ NMR $\left(400 \mathrm{MHz}, \mathrm{CDCl}_{3}\right): \delta 7.98(\mathrm{~d}, J=7.6 \mathrm{~Hz}, 2 \mathrm{H}), 7.60(\mathrm{t}, J=7.4 \mathrm{~Hz}, 1 \mathrm{H}), 7.47(\mathrm{t}, J=7.7 \mathrm{~Hz}$, 2H), $7.35(\mathrm{~d}, J=8.5 \mathrm{~Hz}, 2 \mathrm{H}), 7.29(\mathrm{~d}, J=8.5 \mathrm{~Hz}, 2 \mathrm{H}), 4.26(\mathrm{~d}, J=1.7 \mathrm{~Hz}, 1 \mathrm{H}), 4.04(\mathrm{~d}, J=1.9 \mathrm{~Hz}$, $1 \mathrm{H})$.

${ }^{13}$ C NMR (100 MHz, $\left.\mathrm{CDCl}_{3}\right): \delta 192.5,135.1,134.7,133.9,133.9,128.8,128.7,128.1,127.0,60.7$, 58.5 .

HRMS (ESI) m/z Calcd for $\left[\mathrm{C}_{15} \mathrm{H}_{11} \mathrm{ClNaO}_{2}, \mathrm{M}+\mathrm{Na}\right]^{+}:$281.0340, Found: 281.0345.

HPLC analysis: Chiralcel IA-H $($ Hexane $/ i-\mathrm{PrOH}=95: 5$, flow rate $=1.0 \mathrm{~mL} / \mathrm{min}$, wave length $=254 \mathrm{~nm}), t_{\mathrm{R}}=13.405 \mathrm{~min}$ (minor), $t_{\mathrm{R}}=15.175 \mathrm{~min}$ (major).

Optical Rotation: $[\alpha]_{\mathrm{D}}^{20}=-81.5^{\circ}\left(c=1.0, \mathrm{CHCl}_{3}\right)$.

Physical properties: white solid; Melting Point: $72.9-73.5^{\circ} \mathrm{C}$; Yield: $50 \%, 12.9 \mathrm{mg}$; Reaction time: $87.5 \mathrm{~h}$.

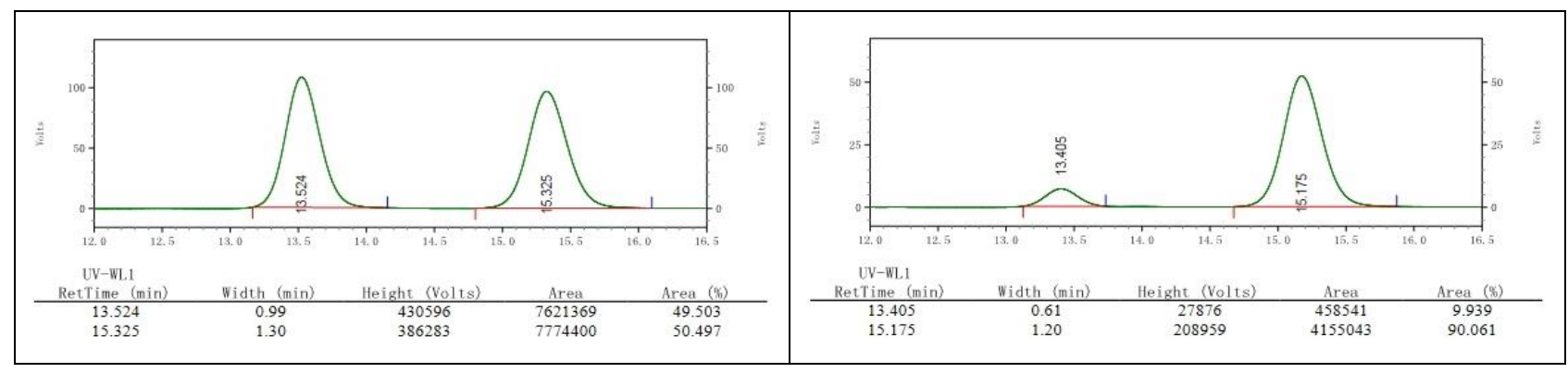


(S)-3-(4-bromophenyl)-3-(tert-butylperoxy)-1-phenylpropan-1-one (1p)<smiles>CC(C)(C)OO[C@H](CC(=O)c1ccccc1)c1ccc(Br)cc1</smiles>

(S)-1p

${ }^{1} \mathbf{H}$ NMR (400 MHz, $\left.\mathrm{CDCl}_{3}\right): \delta 7.94(\mathrm{~d}, J=7.5 \mathrm{~Hz}, 2 \mathrm{H}), 7.56(\mathrm{t}, J=7.3 \mathrm{~Hz}, 1 \mathrm{H}), 7.51-7.40(\mathrm{~m}$, $4 \mathrm{H}), 7.31(\mathrm{~d}, J=8.2 \mathrm{~Hz}, 2 \mathrm{H}), 5.55(\mathrm{t}, J=6.5 \mathrm{~Hz}, 1 \mathrm{H}), 3.72(\mathrm{dd}, J=16.4,6.9 \mathrm{~Hz}, 1 \mathrm{H}), 3.17(\mathrm{dd}, J$ $=16.4,6.1 \mathrm{~Hz}, 1 \mathrm{H}), 1.16(\mathrm{~s}, 9 \mathrm{H})$.

${ }^{13}$ C NMR (100 MHz, $\left.\mathrm{CDCl}_{3}\right): \delta 196.7,139.2,136.8,133.1,131.4,128.7,128.5,128.1,121.8$, $81.4,80.7,43.6,26.2$.

HRMS (ESI) m/z Calcd for $\left[\mathrm{C}_{19} \mathrm{H}_{21} \mathrm{BrNaO}_{3}, \mathrm{M}+\mathrm{Na}\right]^{+}:$399.0566, Found: 399.0575.

HPLC analysis: Chiralcel AD-H (Hexane $/ i-\mathrm{PrOH}=99: 1$, flow rate $=1.0 \mathrm{~mL} / \mathrm{min}$, wave length $=254 \mathrm{~nm}), t_{\mathrm{R}}=14.078$ $\min$ (major), $t_{\mathrm{R}}=18.553 \mathrm{~min}$ (minor).

Optical Rotation: $[\alpha]_{\mathrm{D}}^{20}=-73.9^{\circ}\left(c=1.0, \mathrm{CHCl}_{3}\right)$.

Physical properties: white solid; Melting Point: $66.2-67.5^{\circ} \mathrm{C}$; Yield: $42 \%, 15.8 \mathrm{mg}$; Reaction time: $73.5 \mathrm{~h}$.

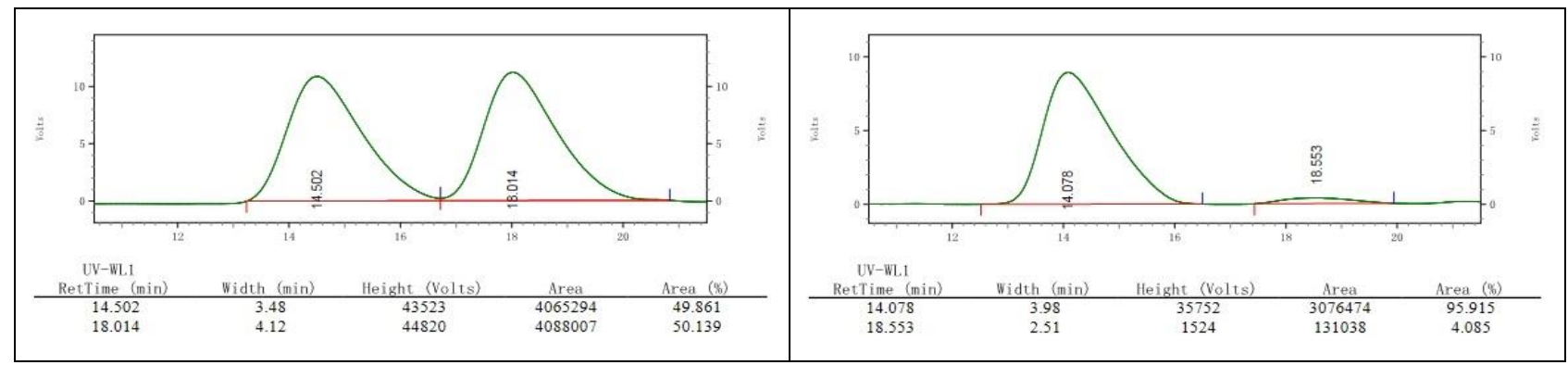

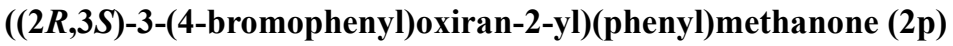

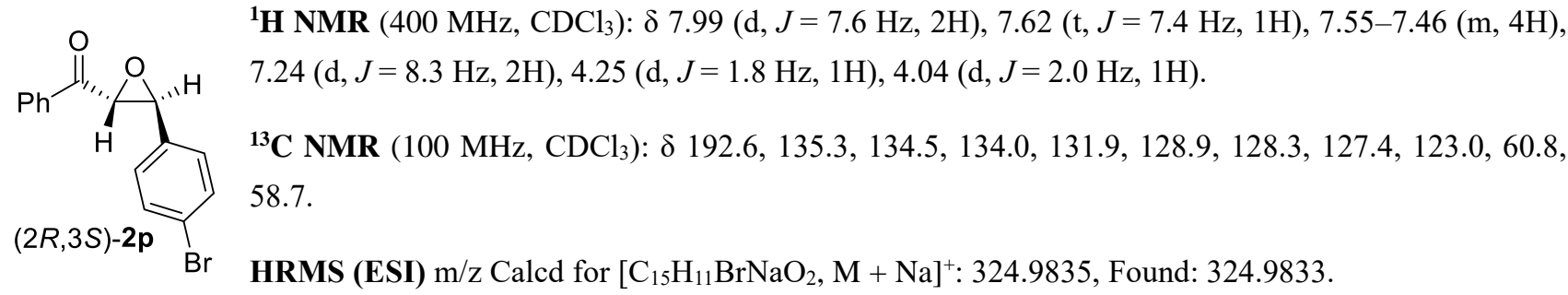

HPLC analysis: Chiralcel IA-H (Hexane $/ i-\mathrm{PrOH}=90: 10$, flow rate $=1.0 \mathrm{~mL} / \mathrm{min}$, wave length $=210 \mathrm{~nm}), t_{\mathrm{R}}=10.510$ $\min \left(\right.$ minor), $t_{\mathrm{R}}=11.913 \min$ (major).

Optical Rotation: $[\alpha]_{\mathrm{D}}^{20}=-93.0^{\circ}\left(c=1.0, \mathrm{CHCl}_{3}\right)$.

Physical properties: white solid; Melting Point: $89.3-90.8^{\circ} \mathrm{C}$; Yield: $51 \%, 15.5 \mathrm{mg}$; Reaction time: $73.5 \mathrm{~h}$.

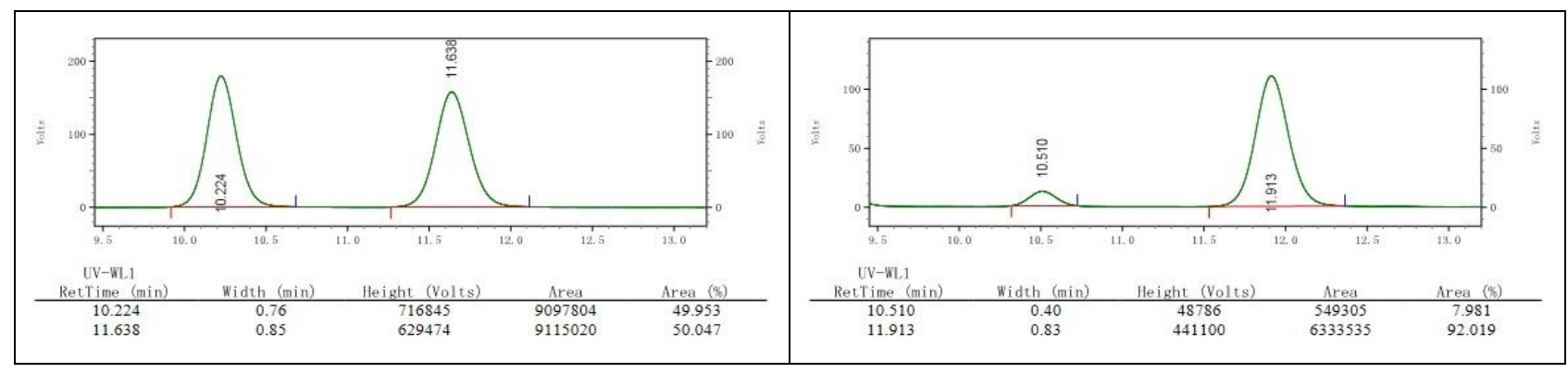


<smiles>CC(C)(C)OOC(CC(=O)c1ccccc1)c1ccccc1Cl</smiles>

$(S)-19$

${ }^{1} \mathbf{H}$ NMR $\left(400 \mathrm{MHz}, \mathrm{CDCl}_{3}\right): \delta 7.99(\mathrm{~d}, J=7.4 \mathrm{~Hz}, 2 \mathrm{H}), 7.61(\mathrm{~d}, J=7.7 \mathrm{~Hz}, 1 \mathrm{H}), 7.56(\mathrm{t}, J=$ $7.4 \mathrm{~Hz}, 1 \mathrm{H}), 7.46(\mathrm{t}, J=7.6 \mathrm{~Hz}, 2 \mathrm{H}), 7.35(\mathrm{~d}, J=7.9 \mathrm{~Hz}, 1 \mathrm{H}), 7.30(\mathrm{t}, J=7.4 \mathrm{~Hz}, 1 \mathrm{H}), 7.23(\mathrm{td}$, $J=7.7,1.8 \mathrm{~Hz}, 1 \mathrm{H}), 6.03(\mathrm{dd}, J=8.9,3.8 \mathrm{~Hz}, 1 \mathrm{H}), 3.47(\mathrm{dd}, J=16.2,8.9 \mathrm{~Hz}, 1 \mathrm{H}), 3.24(\mathrm{dd}, J=$ $16.2,3.9 \mathrm{~Hz}, 1 \mathrm{H}), 1.16(\mathrm{~s}, 9 \mathrm{H})$.

${ }^{13}$ C NMR $\left(100 \mathrm{MHz}, \mathrm{CDCl}_{3}\right): \delta 196.5,138.2,137.0,133.1,132.3,129.5,128.8,128.5,128.3$, $127.9,126.9,81.0,78.7,43.1,26.3$.

HRMS (ESI) $\mathrm{m} / \mathrm{z}$ Calcd for $\left[\mathrm{C}_{19} \mathrm{H}_{21} \mathrm{ClNaO}_{3}, \mathrm{M}+\mathrm{Na}^{+}:\right.$355.1071, Found: 355.1077.

HPLC analysis: Chiralcel OJ-H (Hexane $/ i-\mathrm{PrOH}=99: 1$, flow rate $=1.0 \mathrm{~mL} / \mathrm{min}$, wave length $=254 \mathrm{~nm}), t_{\mathrm{R}}=7.404 \mathrm{~min}$ (minor), $t_{\mathrm{R}}=11.254 \mathrm{~min}$ (major).

Optical Rotation: $[\alpha]_{\mathrm{D}}^{20}=-45.5^{\circ}\left(c=1.0, \mathrm{CHCl}_{3}\right)$.

Physical properties: pale yellow solid; Melting Point: $66.1-67.9^{\circ} \mathrm{C}$; Yield: $40 \%, 13.3 \mathrm{mg}$; Reaction time: $48.0 \mathrm{~h}$.

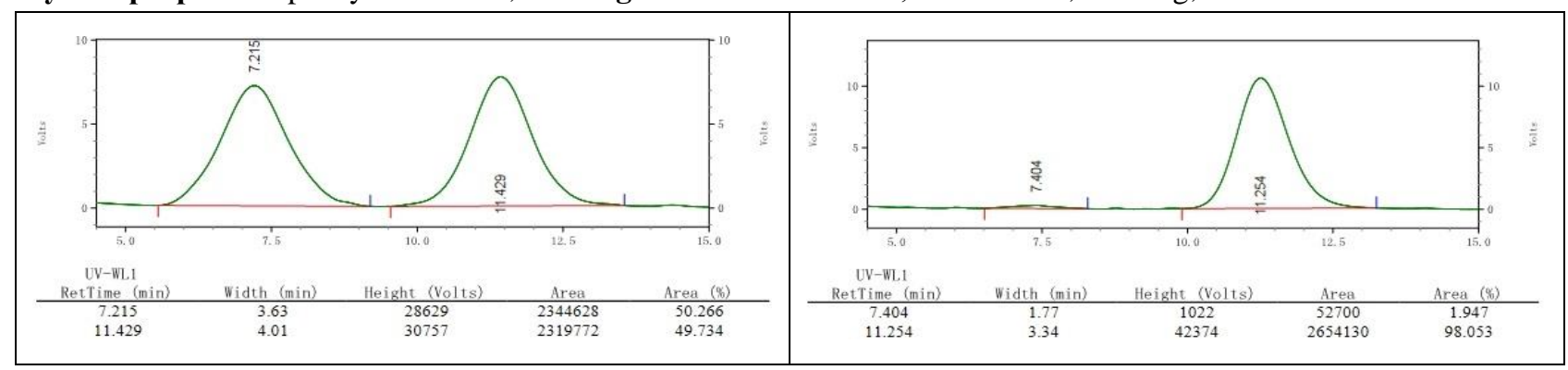

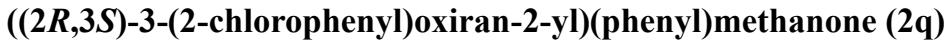<smiles>O=C(c1ccccc1)C1O[C@H]1c1ccccc1Cl</smiles>

$(2 R, 3 S)-2 q$

${ }^{1}$ H NMR $\left(400 \mathrm{MHz}, \mathrm{CDCl}_{3}\right): \delta 8.05(\mathrm{~d}, J=7.9 \mathrm{~Hz}, 2 \mathrm{H}), 7.62(\mathrm{t}, J=7.4 \mathrm{~Hz}, 1 \mathrm{H}), 7.50(\mathrm{t}, J=7.7 \mathrm{~Hz}$, 2H), 7.39 (dq, $J=7.6,3.9 \mathrm{~Hz}, 2 \mathrm{H}), 7.34-7.28(\mathrm{~m}, 2 \mathrm{H}), 4.40$ (d, $J=1.8 \mathrm{~Hz}, 1 \mathrm{H}), 4.17$ (d, $J=1.9 \mathrm{~Hz}$, $1 \mathrm{H})$.

${ }^{13}$ C NMR (100 MHz, $\left.\mathrm{CDCl}_{3}\right): \delta 192.6,135.2,134.0,133.6,133.2,129.7,129.2,128.8,128.3,127.2$, 126.0, 59.9, 57.0.

HRMS (ESI) $\mathrm{m} / \mathrm{z}$ Calcd for $\left[\mathrm{C}_{15} \mathrm{H}_{11} \mathrm{ClNaO}_{2}, \mathrm{M}+\mathrm{Na}^{+}:\right.$281.0340, Found: 281.0346.

HPLC analysis: Chiralcel AD-H (Hexane $/ i-\mathrm{PrOH}=97: 3$, flow rate $=1.0 \mathrm{~mL} / \mathrm{min}$, wave length $=254 \mathrm{~nm}), t_{\mathrm{R}}=12.333$ $\min$ (major), $t_{\mathrm{R}}=13.412 \min$ (minor).

Optical Rotation: $[\alpha]_{\mathrm{D}}^{20}=-62.3^{\circ}\left(c=1.0, \mathrm{CHCl}_{3}\right)$.

Physical properties: white solid; Melting Point: $73.5-74.2^{\circ} \mathrm{C}$; Yield: $49 \%, 12.7 \mathrm{mg}$; Reaction time: $48.0 \mathrm{~h}$.

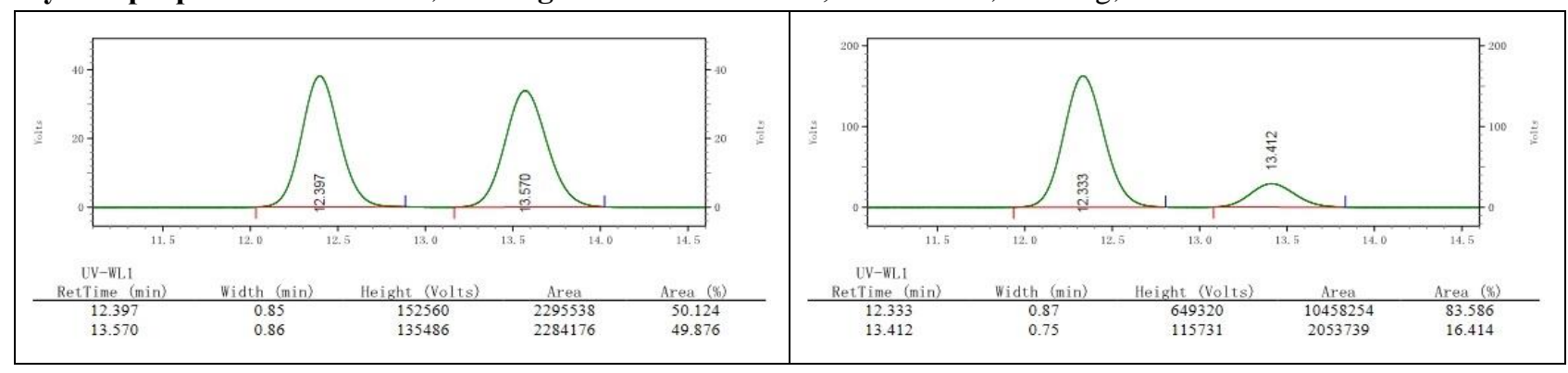


(S)-3-(3-bromophenyl)-3-(tert-butylperoxy)-1-phenylpropan-1-one (1r)<smiles>CC(C)(C)OOC(CC(=O)c1ccccc1)c1cccc(Br)c1</smiles>

$(S)-1 \mathbf{r}$

${ }^{1} \mathbf{H}$ NMR (400 MHz, CDCl $): \delta 7.95$ (d, $\left.J=7.3 \mathrm{~Hz}, 2 \mathrm{H}\right), 7.61-7.53(\mathrm{~m}, 2 \mathrm{H}), 7.49-7.39$ (m, 3H), $7.36(\mathrm{~d}, J=7.7 \mathrm{~Hz}, 1 \mathrm{H}), 7.21(\mathrm{t}, J=7.8 \mathrm{~Hz}, 1 \mathrm{H}), 5.59-5.52(\mathrm{~m}, 1 \mathrm{H}), 3.71(\mathrm{dd}, J=16.5,7.3 \mathrm{~Hz}$, $1 \mathrm{H}), 3.15(\mathrm{dd}, J=16.5,5.7 \mathrm{~Hz}, 1 \mathrm{H}), 1.16(\mathrm{~s}, 9 \mathrm{H})$.

${ }^{13}$ C NMR (100 MHz, $\left.\mathrm{CDCl}_{3}\right): \delta 196.8,142.7,136.8,133.2,131.0,130.0,129.9,128.5,128.2$, $125.7,122.4,81.4,80.9,43.8,26.3$.

HRMS (ESI) m/z Calcd for $\left[\mathrm{C}_{19} \mathrm{H}_{21} \mathrm{BrNaO}_{3}, \mathrm{M}+\mathrm{Na}\right]^{+}:$399.0566, Found: 399.0571.

HPLC analysis: Chiralcel OJ-H (Hexane $/ i-\mathrm{PrOH}=90: 10$, flow rate $=1.0 \mathrm{~mL} / \mathrm{min}$, wave length $=210 \mathrm{~nm}), t_{\mathrm{R}}=5.183 \mathrm{~min}$ (minor), $t_{\mathrm{R}}=6.147 \mathrm{~min}$ (major).

Optical Rotation: $[\alpha]_{\mathrm{D}}^{20}=-46.2^{\circ}\left(c=1.0, \mathrm{CHCl}_{3}\right)$.

Physical properties: white solid; Melting Point: $51.1-51.8^{\circ} \mathrm{C}$; Yield: $42 \%, 15.8 \mathrm{mg}$; Reaction time: $68.5 \mathrm{~h}$.

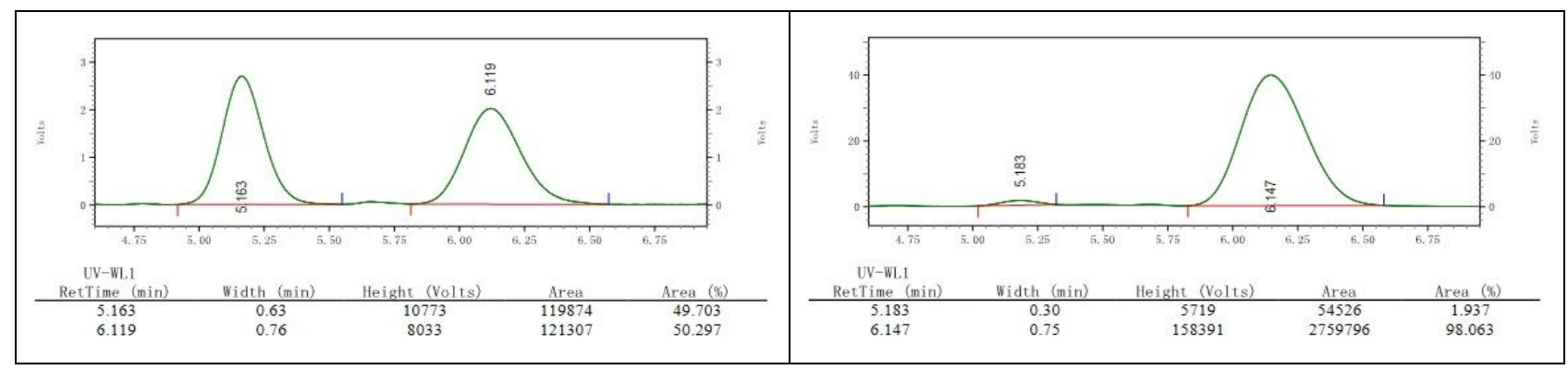

((2R,3S)-3-(3-bromophenyl)oxiran-2-yl)(phenyl)methanone (2r)<smiles>O=C(c1ccccc1)[C@H]1O[C@H]1c1cccc(Br)c1</smiles>

$(2 R, 3 S)-2 r$

${ }^{1}$ H NMR (400 MHz, CDCl $)$ ): $\delta 7.98(\mathrm{~d}, J=7.4 \mathrm{~Hz}, 2 \mathrm{H}), 7.61(\mathrm{t}, J=7.4 \mathrm{~Hz}, 1 \mathrm{H}), 7.51-7.45(\mathrm{~m}$, $4 \mathrm{H}), 7.30(\mathrm{~d}, J=7.7 \mathrm{~Hz}, 1 \mathrm{H}), 7.24(\mathrm{~d}, J=7.7 \mathrm{~Hz}, 1 \mathrm{H}), 4.27(\mathrm{~d}, J=1.7 \mathrm{~Hz}, 1 \mathrm{H}), 4.03(\mathrm{~d}, J=1.8$ $\mathrm{Hz}, 1 \mathrm{H})$.

${ }^{13} \mathrm{C}$ NMR (100 MHz, $\left.\mathrm{CDCl}_{3}\right): \delta$ 192.5, 137.8, 135.2, 134.0, 132.0, 130.2, 128.8, 128.5, 128.3, 124.5, $122.8,60.7,58.3$.

HRMS (ESI) $\mathrm{m} / \mathrm{z}$ Calcd for $\left[\mathrm{C}_{15} \mathrm{H}_{11} \mathrm{BrNaO}_{2}, \mathrm{M}+\mathrm{Na}\right]^{+}: 324.9835$, Found: 324.9841.

HPLC analysis: Chiralcel IA-H (Hexane $/ i-\mathrm{PrOH}=95: 5$, flow rate $=1.0 \mathrm{~mL} / \mathrm{min}$, wave length $=254 \mathrm{~nm}), t_{\mathrm{R}}=11.174 \mathrm{~min}$ (major), $t_{\mathrm{R}}=15.020 \mathrm{~min}$ (minor).

Optical Rotation: $[\alpha]_{\mathrm{D}}^{20}=-85.2^{\circ}\left(c=1.0, \mathrm{CHCl}_{3}\right)$.

Physical properties: white solid; Melting Point: $69.8-71.2^{\circ} \mathrm{C}$; Yield: $48 \%, 14.5 \mathrm{mg}$; Reaction time: $68.5 \mathrm{~h}$.

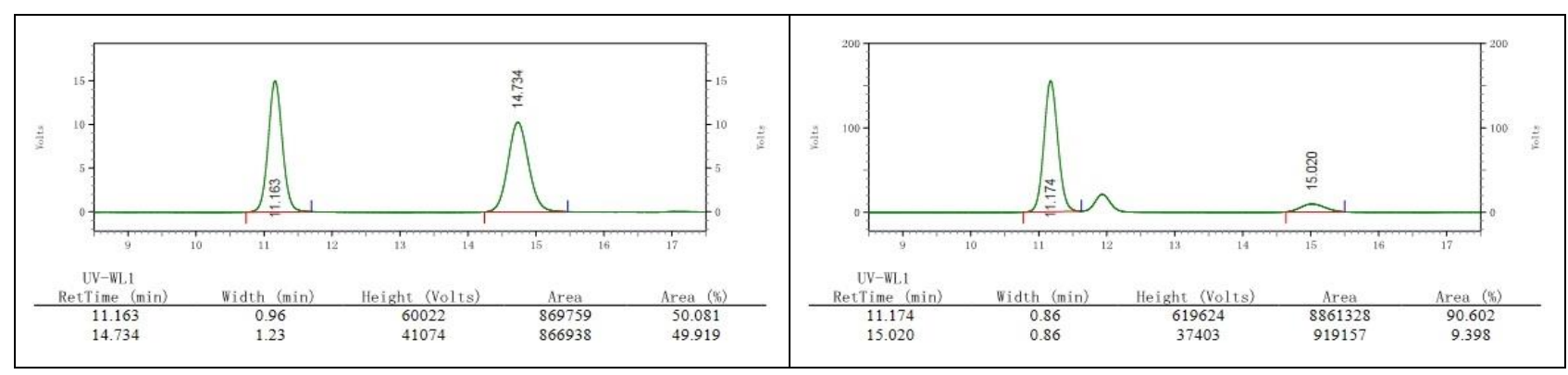


(S)-1,3-diphenyl-3-((2-phenylpropan-2-yl)peroxy)propan-1-one (1s)<smiles>CC(C)(OOC(CC(=O)c1ccccc1)c1ccccc1)c1ccccc1</smiles>

$(S)-1 s$

${ }^{1} \mathbf{H}$ NMR $\left(400 \mathrm{MHz}, \mathrm{CDCl}_{3}\right): \delta 7.83(\mathrm{~d}, J=7.4 \mathrm{~Hz}, 2 \mathrm{H}), 7.45(\mathrm{t}, J=7.4 \mathrm{~Hz}, 1 \mathrm{H}), 7.37-7.17$ $(\mathrm{m}, 12 \mathrm{H}), 5.59(\mathrm{t}, J=6.5 \mathrm{~Hz}, 1 \mathrm{H}), 3.62(\mathrm{dd}, J=16.3,7.2 \mathrm{~Hz}, 1 \mathrm{H}), 3.11(\mathrm{dd}, J=16.3,5.8 \mathrm{~Hz}$, $1 \mathrm{H}), 1.51(\mathrm{~s}, 3 \mathrm{H}), 1.46(\mathrm{~s}, 3 \mathrm{H})$.

${ }^{13}$ C NMR (100 MHz, $\left.\mathrm{CDCl}_{3}\right): \delta 197.0,145.1,139.9,136.9,132.9,128.3,128.2,128.1,127.9$, $127.8,127.0,126.8,125.4,83.1,82.0,43.7,26.7,26.2$.

HRMS (ESI) $\mathrm{m} / \mathrm{z}$ Calcd for $\left[\mathrm{C}_{24} \mathrm{H}_{24} \mathrm{NaO}_{3}, \mathrm{M}+\mathrm{Na}\right]^{+}: 383.1618$, Found: 383.1623 .

HPLC analysis: Chiralcel IA-H (Hexane $/ i-\mathrm{PrOH}=98: 2$, flow rate $=1.0 \mathrm{~mL} / \mathrm{min}$, wave length $=254 \mathrm{~nm}), t_{\mathrm{R}}=7.472 \mathrm{~min}$ (major), $t_{\mathrm{R}}=8.145 \mathrm{~min}$ (minor).

Optical Rotation: $[\alpha]_{\mathrm{D}}^{20}=-30.1^{\circ}\left(c=1.0, \mathrm{CHCl}_{3}\right)$.

Physical properties: colorless oil; Yield: $41 \%, 14.8 \mathrm{mg}$; Reaction time: $15.0 \mathrm{~h}$.

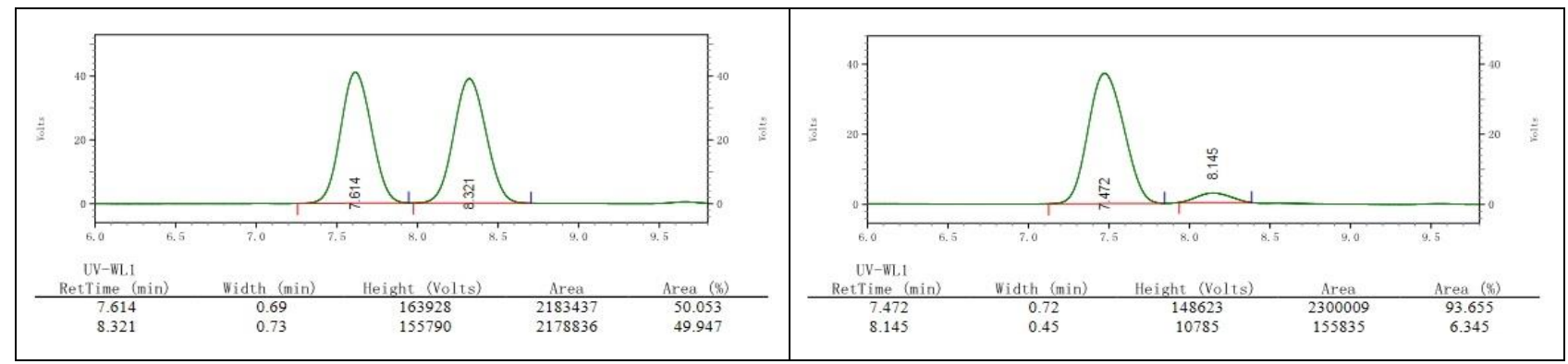

phenyl((2R,3S)-3-phenyloxiran-2-yl)methanone (2s)<smiles>O=C(c1ccccc1)C1OC1c1ccccc1</smiles>

$(2 R, 3 S)-2 \mathrm{~s}$

${ }^{1} \mathbf{H}$ NMR $\left(400 \mathrm{MHz}, \mathrm{CDCl}_{3}\right): \delta 8.01(\mathrm{~d}, J=7.5 \mathrm{~Hz}, 2 \mathrm{H}), 7.61(\mathrm{t}, J=7.3 \mathrm{~Hz}, 1 \mathrm{H}), 7.48(\mathrm{t}, J=7.6 \mathrm{~Hz}$, $2 \mathrm{H}), 7.42-7.35(\mathrm{~m}, 5 \mathrm{H}), 4.30(\mathrm{~d}, J=1.9 \mathrm{~Hz}, 1 \mathrm{H}), 4.07(\mathrm{~d}, J=2.0 \mathrm{~Hz}, 1 \mathrm{H})$.

${ }^{13}$ C NMR (100 MHz, $\left.\mathrm{CDCl}_{3}\right): \delta$ 193.0, 135.4, 135.4, 134.0, 129.0, 128.8, 128.7, 128.3, 60.9, 59.3.

HRMS (ESI) m/z Calcd for $\left[\mathrm{C}_{15} \mathrm{H}_{12} \mathrm{NaO}_{2}, \mathrm{M}+\mathrm{Na}\right]^{+}:$247.0730, Found: 247.0734.

HPLC analysis: Chiralcel $\mathrm{AD}-\mathrm{H}(\mathrm{Hexane} / i-\mathrm{PrOH}=85: 15$, flow rate $=1.0 \mathrm{~mL} / \mathrm{min}$, wave length $=254$ $\mathrm{nm}), t_{\mathrm{R}}=8.873 \min \left(\right.$ minor), $t_{\mathrm{R}}=9.362 \mathrm{~min}$ (major).

Optical Rotation: $[\alpha]_{\mathrm{D}}^{20}=-81.9^{\circ}\left(c=1.0, \mathrm{CHCl}_{3}\right)$.

Physical properties: white solid; Melting Point: $89.7-90.8^{\circ} \mathrm{C}$; Yield: $46 \%, 10.3 \mathrm{mg}$; Reaction time: $15.0 \mathrm{~h}$.

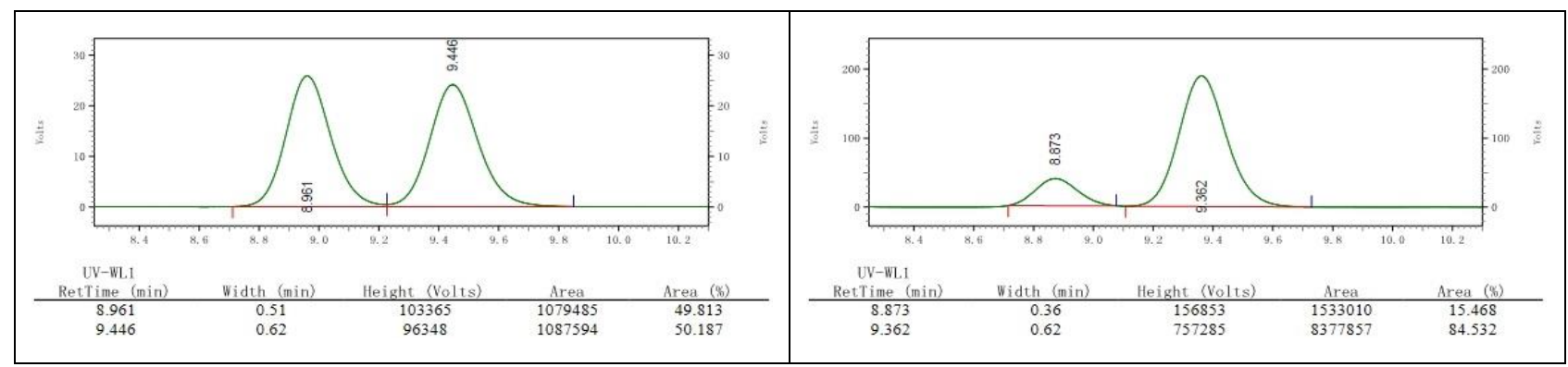




\section{VII. ${ }^{1} \mathrm{H},{ }^{13} \mathrm{C}$ NMR, HRMS data and HPLC traces of compounds $(3,4,5,6,7)$}

(3S)-3-(4-bromophenyl)-3-(tert-butylperoxy)-1-phenylpropan-1-ol (3)<smiles>CC(C)(C)OO[C@H](CC(O)c1ccccc1)c1ccc(Br)cc1</smiles>

${ }^{1} \mathbf{H}$ NMR (400 MHz, $\left.\mathrm{CDCl}_{3}\right): \delta 7.45(\mathrm{~d}, J=8.3 \mathrm{~Hz}, 2 \mathrm{H}), 7.37-7.27(\mathrm{~m}, 4 \mathrm{H}), 7.27-7.22(\mathrm{~m}, 1 \mathrm{H})$, $7.20(\mathrm{~d}, J=8.3 \mathrm{~Hz}, 2 \mathrm{H}), 5.01(\mathrm{dd}, J=8.2,5.9 \mathrm{~Hz}, 1 \mathrm{H}), 4.69(\mathrm{dd}, J=9.4,3.8 \mathrm{~Hz}, 1 \mathrm{H}), 2.78(\mathrm{~s}$, 1H), 2.40-2.29 (m, 1H), $1.91(\mathrm{~m}, 1 \mathrm{H}), 1.18(\mathrm{~s}, 9 \mathrm{H})$.

${ }^{13} \mathbf{C ~ N M R}\left(100 \mathrm{MHz}, \mathrm{CDCl}_{3}\right): \delta 143.8,139.9,131.3,128.7,128.4,127.6,125.7,121.6,83.8$, $80.4,72.2,44.4,26.4$.

HRMS (ESI) m/z Calcd for $\left[\mathrm{C}_{19} \mathrm{H}_{23} \mathrm{BrNaO}_{3}, \mathrm{M}+\mathrm{Na}\right]^{+}:$401.0723, Found: 401.0731.

HPLC analysis: Chiralcel OD-H $($ Hexane $/ i-\mathrm{PrOH}=95: 5$, flow rate $=1.0 \mathrm{~mL} / \mathrm{min}$, wave length $=220 \mathrm{~nm}), t_{\mathrm{R}}=7.521 \mathrm{~min}$ (major), $t_{\mathrm{R}}=8.599 \min$ (minor).

Optical Rotation: $[\alpha]_{\mathrm{D}}^{20}=-57.2^{\circ}\left(c=1.0, \mathrm{CHCl}_{3}\right)$.

Physical properties: colorless oil; Yield: $80 \%, 30.3 \mathrm{mg}$.

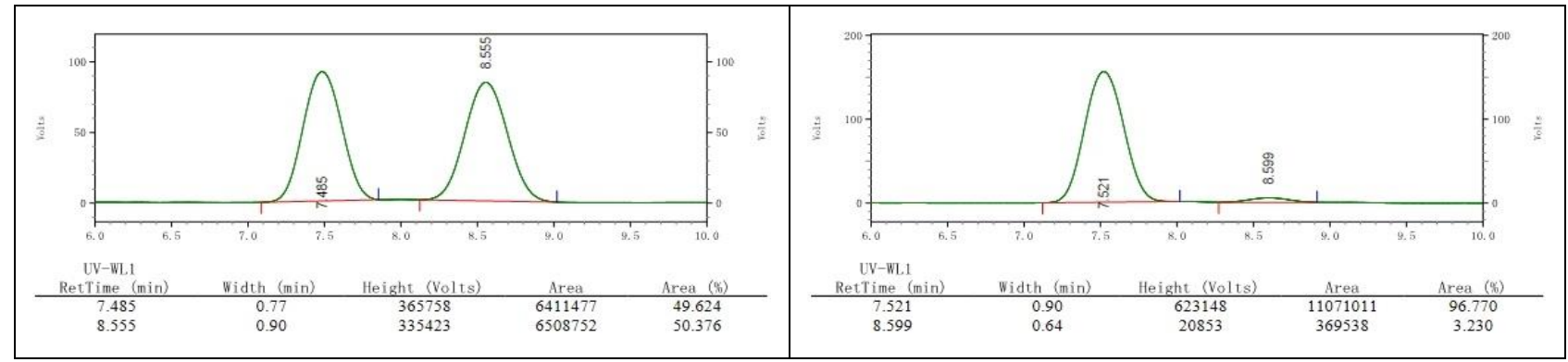

(S)-1-(3-(4-bromophenyl)-3-(tert-butylperoxy)-1-phenylpropylidene)-2-(2,4-dinitrophenyl)hydrazine (4)<smiles>CC(C)(C)OO[C@H](C/C(=N/Nc1ccc([N+](=O)[O-])cc1[N+](=O)[O-])c1ccccc1)c1ccc(Br)cc1</smiles>

4
${ }^{1} \mathbf{H}$ NMR (400 MHz, $\left.\mathrm{CDCl}_{3}\right): \delta 11.57(\mathrm{~s}, 5 \mathrm{H}), 11.20(\mathrm{~s}, 1 \mathrm{H}), 9.14(\mathrm{~d}, J=2.0 \mathrm{~Hz}, 5 \mathrm{H})$, $9.00(\mathrm{~d}, J=2.0 \mathrm{~Hz}, 1 \mathrm{H}), 8.33(\mathrm{dd}, J=9.5,2.6 \mathrm{~Hz}, 5 \mathrm{H}), 8.29-8.26(\mathrm{~m}, 1 \mathrm{H}), 8.08(\mathrm{~d}, J=$ 9.5 Hz, 5H), 7.93-7.87 (m, 10H), 7.59-7.45 (m, 30H), 7.39-7.35 (m, 9H), 7.33-7.23 (m, 6H), 5.23-5.19 (m, 1H), 5.17 (dd, $J=9.7,4.2 \mathrm{~Hz}, 5 \mathrm{H}), 3.43-3.38$ (m, 1H), 3.34 $(\mathrm{dd}, J=14.7,10.0 \mathrm{~Hz}, 5 \mathrm{H}), 3.16(\mathrm{dd}, J=14.9,4.0 \mathrm{~Hz}, 5 \mathrm{H}), 3.12-3.07$ (m, 1H), 1.16 (s, 9H), $1.04(\mathrm{~s}, 45 \mathrm{H})$.

${ }^{13} \mathrm{C}$ NMR $\left(100 \mathrm{MHz} \mathrm{CDCl}_{3}\right): \delta 155.0,152.5,145.0,144.6,138.9,138.8,138.2,137.8$, 136.2, 133.0, 131.8, 131.4, 130.4, 130.3, 130.0, 129.9, 129.8, 129.6, 129.0, 128.8, 127.9, 126.7, 123.5, 123.3, 122.3, $122.1,116.6,116.3,82.8,82.1,81.2,80.5,42.9,34.2,26.4,26.1$.

Physical properties: red solid; Yield: $87 \%, 48.4 \mathrm{mg}$. 
(S,E)-1-(3-(4-bromophenyl)-3-(tert-butylperoxy)-1-phenylpropylidene)-2-(2,4-dinitrophenyl)hydrazine ((E)-4a)<smiles>CCO/C(CC(CCC(Br)c1ccccc1)OC(C)(C)C)=N\Nc1ccc([N+](=O)[O-])cc1[N+](=O)[O-]</smiles>

${ }^{1} \mathbf{H}$ NMR $\left(400 \mathrm{MHz}, \mathrm{CDCl}_{3}\right): \delta 11.58(\mathrm{~s}, 1 \mathrm{H}), 9.15(\mathrm{~d}, J=2.5 \mathrm{~Hz}, 1 \mathrm{H}), 8.34(\mathrm{dd}, J=9.5,2.6 \mathrm{~Hz}$, $1 \mathrm{H}), 8.08(\mathrm{~d}, J=9.5 \mathrm{~Hz}, 1 \mathrm{H}), 7.90(\mathrm{dd}, J=6.7,3.1 \mathrm{~Hz}, 2 \mathrm{H}), 7.62-7.44(\mathrm{~m}, 5 \mathrm{H}), 7.37(\mathrm{~d}, J=8.0$ $\mathrm{Hz}, 2 \mathrm{H}), 5.17(\mathrm{dd}, J=9.5,3.8 \mathrm{~Hz}, 1 \mathrm{H}), 3.34(\mathrm{dd}, J=14.7,10.2 \mathrm{~Hz}, 1 \mathrm{H}), 3.16(\mathrm{dd}, J=14.9,3.8$ $\mathrm{Hz}, 1 \mathrm{H}), 1.04(\mathrm{~s}, 9 \mathrm{H})$.

${ }^{13} \mathbf{C}$ NMR $\left(100 \mathrm{MHz}, \mathrm{CDCl}_{3}\right): \delta 152.5,145.0,138.9,138.2,136.1,131.8,130.3,130.0,129.6$, $128.8,127.9,126.7,123.5,122.3,116.6,82.8,81.2,34.2,26.1$.

HRMS (ESI) m/z Calcd for $\left[\mathrm{C}_{25} \mathrm{H}_{24} \mathrm{BrN}_{4} \mathrm{O}_{6}, \mathrm{M}-\mathrm{H}\right]$ ]: 555.0885, Found: 555.0898.

HPLC analysis: Chiralcel IA-H $($ Hexane $/ i-\mathrm{PrOH}=90: 10$, flow rate $=1.0 \mathrm{~mL} / \mathrm{min}$, wave length $=254 \mathrm{~nm}), t_{\mathrm{R}}=6.861 \mathrm{~min}$ (major), $t_{\mathrm{R}}=7.213 \mathrm{~min}$ (minor).

Optical Rotation: $[\alpha]_{\mathrm{D}}^{20}=-122.8^{\circ}\left(c=0.5, \mathrm{CHCl}_{3}\right)$.

Physical properties: red solid; Melting Point: $168.7-169.5^{\circ} \mathrm{C}$; Yield: $73 \%$, $40.6 \mathrm{mg}$.

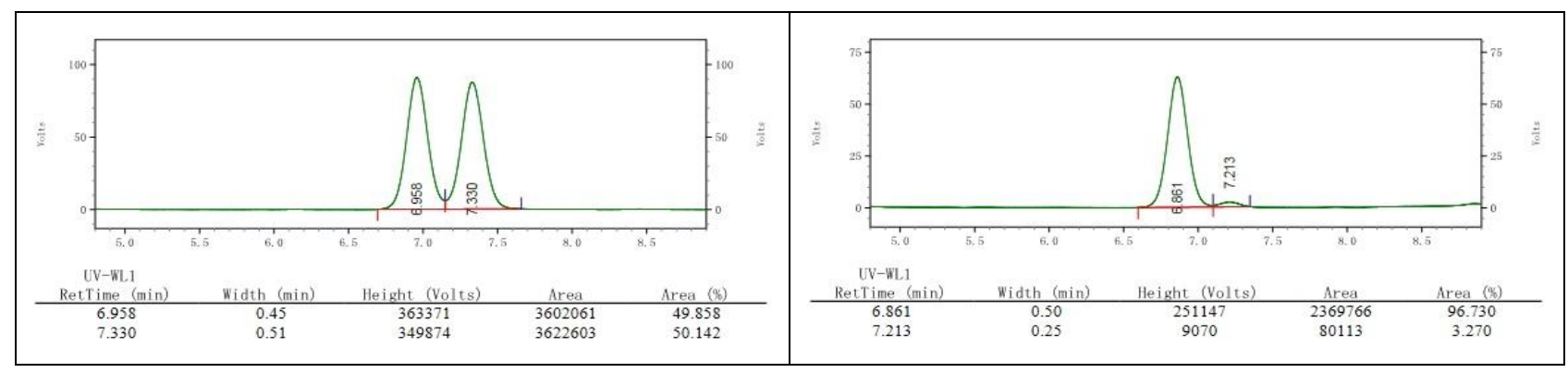

(S,Z)-1-(3-(4-bromophenyl)-3-(tert-butylperoxy)-1-phenylpropylidene)-2-(2,4-dinitrophenyl)hydrazine ((Z)-4b)<smiles>CC(C)(C)OO[C@H](C/C(=N/Nc1ccc([N+](=O)[O-])cc1[N+](=O)[O-])c1ccccc1)c1ccc(Br)cc1</smiles>

${ }^{1} \mathbf{H}$ NMR (400 MHz, $\left.\mathrm{CDCl}_{3}\right): \delta 11.20(\mathrm{~s}, 1 \mathrm{H}), 9.02(\mathrm{dd}, J=8.0,2.7 \mathrm{~Hz}, 1 \mathrm{H}), 8.32-8.25$ $(\mathrm{m}, 1 \mathrm{H}), 7.92(\mathrm{~d}, J=9.6 \mathrm{~Hz}, 1 \mathrm{H}), 7.57(\mathrm{qd}, J=7.8,6.8,3.7 \mathrm{~Hz}, 3 \mathrm{H}), 7.47(\mathrm{~d}, J=8.3 \mathrm{~Hz}$, 2H), 7.34-7.28 (m, 2H), 7.25 (d, $J=8.7 \mathrm{~Hz}, 2 \mathrm{H}), 5.18(\mathrm{td}, J=7.1,2.3 \mathrm{~Hz}, 1 \mathrm{H}), 3.39$ (dd, $J=15.3,7.4 \mathrm{~Hz}, 1 \mathrm{H}), 3.10(\mathrm{dd}, J=14.7,6.0 \mathrm{~Hz}, 1 \mathrm{H}), 1.15(\mathrm{~s}, 9 \mathrm{H})$.

${ }^{13} \mathrm{C}$ NMR $\left(100 \mathrm{MHz}, \mathrm{CDCl}_{3}\right): \delta 155.1,144.7,138.8,138.8,137.8,133.0,131.5,130.5$, $129.9,129.1,129.0,126.7,123.3,122.1,116.3,82.1,80.5,43.0,26.4$.

HRMS (ESI) m/z Calcd for $\left[\mathrm{C}_{25} \mathrm{H}_{24} \mathrm{BrN}_{4} \mathrm{O}_{6}, \mathrm{M}\right.$ - H] : 555.0885 , Found: 555.0894.

HPLC analysis: Chiralcel IA-H $(\mathrm{Hexane} / i-\mathrm{PrOH}=90: 10$, flow rate $=1.0 \mathrm{~mL} / \mathrm{min}$, wave length $=254 \mathrm{~nm}), t_{\mathrm{R}}=6.895 \mathrm{~min}$ (minor), $t_{\mathrm{R}}=7.253 \mathrm{~min}$ (major).

Physical properties: yellow solid; Melting Point: $173.0-174.9^{\circ} \mathrm{C}$; Yield: $14 \%, 7.8 \mathrm{mg}$. 


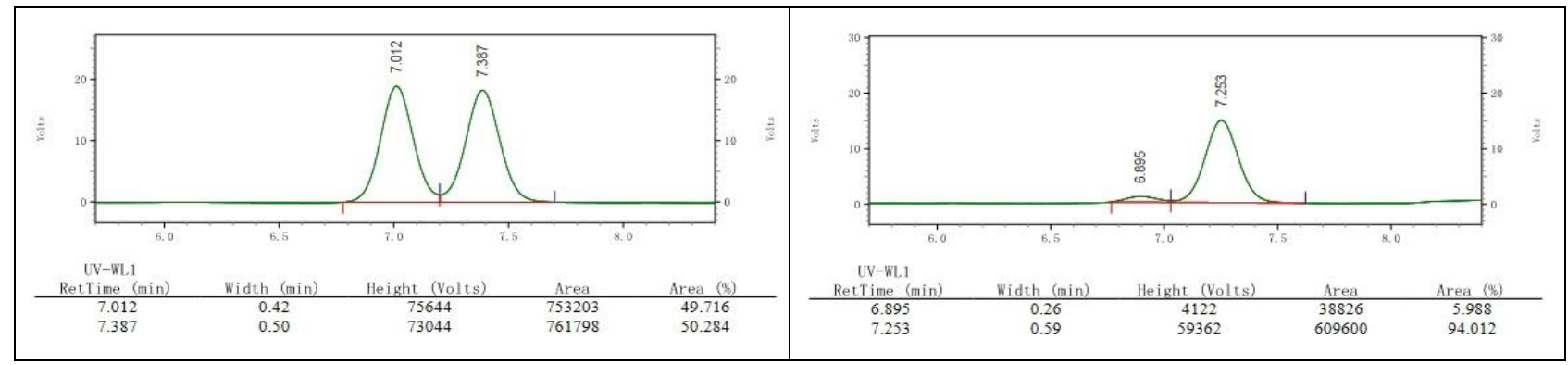

(R)-((2S,3S)-3-(4-bromophenyl)oxiran-2-yl)(phenyl)methanol (5)<smiles>OC(CO[C@@H](c1ccccc1)c1ccc(Br)cc1)c1ccccc1</smiles>

${ }^{1} \mathbf{H}$ NMR (400 MHz, $\left.\mathrm{CDCl}_{3}\right): \delta 7.49-7.27(\mathrm{~m}, 7 \mathrm{H}), 7.09(\mathrm{~d}, J=8.1 \mathrm{~Hz}, 2 \mathrm{H}), 4.94(\mathrm{~s}, 1 \mathrm{H}), 4.07(\mathrm{~d}, J=$ $1.2 \mathrm{~Hz}, 1 \mathrm{H}), 3.21(\mathrm{t}, J=2.6 \mathrm{~Hz}, 1 \mathrm{H}), 2.72(\mathrm{~d}, J=1.6 \mathrm{~Hz}, 1 \mathrm{H})$.

${ }^{13}$ C NMR (100 MHz, $\left.\mathrm{CDCl}_{3}\right): \delta 139.0,135.6,131.5,128.7,128.4,127.3,126.4,122.1,71.1,65.0,54.4$. HRMS (ESI) m/z Calcd for $\left[\mathrm{C}_{15} \mathrm{H}_{12} \mathrm{BrO}_{2}, \mathrm{M}-\mathrm{H}\right]^{-:}: 303.0026$, Found: 303.0029.

HPLC analysis: Chiralcel IA-H (Hexane $/ i-\mathrm{PrOH}=90: 10$, flow rate $=1.0 \mathrm{~mL} / \mathrm{min}$, wave length $=210$ $\mathrm{nm}), t_{\mathrm{R}}=12.051 \mathrm{~min}$ (minor), $t_{\mathrm{R}}=14.090 \mathrm{~min}$ (major).

Optical Rotation: $[\alpha]_{\mathrm{D}}^{20}=-28.3^{\circ}\left(c=0.2, \mathrm{CHCl}_{3}\right)$.

Physical properties: white solid; Melting Point: $79.6-80.5^{\circ} \mathrm{C}$; Yield: $93 \%, 28.4 \mathrm{mg}$.

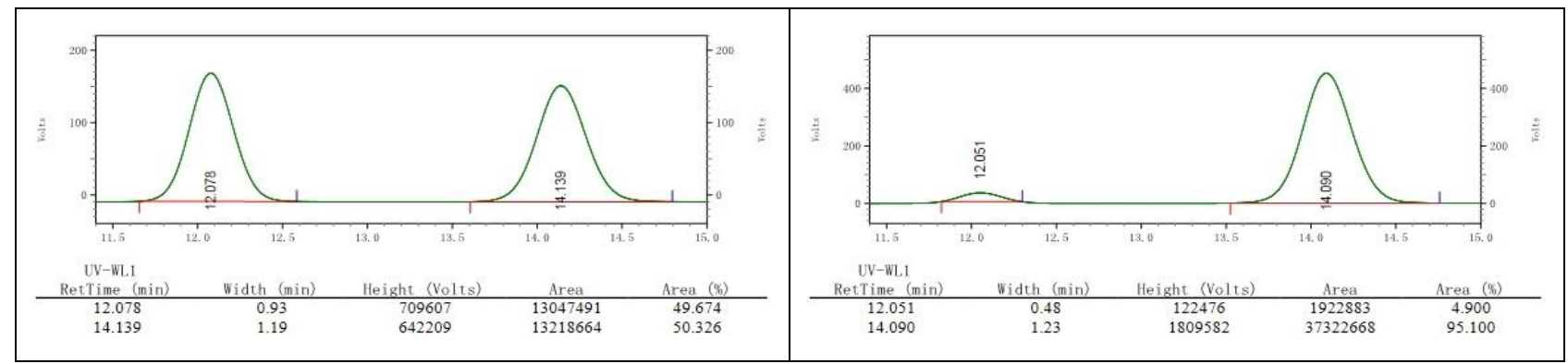

(2S,3S)-3-(4-bromophenyl)-3-chloro-2-hydroxy-1-phenylpropan-1-one (6)<smiles>O=C(c1ccccc1)[C@H](O)[C@H](Cl)c1ccc(Br)cc1</smiles>

6

${ }^{1} \mathbf{H}$ NMR (400 MHz, $\left.\mathrm{CDCl}_{3}\right): \delta 7.91(\mathrm{~d}, J=7.5 \mathrm{~Hz}, 2 \mathrm{H}), 7.68(\mathrm{t}, J=7.3 \mathrm{~Hz}, 1 \mathrm{H}), 7.55(\mathrm{t}, J=$ $7.6 \mathrm{~Hz}, 2 \mathrm{H}), 7.50(\mathrm{~d}, J=8.3 \mathrm{~Hz}, 2 \mathrm{H}), 7.42(\mathrm{~d}, J=8.3 \mathrm{~Hz}, 2 \mathrm{H}), 5.35(\mathrm{~d}, J=5.7 \mathrm{~Hz}, 1 \mathrm{H}), 5.19$ $(\mathrm{s}, 1 \mathrm{H}), 4.16(\mathrm{~d}, J=7.0 \mathrm{~Hz}, 1 \mathrm{H})$.

${ }^{13} \mathrm{C}$ NMR (100 MHz, $\left.\mathrm{CDCl}_{3}\right): \delta 197.3,137.1,134.4,133.4,131.6,129.6,129.2,128.5,122.9$, 75.7, 62.9 .

HRMS (ESI) m/z Calcd for $\left[\mathrm{C}_{15} \mathrm{H}_{12} \mathrm{BrClNaO}_{2}, \mathrm{M}+\mathrm{Na}\right]^{+}: 360.9601$, Found: 360.9609.

HPLC analysis: Chiralcel AD-H $($ Hexane $/ i$-PrOH $=80: 20$, flow rate $=1.0 \mathrm{~mL} / \mathrm{min}$, wave length $=254 \mathrm{~nm}), t_{\mathrm{R}}=9.801$ $\min$ (minor), $t_{\mathrm{R}}=11.831 \mathrm{~min}$ (major).

Optical Rotation: $[\alpha]_{\mathrm{D}}^{20}=-33.8^{\circ}\left(c=0.2, \mathrm{CHCl}_{3}\right)$.

Physical properties: white solid; Melting Point: $137.6-138.8^{\circ} \mathrm{C}$; Yield: $82 \%, 27.8 \mathrm{mg}$. 


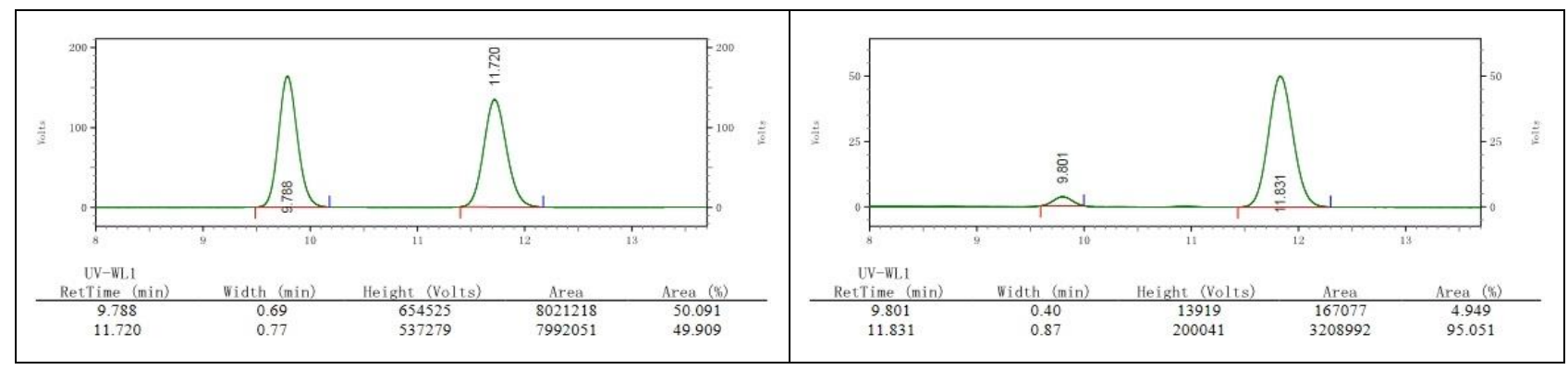

phenyl (2R,3S)-3-(4-bromophenyl)oxirane-2-carboxylate (7)<smiles>[B]c1ccc([C@@H]2O[C@H]2C(=O)Oc2ccccc2)cc1</smiles>

${ }^{1} \mathbf{H}$ NMR $\left(400 \mathrm{MHz}, \mathrm{CDCl}_{3}\right): \delta 7.51(\mathrm{~d}, J=7.7 \mathrm{~Hz}, 2 \mathrm{H}), 7.39(\mathrm{t}, J=7.0 \mathrm{~Hz}, 2 \mathrm{H}), 7.28-7.24(\mathrm{~m}$, $1 \mathrm{H}), 7.21(\mathrm{~d}, J=7.8 \mathrm{~Hz}, 2 \mathrm{H}), 7.16(\mathrm{~d}, J=7.4 \mathrm{~Hz}, 2 \mathrm{H}), 4.21(\mathrm{~d}, J=0.8 \mathrm{~Hz}, 1 \mathrm{H}), 3.68(\mathrm{~d}, J=0.8$ $\mathrm{Hz}, 1 \mathrm{H})$.

${ }^{13} \mathrm{C}$ NMR $\left(100 \mathrm{MHz}, \mathrm{CDCl}_{3}\right): \delta 166.3,150.0,133.7,131.9,129.5,127.5,126.3,123.2,121.1$,

HRMS (ESI) m/z Calcd for $\left[\mathrm{C}_{15} \mathrm{H}_{11} \mathrm{BrNaO}_{3}, \mathrm{M}+\mathrm{Na}\right]^{+}:$340.9784, Found: 340.9792.

HPLC analysis: Chiralcel OD-H (Hexane $/ i-\mathrm{PrOH}=90: 10$, flow rate $=1.0 \mathrm{~mL} / \mathrm{min}$, wave length $=210 \mathrm{~nm}), t_{\mathrm{R}}=12.663$ $\min \left(\right.$ major), $t_{\mathrm{R}}=17.605 \min$ (minor).

Optical Rotation: $[\alpha]_{\mathrm{D}}^{20}=-53.4^{\circ}\left(c=0.2, \mathrm{CHCl}_{3}\right)$.

Physical properties: white solid; Melting Point: $112.1-113.2^{\circ} \mathrm{C}$; Yield: $75 \%, 23.9 \mathrm{mg}$.

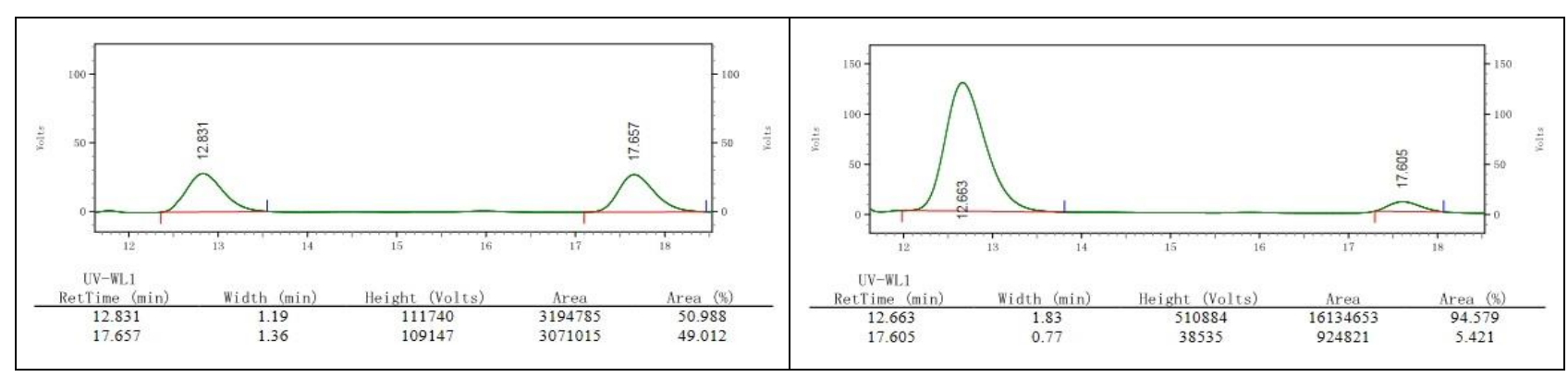




\section{Mechanistic studies}

\section{Procedure for the synthesis of deuterated $( \pm)-2 p-D$}

a) Procedure for the synthesis of 4-bromostyrene- $\beta, \beta-d_{2}$

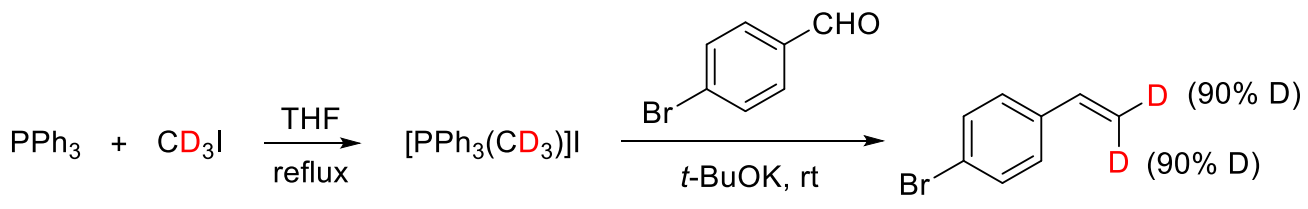

In a $100 \mathrm{~mL}$ Schlenk tube, $\mathrm{PPh}_{3}(5.25 \mathrm{~g}, 20.0 \mathrm{mmol}, 1$ equiv) was placed, evacuated and backfilled with nitrogen (3 times), THF $(30 \mathrm{~mL})$ was added. $\mathrm{CD}_{3} \mathrm{I}(1.24 \mathrm{~mL}, 20.0 \mathrm{mmol}, 1$ equiv) was added into the solution and the reaction mixture was refluxed for an hour. After cooling to room temperature, the resulting white solid was separated and was washed with $\mathrm{Et}_{2} \mathrm{O}$ and dried under reduced pressure to give $\left[\mathrm{PPh}_{3}\left(\mathrm{CD}_{3}\right)\right] \mathrm{I}(8.06 \mathrm{~g}, 19.8 \mathrm{mmol}$, $99 \%)(99 \%$ D).

$\left[\mathrm{PPh}_{3}\left(\mathrm{CD}_{3}\right)\right] \mathrm{I}\left(8.06 \mathrm{~g}, 19.8 \mathrm{mmol}, 1.2\right.$ equiv) was suspended in THF $(20 \mathrm{~mL})$ at $0{ }^{\circ} \mathrm{C}$, and $t$-BuOK $(1 \mathrm{M}, 18.1$ $\mathrm{mL}, 18.1 \mathrm{mmol}, 1.1$ equiv) was added into the reaction mixture under argon atmosphere, during which the white suspension turned to yellow suspension. After 20 minutes, rise to room temperature, 4-bromobenzaldehyde (1.7 $\mathrm{mL}, 16.5 \mathrm{mmol}, 1$ equiv) was diluted in $20 \mathrm{~mL}$ THF and slowly dropped into the solution. The reaction mixture was stirred for a night. Then water was added into the suspension and the product was extracted with EA, the organic layer was washed with brine and then dried with $\mathrm{Na}_{2} \mathrm{SO}_{4}$. The solution was concentrated under reduced pressure and the product was purified with silicagel chromatography using PE as an eluent. The product was distilled under vacuum to give 4-bromostyrene- $\beta, \beta-d_{2}(90 \% \mathrm{D})$.

b) Procedure for the synthesis of deuteriobenzaldehyde

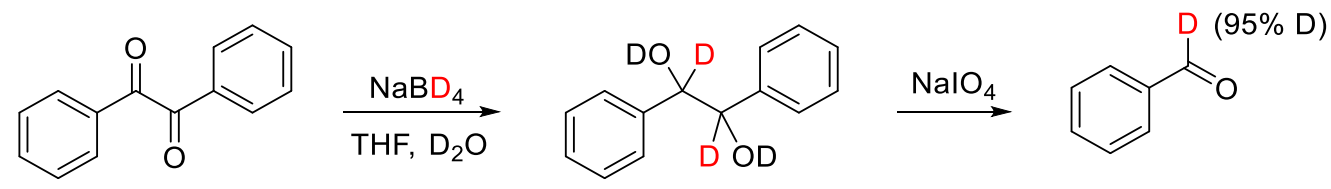

Benzil (2.1 g, $10.0 \mathrm{mmol}, 1$ equiv) was dissolved in dried THF $(20 \mathrm{~mL})$ and $\mathrm{D}_{2} \mathrm{O}(1.0 \mathrm{~mL})$ at $0{ }^{\circ} \mathrm{C}$ under argon atmosphere, $\mathrm{NaBD}_{4}(837.2 \mathrm{mg}, 20.0 \mathrm{mmol}, 2$ equiv) was added in portion-wise over one hour. Then, the reaction mixture was warm to room temperature, monitored by TLC. After general workup, $\mathrm{D}_{2} \mathrm{O}, \mathrm{NH}_{4} \mathrm{Cl}$ (dissolve in $\mathrm{D}_{2} \mathrm{O}$ ) was added into the reaction mixture, extracted with EA, dried over $\mathrm{Na}_{2} \mathrm{SO}_{4}$, filtered and concentrated to give the white solid (1.9 g, $8.8 \mathrm{mmol}, 88 \%$ ) (94\% D). The white solid was oxidized with $\mathrm{NaIO}_{4}$ in $\mathrm{CH}_{2} \mathrm{Cl}_{2}(10 \mathrm{~mL})$ and $\mathrm{D}_{2} \mathrm{O}(10 \mathrm{~mL})$ overnight at room temperature. After the reaction was complete, extracted with $\mathrm{CH}_{2} \mathrm{Cl}_{2}$, and brine, and then dried with $\mathrm{MgSO}_{4}$. The solution was concentrated under atmospheric pressure and the product was purified with silicagel chromatography $\left(\mathrm{SiO}_{2}, \mathrm{PE} / \mathrm{CH}_{2} \mathrm{Cl}_{2}=5: 1\right)(95 \% \mathrm{D})$.

c) Procedure for the synthesis of TBHP-D

$$
\gamma^{\mathrm{O}_{\mathrm{OH}}} \underset{\text { overnight, } 4 \AA}{\stackrel{n \text {-decane, } \mathrm{D}_{2} \mathrm{O}}{\longrightarrow}} \gamma^{\mathrm{O}_{\mathrm{OD}}}(90 \% \mathrm{D})
$$

This reaction was carried out according to a literature method. ${ }^{7}$ TBHP- ${ }_{1}$ was obtained by mixing TBHP in $n$ decane with 10 equiv of $\mathrm{D}_{2} \mathrm{O}$, stirring overnight and subsequent drying over molecular sieves (90\% D). 


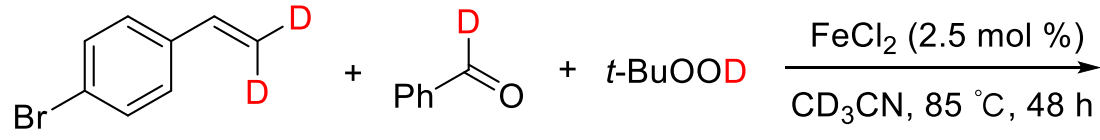<smiles>[2H]C([2H])(C(=O)c1ccccc1)[C@](OOC(C)(C)C)(c1ccccc1)c1ccc(Br)cc1</smiles>

$(85 \%$ D)

To a mixture of 4-bromostyrene- $\beta, \beta-d_{2}(0.4 \mathrm{~g}, 2.2 \mathrm{mmol}, 1$ equiv), deuteriobenzaldehyde ( $1.17 \mathrm{~g}, 10.9 \mathrm{mmol}, 5$ equiv), and $\mathrm{FeCl}_{2}\left(6.9 \mathrm{mg}, 0.05 \mathrm{mmol}, 0.025\right.$ equiv), $\mathrm{CD}_{3} \mathrm{CN}(13.0 \mathrm{~mL})$ was added under nitrogen at room temperature. Then TBHP-D $\mathrm{D}_{1}(5-6 \mathrm{M}$ in decane, $1.3 \mathrm{~mL}, 6.5 \mathrm{mmol}, 3$ equiv) was dropped into the mixture under nitrogen at room temperature. The resulting mixture was stirred under $85{ }^{\circ} \mathrm{C}$ for $48 \mathrm{~h}$. The resulting reaction solution was directly filtered through a pad of silica by dichloromethane $\left(\mathrm{SiO}_{2}, \mathrm{PE} / \mathrm{CH}_{2} \mathrm{Cl}_{2}=12: 1\right)$ to afford the product $(85 \% \mathrm{D})$.

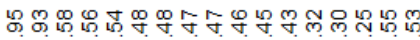

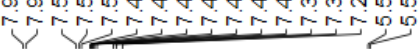

${ }^{1} \mathbf{H}$ NMR $\left(400 \mathrm{MHz}, \mathrm{CDCl}_{3}\right)$

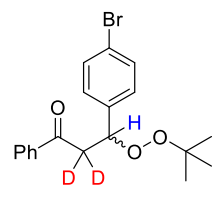

$( \pm)-1 p-D$

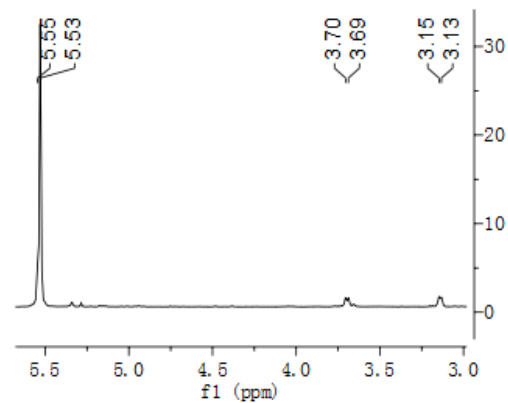

\section{$\stackrel{\infty}{i} \stackrel{6}{\frac{10}{\leftarrow}}$}

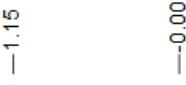

i

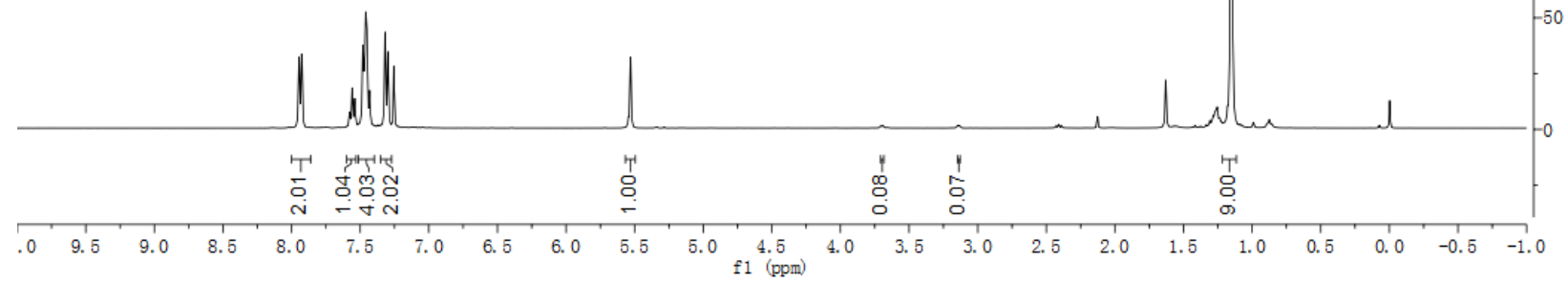




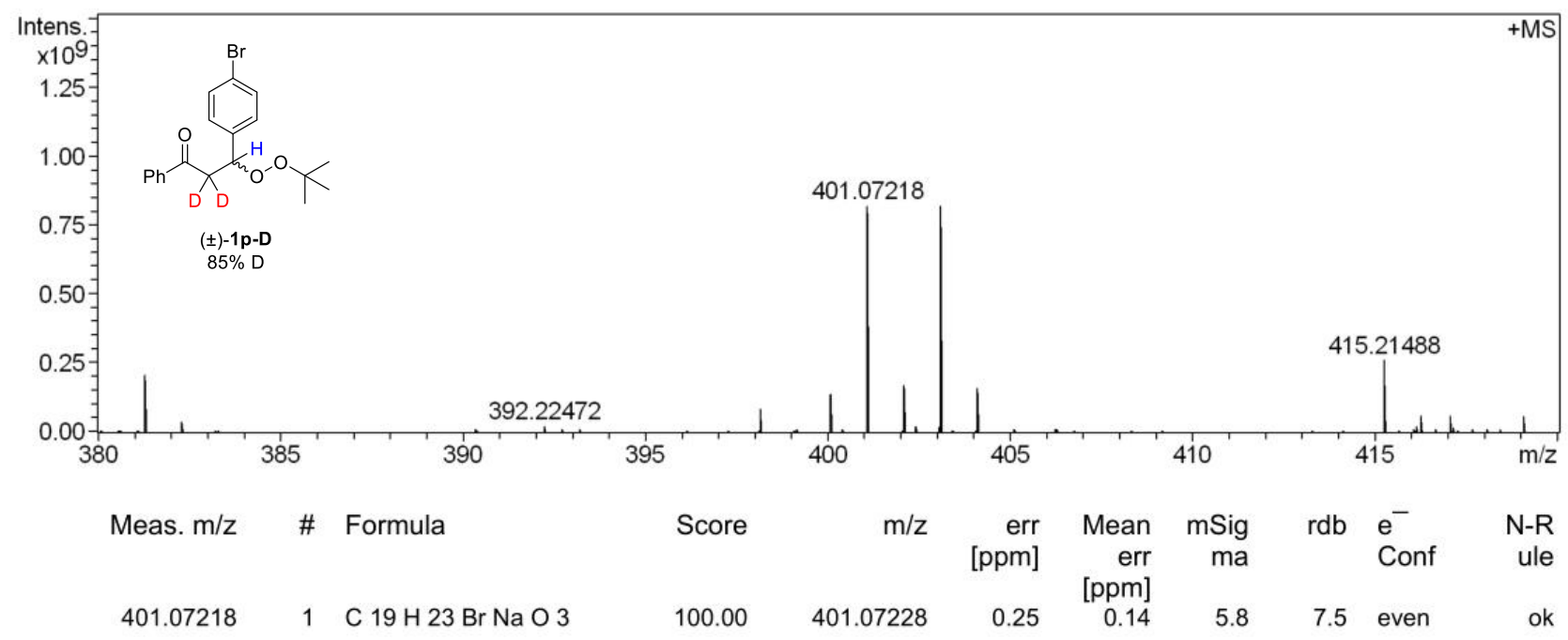

Mass Spectrum List

\begin{tabular}{|c|c|c|c|}
\hline $\mathrm{m} / \mathrm{z}$ & $\mathrm{z}$ & I & Res. \\
\hline 266.09151 & & 68427392 & 240488 \\
\hline 266.59231 & & 20424490 & 236132 \\
\hline 274.27578 & & 156969568 & 235158 \\
\hline 275.27921 & & 23131146 & 227949 \\
\hline 290.27097 & & 24546344 & 216567 \\
\hline 299.11225 & & 30578512 & 213784 \\
\hline 299.16383 & & 43258588 & 214922 \\
\hline 301.14300 & & 229047920 & 213629 \\
\hline 302.14649 & & 31931966 & 211052 \\
\hline 302.30737 & & 145285984 & 212742 \\
\hline 303.31082 & & 24337182 & 206069 \\
\hline 304.26319 & & 64617424 & 209690 \\
\hline 305.15920 & & 22382838 & 206588 \\
\hline 318.30245 & & 169017776 & 201450 \\
\hline 319.30594 & & 26224190 & 199678 \\
\hline 330.33896 & & 151739536 & 194032 \\
\hline 331.34249 & & 27336230 & 190332 \\
\hline
\end{tabular}




\begin{tabular}{|c|c|c|}
\hline 346.33417 & 58403716 & 183542 \\
\hline 349.18599 & 21535944 & 181888 \\
\hline 353.26651 & 22475058 & 418781 \\
\hline 353.26875 & 313229632 & 180993 \\
\hline 353.27101 & 22641970 & 342380 \\
\hline 354.27235 & 53931536 & 179364 \\
\hline 356.35504 & 33772008 & 178292 \\
\hline 358.37075 & 52465596 & 179182 \\
\hline 360.32649 & 55683980 & 177482 \\
\hline 362.32931 & 32182386 & 174109 \\
\hline 374.36582 & 59015124 & 171063 \\
\hline 381.30050 & 208131504 & 168130 \\
\hline 382.30404 & 41245912 & 164914 \\
\hline 392.22472 & 23163140 & 162947 \\
\hline 398.12435 & 85139096 & 159851 \\
\hline 400.06615 & 139076896 & 159225 \\
\hline 401.06776 & 27689572 & 310731 \\
\hline 401.06929 & 58230372 & 371816 \\
\hline 401.07218 & 820840064 & 159299 \\
\hline 401.07509 & 57425512 & 305716 \\
\hline 401.07666 & 25734758 & 333874 \\
\hline 402.06417 & 1451009936 & 158617 \\
\hline 402.07574 & 172898224 & 158160 \\
\hline 402.39757 & 21312794 & 153672 \\
\hline 403.06570 & 27042038 & 299183 \\
\hline 403.06728 & 60827896 & 363943 \\
\hline 403.07022 & 820168960 & 159259 \\
\hline 403.07313 & 59504888 & 378092 \\
\hline 403.07476 & 26169594 & 349679 \\
\hline & S34 & \\
\hline
\end{tabular}




\begin{tabular}{|c|c|c|}
\hline 404.07375 & 159900224 & 157645 \\
\hline 415.21488 & 263899152 & 154207 \\
\hline 416.12655 & 25146920 & 156089 \\
\hline 416.21845 & 62232900 & 154410 \\
\hline 417.04670 & 57879612 & 152893 \\
\hline 417.12540 & 19978066 & 146937 \\
\hline 419.04466 & 61906560 & 154362 \\
\hline 432.23831 & 30610826 & 261534 \\
\hline 432.24164 & 383932800 & 148622 \\
\hline 432.24499 & 29223308 & 362841 \\
\hline 433.24531 & 90882648 & 148832 \\
\hline 434.18889 & 69050136 & 149767 \\
\hline 434.69057 & 32710010 & 150157 \\
\hline 437.18783 & 21605700 & 332050 \\
\hline 437.18966 & 34428228 & 325665 \\
\hline 437.19150 & 63839556 & 293527 \\
\hline 437.19354 & 195808576 & 288719 \\
\hline 437.19687 & 1940368768 & 149180 \\
\hline 437.20027 & 153218368 & 372327 \\
\hline 437.20217 & 61103428 & 355683 \\
\hline 437.20403 & 34399560 & 345719 \\
\hline 437.20587 & 20755782 & 356274 \\
\hline 438.19714 & 43259372 & 276602 \\
\hline 438.20047 & 505802208 & 147008 \\
\hline 438.20393 & 36513264 & 364628 \\
\hline 439.20430 & 54653584 & 163259 \\
\hline 453.16761 & 22840350 & 237517 \\
\hline 453.17126 & 217437216 & 144080 \\
\hline 454.17502 & 49251928 & 143472 \\
\hline
\end{tabular}




\begin{tabular}{lll}
460.27358 & 51031924 & 142359 \\
474.28554 & 21048234 & 224002 \\
474.28941 & 183340976 & 138032 \\
475.29292 & 51356132 & 138566 \\
479.08776 & 30643936 & 137507 \\
481.08593 & 30128004 & 138452 \\
488.30555 & 34866364 & 133566 \\
512.50896 & 21355260 & 123954 \\
598.32293 & 63873200 & 107307 \\
599.32640 & 22456764 & 112213 \\
\hline
\end{tabular}

\section{Kinetic isotopic effect study}

$$
\mathrm{KIE}=K_{H} / K_{D}=2.88
$$<smiles>CC(C)(C)OOC(C(=O)c1ccccc1)c1ccc(Br)cc1</smiles>

$( \pm)-1 p$

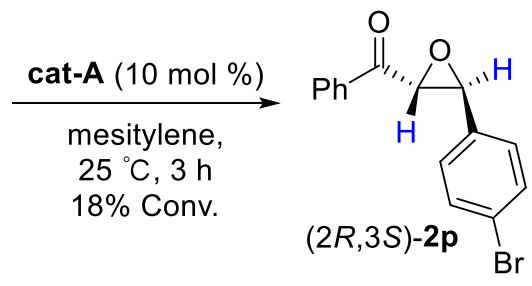

Independent Reactions<smiles>[2H]C([2H])(C(=O)c1ccccc1)C(OOC(C)(C)C)(c1ccccc1)c1ccc(Br)cc1</smiles>

$( \pm)-1 p-D$

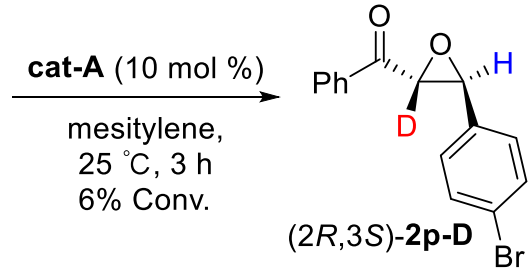

Two parallel reactions with $( \pm)-\mathbf{1 p}$ and $( \pm)-\mathbf{1} \mathbf{p}-\mathbf{D}$ were ran at same time. $( \pm)-\mathbf{1 p}(0.1 \mathrm{mmol})$ or $( \pm)-\mathbf{1 p}-\mathbf{D}(0.1 \mathrm{mmol})$ and cat-A (5.9 mg, $0.01 \mathrm{mmol}, 0.1$ equiv) was added to a $10 \mathrm{~mL}$ flame-dried Schlenk tube with a magnetic stirring bar. Then, mesitylene $(1.5 \mathrm{~mL})$ was injected into the tube. After stirring for $3 \mathrm{~h}$. The resulting mixture was dried under reduced pressure, and then sent for ${ }^{1} \mathrm{H}$ NMR testing immediately. The KIE $\left(k_{\mathrm{H}} / k_{\mathrm{D}}\right)$ value was calculated according to the ${ }^{1} \mathrm{H}$ NMR spectrum. 
(I)-1p
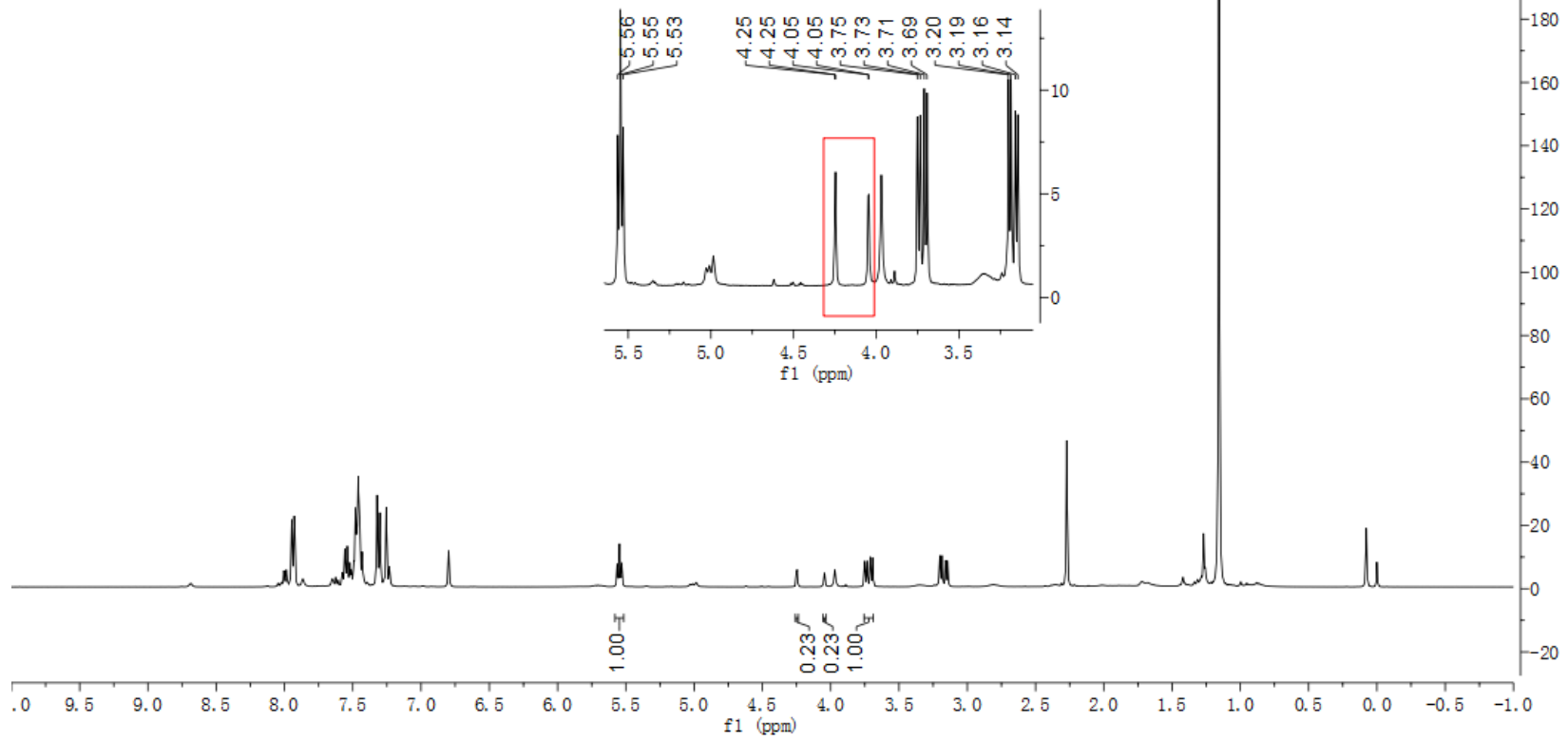

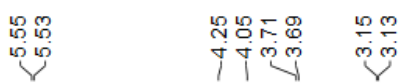

(I)-1p-D
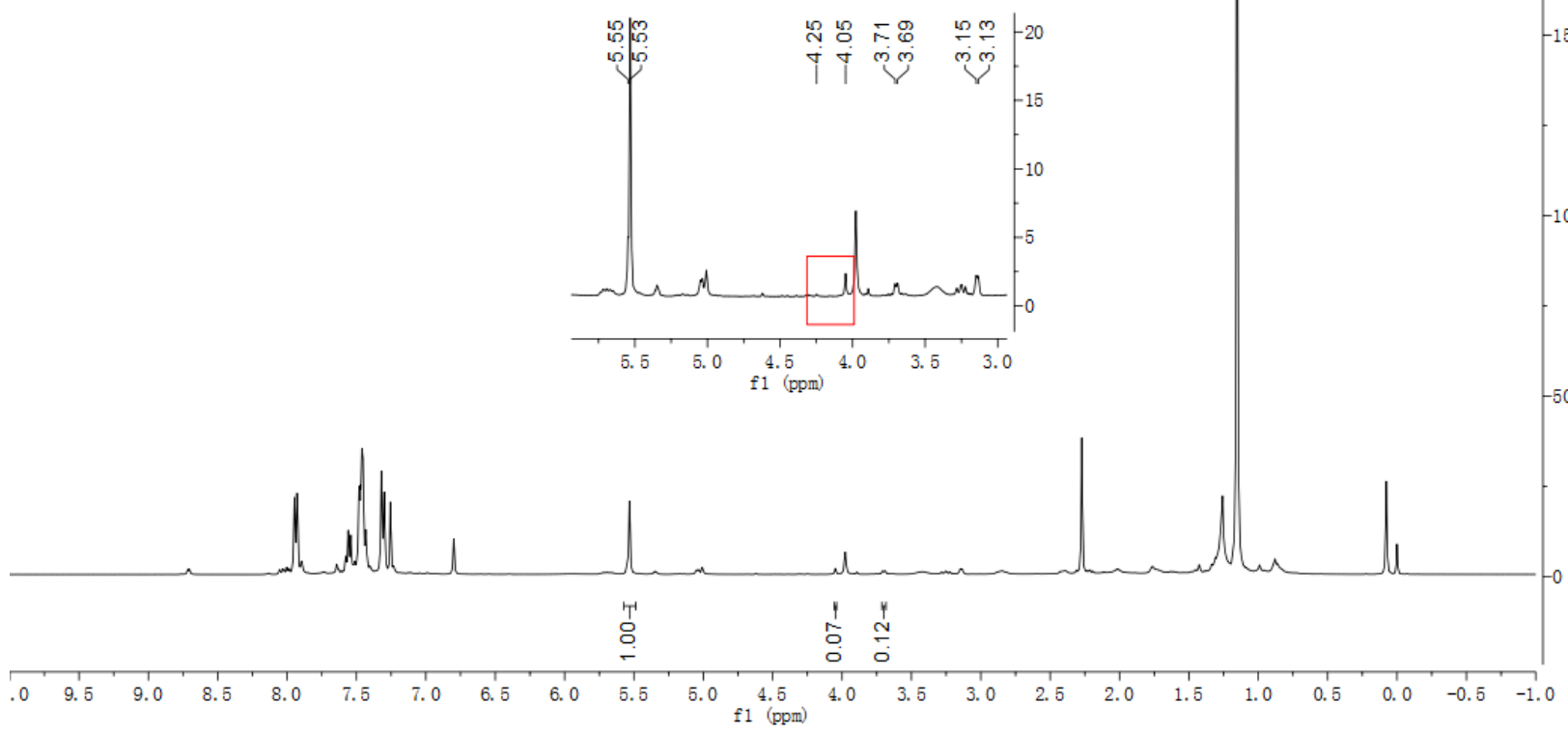


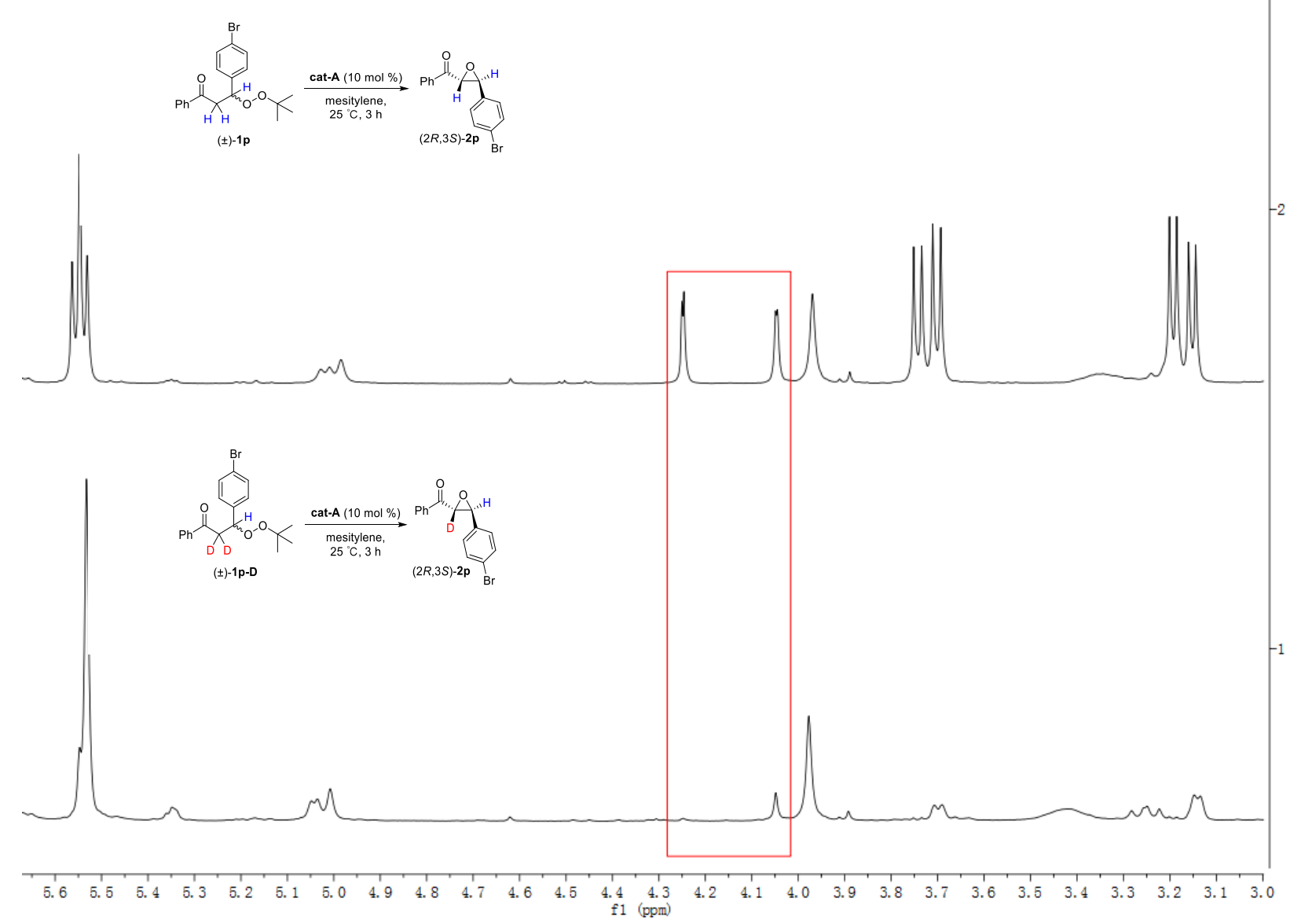


IX. ${ }^{1} \mathrm{H}$ and ${ }^{13} \mathrm{C}$ NMR spectra of compounds (1a-s, 2a-s)

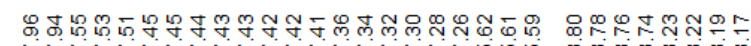

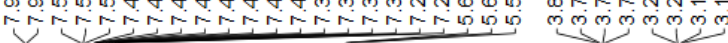

$\stackrel{0}{i} \quad \stackrel{8}{i}$

${ }^{1} \mathbf{H}$ NMR $\left(400 \mathrm{MHz}, \mathrm{CDCl}_{3}\right)$<smiles>CC(C)(C)OOC(CC(=O)c1ccccc1)c1ccccc1</smiles>

(S)-1a

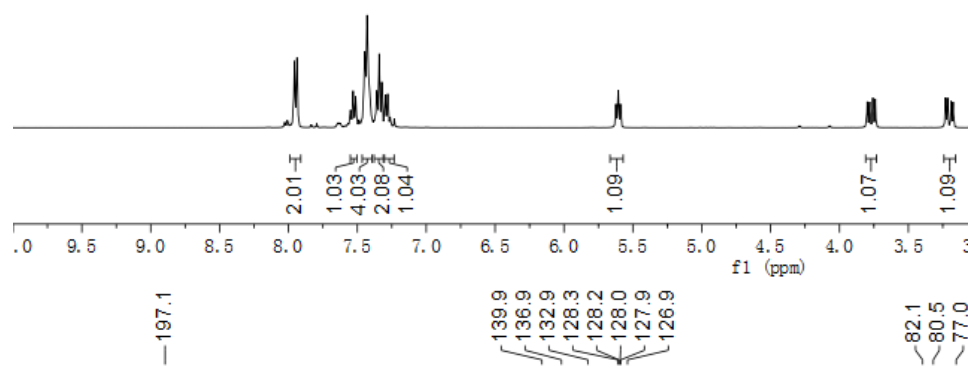

${ }^{13} \mathbf{C}$ NMR $\left(100 \mathrm{MHz}, \mathrm{CDCl}_{3}\right)$<smiles>CC(C)(C)OOC(CC(=O)c1ccccc1)c1ccccc1</smiles>

$(S)-1 \mathbf{a}$
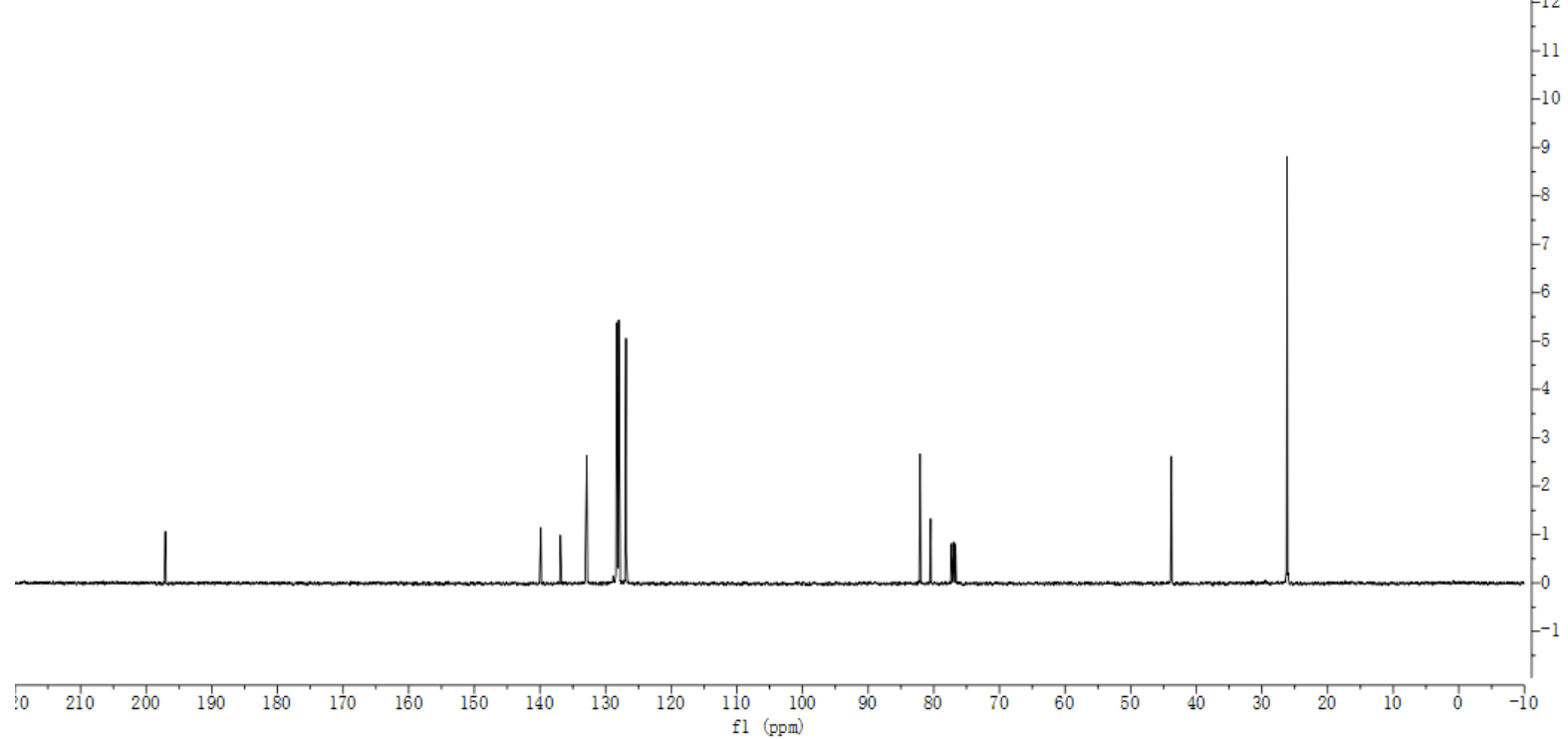


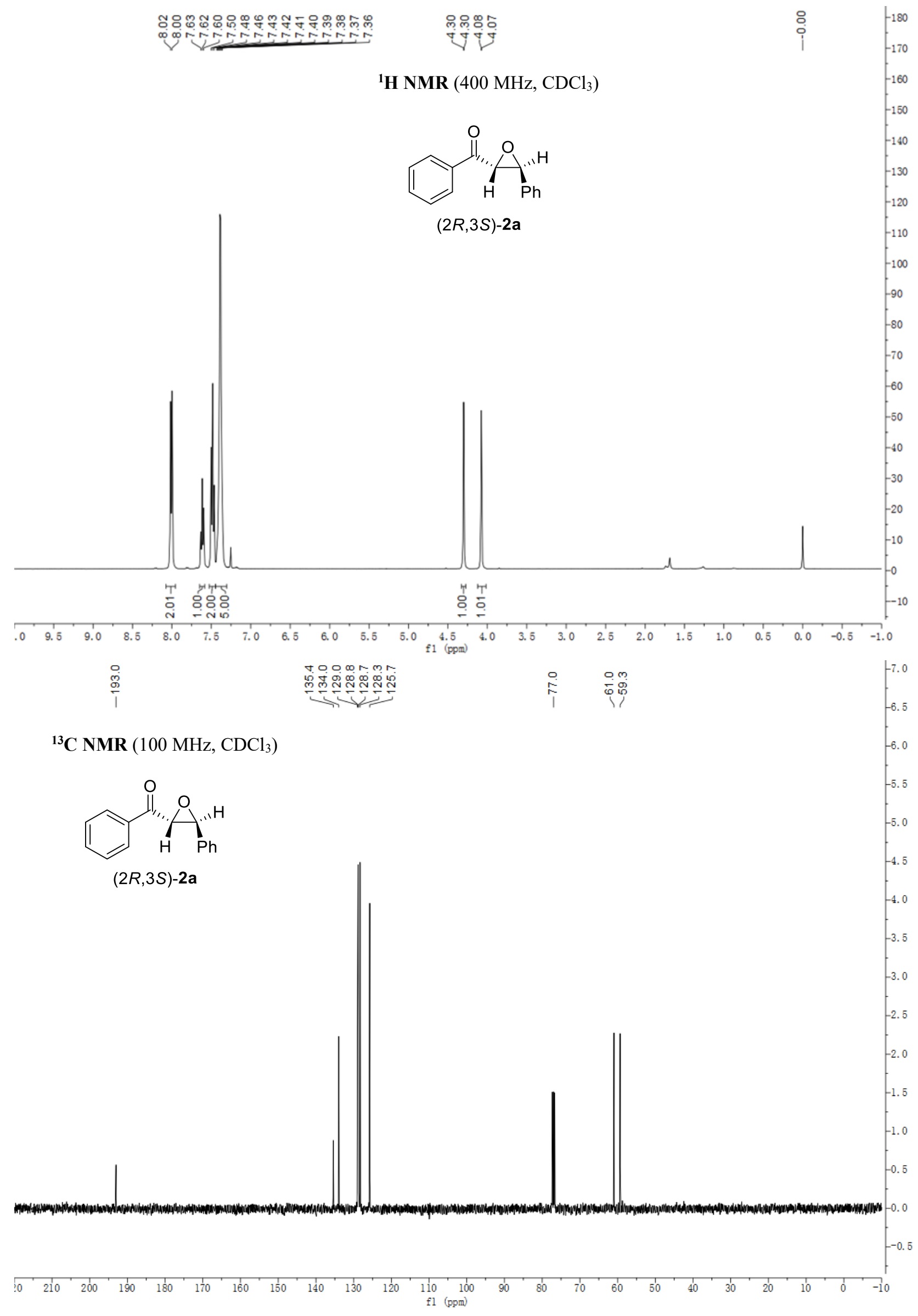


${ }^{1} \mathbf{H}$ NMR $\left(400 \mathrm{MHz}, \mathrm{CDCl}_{3}\right)$<smiles>Cc1ccc(C(=O)CC(OOC(C)(C)C)c2ccccc2)cc1</smiles>

(S)-1 $\mathbf{b}$

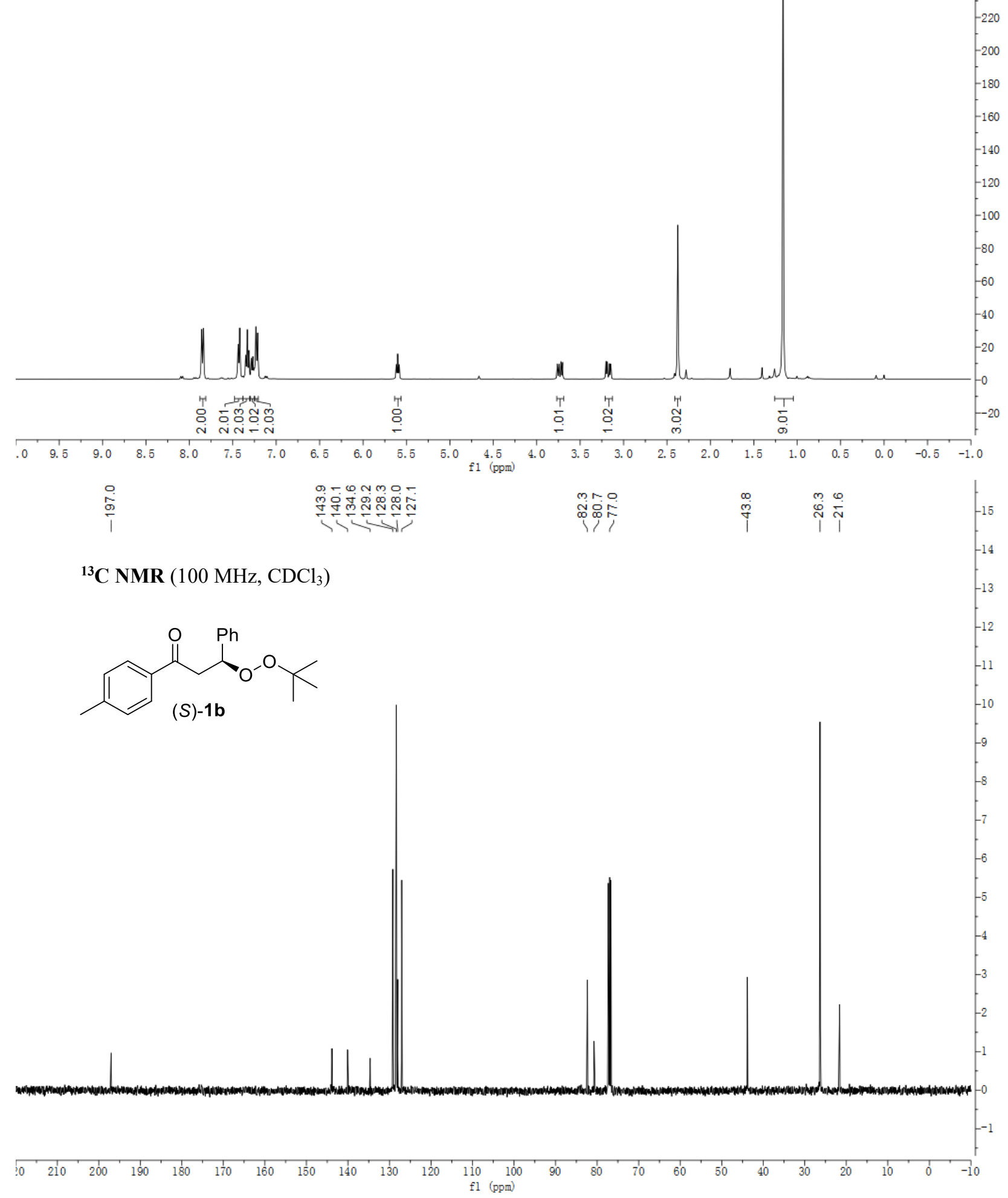




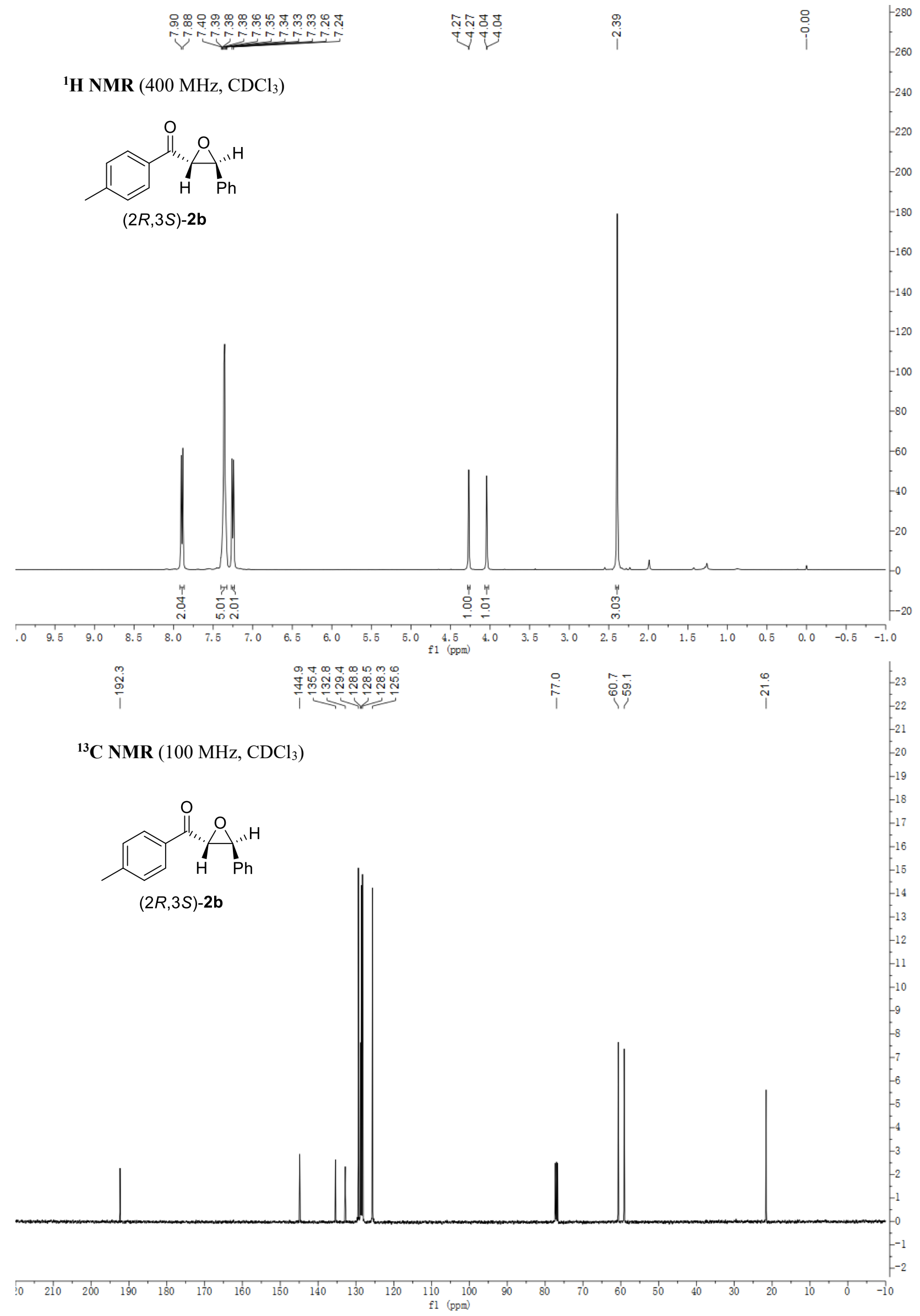




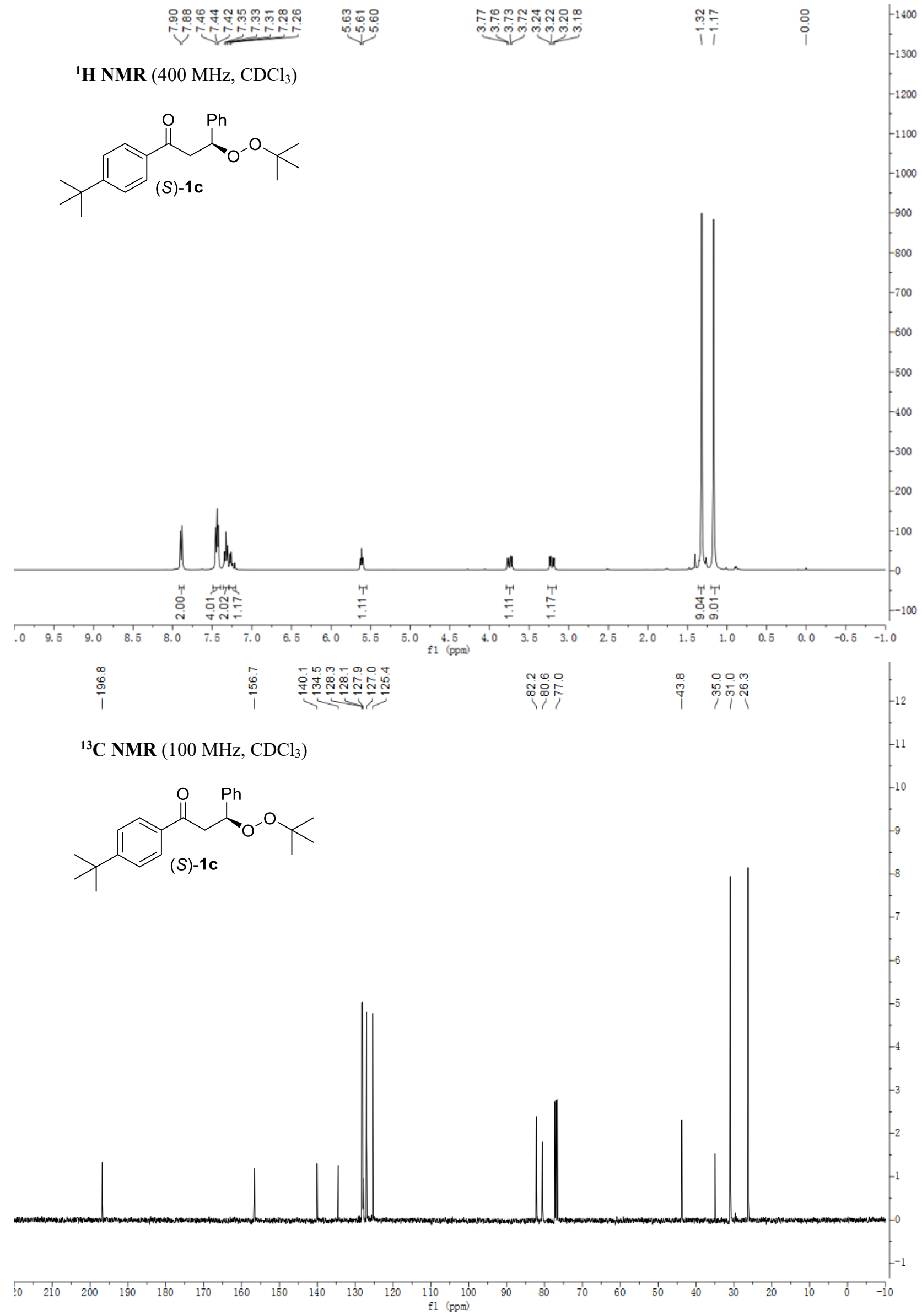


${ }^{1} \mathbf{H}$ NMR (400 MHz, $\left.\mathrm{CDCl}_{3}\right)$

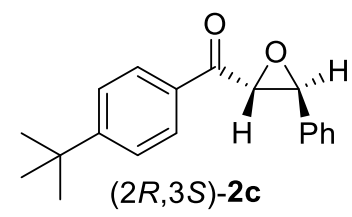

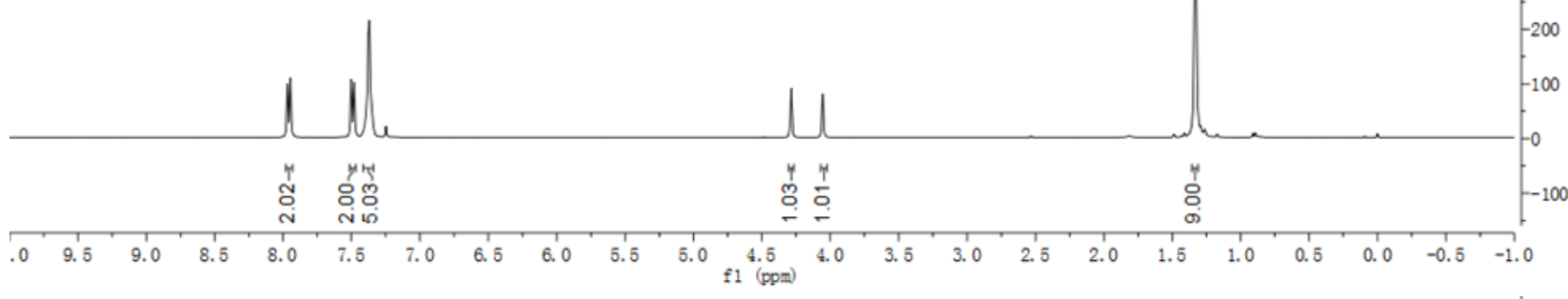

管

${ }^{13} \mathbf{C}$ NMR $\left(100 \mathrm{MHz}, \mathrm{CDCl}_{3}\right)$<smiles>[R16]c1ccc(C(=O)[C@@H]2O[C@H]2c2ccc(C(C)(C)C)cc2)cc1</smiles>

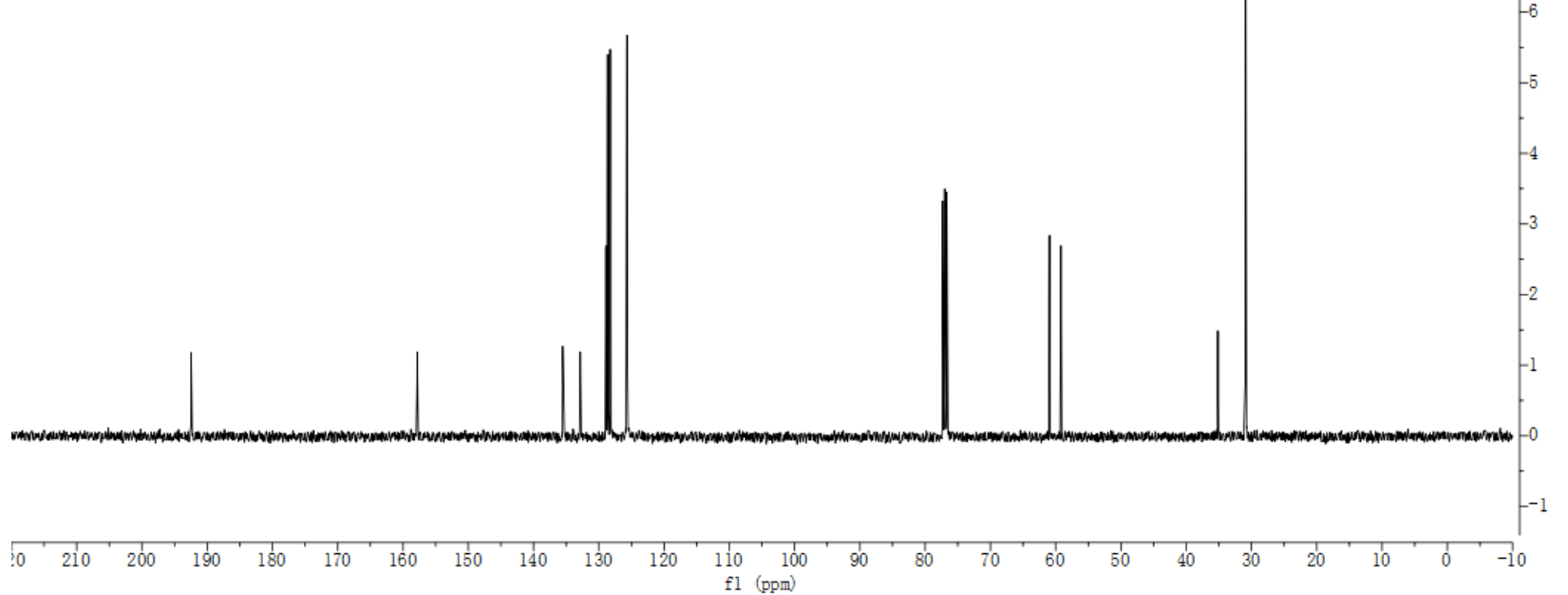


${ }^{1} \mathbf{H}$ NMR $\left(400 \mathrm{MHz}, \mathrm{CDCl}_{3}\right)$<smiles>Cc1ccc(C(=O)CC(OOC(C)(C)C)c2ccccc2)cc1C</smiles>

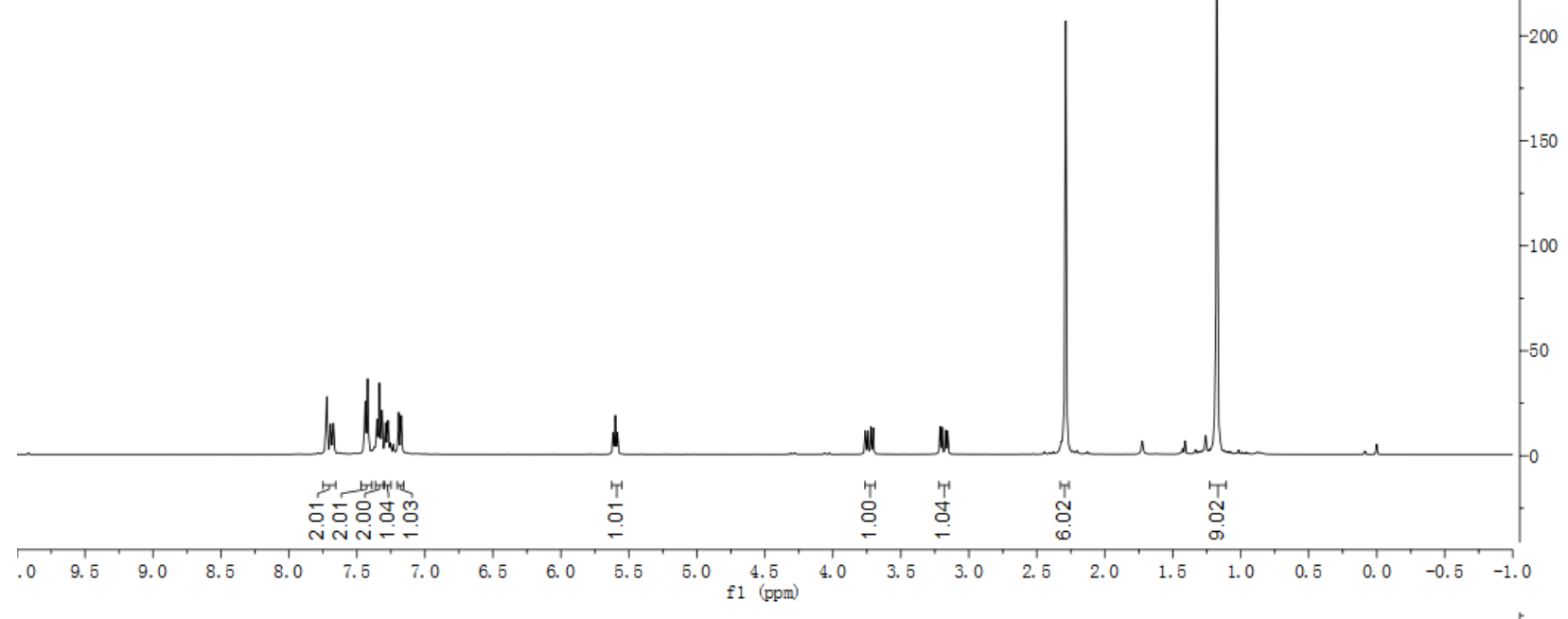

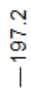

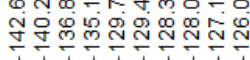

min

Nof

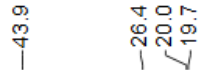

${ }^{13} \mathbf{C}$ NMR $\left(100 \mathrm{MHz}, \mathrm{CDCl}_{3}\right)$<smiles>Cc1ccc(C(=O)CC(OOC(C)(C)C)c2ccccc2)cc1C</smiles>

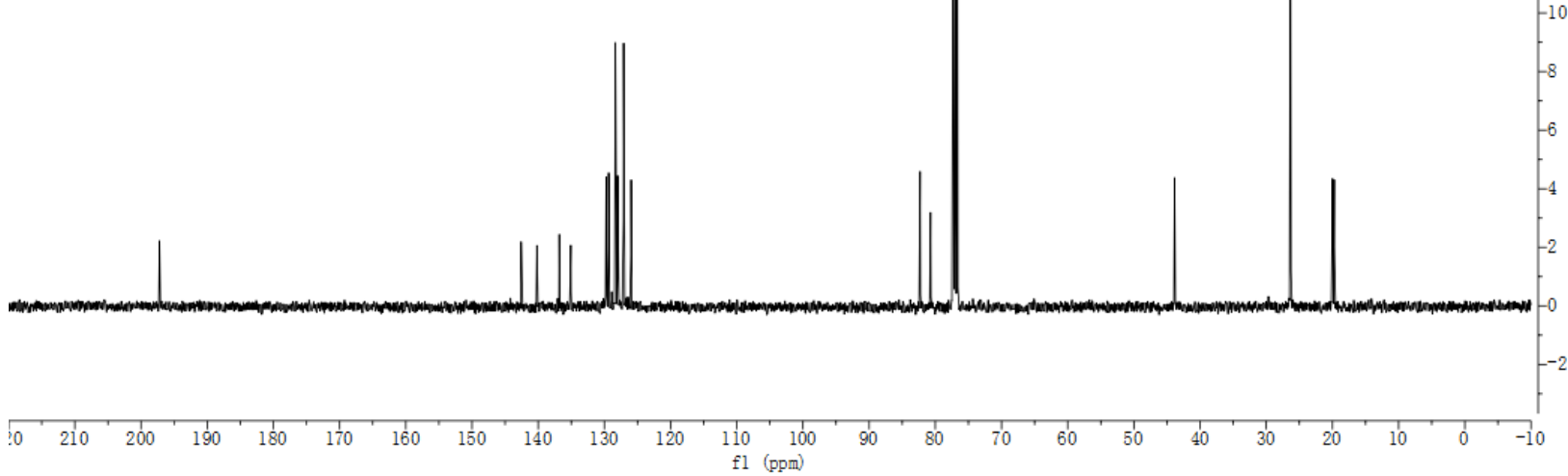




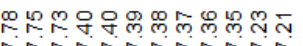

Mintis

我

过艺

ํํำ

$\stackrel{-1200}{i} \quad-1$

${ }^{1} \mathbf{H}$ NMR $\left(400 \mathrm{MHz}, \mathrm{CDCl}_{3}\right)$

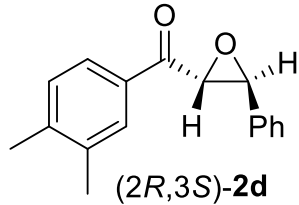

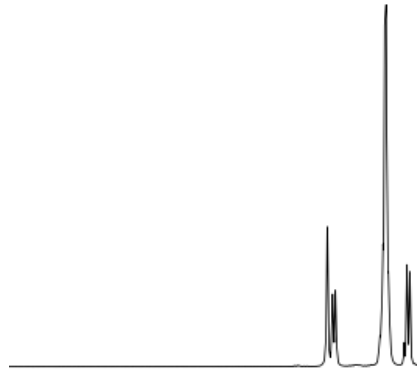

党

๙ั)

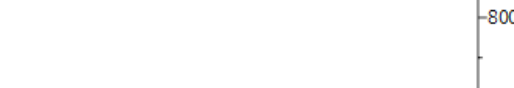

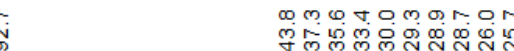

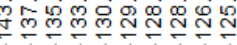

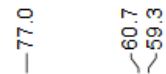

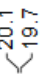

${ }^{13}$ C NMR (100 MHz, $\left.\mathrm{CDCl}_{3}\right)$<smiles>Cc1ccc(C(=O)[C@H]2O[C@H]2c2ccccc2)cc1C</smiles>

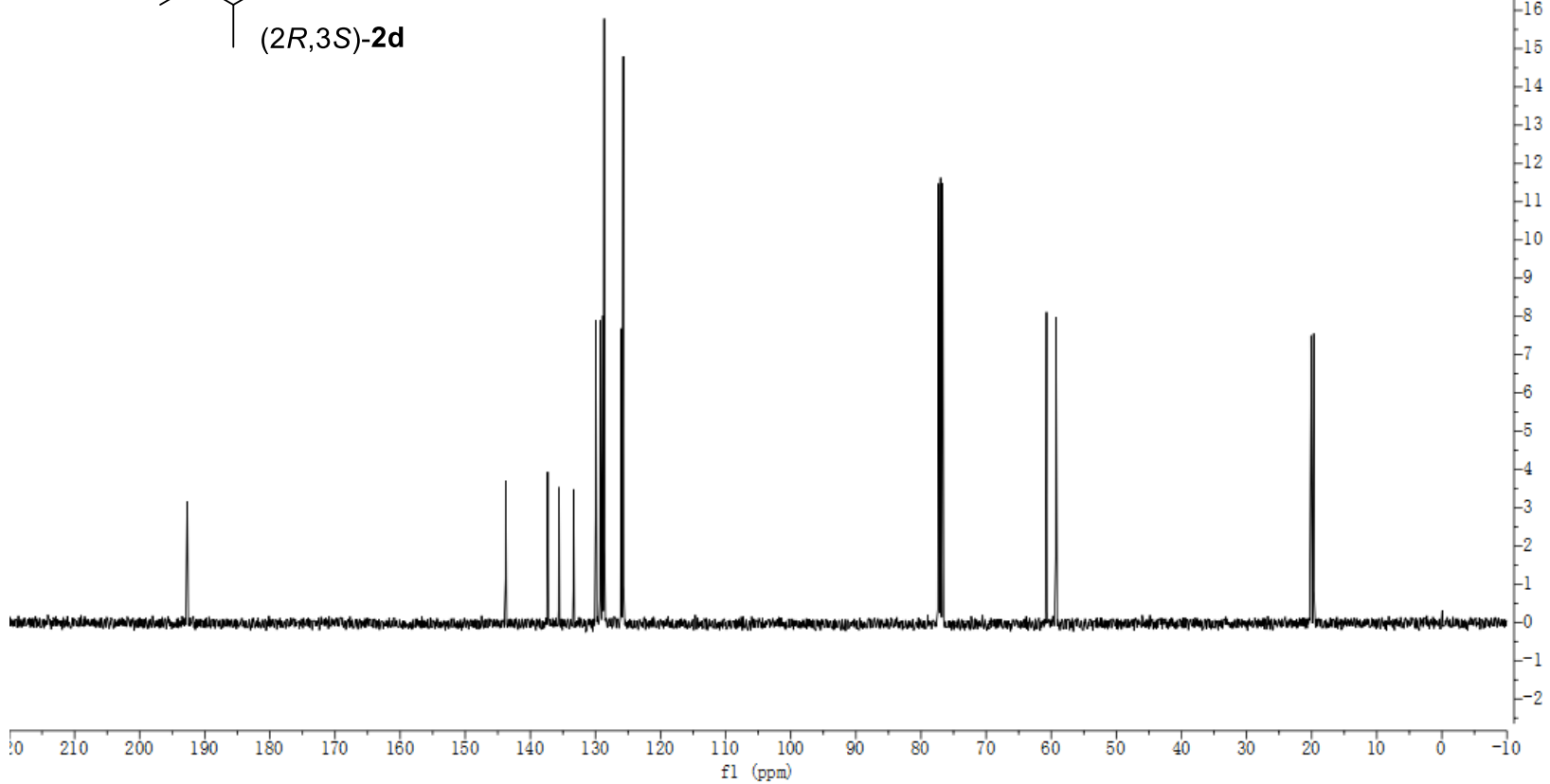


${ }^{1} \mathbf{H}$ NMR $\left(400 \mathrm{MHz}, \mathrm{CDCl}_{3}\right)$<smiles>COc1cccc(C(=O)CC(OOC(C)(C)C)c2ccccc2)c1</smiles>

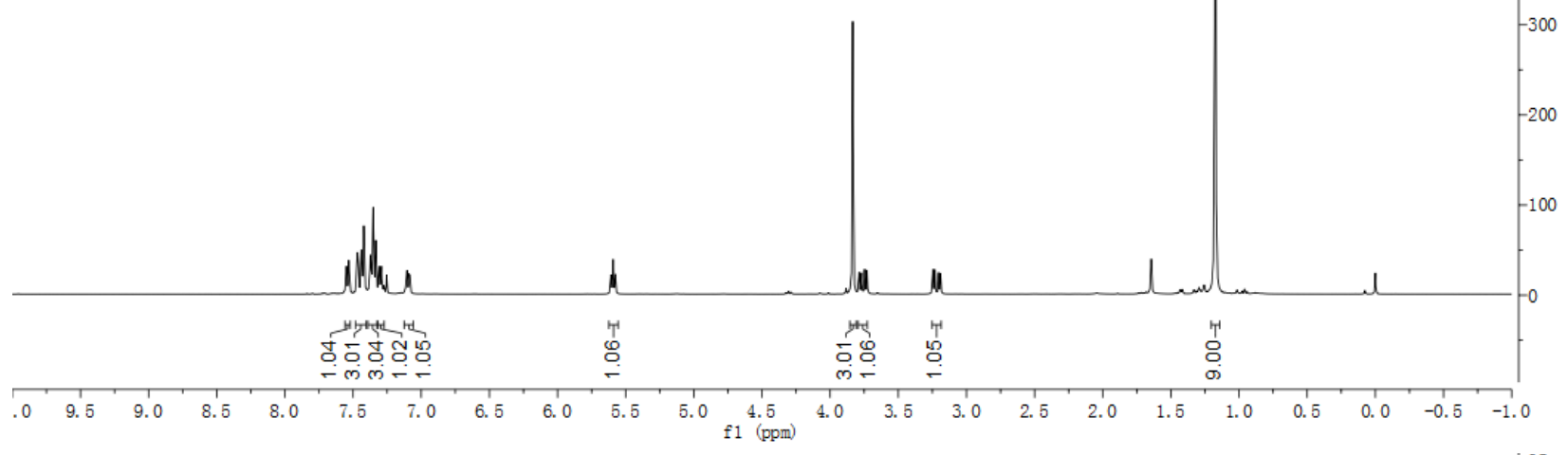

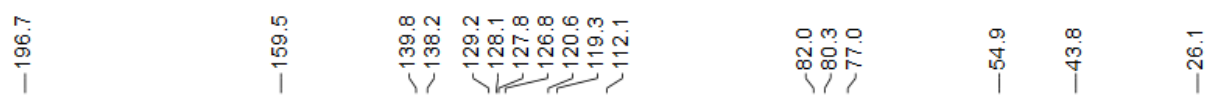

${ }^{13} \mathbf{C}$ NMR $\left(100 \mathrm{MHz}, \mathrm{CDCl}_{3}\right)$<smiles>COc1cccc(C(=O)CC(OOC(C)(C)C)c2ccccc2)c1</smiles>

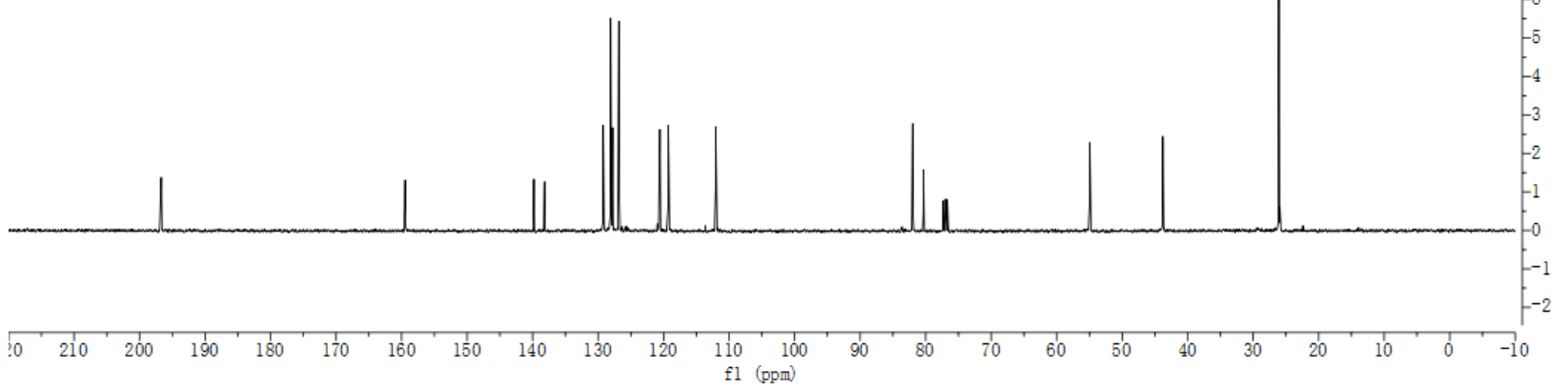


${ }^{1} \mathbf{H}$ NMR $\left(400 \mathrm{MHz}, \mathrm{CDCl}_{3}\right)$<smiles>COc1cccc(C(=O)[C@@H]2O[C@H]2c2ccccc2)c1</smiles>

$(2 R, 3 S)-2 \mathrm{e}$

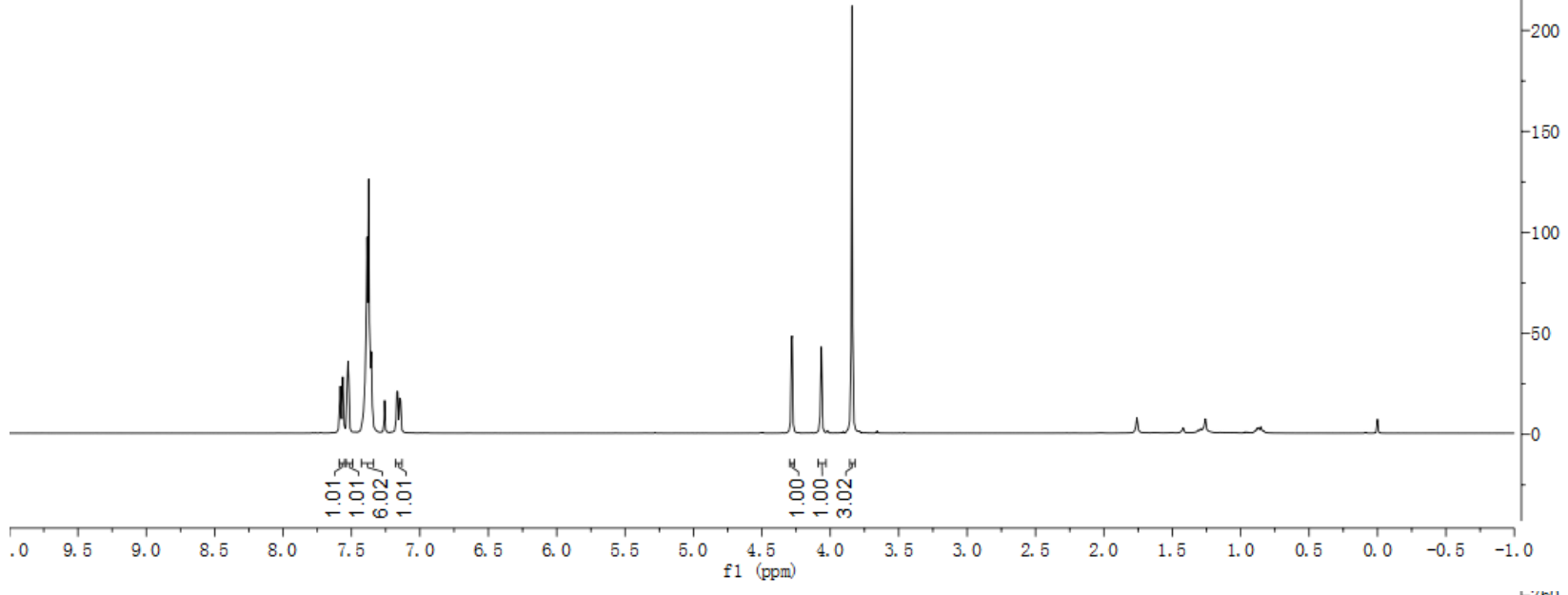

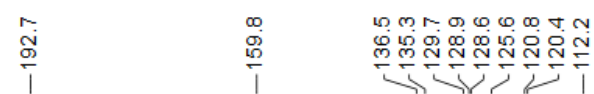

${ }^{13} \mathbf{C}$ NMR $\left(100 \mathrm{MHz}, \mathrm{CDCl}_{3}\right)$

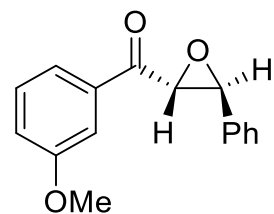

$(2 R, 3 S)-2 \mathbf{e}$

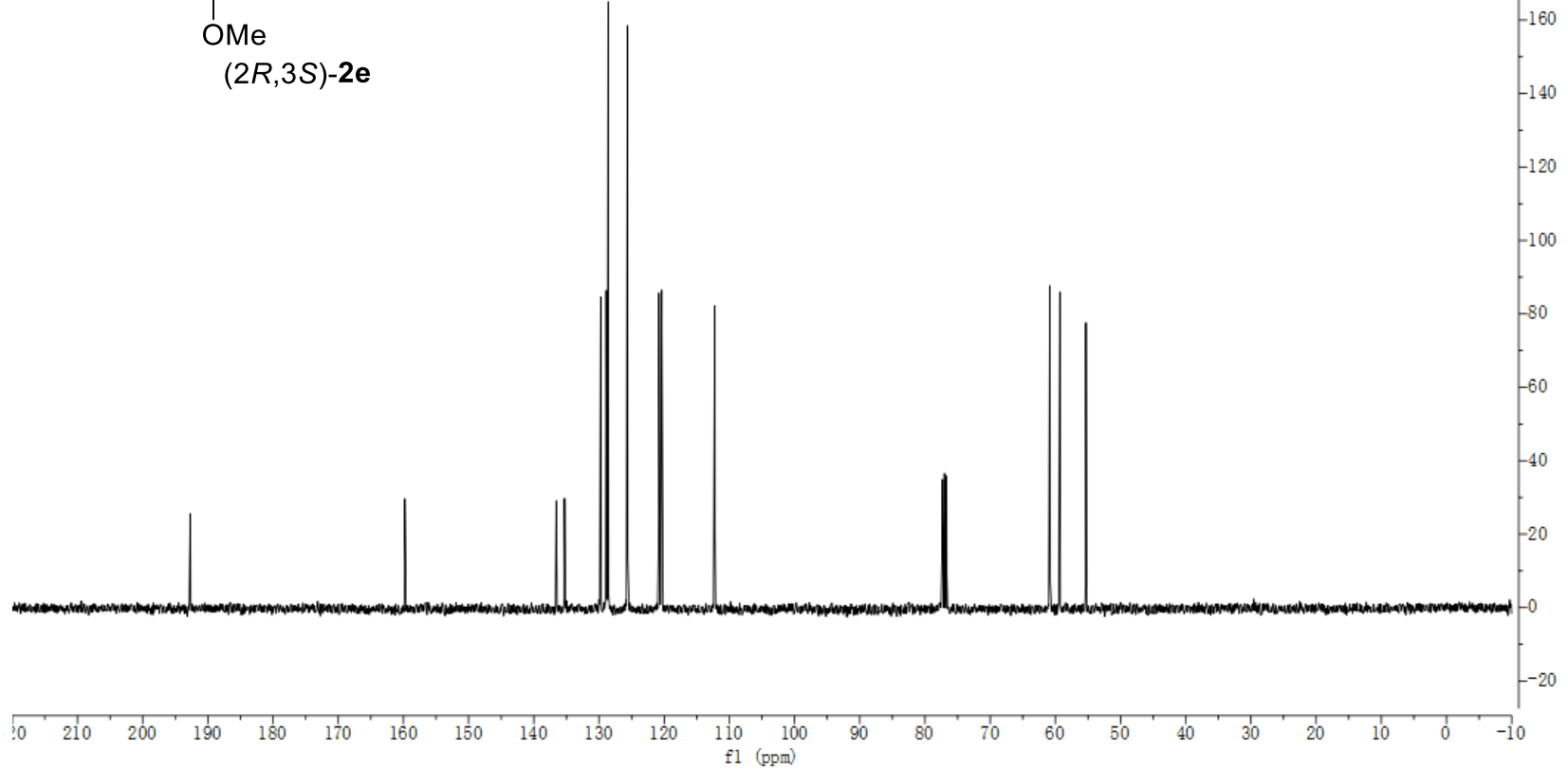


${ }^{1} \mathbf{H}$ NMR $\left(400 \mathrm{MHz}, \mathrm{CDCl}_{3}\right)$<smiles>COc1cc(C(=O)CC(OOC(C)(C)C)c2ccccc2)cc(OC)c1OC</smiles>

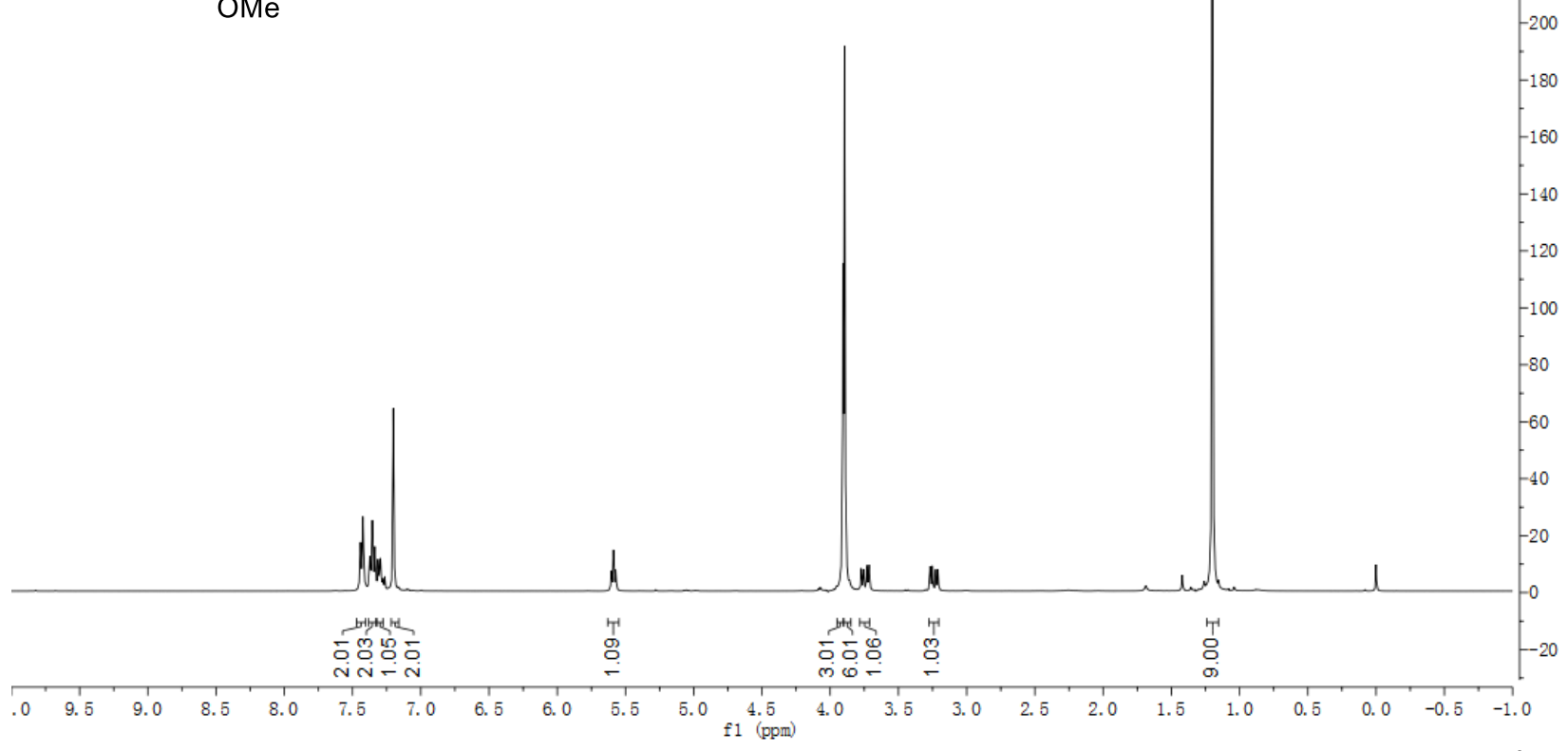

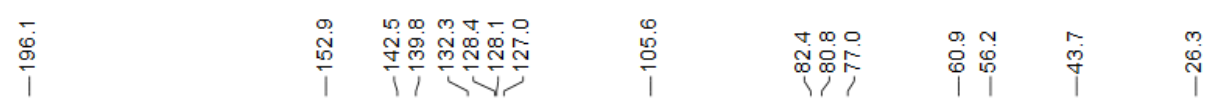

${ }^{13} \mathbf{C}$ NMR $\left(100 \mathrm{MHz}, \mathrm{CDCl}_{3}\right)$<smiles>COc1cc(C(=O)CC(OOC(C)(C)C)c2ccccc2)cc(OC)c1OC</smiles>

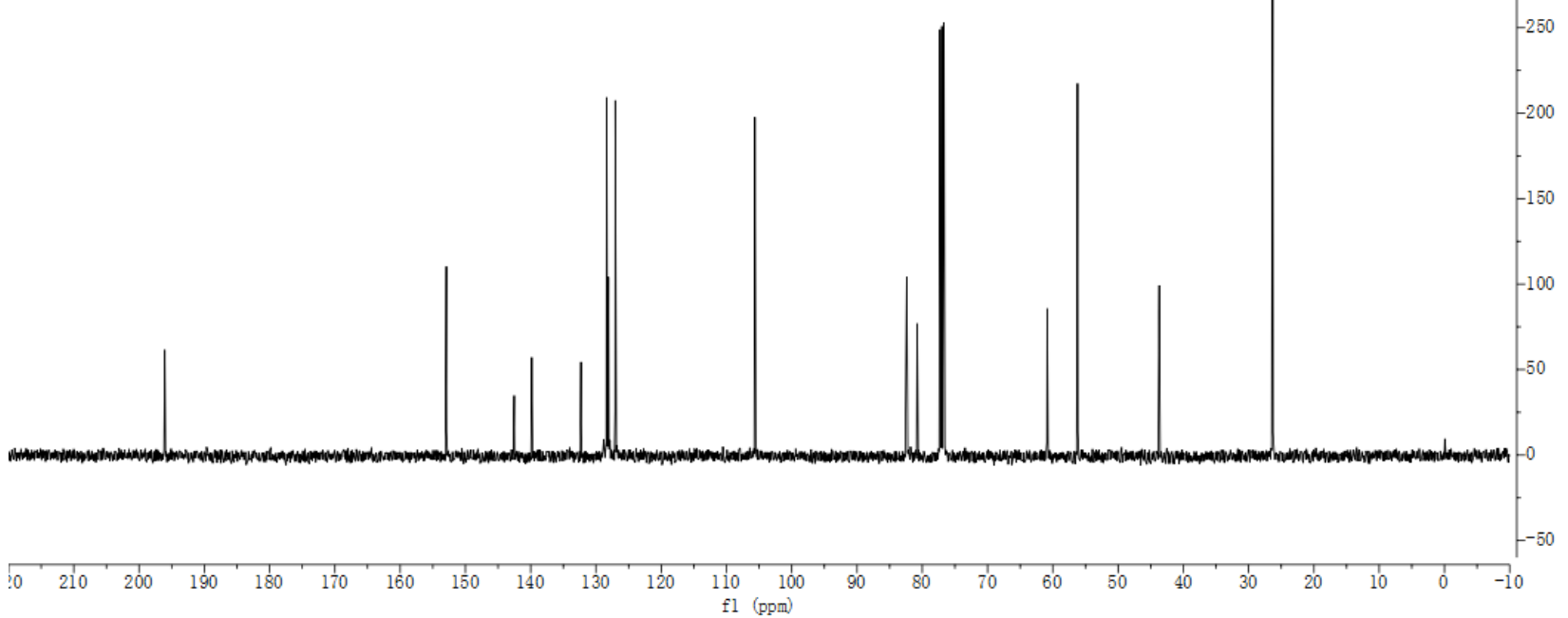




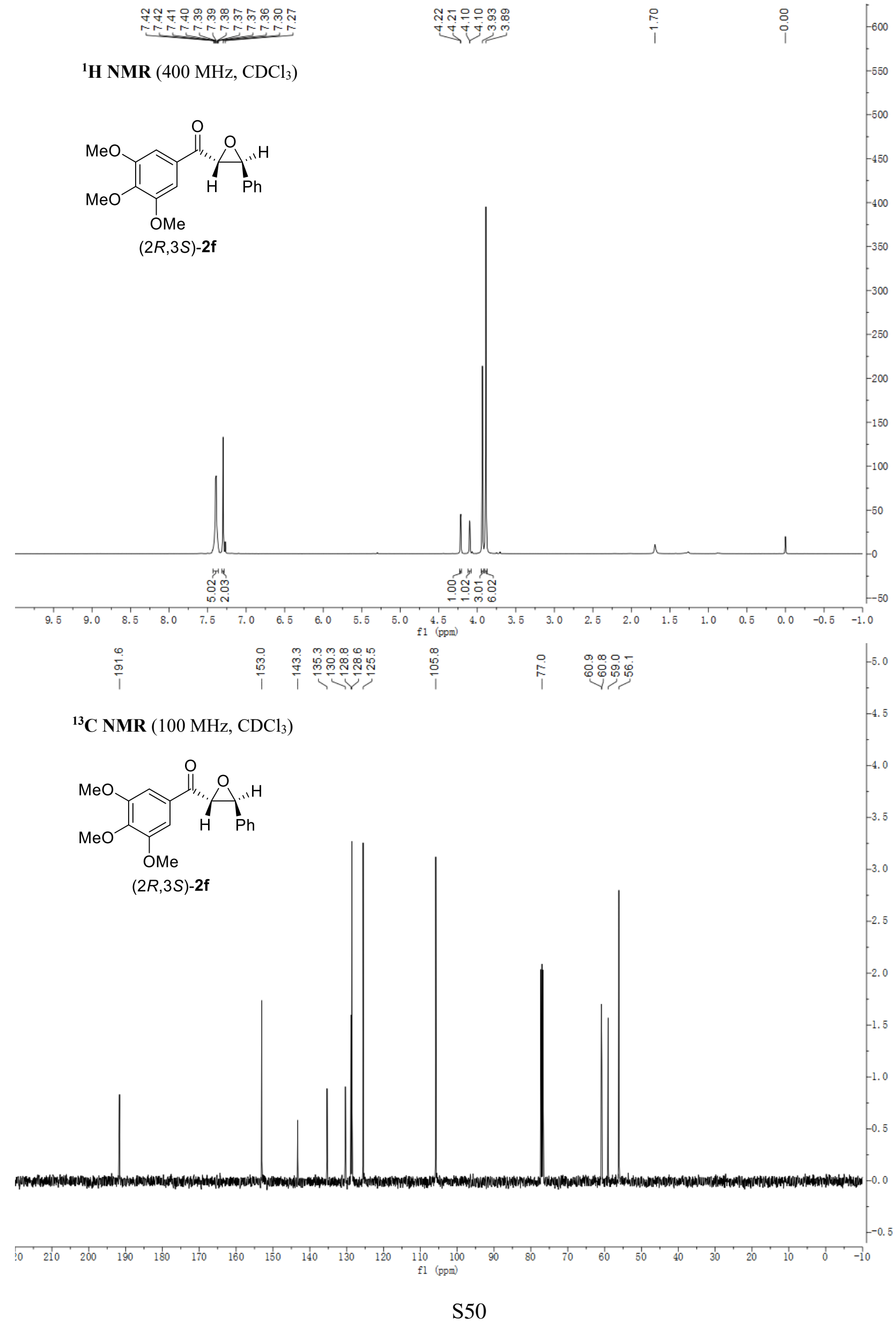


${ }^{1} \mathbf{H}$ NMR (400 MHz, $\left.\mathrm{CDCl}_{3}\right)$<smiles>CC(C)(C)OOC(CC(=O)c1ccc(Cl)cc1)c1ccccc1</smiles>

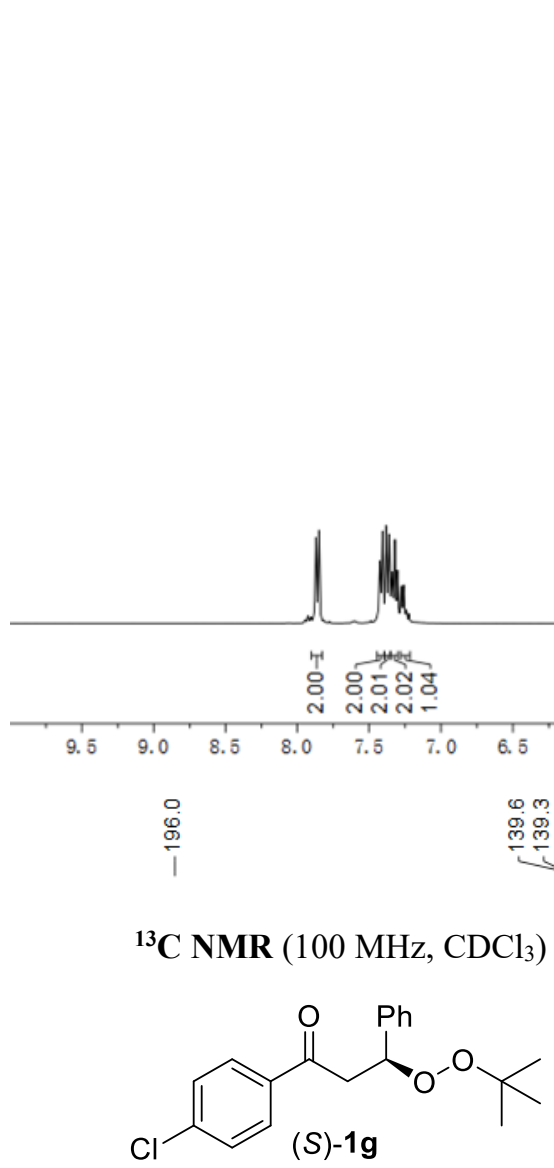

${ }^{13} \mathbf{C}$ NMR $\left(100 \mathrm{MHz}, \mathrm{CDCl}_{3}\right)$ m m क 兽
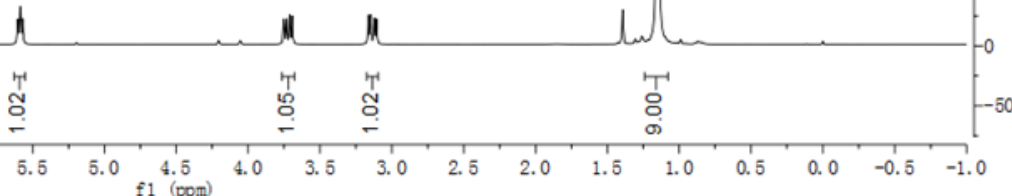

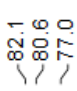

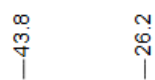
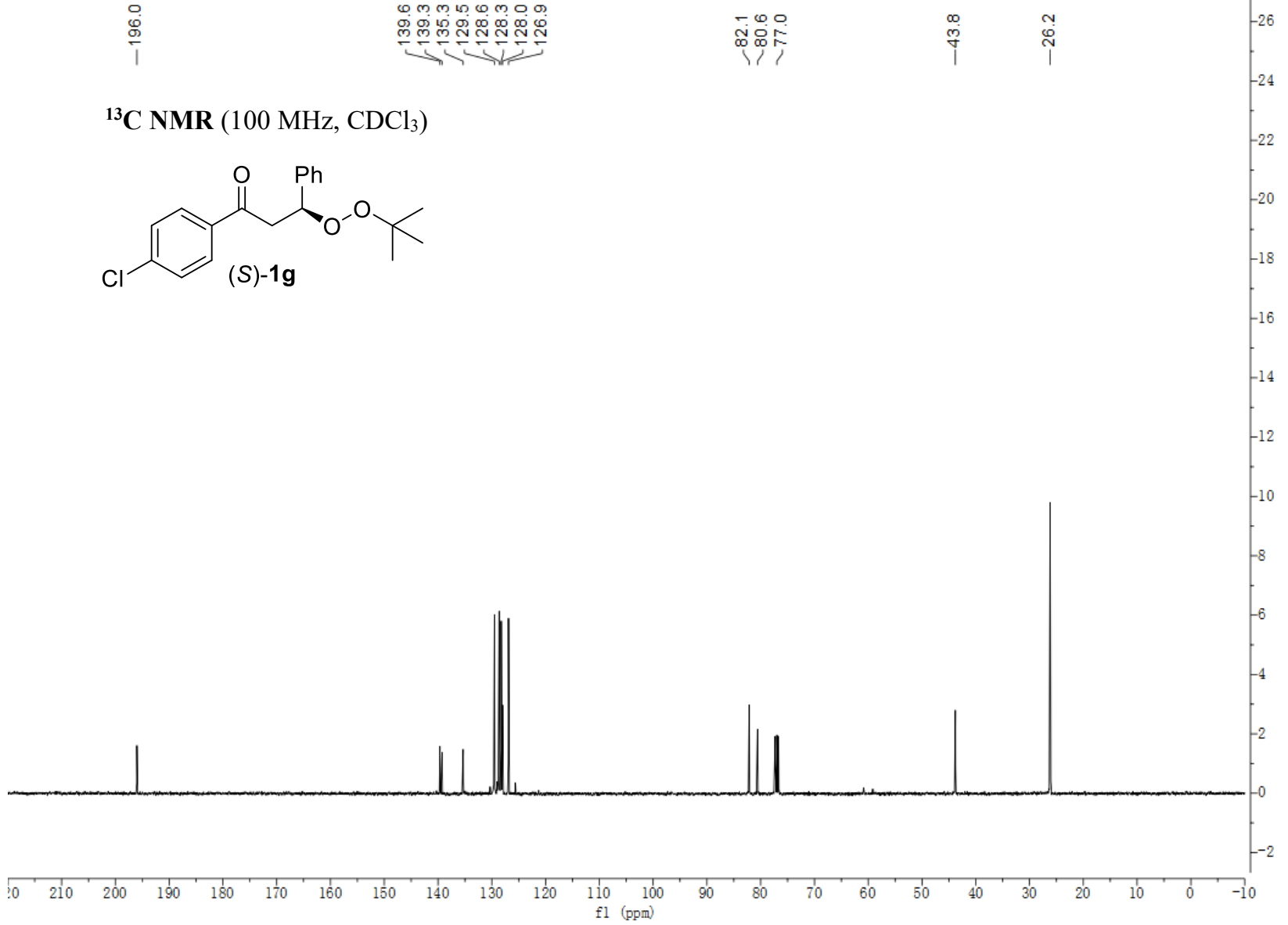


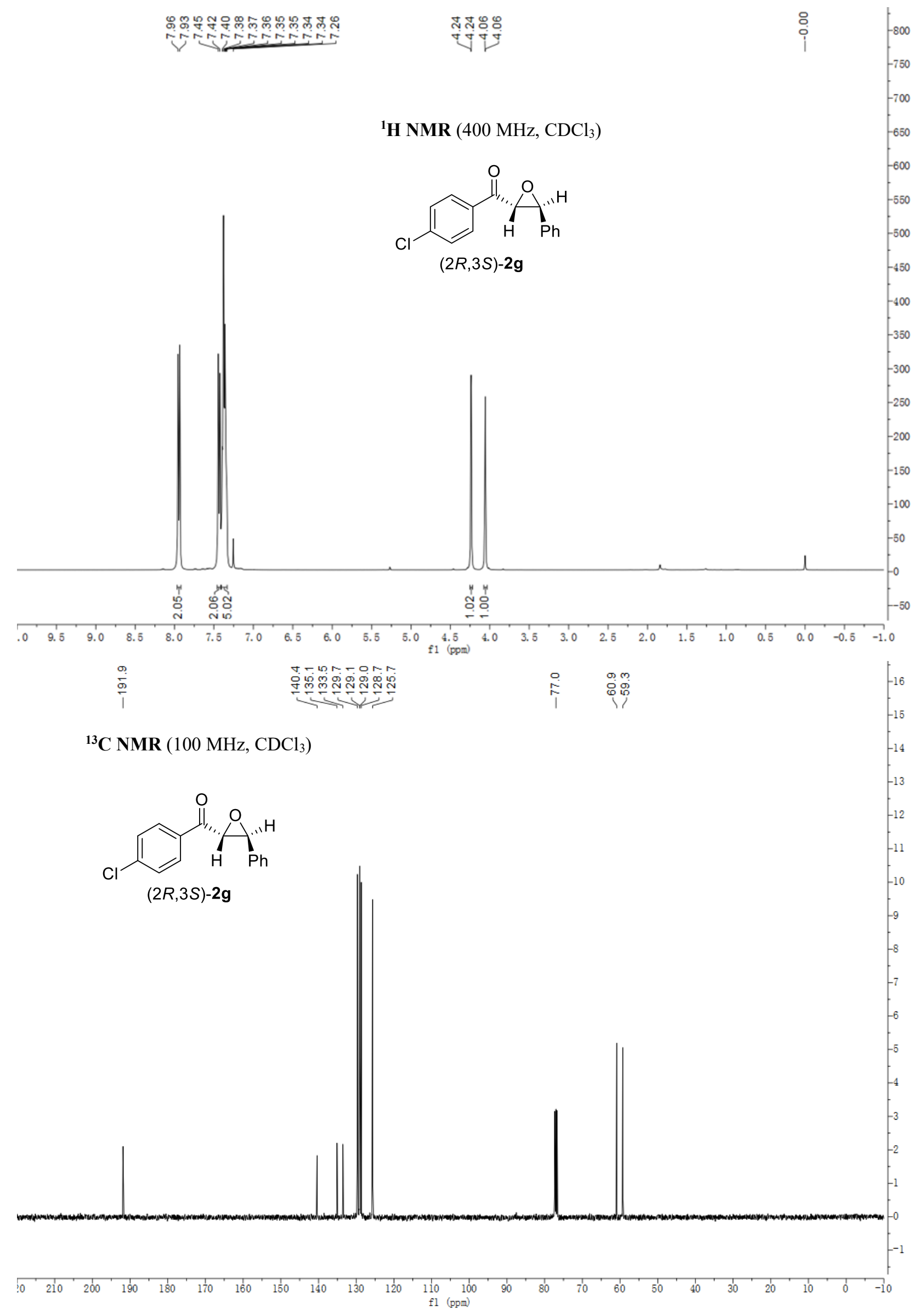




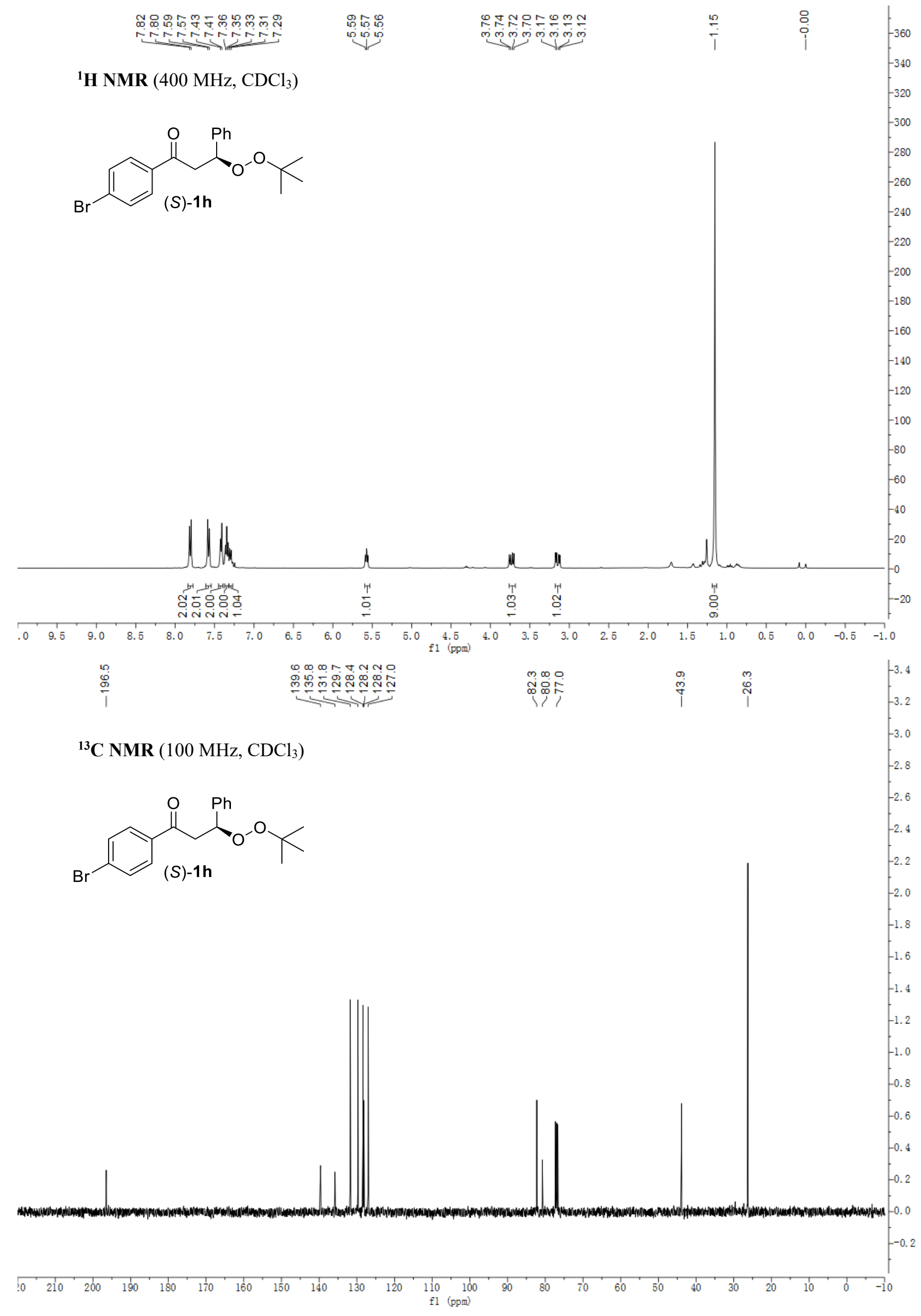


œ

rinirisiris

ֻֻู

$\stackrel{\circ}{\stackrel{2}{1}}$

${ }^{1} \mathbf{H}$ NMR $\left(400 \mathrm{MHz}, \mathrm{CDCl}_{3}\right)$<smiles>O=C(c1ccc(Br)cc1)[C@H](OC(c1ccccc1)c1ccccc1)C(c1ccccc1)c1ccccc1</smiles>

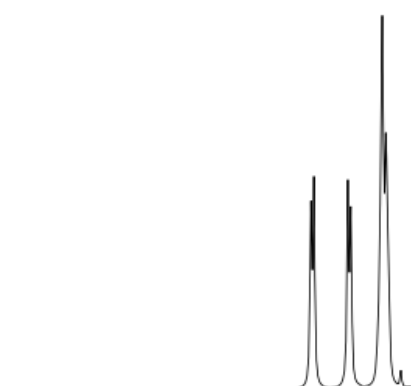

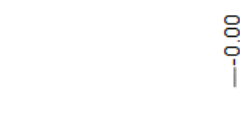

\begin{tabular}{l|l}
8 & -190 \\
$i$ & -180
\end{tabular}

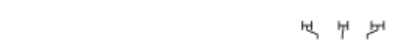

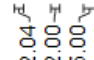

th

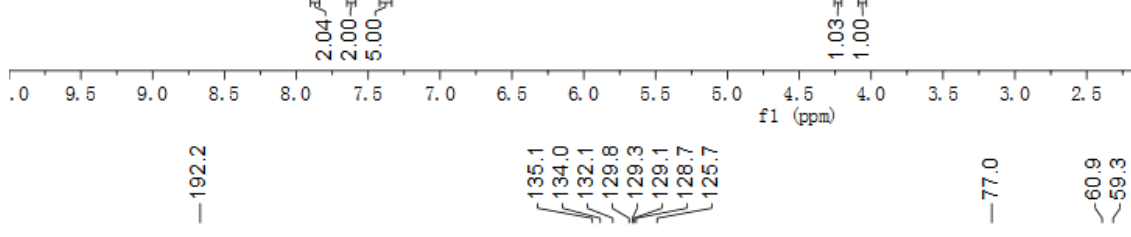

${ }^{13} \mathbf{C}$ NMR $\left(100 \mathrm{MHz}, \mathrm{CDCl}_{3}\right)$

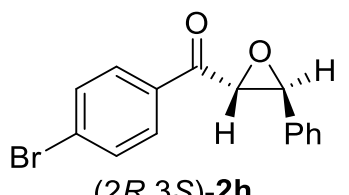

$(2 R, 3 S)-2 \mathbf{h}$

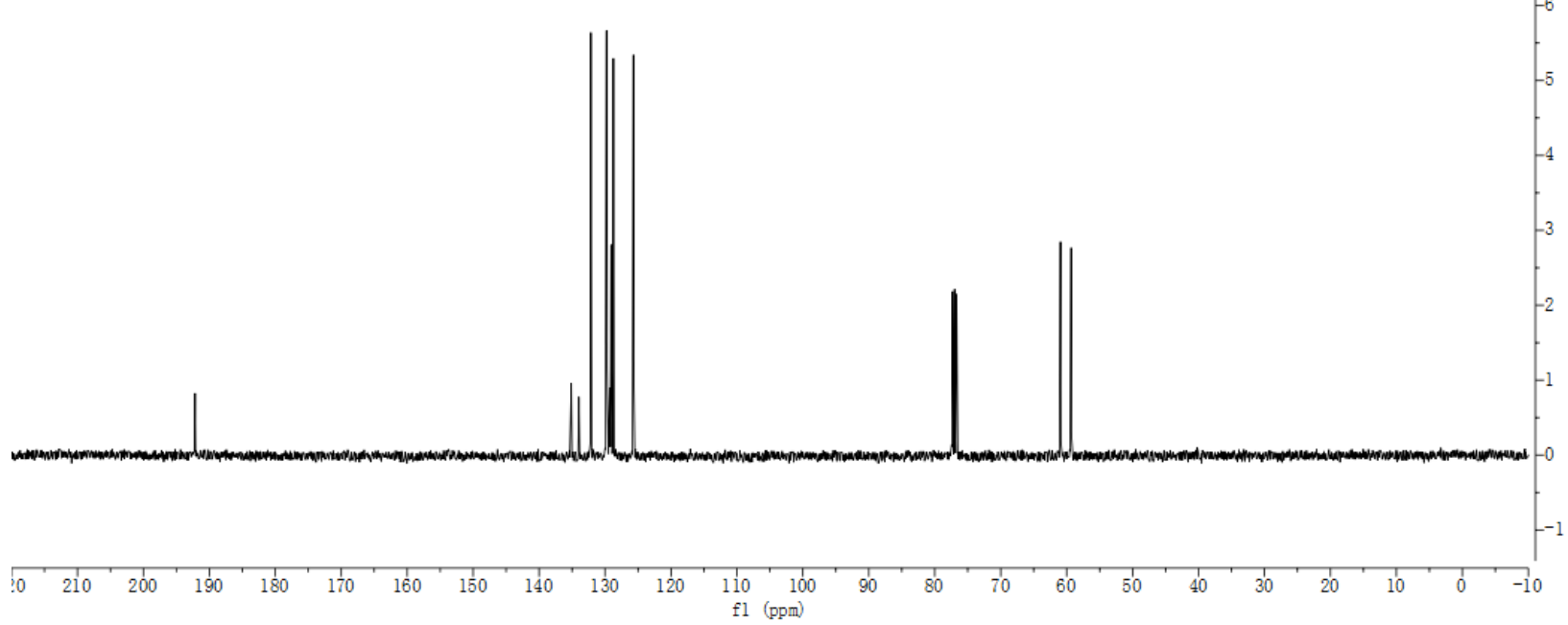


${ }^{1} \mathbf{H}$ NMR $\left(400 \mathrm{MHz}, \mathrm{CDCl}_{3}\right)$<smiles>CC(C)(C)OOC(CC(=O)c1ccc2ccccc2c1)c1ccccc1</smiles>

$(S)-1 \mathbf{i}$

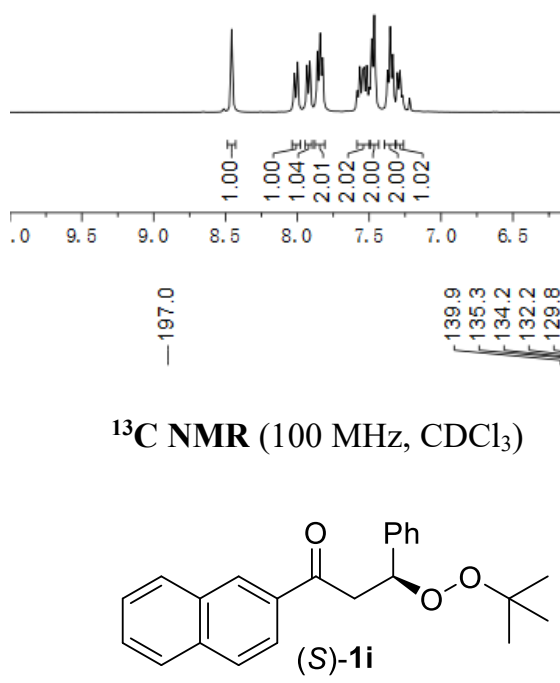

\section{r.}

mand
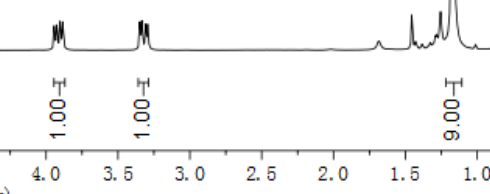

กำ

Nom

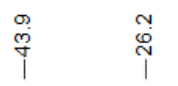

o

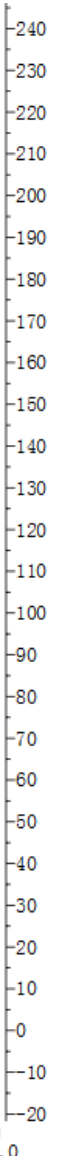

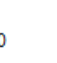




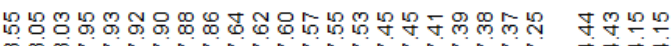

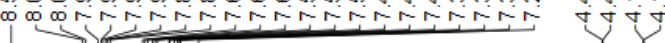

${ }^{1} \mathbf{H}$ NMR (400 MHz, $\left.\mathrm{CDCl}_{3}\right)$<smiles>O=C(c1ccc2ccccc2c1)[C@H]1O[C@H]1Pc1ccccc1</smiles>

$(2 R, 3 S)-\mathbf{i}$

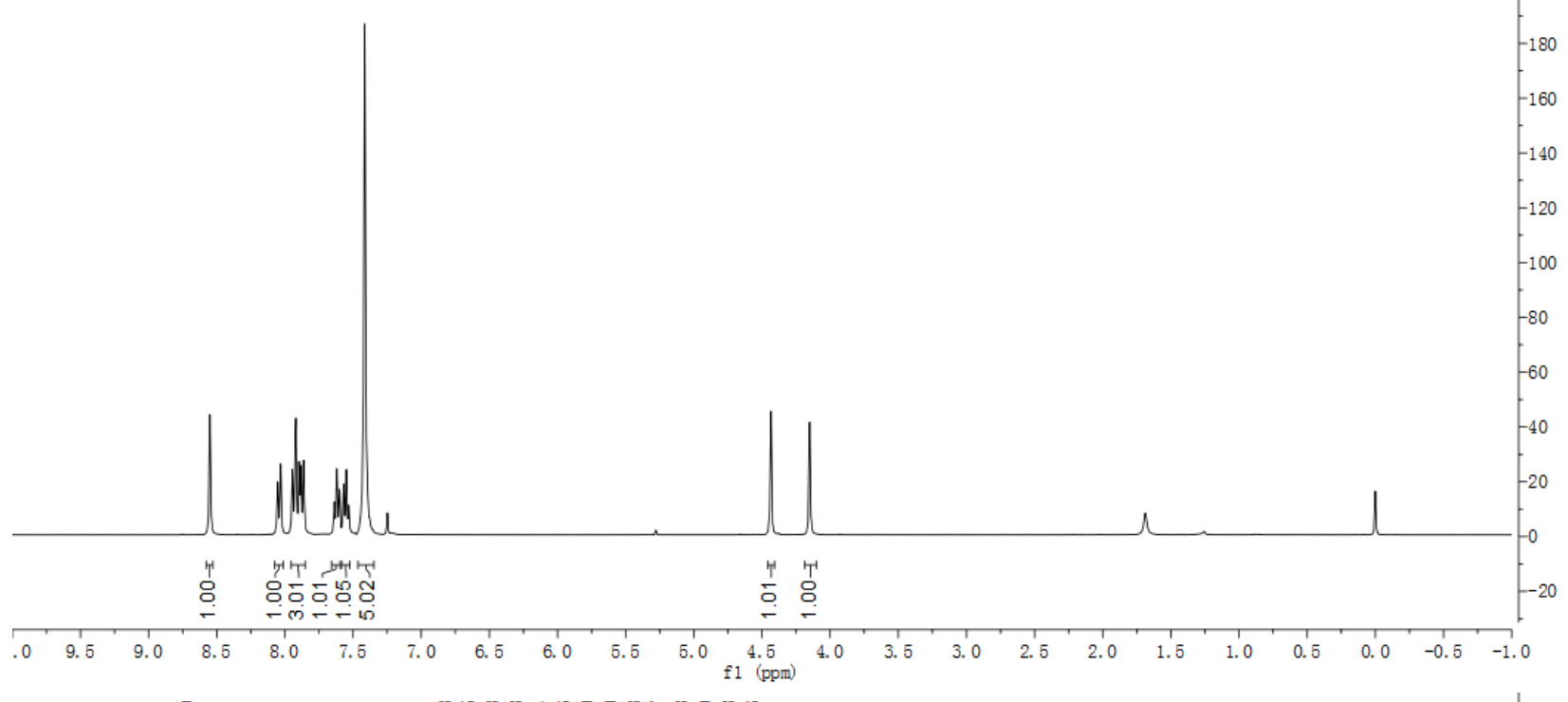
焉

${ }^{13}$ C NMR (100 MHz, $\left.\mathrm{CDCl}_{3}\right)$<smiles>O=C(c1ccc2ccccc2c1)[C@H]1O[C@H]1Pc1ccccc1</smiles>

$(2 R, 3 S)-\mathbf{2} \mathbf{i}$

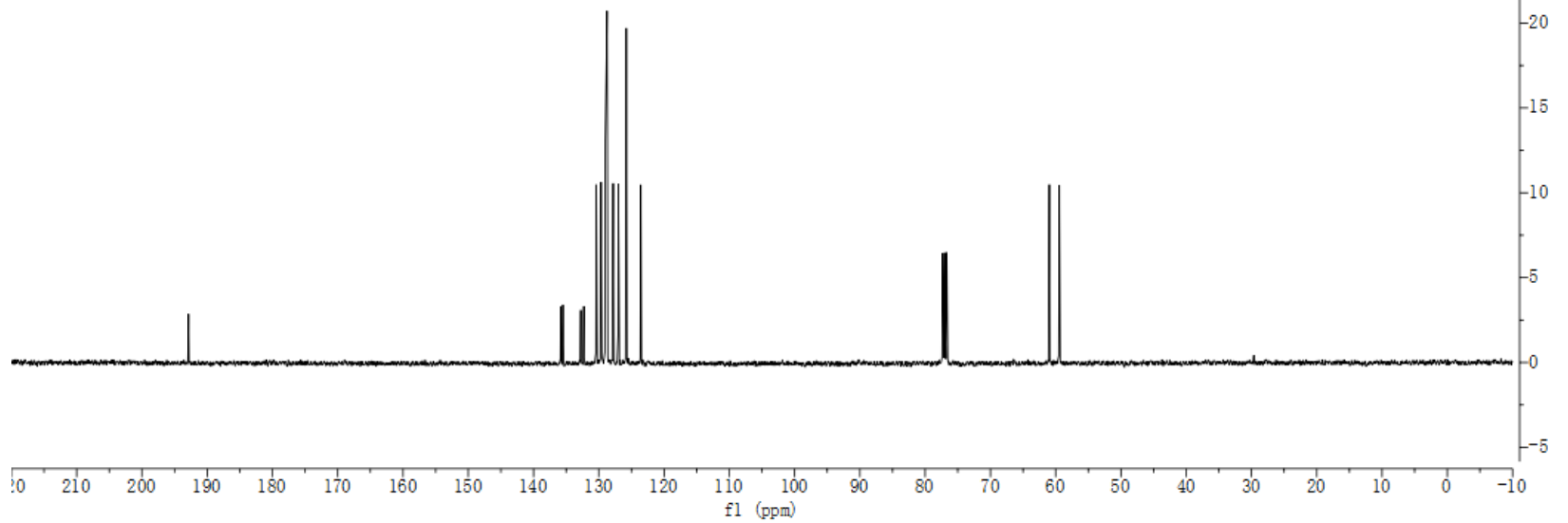


${ }^{1} \mathbf{H}$ NMR $\left(400 \mathrm{MHz}, \mathrm{CDCl}_{3}\right)$<smiles>CC(C)(C)OOC(CC(=O)c1ccco1)c1ccccc1</smiles>

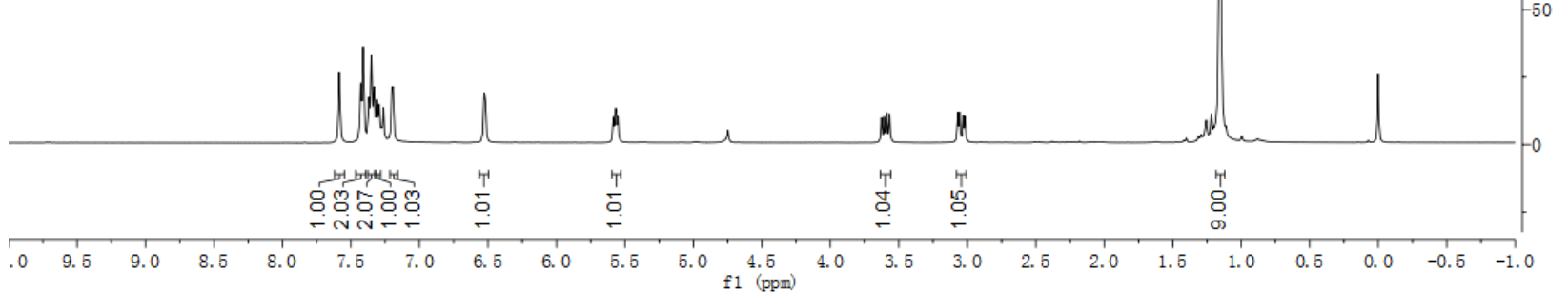

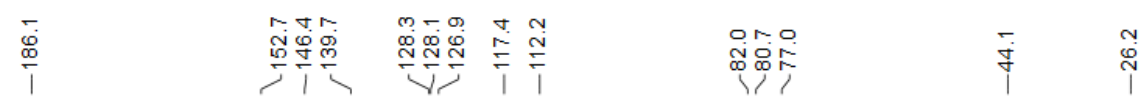

${ }^{13} \mathbf{C}$ NMR $\left(100 \mathrm{MHz}, \mathrm{CDCl}_{3}\right)$<smiles>CC(C)(C)OOC(CC(=O)c1ccco1)c1ccccc1</smiles>

(S)-1j

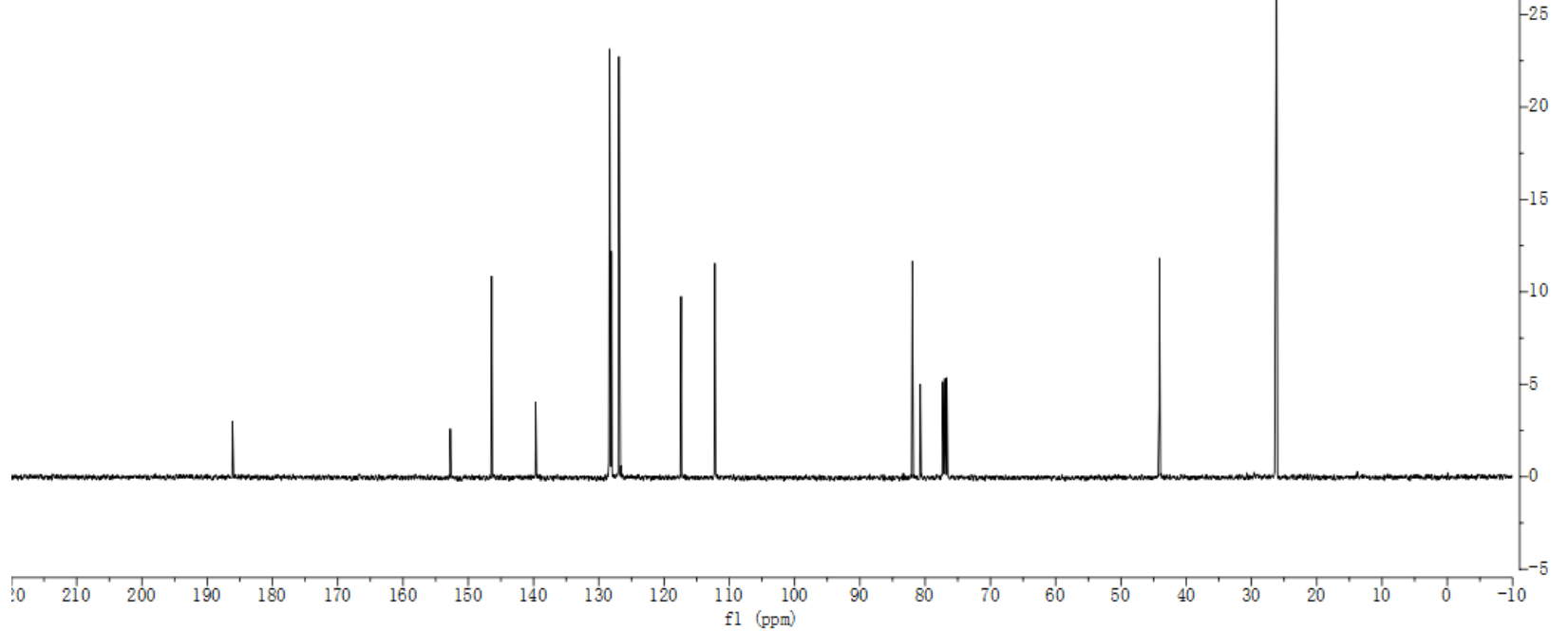




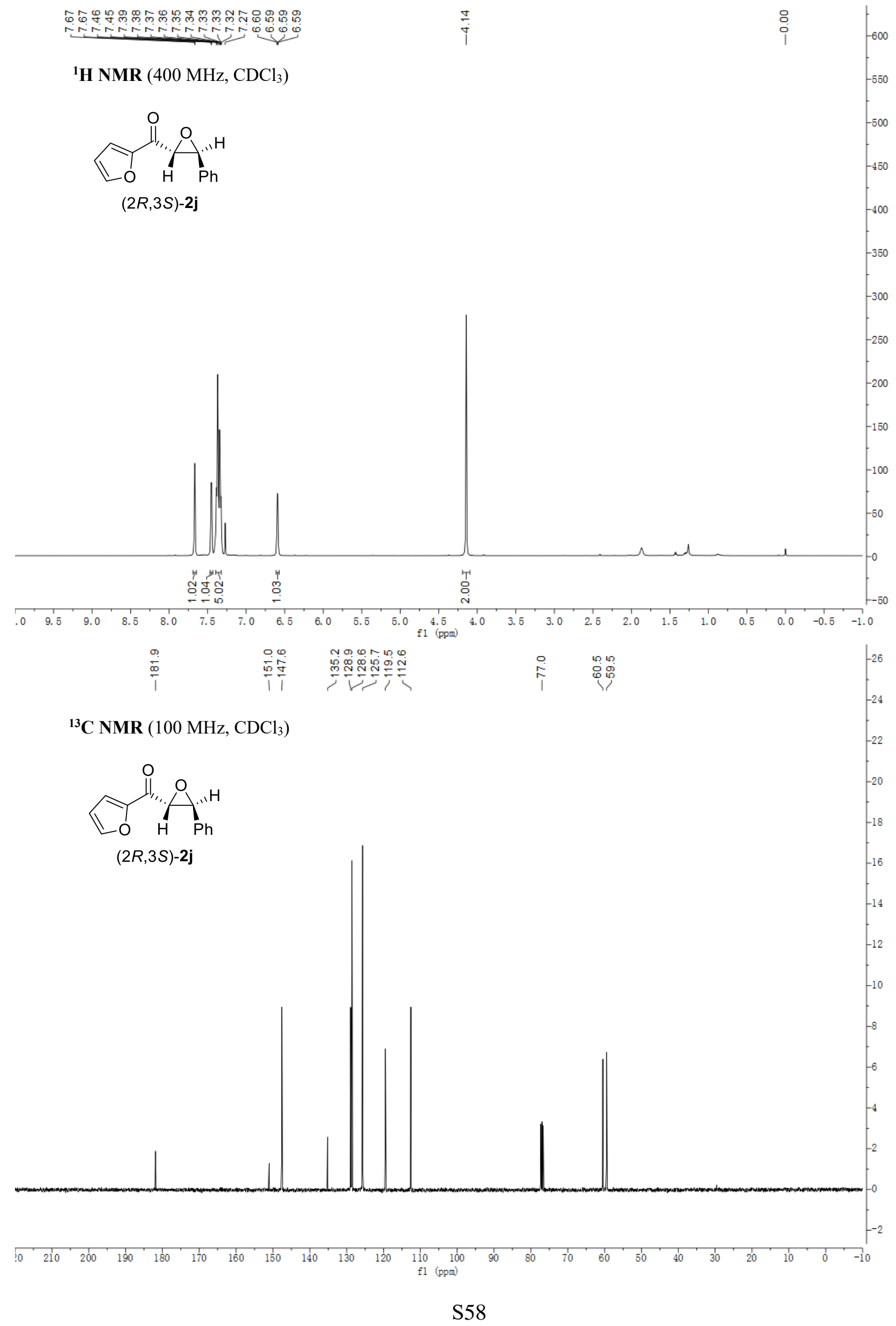


${ }^{1} \mathbf{H}$ NMR $\left(400 \mathrm{MHz}, \mathrm{CDCl}_{3}\right)$<smiles>CC(C)(C)OOC(CC(=O)c1cccs1)c1ccccc1</smiles>

(S)-1k
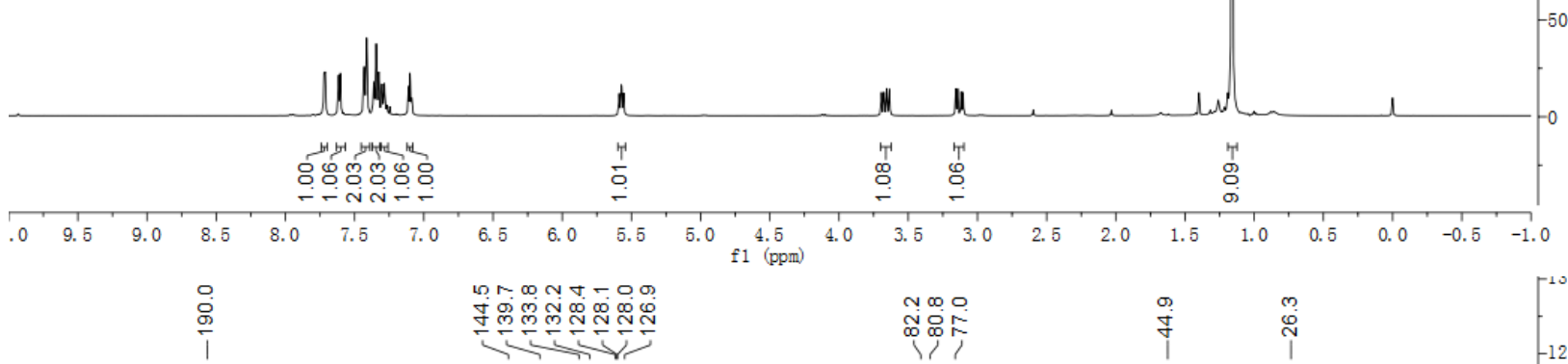

$\underset{\infty}{\operatorname{Non}} \stackrel{\infty}{\infty}$

Nof

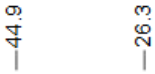

${ }^{13} \mathbf{C}$ NMR $\left(100 \mathrm{MHz}, \mathrm{CDCl}_{3}\right)$<smiles>CC(C)(C)OOC(CC(=O)c1cccs1)c1ccccc1</smiles>

(S)-1k

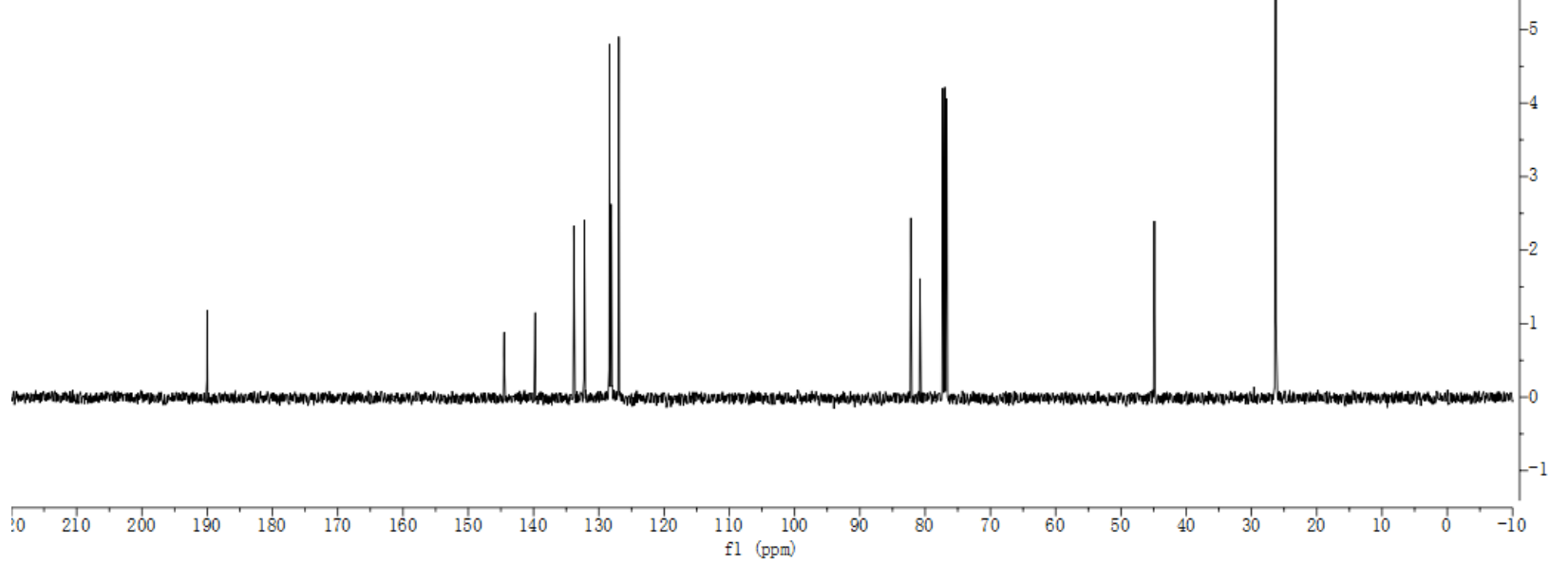


${ }^{1} \mathbf{H}$ NMR (400 MHz, $\left.\mathrm{CDCl}_{3}\right)$

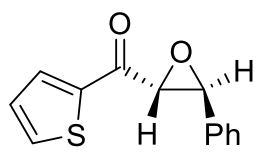

$(2 R, 3 S)-\mathbf{2 k}$

${ }^{13} \mathbf{C}$ NMR $\left(100 \mathrm{MHz}, \mathrm{CDCl}_{3}\right)$<smiles>O=C(c1cccs1)[C@H]1O[C@H]1c1ccccc1</smiles>

$(2 R, 3 S)-\mathbf{2 k}$

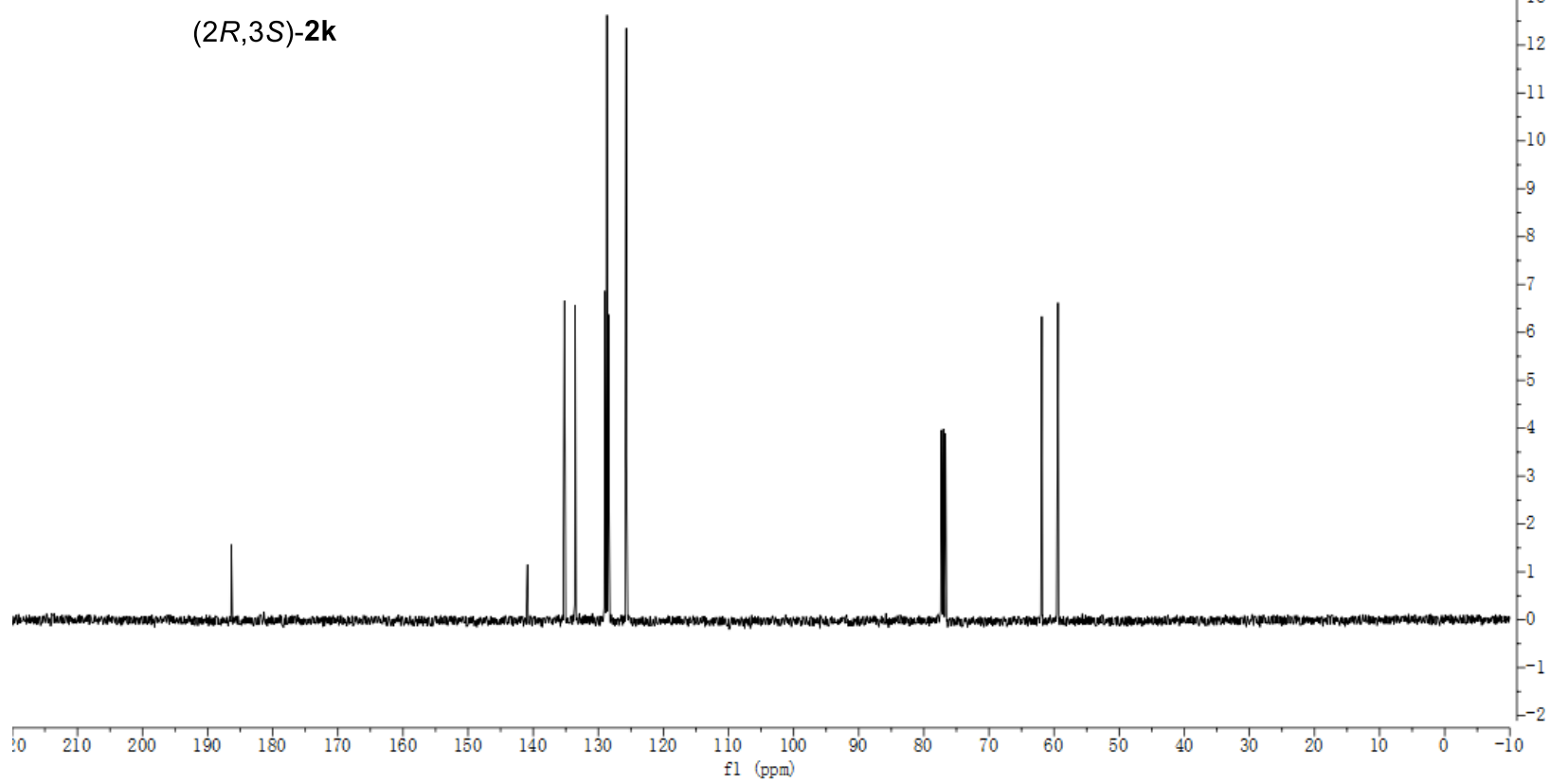


${ }^{1} \mathbf{H}$ NMR $\left(400 \mathrm{MHz}, \mathrm{CDCl}_{3}\right)$<smiles>Cc1ccc(C(CC(=O)c2ccccc2)OOC(C)(C)C)cc1</smiles>

(S)-1I



${ }^{13}$ C NMR $\left(100 \mathrm{MHz}, \mathrm{CDCl}_{3}\right)$<smiles>Cc1ccc(C(CC(=O)c2ccccc2)OOC(C)(C)C)cc1</smiles>

(S)-1I

\section{}

C NMR (100 $\left.\mathrm{MH}_{2}, \mathrm{CDCl}_{3}\right)$

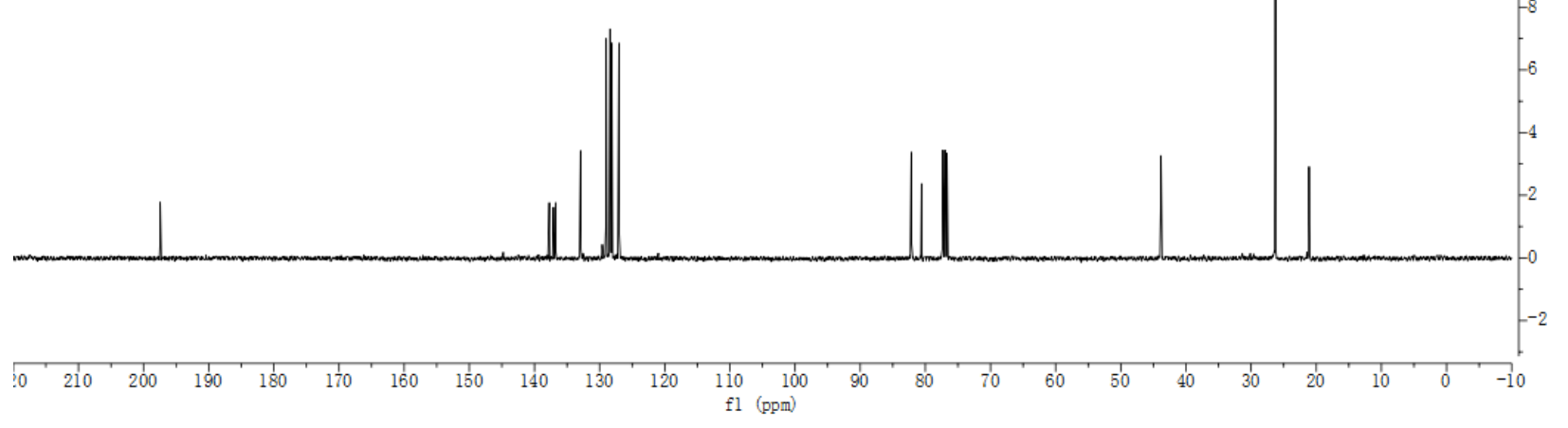




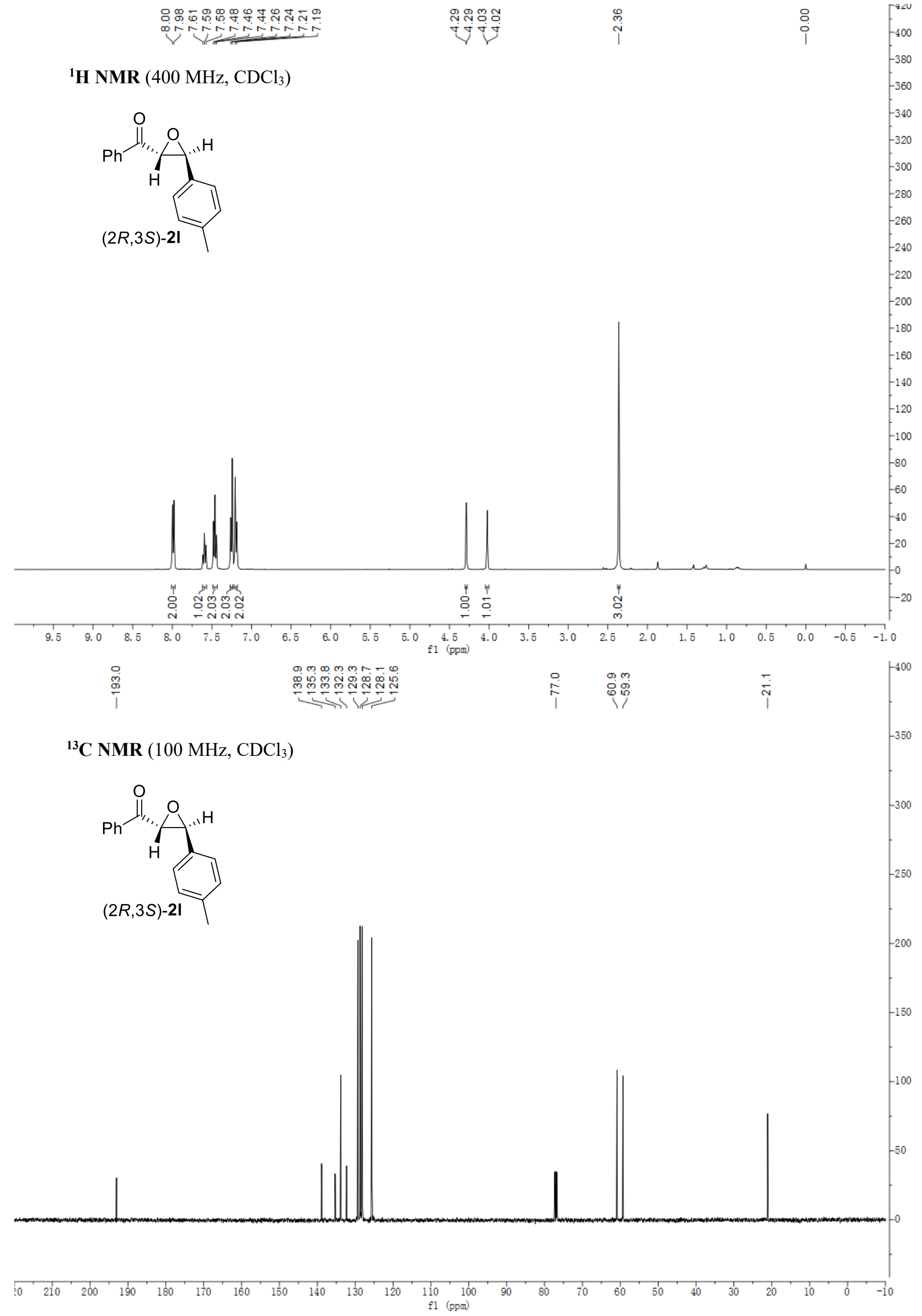


${ }^{1} \mathbf{H}$ NMR $\left(400 \mathrm{MHz}, \mathrm{CDCl}_{3}\right)$<smiles>CC(C)(C)OOC(CC(=O)c1ccccc1)c1ccc(C(C)(C)C)cc1</smiles>

(S)-1m

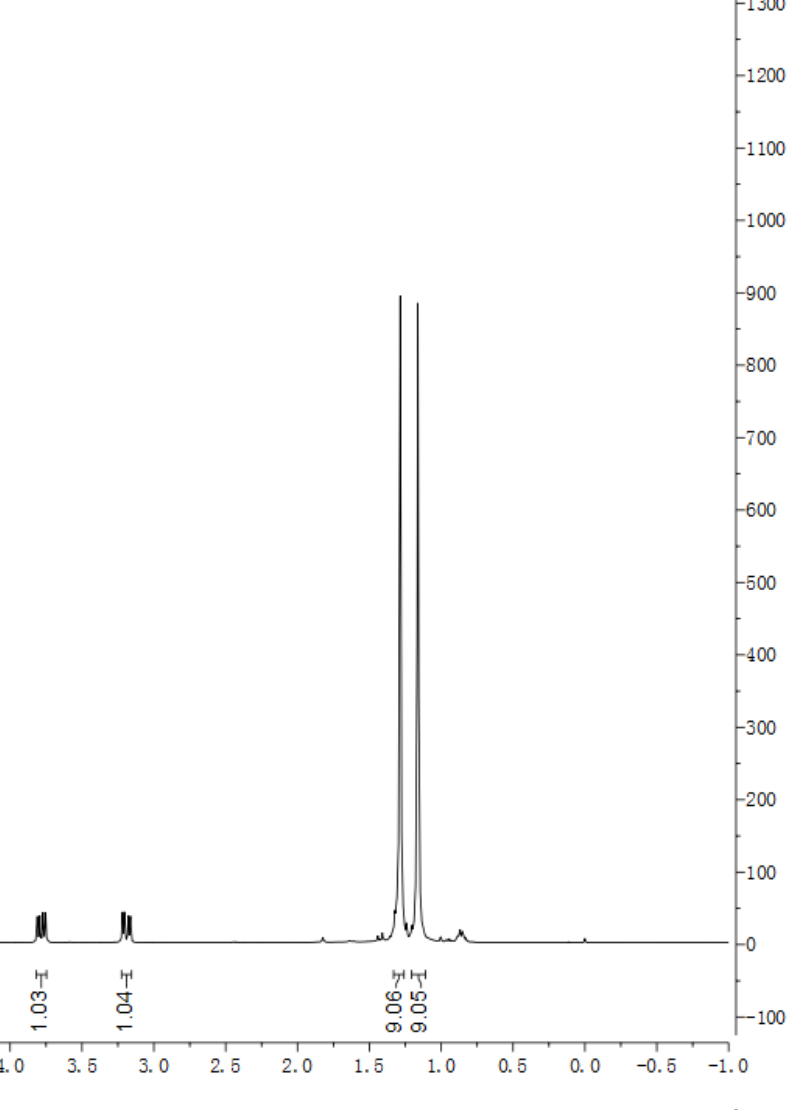

管

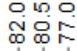

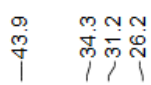

${ }^{13} \mathbf{C}$ NMR $\left(100 \mathrm{MHz}, \mathrm{CDCl}_{3}\right)$<smiles>CC(C)(C)OOC(CC(=O)c1ccccc1)c1ccc(C(C)(C)C)cc1</smiles>

(S)-1m

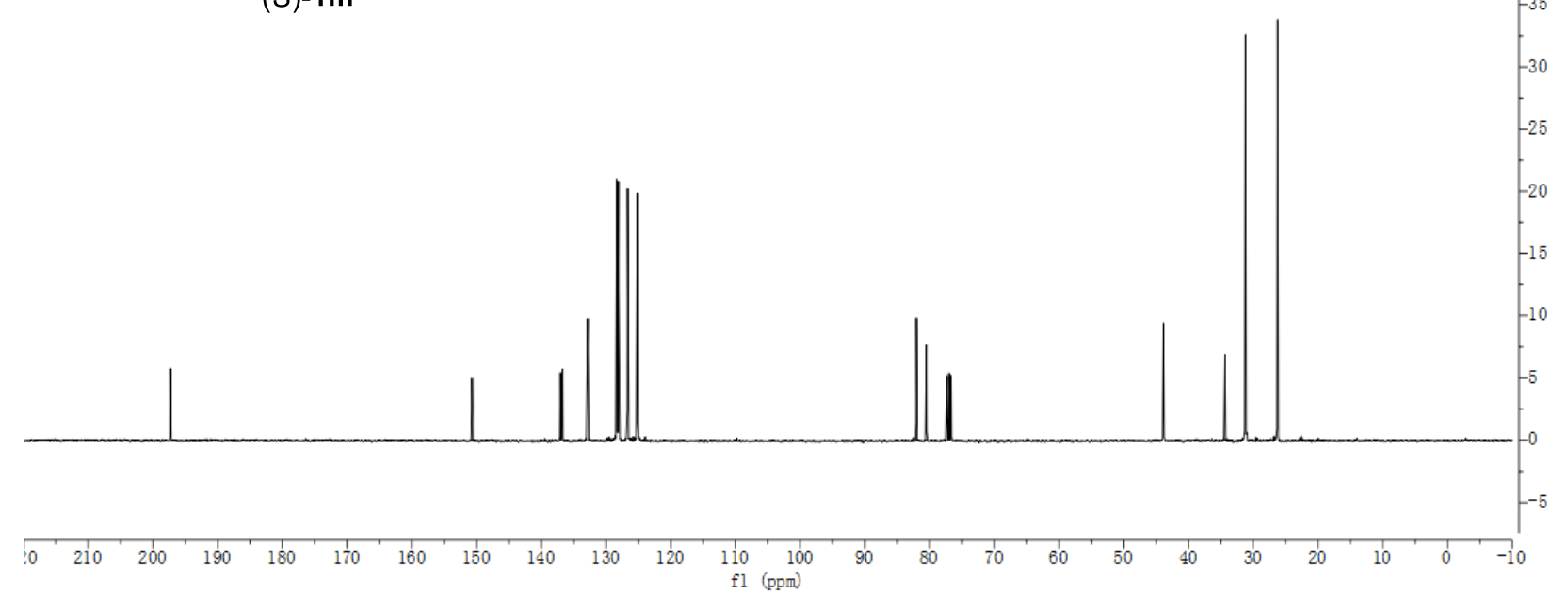




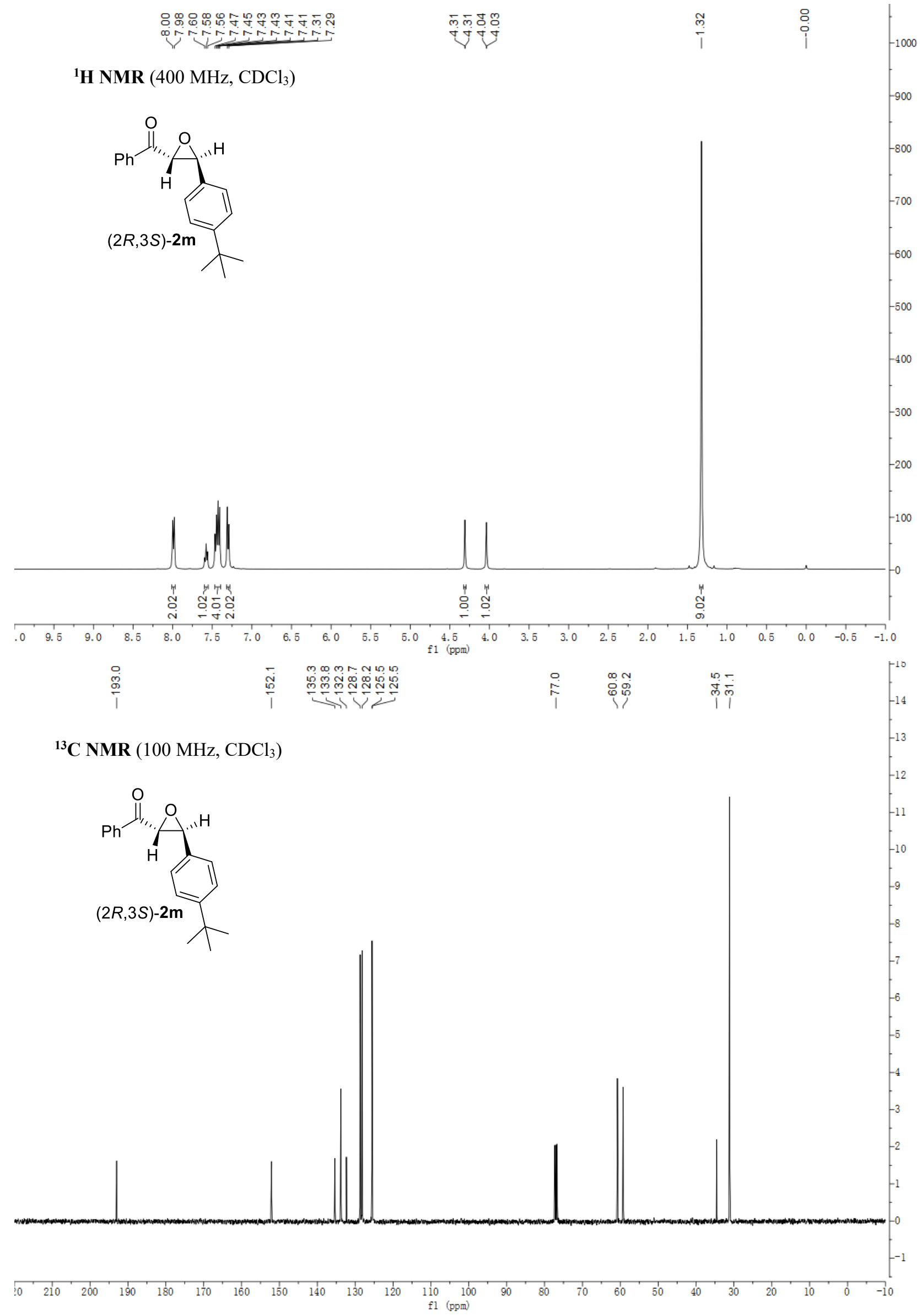




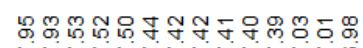
무용

10

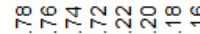

ले लेल ले

$\rightarrow$ iूल

${ }^{1} \mathbf{H}$ NMR $\left(400 \mathrm{MHz}, \mathrm{CDCl}_{3}\right)$<smiles>CC(C)(C)OOC(CC(=O)c1ccccc1)c1ccc(F)cc1</smiles>

(S)-1n

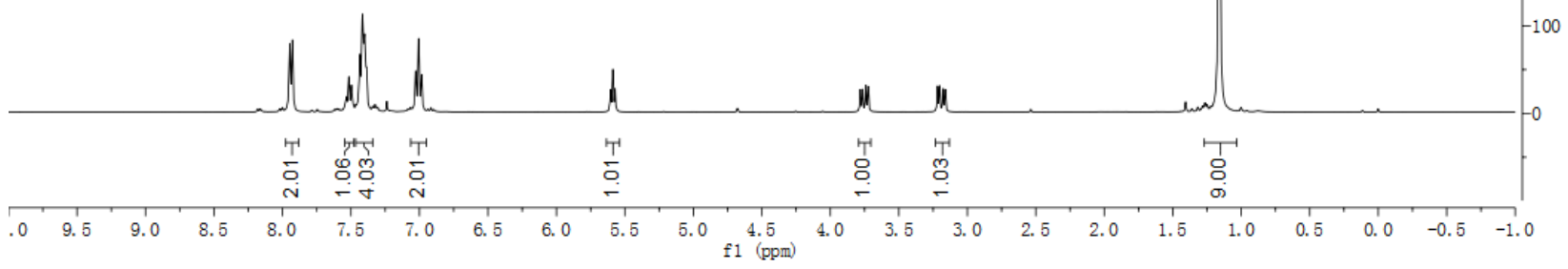

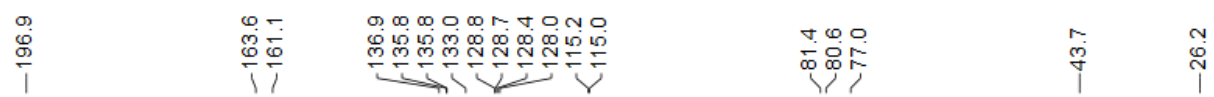

${ }^{13} \mathbf{C}$ NMR $\left(100 \mathrm{MHz}, \mathrm{CDCl}_{3}\right)$<smiles>CC(C)(C)OOC(CC(=O)c1ccccc1)c1ccc(F)cc1</smiles>

(S)-1n

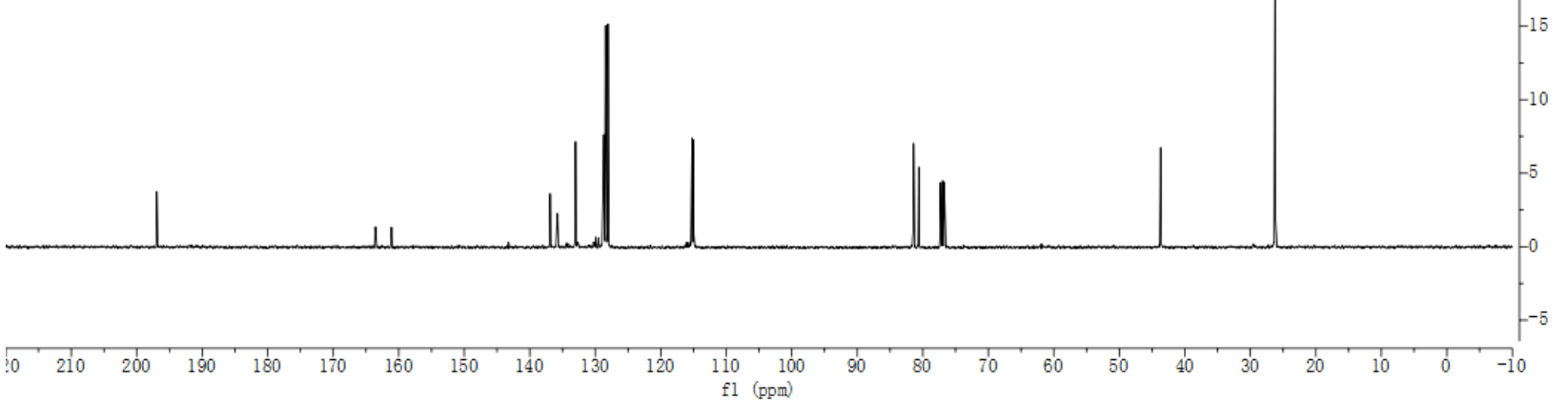




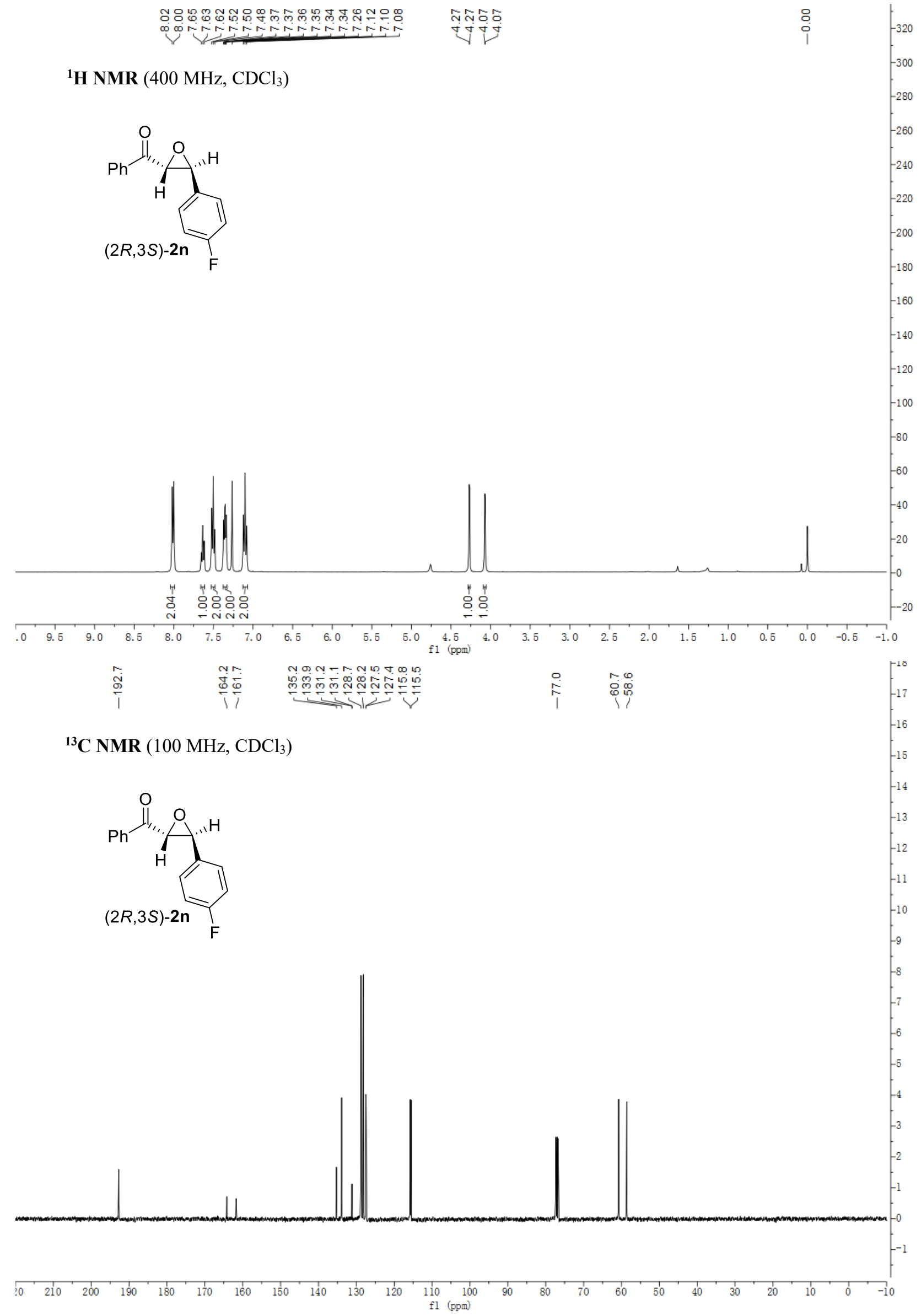


${ }^{1} \mathbf{H}$ NMR $\left(400 \mathrm{MHz}, \mathrm{CDCl}_{3}\right)$<smiles>CC(C)(C)OOC(CC(=O)c1ccccc1)c1ccc(Cl)cc1</smiles>

(S)-10

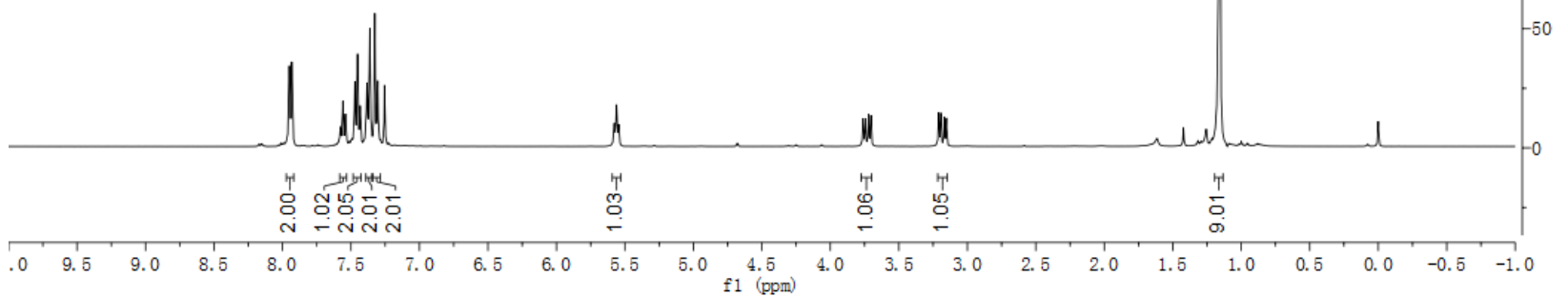

${ }^{13} \mathbf{C}$ NMR $\left(100 \mathrm{MHz}, \mathrm{CDCl}_{3}\right)$<smiles>CC(C)(C)OOC(CC(=O)c1ccccc1)c1ccc(Cl)cc1</smiles>

(S)-10

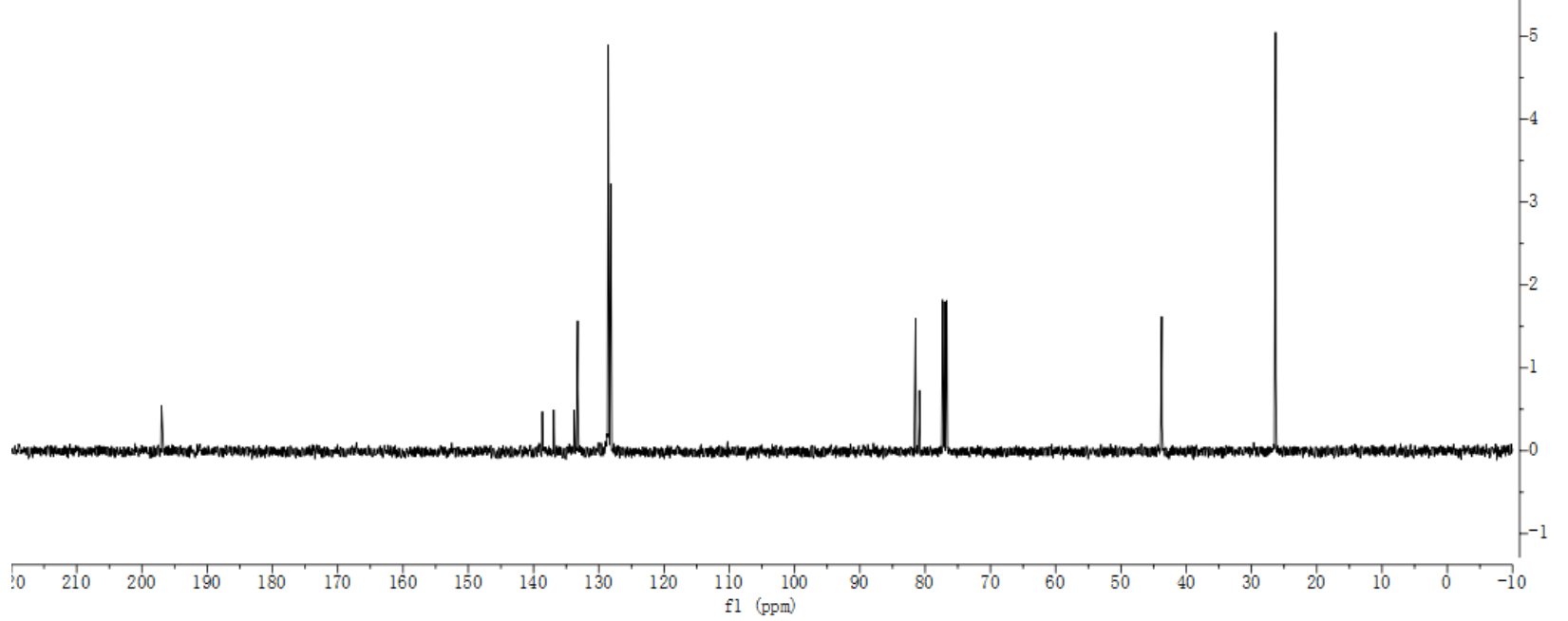




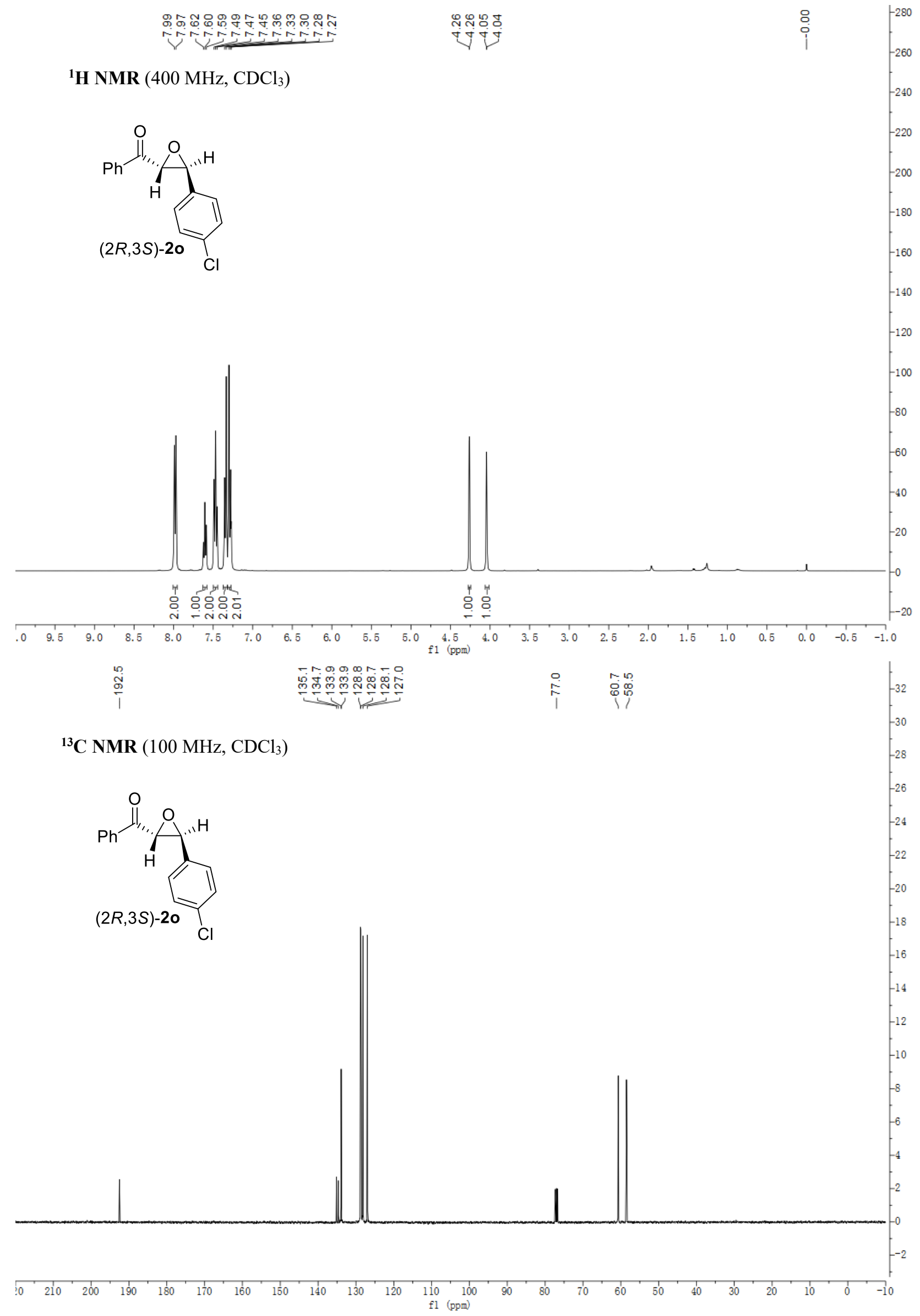


ณ

rint

V

${ }^{1} \mathbf{H}$ NMR (400 MHz, $\mathrm{CDCl}_{3}$ )<smiles>CC(C)(C)OOC(CC(=O)c1ccccc1)c1ccc(Br)cc1</smiles>

(S)-1p

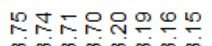

लं लंखुल लुल

$$
\stackrel{\leftrightarrow}{i} \stackrel{0}{i}
$$

$\stackrel{8}{i}$

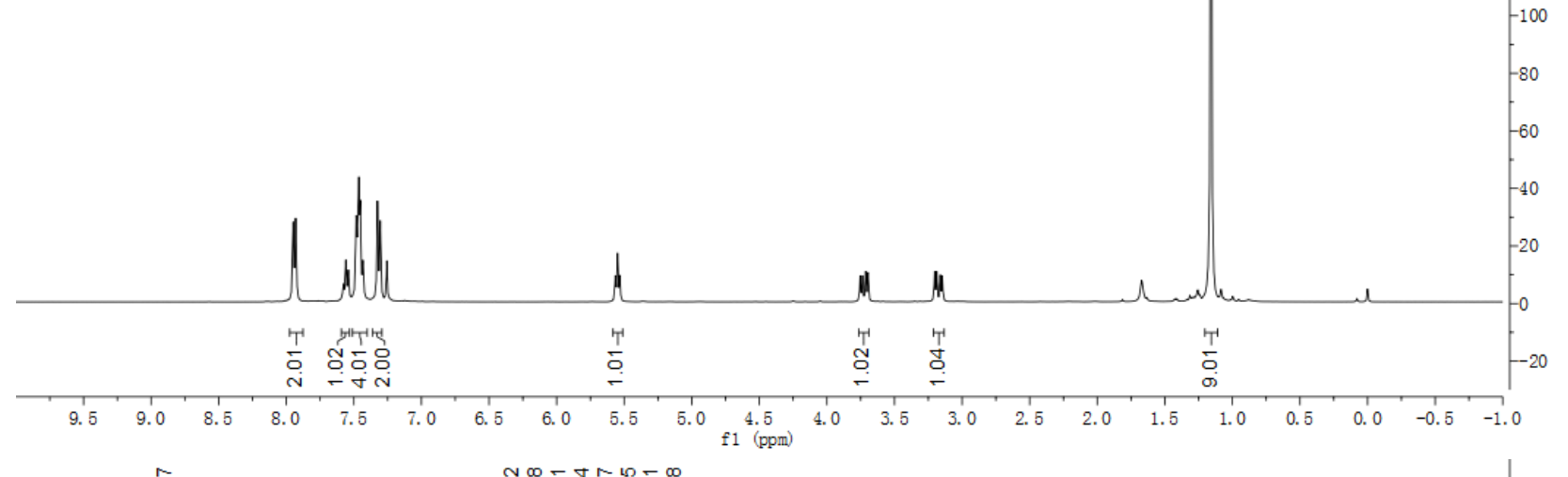

$\frac{0}{1}$

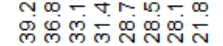

$\underbrace{-5}=$

뭉요

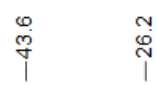

${ }^{13}$ C NMR (100 MHz, $\left.\mathrm{CDCl}_{3}\right)$<smiles>CC(C)(C)OOC(CC(=O)c1ccccc1)c1ccc(Br)cc1</smiles>

(S)-1p

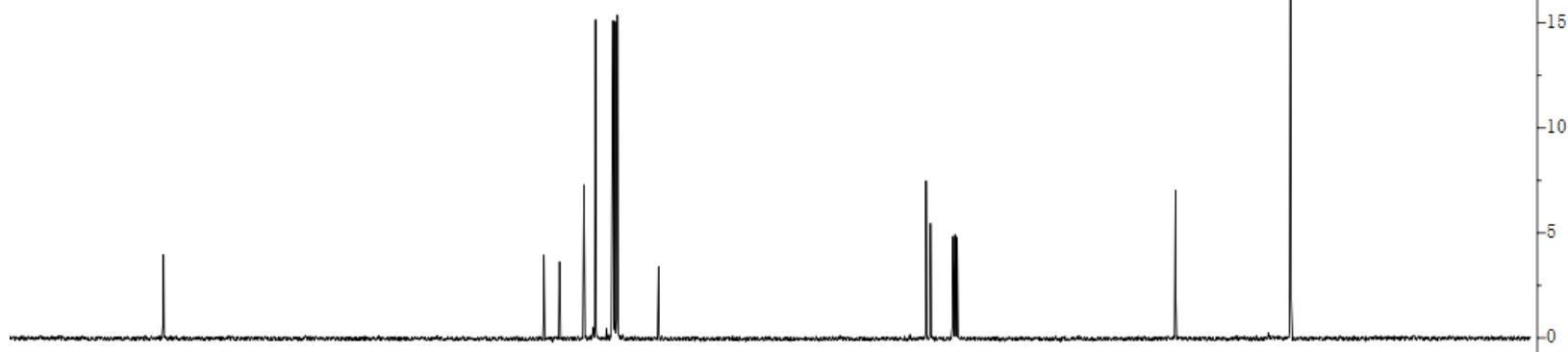

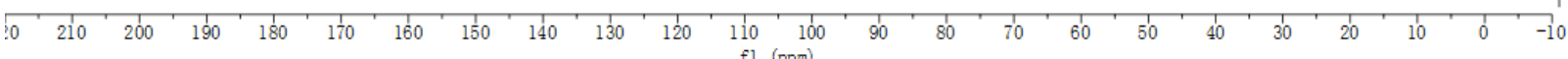




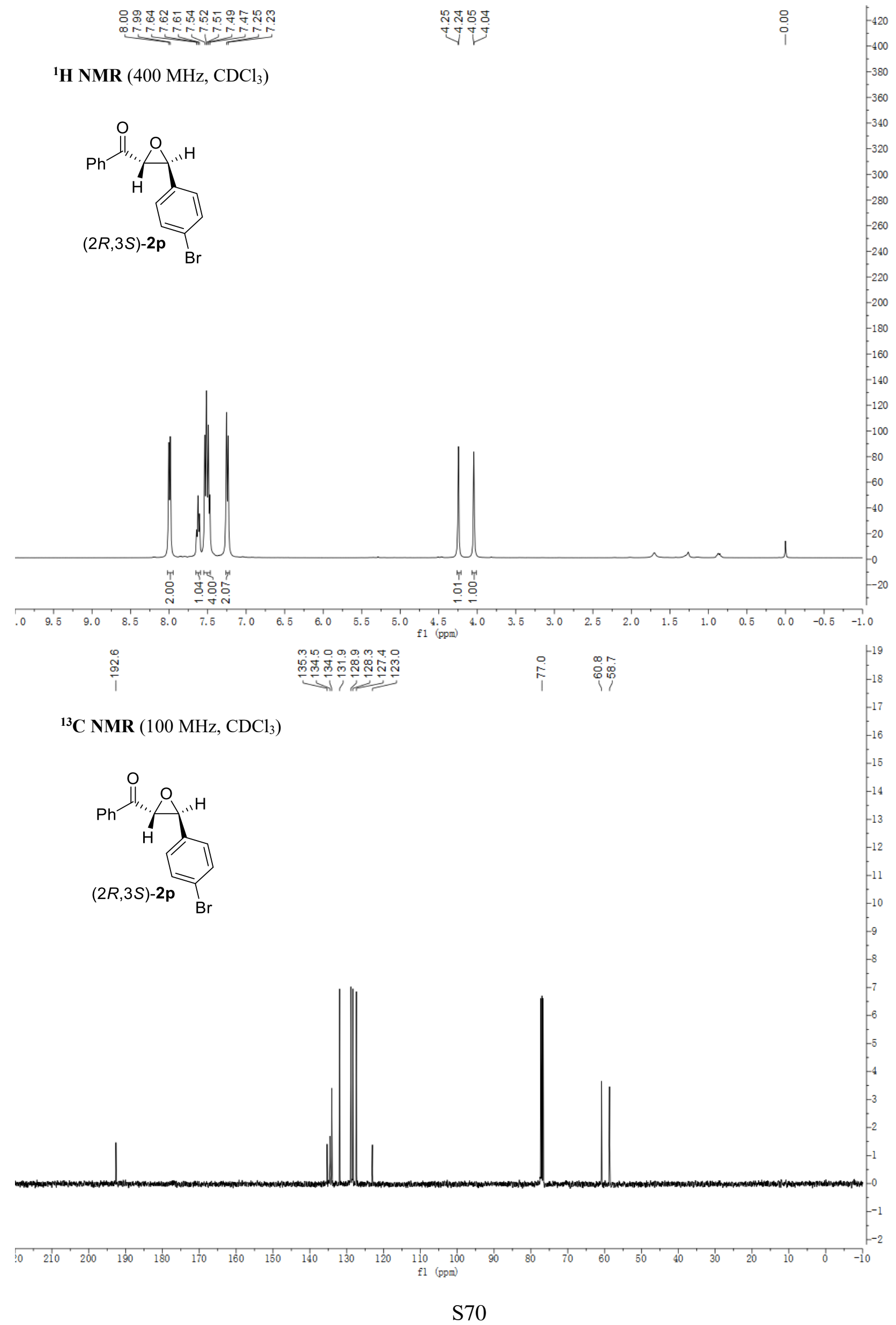


${ }^{1} \mathbf{H}$ NMR $\left(400 \mathrm{MHz}, \mathrm{CDCl}_{3}\right)$

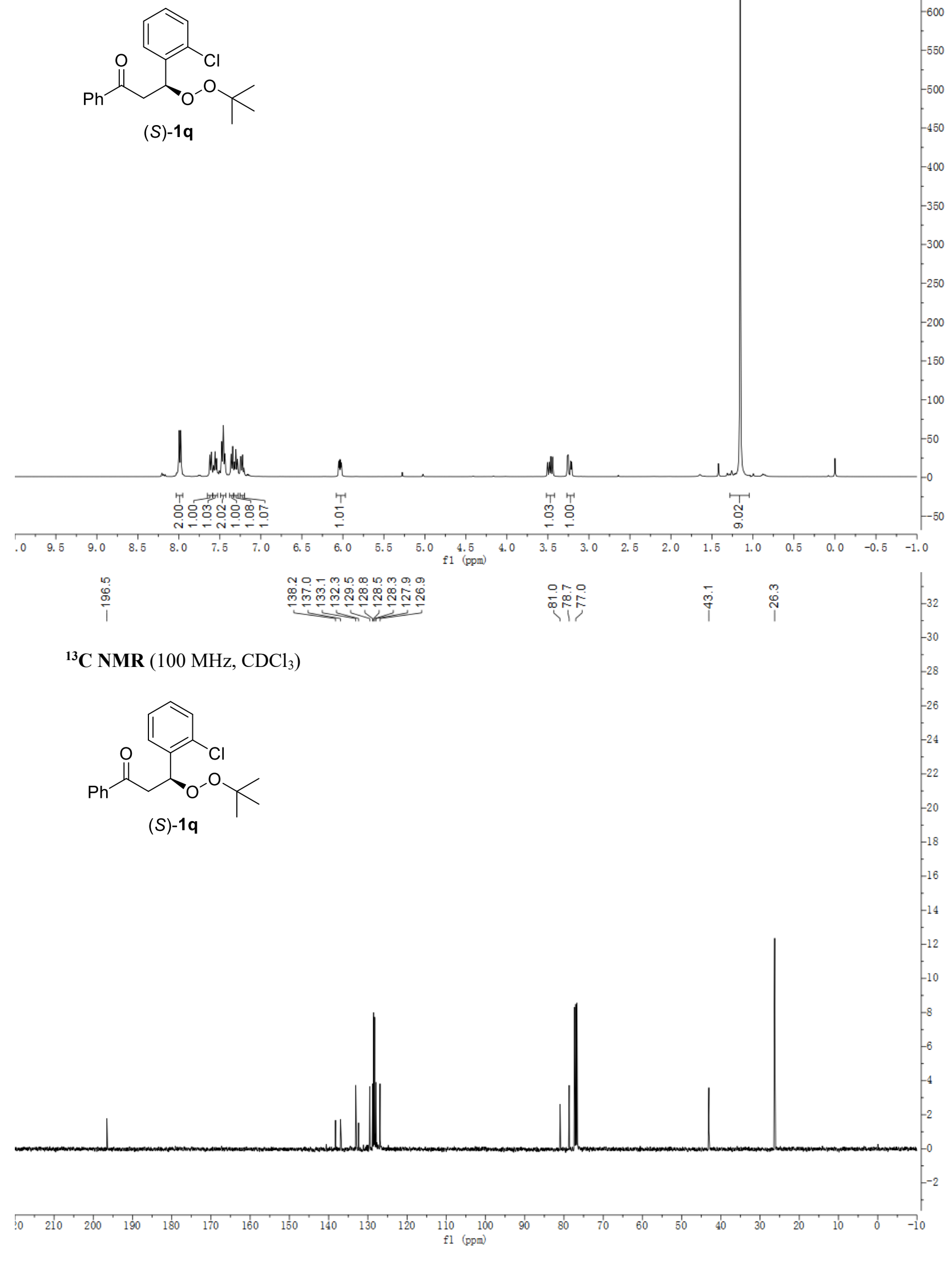

(S)-1q

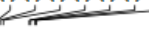


${ }^{\mathbf{1}} \mathbf{H}$ NMR $\left(400 \mathrm{MHz}, \mathrm{CDCl}_{3}\right)$

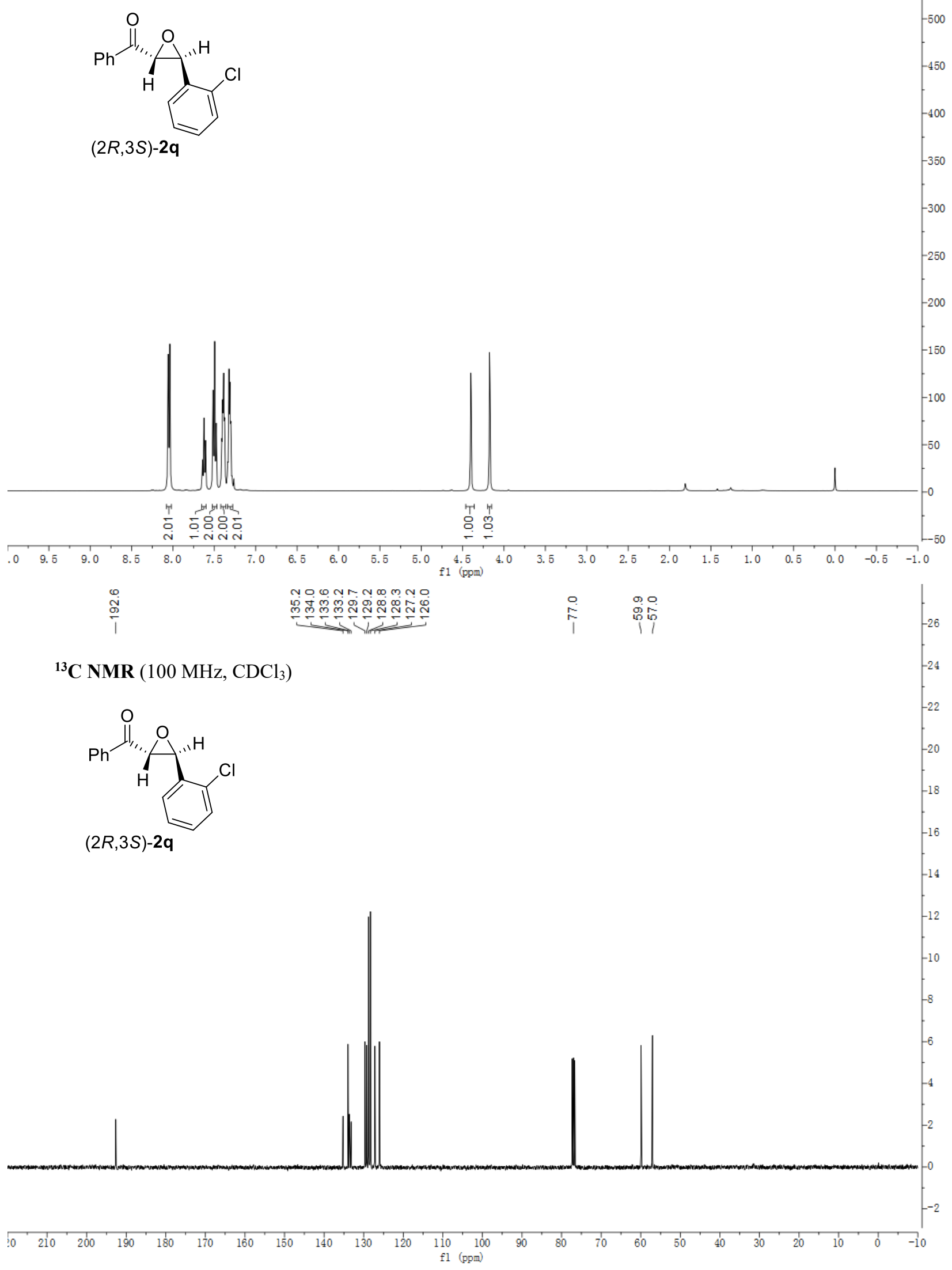


${ }^{1} \mathbf{H}$ NMR $\left(400 \mathrm{MHz}, \mathrm{CDCl}_{3}\right)$<smiles>CC(C)(C)OOC(CC(=O)c1ccccc1)c1cccc(Br)c1</smiles>

$(S)-1 r$

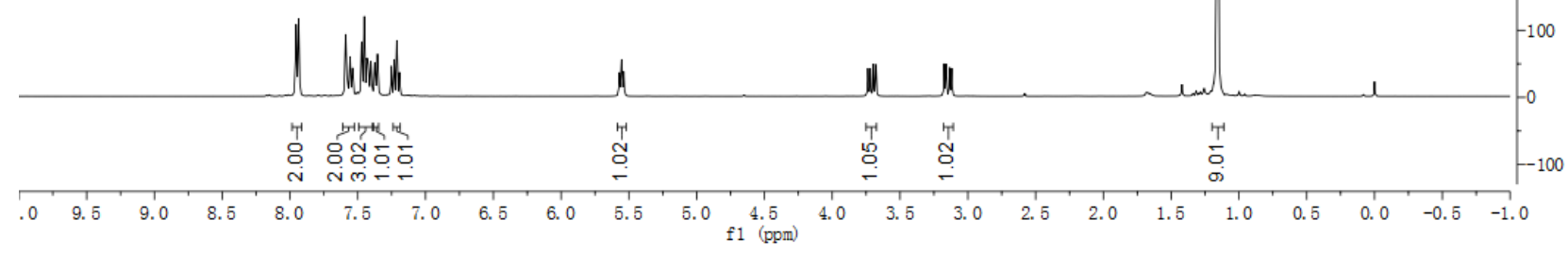
$\frac{\infty}{\infty}$

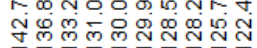
莳帝

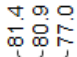

${ }^{13}$ C NMR $\left(100 \mathrm{MHz}, \mathrm{CDCl}_{3}\right)$<smiles>CC(C)(C)OOC(CC(=O)c1ccccc1)c1cccc(Br)c1</smiles>

$(S)-1 r$

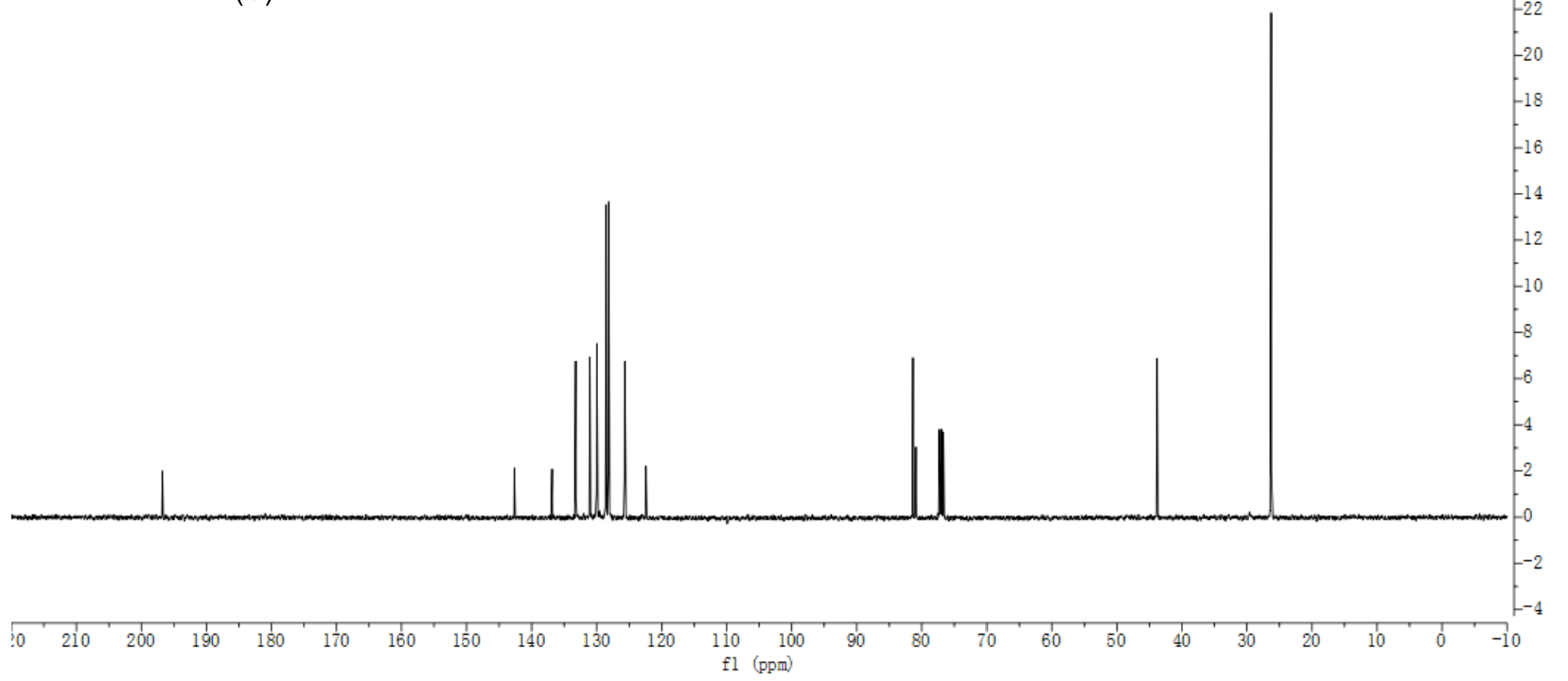




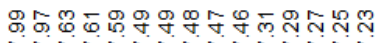

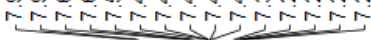

กับำกำ

サேं

${ }^{1} \mathbf{H}$ NMR $\left(400 \mathrm{MHz}, \mathrm{CDCl}_{3}\right)$<smiles></smiles>

$(2 R, 3 S)-2 \mathrm{r}$ $\mathrm{Br}$

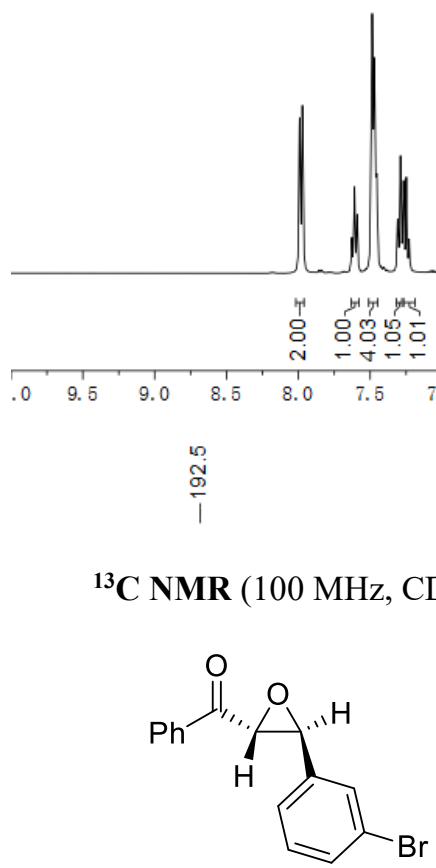

$(2 R, 3 S)-2 \mathbf{r}$

m.

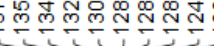

$\stackrel{0}{i} \quad \stackrel{9}{10}$

急

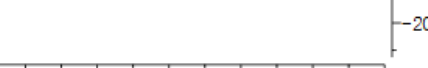
f1 (ppm)

\section{ip}

$\left.\mathrm{DCl}_{3}\right)$ 
${ }^{1} \mathbf{H}$ NMR $\left(400 \mathrm{MHz}, \mathrm{CDCl}_{3}\right)$<smiles>CC(C)(OOC(CC(=O)c1ccccc1)c1ccccc1)c1ccccc1</smiles>

(S)-1s

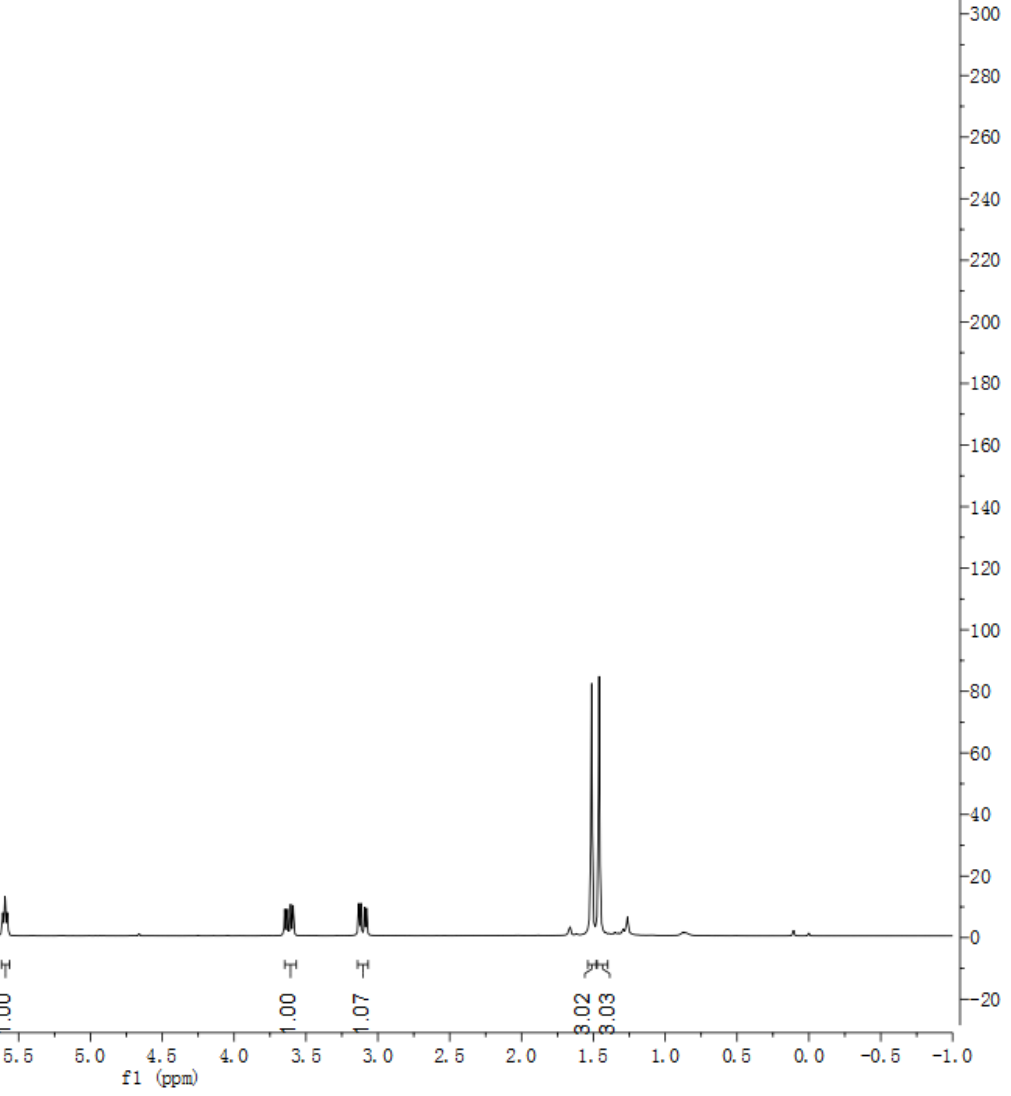

${ }^{13} \mathbf{C}$ NMR $\left(100 \mathrm{MHz}, \mathrm{CDCl}_{3}\right)$<smiles>CC(C)(OOC(CC(=O)c1ccccc1)c1ccccc1)c1ccccc1</smiles>

(S)-1s
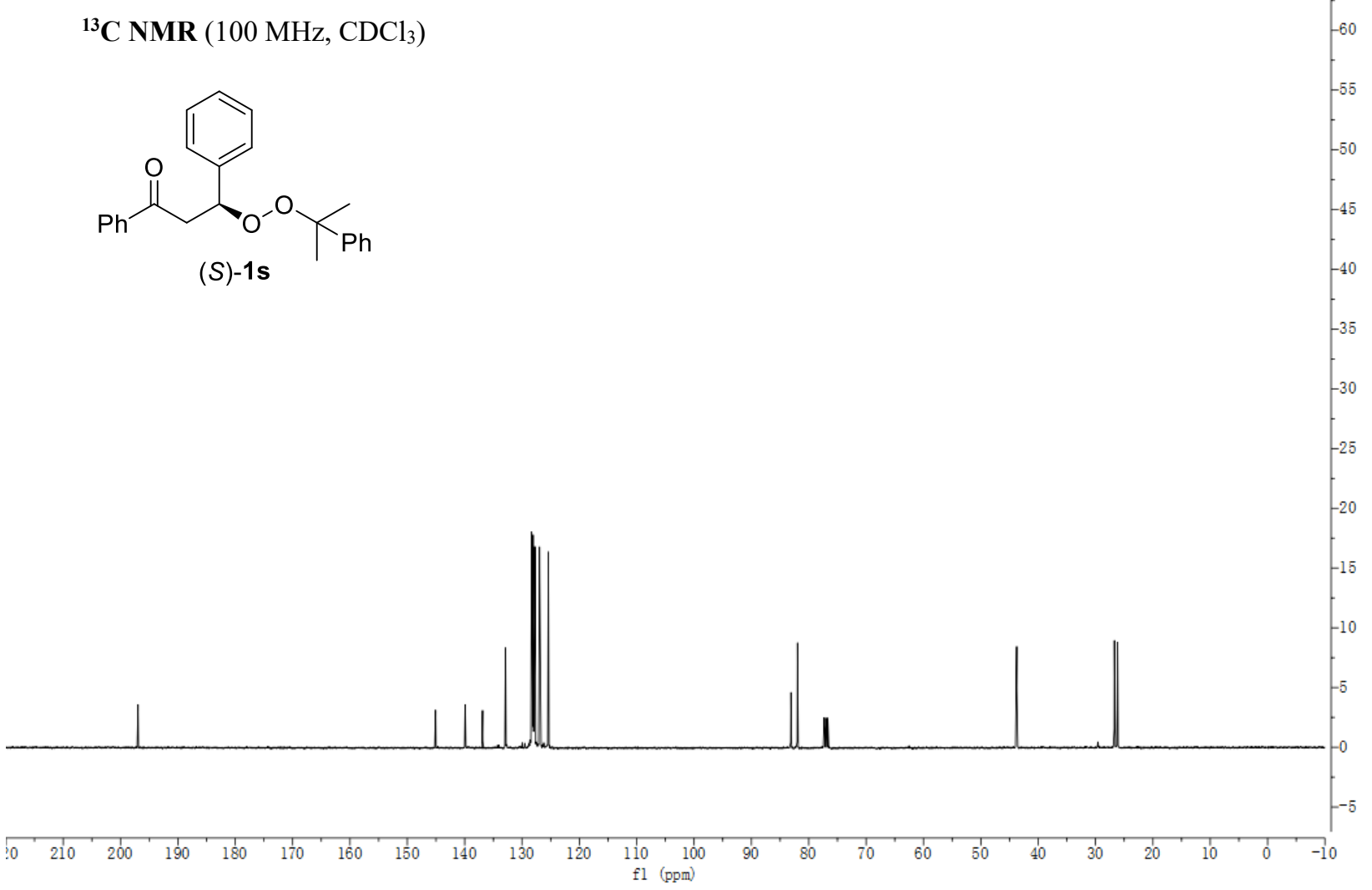
${ }^{1} \mathbf{H}$ NMR (400 MHz, $\mathrm{CDCl}_{3}$ )<smiles>O=C(c1ccccc1)[C@H]1O[C@H]1c1ccccc1</smiles>
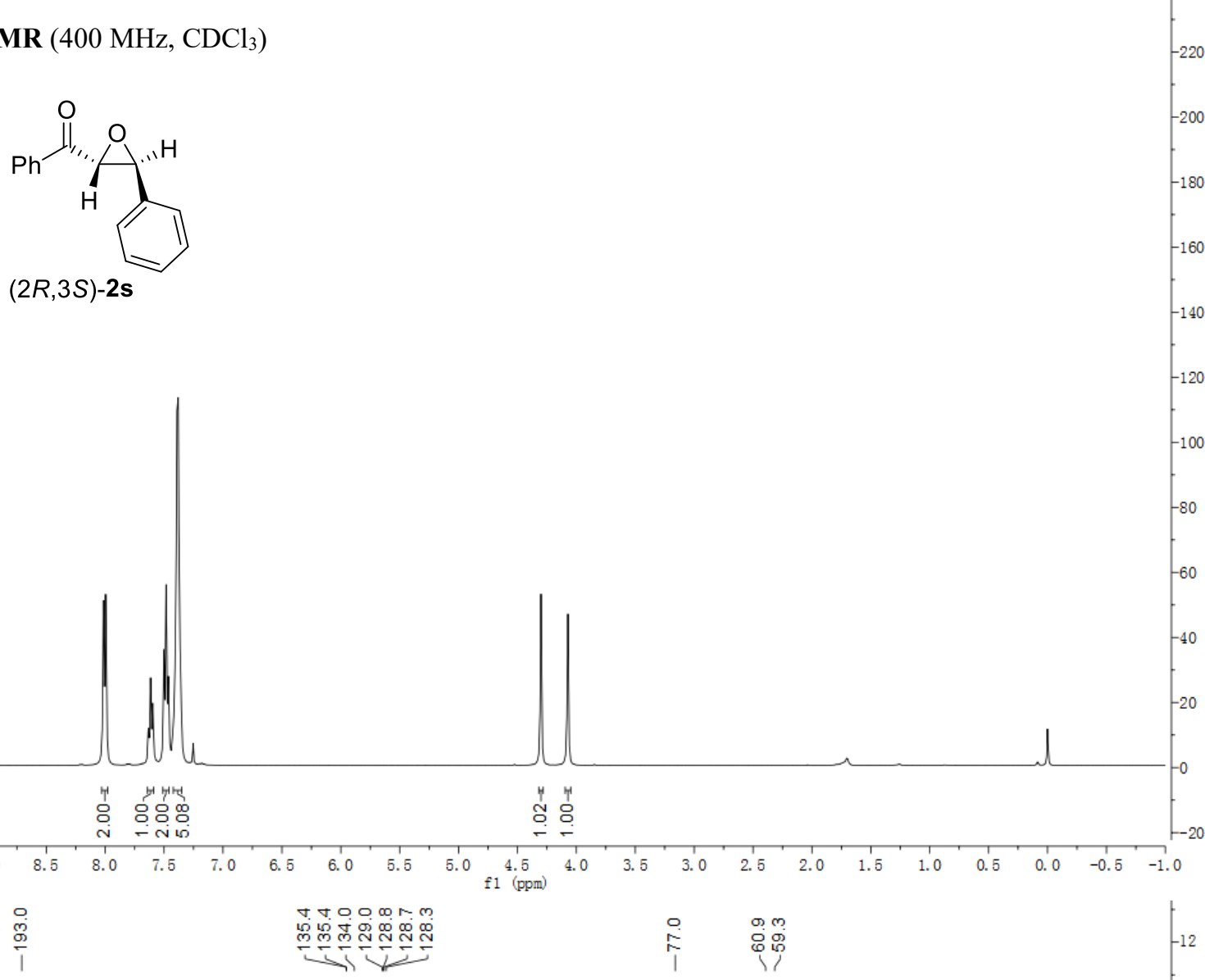

${ }^{13} \mathbf{C ~ N M R}\left(100 \mathrm{MHz}, \mathrm{CDCl}_{3}\right)$<smiles>O=C(c1ccccc1)[C@H]1O[C@H]1c1ccccc1</smiles>

$(2 R, 3 S)-2 \mathbf{s}$

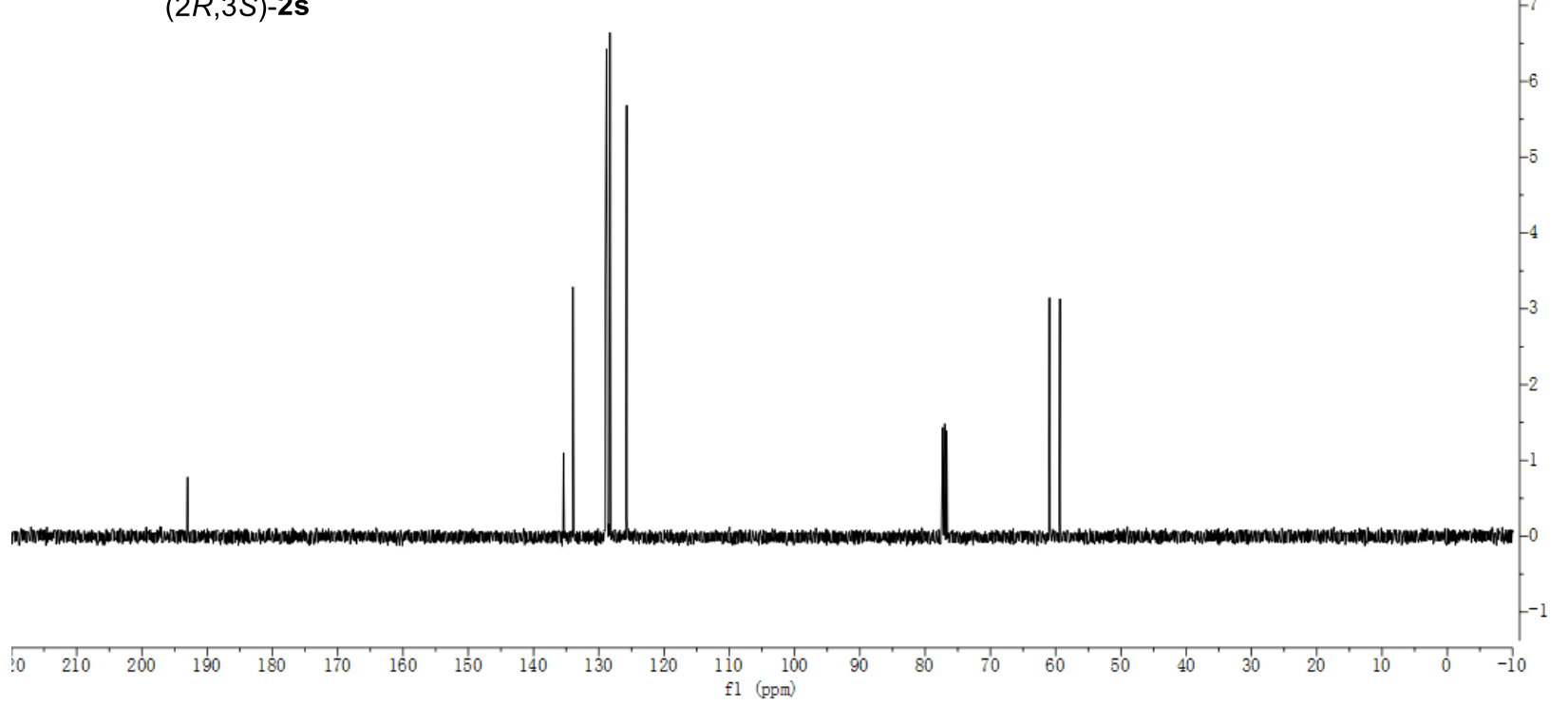


X. ${ }^{1} \mathrm{H}$ and ${ }^{13} \mathrm{C}$ NMR spectra of compounds $(3,4,5,6,7)$

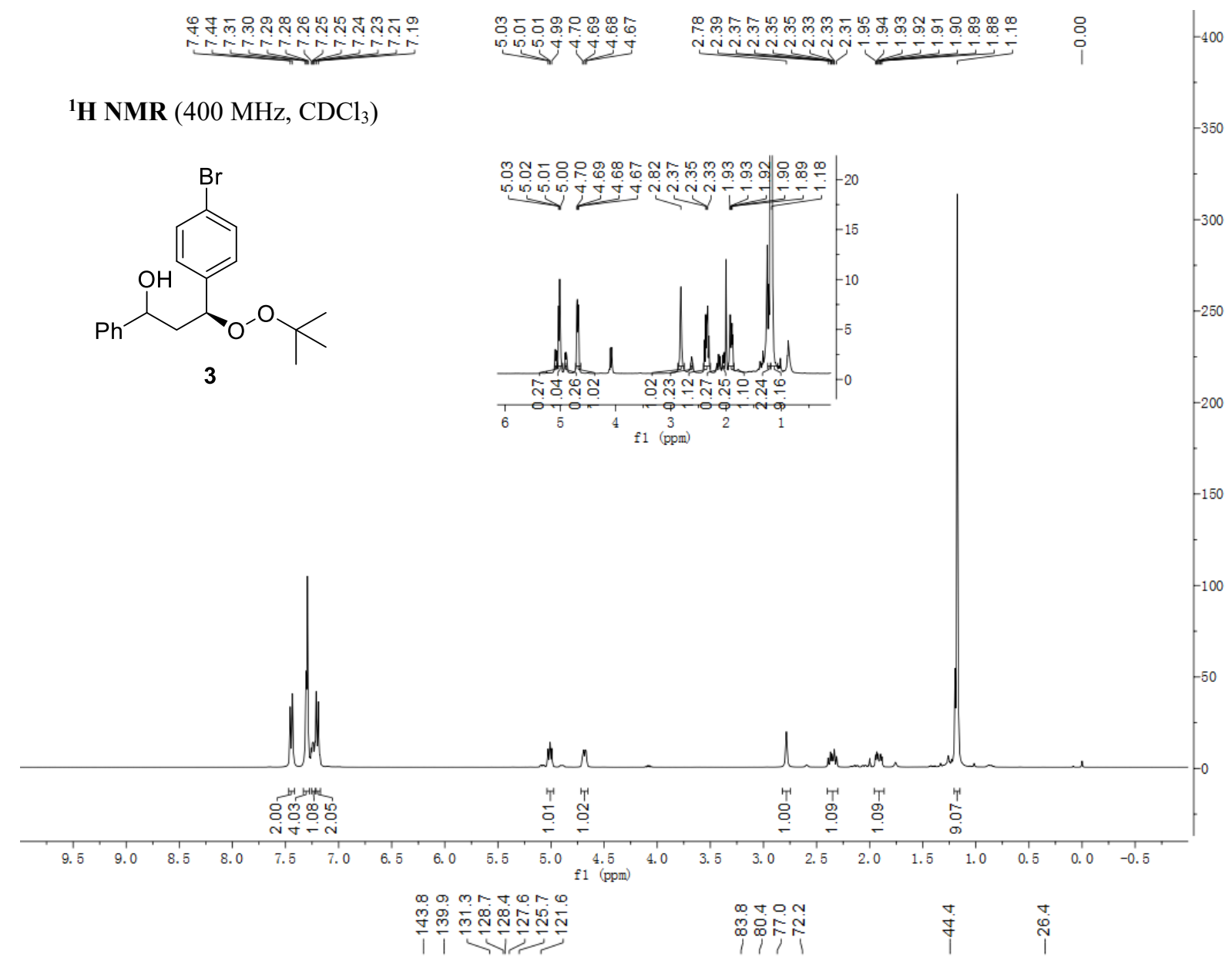

${ }^{13} \mathbf{C}$ NMR (100 MHz, $\left.\mathrm{CDCl}_{3}\right)$<smiles>CC(C)(C)OOC(CC(O)c1ccccc1)c1ccc(Br)cc1</smiles>

3

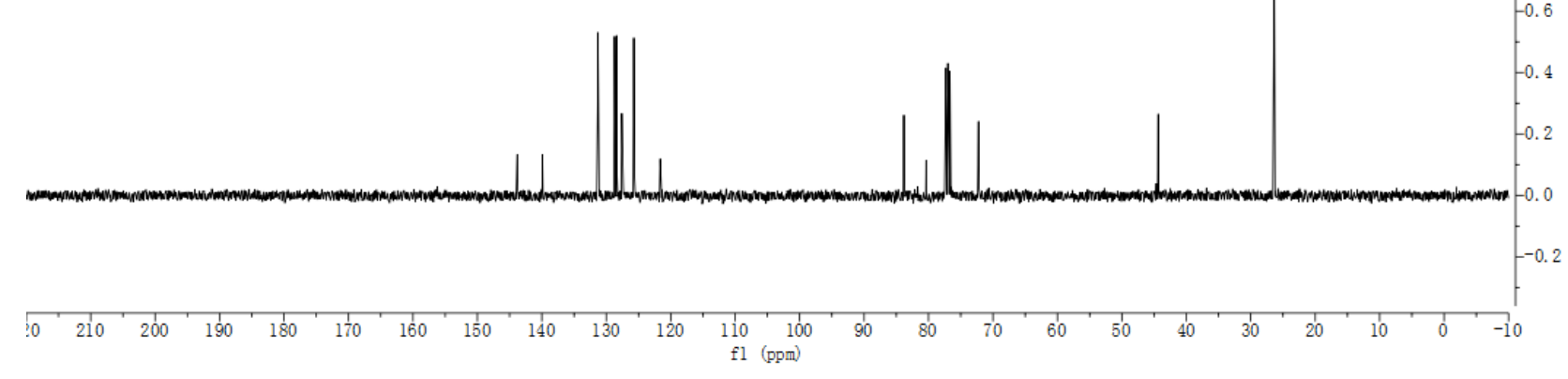


${ }^{1} \mathbf{H}$ NMR $\left(400 \mathrm{MHz}, \mathrm{CDCl}_{3}\right)$<smiles>CC(C)(C)OOC(C/C(=N/Nc1ccc(Br)cc1[N+](=O)[O-])c1ccccc1)c1ccc(Br)cc1</smiles>

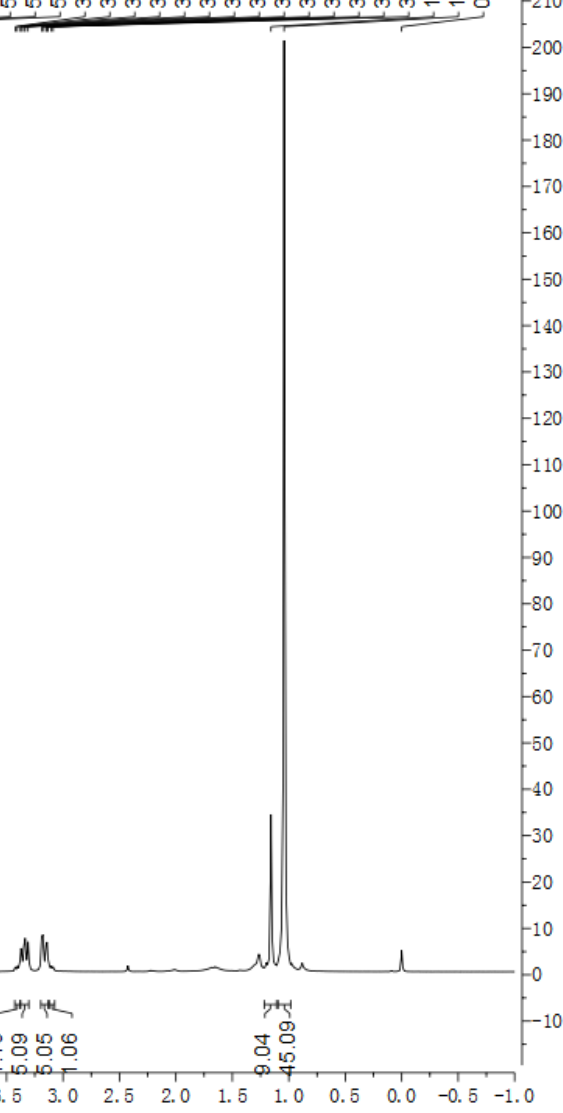

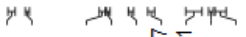

T.

웅

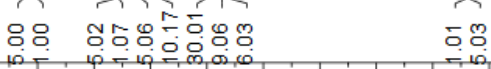

$\stackrel{0}{\circ}$

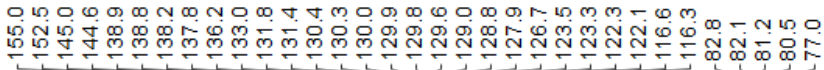

${ }^{13} \mathbf{C}$ NMR (100 MHz, $\left.\mathrm{CDCl}_{3}\right)$

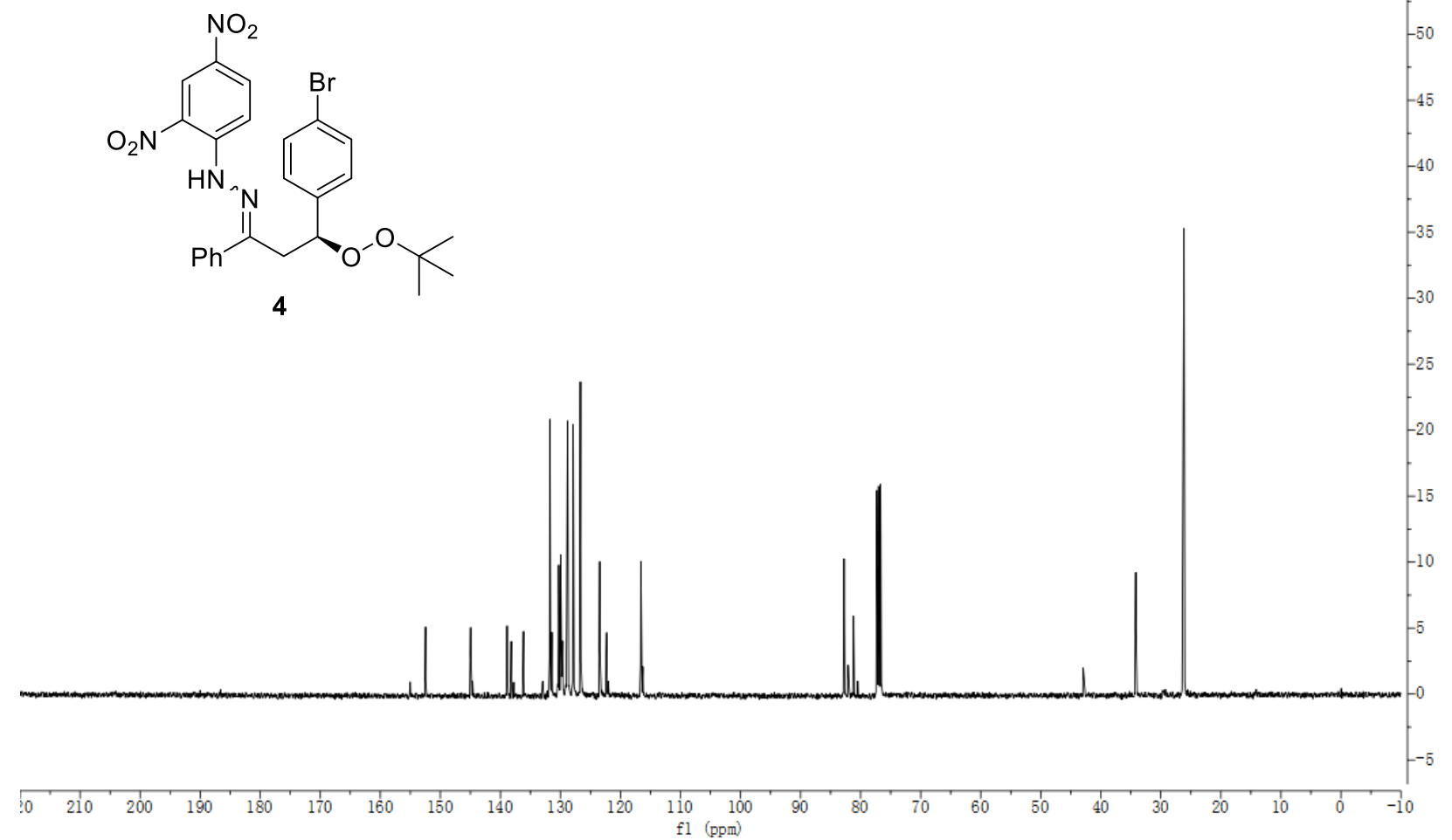


${ }^{1} \mathbf{H}$ NMR $\left(400 \mathrm{MHz}, \mathrm{CDCl}_{3}\right)$<smiles>CC(C)(C)COC(=O)C(C/C(=N/Nc1ccc([N+](=O)[O-])cc1[N+](=O)[O-])c1ccccc1)OC(C)(C)C</smiles>

4a

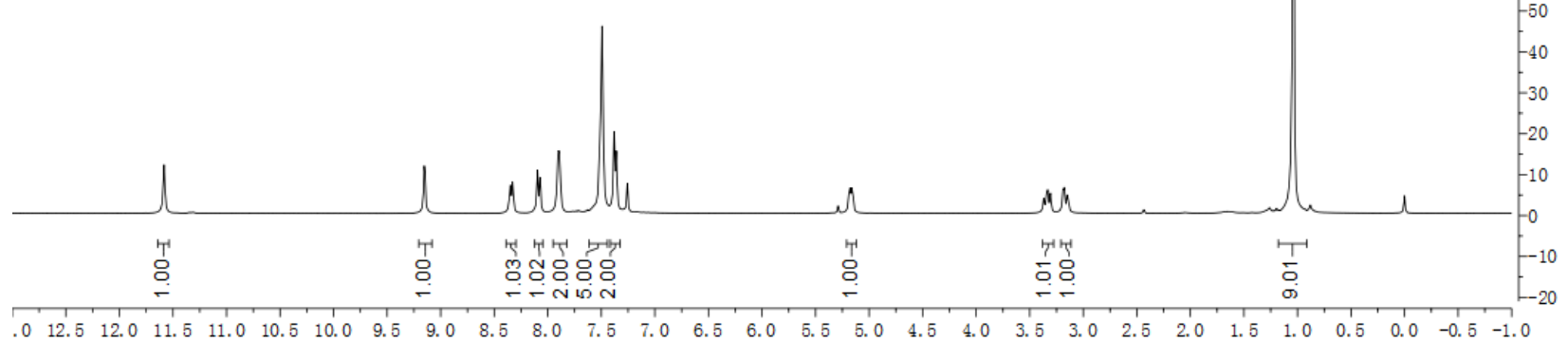
$\begin{array}{lllllllllllllllllllllllllllllllll}.0 & 12.5 & 12.0 & 11.5 & 11.0 & 10.5 & 10.0 & 9.5 & 9.0 & 8.5 & 8.0 & 7.5 & 7.0 & 6.5 & 6.0 & 5.5 & 5.0 & 4.5 & 4.0 & 3.5 & 3.0 & 2.5 & 2.0 & 1.5 & 1.0 & 0.5 & 0.0 & -0.5 & -1.0\end{array}$

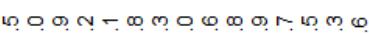

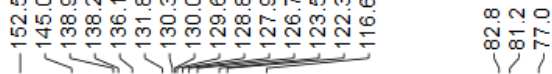

${ }^{13} \mathbf{C}$ NMR $\left(100 \mathrm{MHz}, \mathrm{CDCl}_{3}\right)$<smiles>CCC(Br)/C=C\C(C/C(=N/Nc1ccc([N+](=O)[O-])cc1[N+](=O)[O-])c1ccccc1)(OOC(C)(C)C)c1ccccc1</smiles>

$4 a$

\section{ले ब্ं}

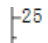

-25
-24 -230
-220 


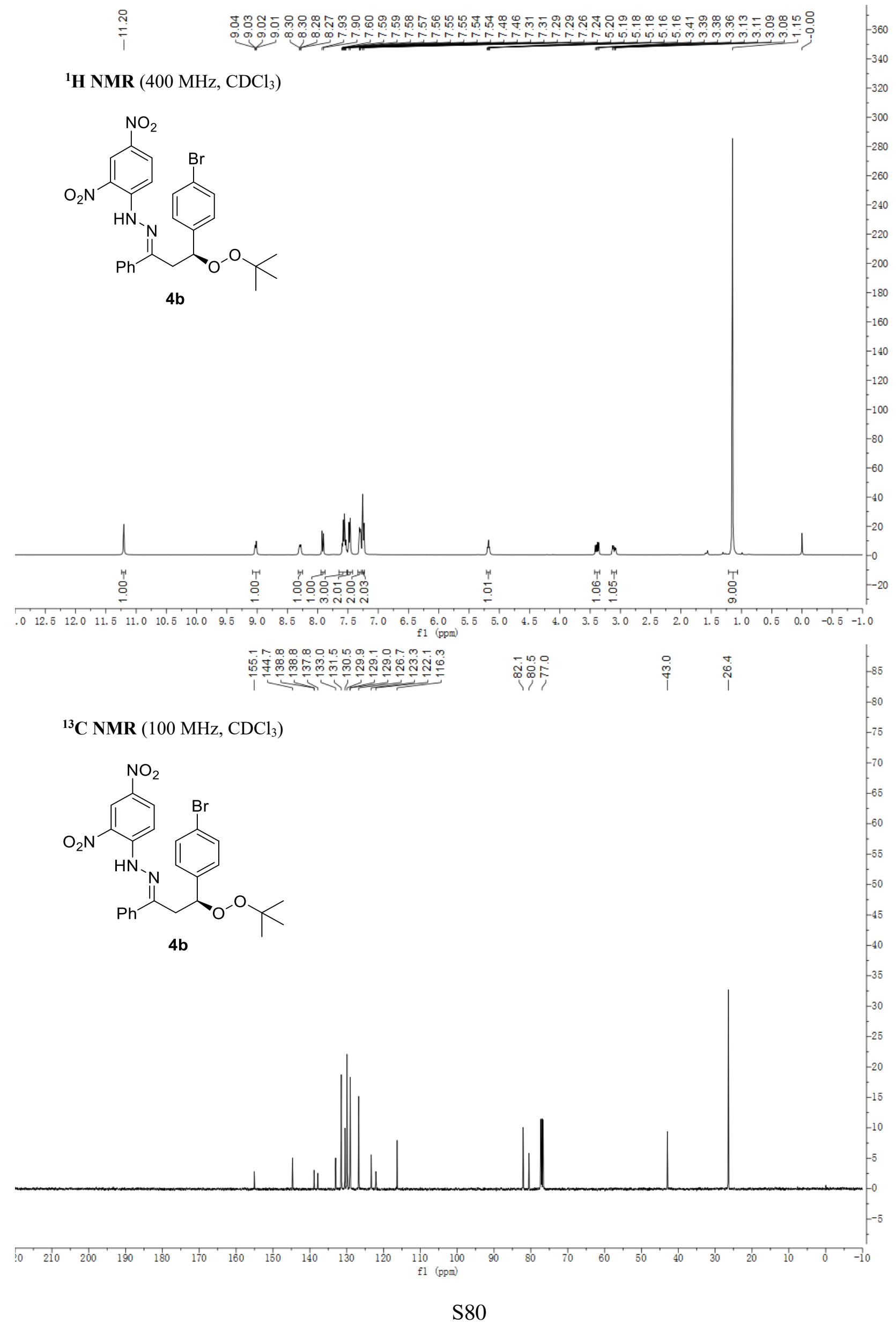




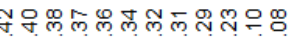
miñ

${ }^{1} \mathbf{H}$ NMR $\left(400 \mathrm{MHz}, \mathrm{CDCl}_{3}\right)$<smiles>OC(c1ccccc1)[C@H]1O[C@H]1c1ccc(Br)cc1</smiles>

प

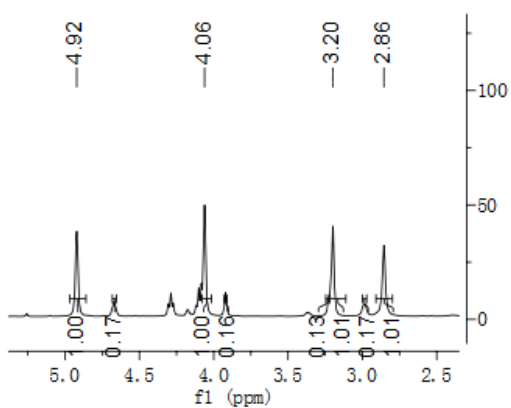

i̊

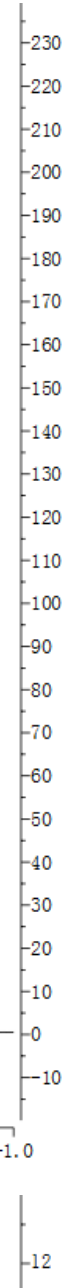

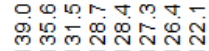

$---\frac{1}{-1}$

${ }^{13}$ C NMR $\left(100 \mathrm{MHz}, \mathrm{CDCl}_{3}\right)$<smiles>OC(c1ccccc1)[C@H]1O[C@H]1c1ccc(Br)cc1</smiles>

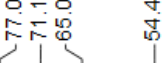 \\ 离}
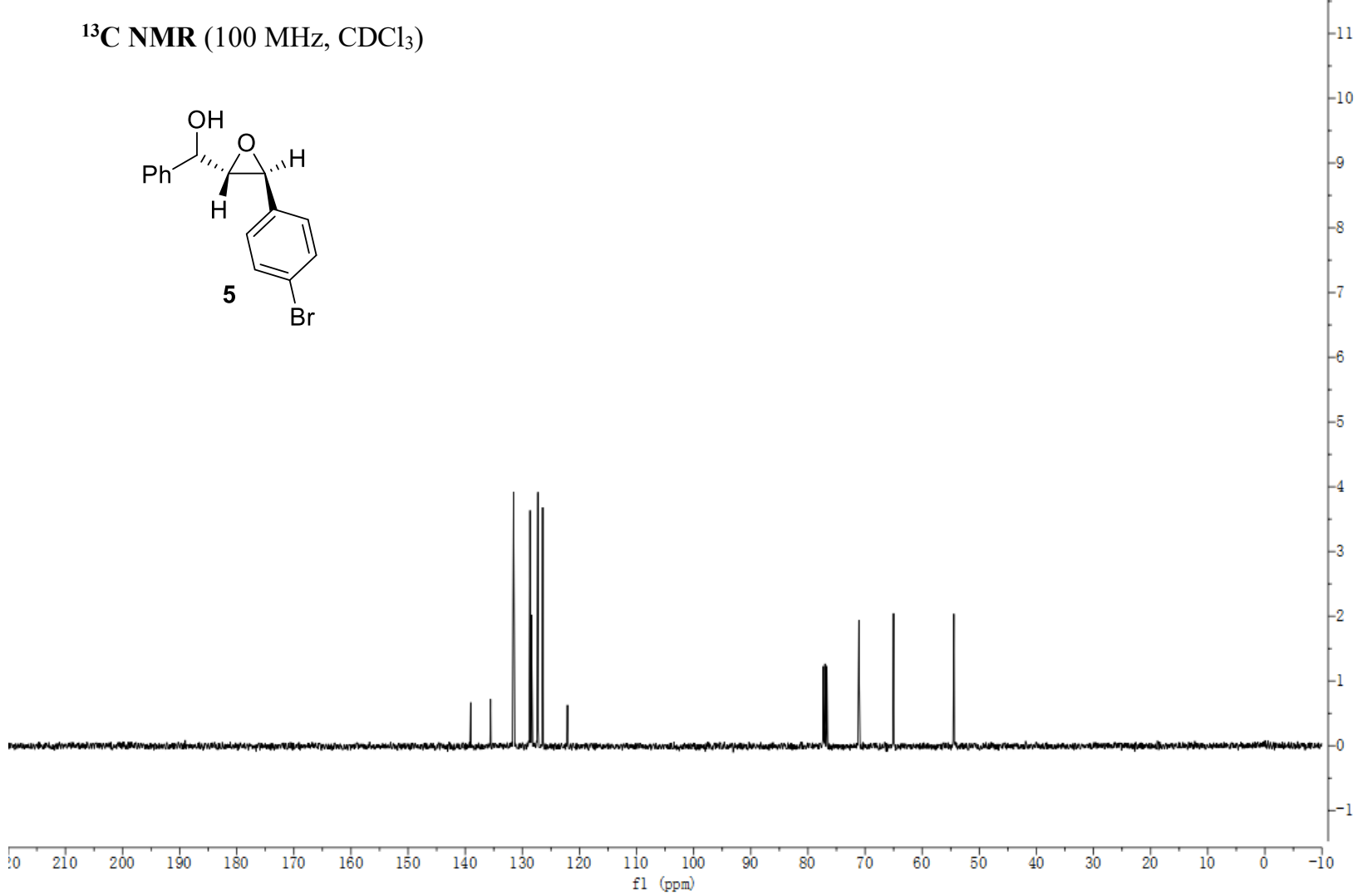
궁ำ

लु

$\stackrel{\leftrightarrow}{i}$

${ }^{1} \mathbf{H}$ NMR (400 MHz, $\left.\mathrm{CDCl}_{3}\right)$<smiles>O=C(c1ccccc1)[C@H](O)[C@H](Cl)c1ccc(Br)cc1</smiles>

6
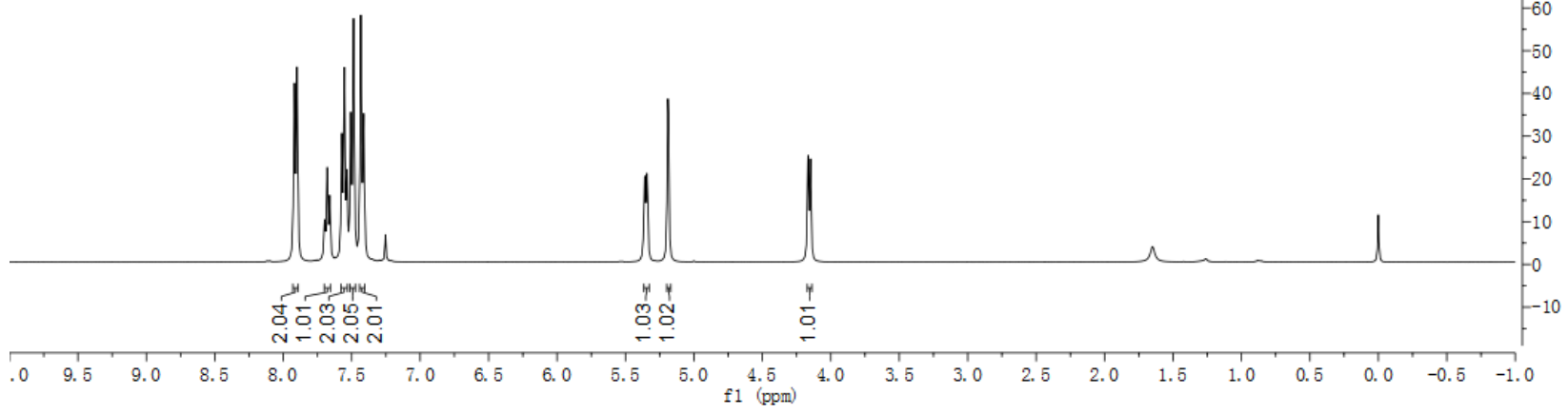

$\stackrel{m}{\stackrel{m}{*}}$

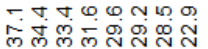

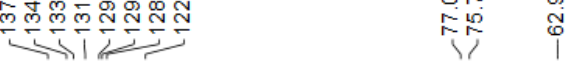

${ }^{13}$ C NMR (100 MHz, $\left.\mathrm{CDCl}_{3}\right)$<smiles>O=C(c1ccccc1)[C@H](O)[C@H](Cl)c1ccc(Br)cc1</smiles>

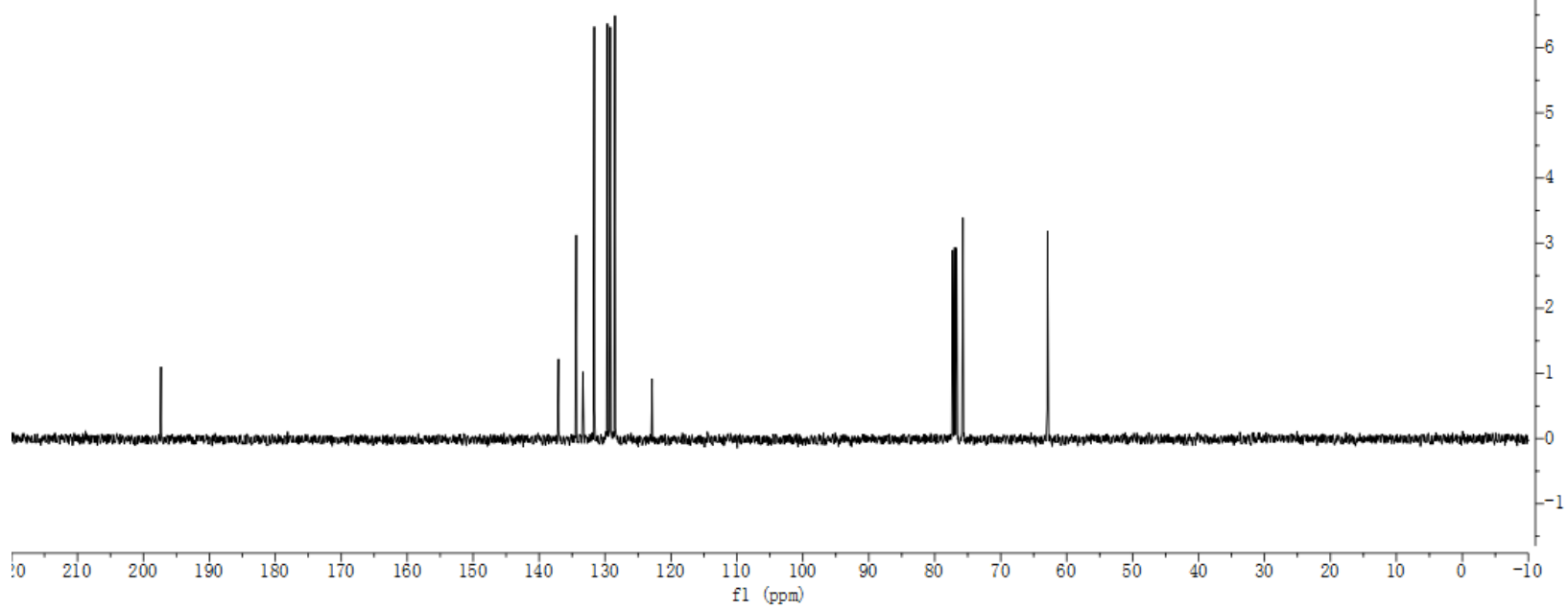




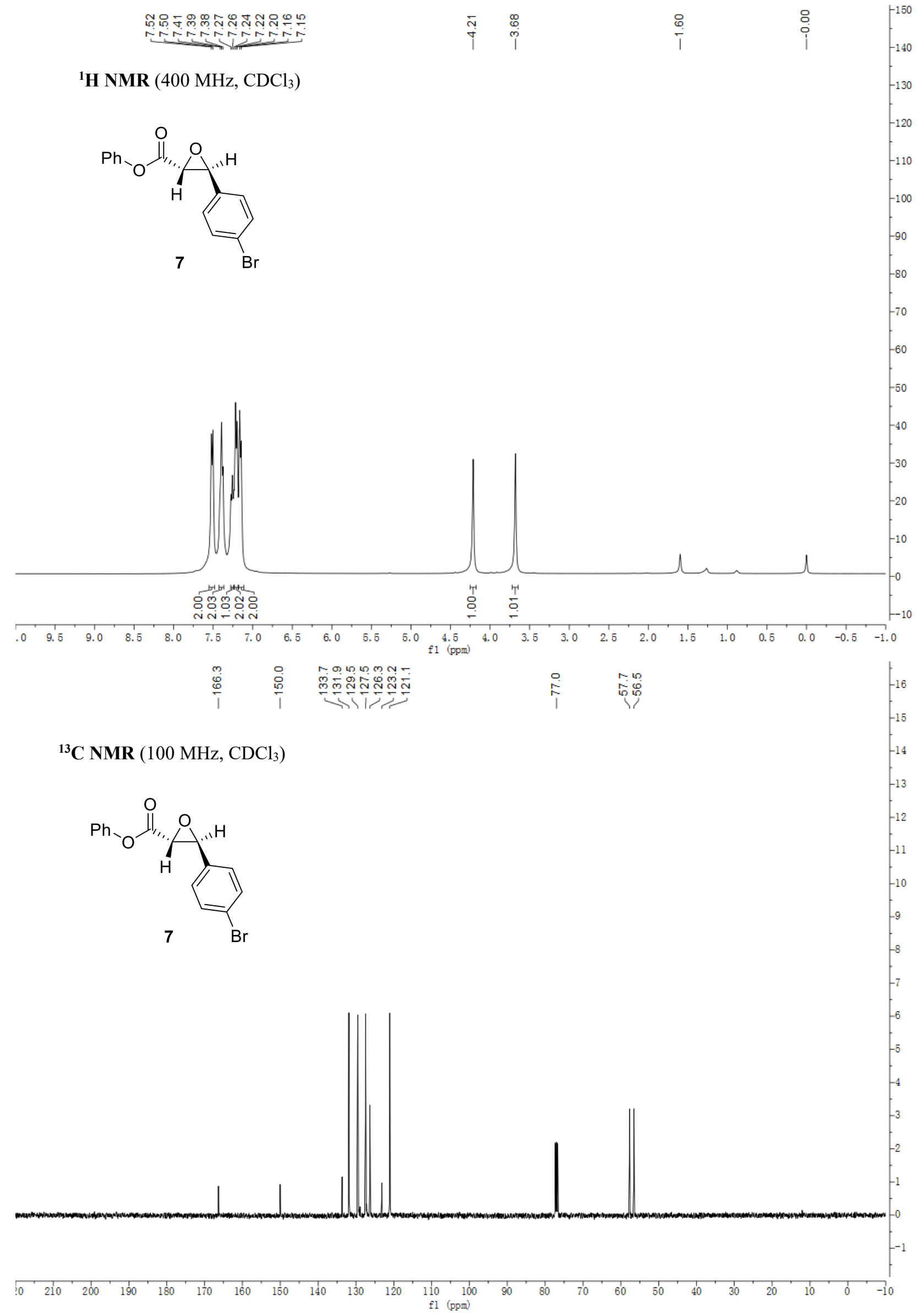


XI. X-ray crystallographic information (1p, 2p, 7)<smiles>CC(C)(C)OO[C@H](CC(=O)c1ccccc1)c1ccc(Br)cc1</smiles>

$(S)-1 p$

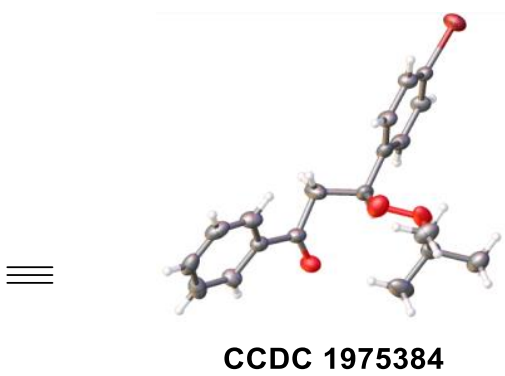

Wavelength $=0.71073$

$\mathrm{c}=28.8797(9)$

gamma $=90$

Temperature:

$150 \mathrm{~K}$

Calculated

Reported

Volume

$1767.79(13)$

$1767.79(13)$

Space group

P 212121

P 212121

Hall group

$\mathrm{P} 2 \mathrm{ac} 2 \mathrm{ab}$

$\mathrm{P} 2 \mathrm{ac} 2 \mathrm{ab}$

Moiety formula

$\mathrm{C} 19 \mathrm{H} 21 \mathrm{Br} \mathrm{O} 3$

$\mathrm{C} 19 \mathrm{H} 21 \mathrm{Br} \mathrm{O} 3$

Sum formula

$\mathrm{C} 19 \mathrm{H} 21 \mathrm{BrO} 3$

C19 H21 Br O3

377.26

377.27

Dx,g cm-3

Z

1.418

1.418

4

4

$\mathrm{Mu}(\mathrm{mm}-1)$

2.337

2.337

F000

776.0

776.0

775.18

7, 14, 39

7, 14,36

4612 [2676]

3987

$0.576,0.657$

$0.709,1.000$

Tmin'

0.515

Correction method $=\#$ Reported T Limits: Tmin $=0.709$ Tmax $=1.000$

AbsCorr $=$ MULTI-SCAN

Data completeness $=1.49 / 0.86$

$\mathrm{R}($ reflections $)=0.0521(3234)$

$\mathrm{S}=1.041$
Theta $(\max )=28.803$

wR2 (reflections) $=0.1243(3987)$

Npar $=211$

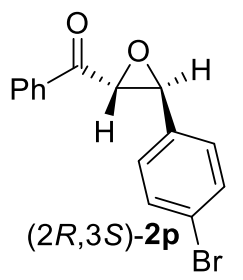

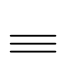

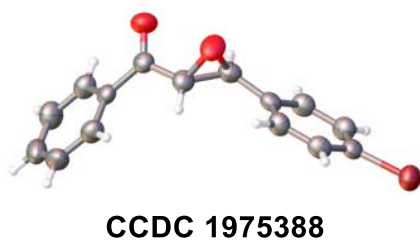




\begin{tabular}{|c|c|c|c|}
\hline Bond precision: & $\mathrm{C}-\mathrm{C}=0.0058 \mathrm{~A}$ & & Wavelength $=0.71073$ \\
\hline \multirow[t]{2}{*}{ Cell: } & $\mathrm{a}=5.8578(2)$ & $\mathrm{b}=11.5548(6)$ & $\mathrm{c}=18.4277(8)$ \\
\hline & alpha $=90$ & beta $=90$ & gamma $=90$ \\
\hline \multirow[t]{2}{*}{ Temperature: } & $295 \mathrm{~K}$ & & \\
\hline & Calculated & Reported & \\
\hline Volume & $1247.29(9)$ & $1247.29(9)$ & \\
\hline Space group & P 212121 & P 212121 & \\
\hline Hall group & P $2 a c 2 a b$ & P $2 a c 2 a b$ & \\
\hline Moiety formula & $\mathrm{C} 15 \mathrm{H} 11 \mathrm{Br} \mathrm{O} 2$ & $\mathrm{C} 15 \mathrm{H} 11 \mathrm{Br} \mathrm{O} 2$ & \\
\hline Sum formula & C15 H11 Br O2 & C15 H11 Br O2 & \\
\hline $\mathrm{Mr}$ & 303.14 & 303.15 & \\
\hline Dx,g cm-3 & 1.614 & 1.614 & \\
\hline $\mathrm{Z}$ & 4 & 4 & \\
\hline $\mathrm{Mu}(\mathrm{mm}-1)$ & 3.285 & 3.285 & \\
\hline F000 & 608.0 & 608.0 & \\
\hline F000' & 607.12 & & \\
\hline $\mathrm{h}, \mathrm{k}, \operatorname{lmax}$ & $7,15,24$ & $7,14,24$ & \\
\hline Nref & $3248[1891]$ & 2831 & \\
\hline Tmin,Tmax & $0.345,0.412$ & $0.776,1.000$ & \\
\hline Tmin' & 0.219 & & \\
\hline
\end{tabular}

Correction method $=\#$ Reported T Limits: Tmin $=0.776 \mathrm{Tmax}=1.000$

AbsCorr $=$ MULTI-SCAN

Data completeness $=1.50 / 0.87$

Theta $(\max )=28.764$

$\mathrm{R}$ (reflections) $=0.0389(2102)$

wR2 (reflections) $=0.0617(2831)$

$\mathrm{S}=1.021$

Npar $=163$
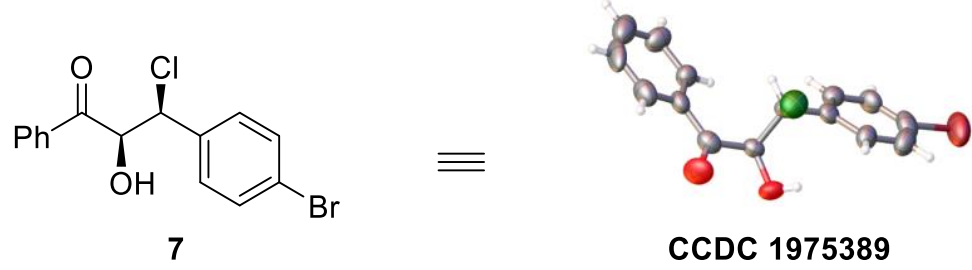

$\begin{array}{llll}\text { Bond precision: } & \mathrm{C}-\mathrm{C}=0.0146 \mathrm{~A} & & \text { Wavelength }=0.71073 \\ \text { Cell: } & \mathrm{a}=7.5868(9) & \mathrm{b}=8.4128(11) & \mathrm{c}=11.4484(15) \\ & \text { alpha }=90 & \text { beta }=103.183(13) & \text { gamma }=90 \\ \text { Temperature: } & 295 \mathrm{~K} & & \\ & \text { Calculated } & \text { Reported } & \\ \text { Volume } & 711.45(16) & 711.45(16) & \\ \text { Space group } & \text { P 21 } & \text { P } 1211 & \\ \text { Hall group } & \text { P 2yb } & \text { P 2yb } & \end{array}$ 


$\begin{array}{lll}\text { Moiety formula } & \mathrm{C} 15 \mathrm{H} 12 \mathrm{Br} \mathrm{Cl} \mathrm{O} 2 & \mathrm{C} 15 \mathrm{H} 12 \mathrm{Br} \mathrm{Cl} \mathrm{O} 2 \\ \text { Sum formula } & \mathrm{C} 15 \mathrm{H} 12 \mathrm{Br} \mathrm{Cl} \mathrm{O} 2 & \mathrm{C} 15 \mathrm{H} 12 \mathrm{Br} \mathrm{Cl} \mathrm{O} 2 \\ \mathrm{Mr} & 339.60 & 339.61 \\ \mathrm{Dx}, \mathrm{g} \mathrm{cm}-3 & 1.585 & 1.585 \\ \mathrm{Z} & 2 & 2 \\ \mathrm{Mu}(\mathrm{mm}-1) & 3.071 & 3.071 \\ \text { F000 } & 340.0 & 340.0 \\ \text { F000' } & 339.86 & \\ \text { h,k,lmax } & 10,11,15 & 10,11,14 \\ \text { Nref } & 3759[2003] & 3219 \\ \text { Tmin, Tmax } & 0.377,0.423 & 0.420,1.000 \\ \text { Tmin' } & 0.349 & \end{array}$

Correction method $=\#$ Reported T Limits: Tmin $=0.420$ Tmax $=1.000$

AbsCorr $=$ MULTI-SCAN

Data completeness $=1.61 / 0.86$

Theta $(\max )=28.886$

$\mathrm{R}$ (reflections) $=0.0626(1894)$

wR2 (reflections) $=0.1595(3219)$

$\mathrm{S}=1.033$

Npar $=173$

\section{References}

[1] Y. Yao, Z. Wang, B. Wang, Org. Chem. Front. 2018, 5, 2501-2504.

[2] S. Luo, N. Zhang, Z. Wang, H. Yan, Org. Biomol. Chem. 2018, 16, 2893-2901.

[3] W. Liu, Y. Li, K. Liu, Z. Li, J. Am. Chem. Soc. 2011, 133, 10756-10759.

[4] N. Ahmed, G. K. Pathe, S. Jheeta, RSC Adv. 2015, 5, 63095-63103.

[5] M. Waheed, N. Ahmed, Synth. 2017, 49, 4372-4382.

[6] E. J. Corey, F. Y. Zhang, Org. Lett. 1999, 1, 1287-1290.

[7] S. Huber, M. Cokoja, M. Drees, J. Mínk, F. E. Kühn, Catal. Sci. Technol. 2013, 3, 388-393. 